\title{
Investigation of the Impacts of Thermal Activated Sludge Pretreatment and Development of a Pretreatment Model
}

\author{
by \\ Gillian Staples-Burger \\ A thesis \\ presented to the University of Waterloo \\ in fulfillment of the \\ thesis requirement for the degree of \\ Master of Applied Science \\ in \\ Civil Engineering
}

Waterloo, Ontario, Canada, 2012

(C) Gillian Staples-Burger 2012 


\section{Author's Declaration}

I hereby declare that I am the sole author of this thesis. This is a true copy of the thesis, including any required final revisions, as accepted by my examiners. I understand that my thesis may be made electronically available to the public. 


\begin{abstract}
Waste activated sludge (WAS) pretreatment technologies are typically evaluated in terms of the associated improvement in biogas and sludge production during digestion and post-digestion dewaterability. However, WAS properties, and hence the impact of pretreatment on WAS properties, are dependent upon the raw wastewater composition and configuration of the wastewater treatment plant (WWTP). A generally accepted means of characterizing and comparing all pretreatment processes does not exist. The motivation for this project was to evaluate the impact of pretreatment on WAS properties in terms of changes in COD fractionation. The first objective of this study was to fractionate the COD of the WAS before and after pretreatment to show how pretreatment may increase the rate and extent of aerobic digestion. The second objective was to develop a COD-based stoichiometric pretreatment model that may be integrated into WWTP simulations.
\end{abstract}

A bench-scale biological reactor (BR) with a solids retention time (SRT) of 5 days was started up with WAS from the Waterloo WWTP. The BR was fed daily with a completely biodegradable synthetic substrate so that the BR WAS contained only biomass and decay products after 3 SRTs of operation. In the first phase of the study, an aerobic digester (AD) with a SRT of $10 \mathrm{~d}$ was fed daily with BR WAS. The BR-AD system was operated at steady state for one month. A range of physical and biochemical properties were regularly measured in each process stream. Offline respirometric tests were regularly conducted to determine the aerobic degradability and fractionate the COD of the BR and AD WAS. The oxygen uptake rate (OUR) associated with the daily addition of BR WAS to the $\mathrm{AD}$ was determined as an additional measurement of the aerobic degradability of the BR WAS.

In the second phase of the study, the BR WAS was pretreated prior to being fed daily to the AD. High pressure thermal hydrolysis (HPTH) pretreatment was selected for this project since it is one of the most popular and promising pretreatment techniques. A sealed volume of BR WAS was heated to $150^{\circ} \mathrm{C}$ at 3 bars for 30 minutes. The same physical, biochemical and biological tests used to characterize the process streams in Phase 1 were employed to characterize those in Phase 2. The Phase 2 system was operated for two months at steady-state.

The results of several independent tests showed that the COD of the BR WAS was comprised of storage products $\left(\mathrm{X}_{\mathrm{STO}}\right)$ in addition to active heterotrophs $\left(\mathrm{Z}_{\mathrm{bh}}\right)$ and decay products $\left(\mathrm{Z}_{\mathrm{e}}\right)$. However, it was shown that the AD WAS only contained $Z_{b h}$ and $Z_{e}$ as $X_{\text {STO }}$ was depleted in the AD. 
HPTH pretreatment did not reduce the TCOD concentration of the WAS however it did solubilize 56 $\pm 7 \%$ of COD, $49 \% \pm 11 \%$ of organic nitrogen, $56 \pm 10 \%$ of VSS and did not solubilize ISS. Furthermore, pretreatment did not generate soluble non-biodegradable COD. These findings were consistent with prior research on HPTH WAS pretreatment.

Pretreatment increased the rate at which the BR WAS was aerobically degraded. The offline respirometric tests showed that the pretreated BR WAS contained a substantial amount of readily biodegradable $\operatorname{COD}\left(\mathrm{S}_{\mathrm{bsc}}\right)$. However, pretreatment did not increase the extent of biodegradation. The results of several independent tests showed that the non-biodegradable COD component of the BR WAS, i.e. $Z_{\mathrm{e}}$, was not converted to biodegradable COD by pretreatment.

A COD-based stoichiometric pretreatment model was developed for the dose of HPTH pretreatment employed in this study. When this model was integrated into BioWin ${ }^{\circledR}$, it was able to accurately simulate both the steady state performance of the overall system employed in this study as well as dynamic respirometry results. The experimental results showed that the TCOD of the BR WAS consisted of $51 \% \mathrm{Z}_{\mathrm{bh}}, 12 \% \mathrm{Z}_{\mathrm{e}}$ and $37 \% \mathrm{X}_{\text {Sто }}$ and the pretreated BR WAS consisted of $12 \% \mathrm{Z}_{\mathrm{e}}$ and a negligible amount of $\mathrm{Z}_{\mathrm{bh}}$. The pretreatment model verified these fractions and predicted that the pretreated BR WAS also contained $54 \% \mathrm{~S}_{\mathrm{bsc}}$ and $32 \%$ slowly biodegradable COD $\left(\mathrm{X}_{\mathrm{sp}}\right)$. The approach described in this study may be followed to determine the impacts of pretreatment on $Z_{b h}, Z_{e}$ and $\mathrm{X}_{\text {STO }}$ when other doses of HPTH pretreatment and other pretreatment techniques are employed. 


\section{Acknowledgements}

Throughout this project I was fortunate to be supported by several important individuals and institutions. First and foremost, I would like to sincerely thank my supervisor, Dr. Wayne Parker, for the inspiration and unwavering guidance I needed to complete my thesis work. I am very privileged to have spent two years learning about wastewater treatment from this well-respected expert.

I would like to thank my colleagues at EnviroSim Associates for the modeling guidance and generous in-kind contributions they provided. I would like to express my sincere gratitude to Mark Sobon and Mark Merlau for their technical assistance. I am grateful to Peiman Kianmehr for teaching me several experimental techniques and for patiently answering my many questions about WAS pretreatment. I would also like to thank WeiWei Du and Yaohuan Gao for their assistance in the laboratory.

A special thanks goes to Mihail Filippov for helping me with the operation and maintenance of my bench-scale reactors.

I am grateful for the financial support provided by the University of Waterloo, the Natural Sciences and Engineering Research Council of Canada and the Ministry of Training Colleges and University of Ontario.

Lastly, I would like to acknowledge the endless support of my friends and family. Most of all, I would like to thank my husband, Leon Burger, who supported my decision to return to full-time studies and encouraged me every step along the way. 


\section{Table of Contents}

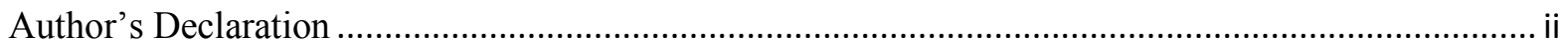

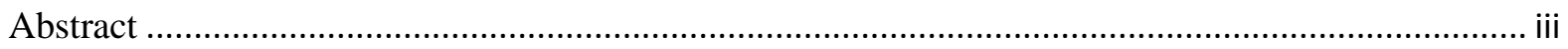

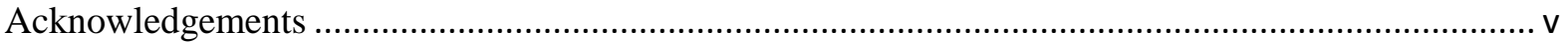

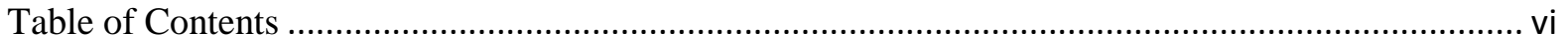

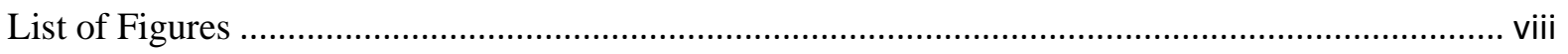

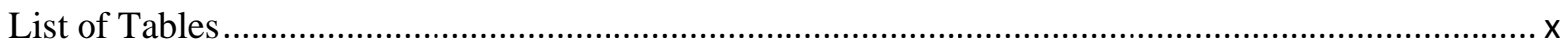

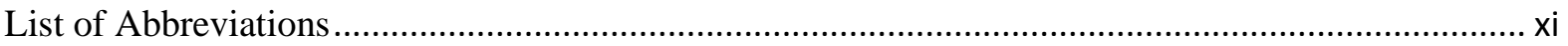

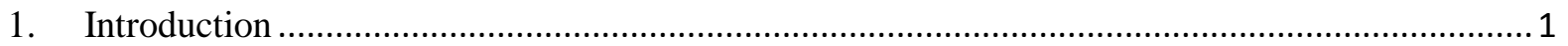

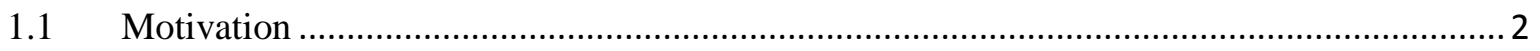

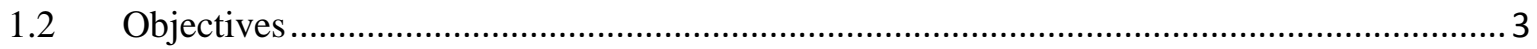

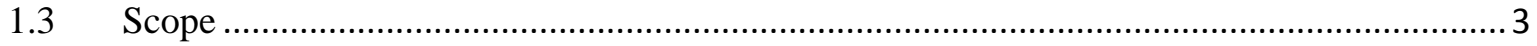

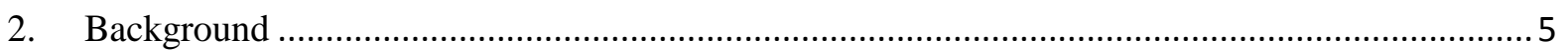

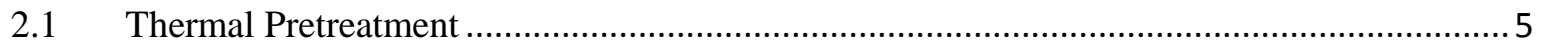

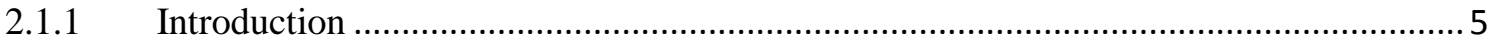

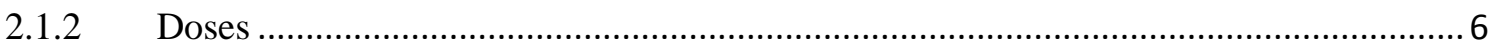

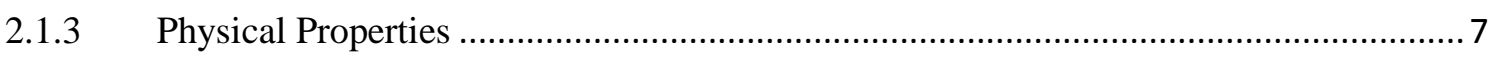

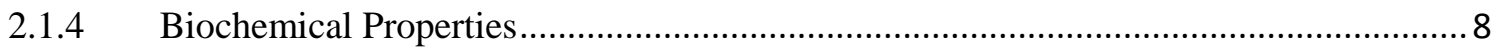

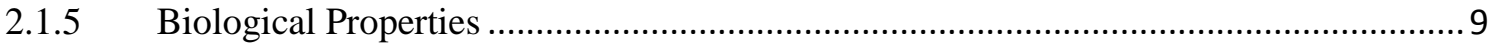

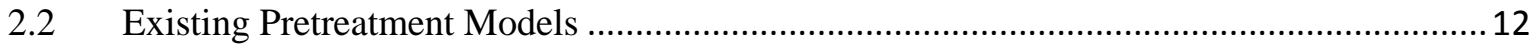

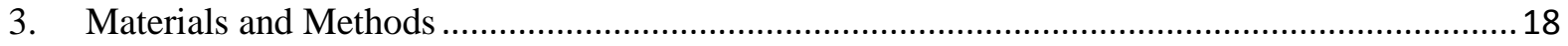

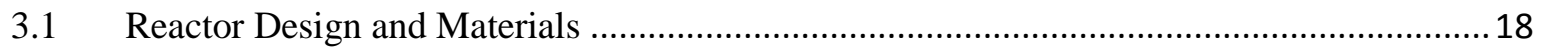

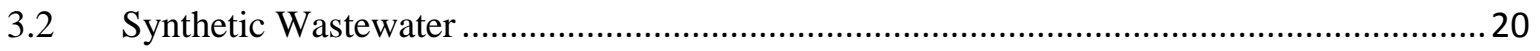

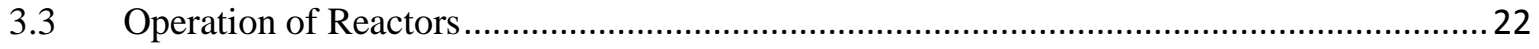

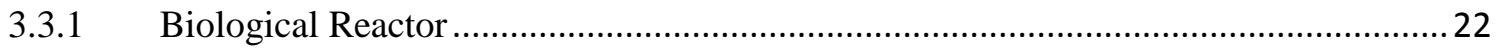

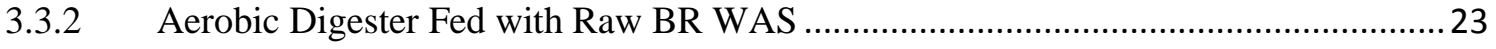

3.3.3 Aerobic Digester Fed with Pretreated BR WAS …................................................... 24

3.4 Project Timeline and Sampling Schedule...................................................................... 26

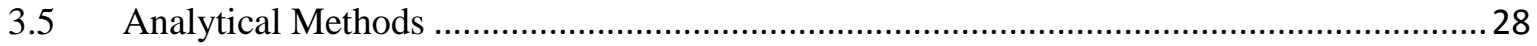

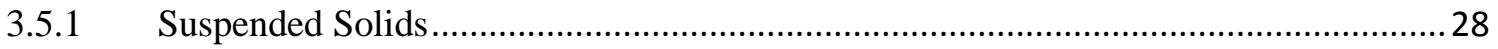

3.5.2 COD

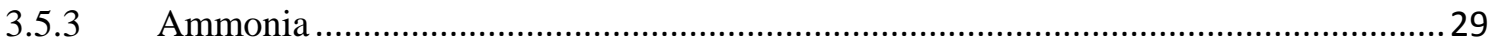




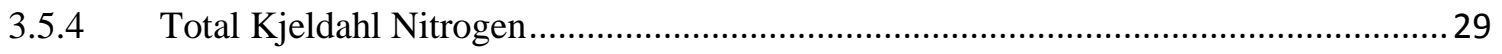

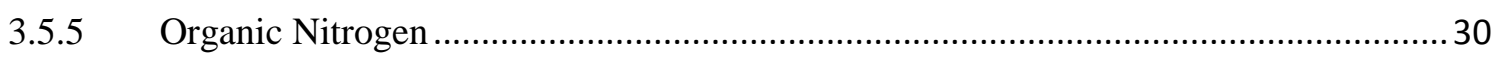

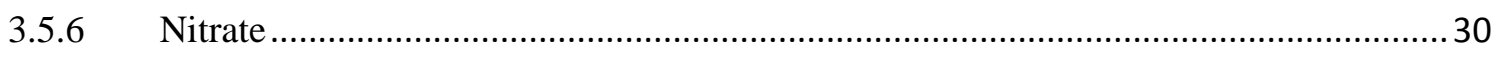

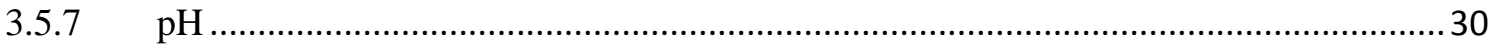

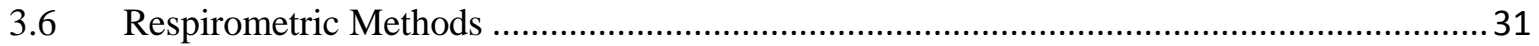

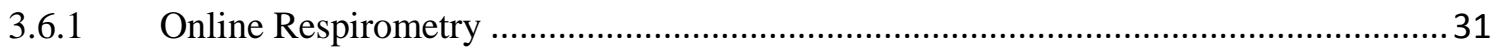

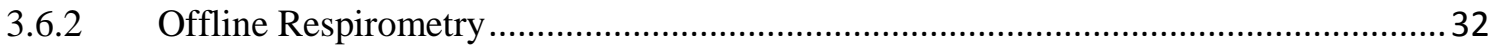

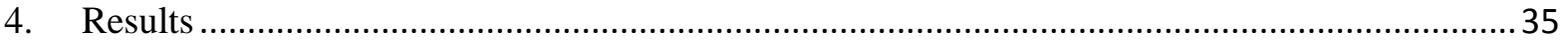

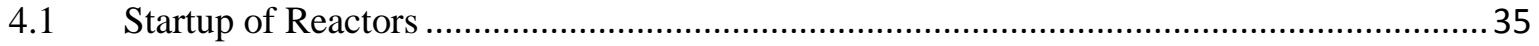

4.2 Physical and Biochemical Characterization of Process Streams ....................................... 38

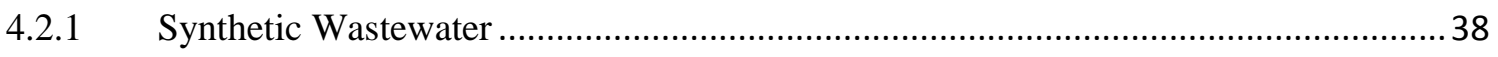

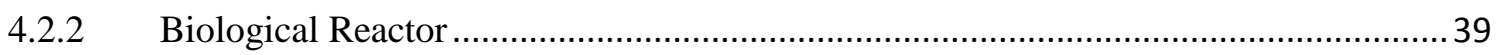

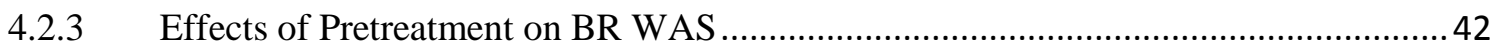

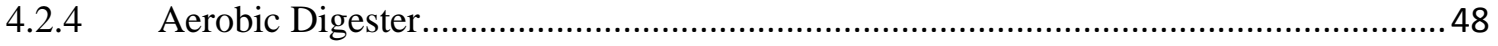

$4.3 \quad$ Biological Analyses of Process Streams …................................................................... 54

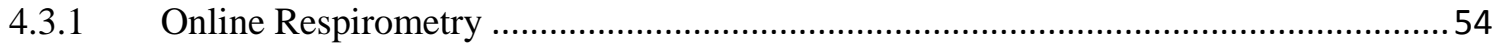

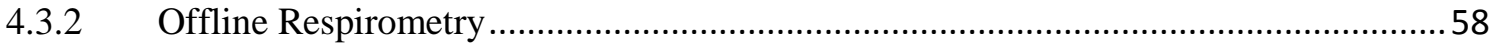

5. Development of the Pretreatment Model ........................................................................... 74

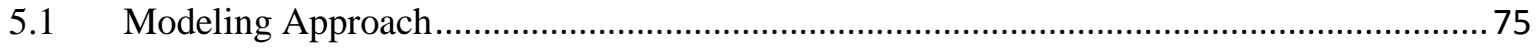

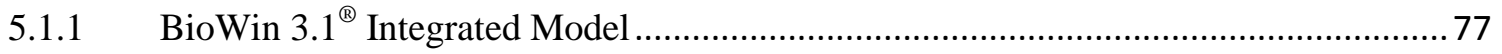

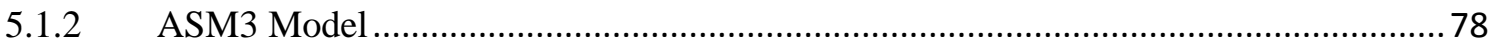

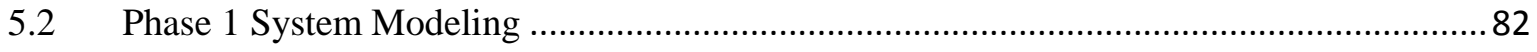

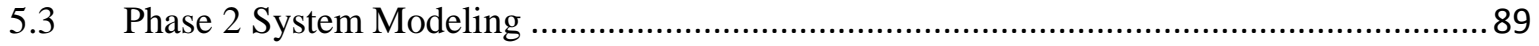

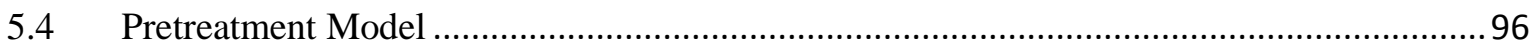

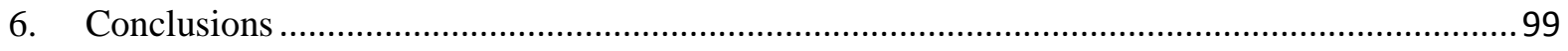

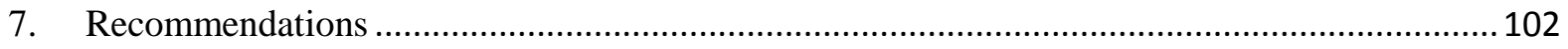

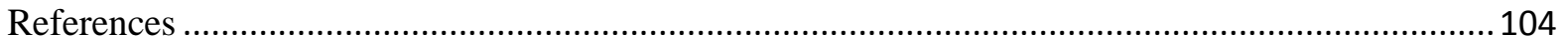

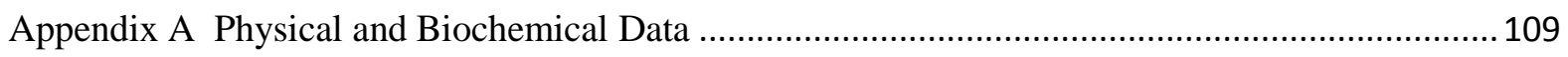

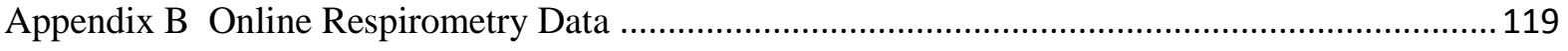

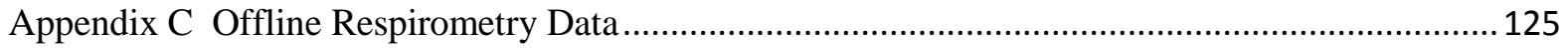




\section{List of Figures}

Figure 2.1 MLSS Composition of Activated Sludge Systems (Ramdani et al., 2010).......................16

Figure 3.1 Phase 1 Process Flow Diagram (Flow in L/d) ........................................................... 18

Figure 3.2 Phase 2 Process Flow Diagram (Flow in L/d) ........................................................... 18

Figure 3.3 Biological Reactor and Aerobic Digester ................................................................. 19

Figure 3.4 Parr® 4563 Mini Pressure Reactor for HPTH Pretreatment ........................................... 20

Figure 3.5 Daily Operation of the Biological Reactor..................................................................... 22

Figure 3.6 Daily Operation of the Aerobic Digester (day 77 to 283) .............................................. 24

Figure 3.7 Daily Pretreatment Procedure (day 203 to 283 )............................................................ 25

Figure 3.8 Overall Framework for Characterization of Impacts of HPTH Pretreatment on WAS ...... 26

Figure 3.9 Project Timeline .................................................................................................... 27

Figure 3.10 DO Probe and Analyzer Connected to Aerators in the AD ............................................32

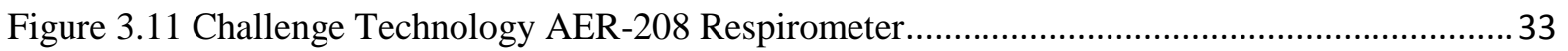

Figure 4.1 Simulated COD Fractionation of WAS in BR during Startup ......................................... 36

Figure 4.2 COD Fractionation of WAS in BR Over 20 Days ........................................................ 37

Figure 4.3 COD Fractionation of Synthetic Wastewater.............................................................. 39

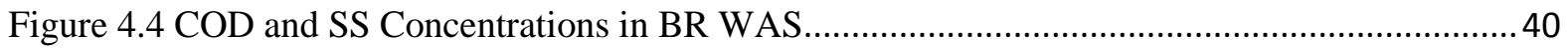

Figure 4.5 Measured pH in Raw and Pretreated BR WAS........................................................... 43

Figure 4.6 Average COD Concentrations in Raw and Pretreated BR WAS ..................................... 44

Figure 4.7 Average SS Concentrations in Raw and Pretreated BR WAS .........................................45

Figure 4.8 Average Concentration of Nitrogen Species in Raw and Pretreated BR WAS .................46

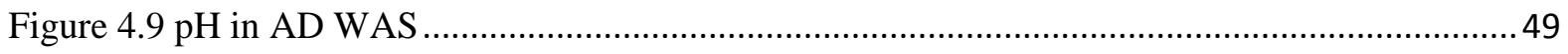

Figure 4.10 Average COD Concentrations in Phase 1 and 2 AD WAS ............................................... 50

Figure 4.11 COD and SS Concentrations in AD WAS ............................................................... 51

Figure 4.12 Average SS Concentrations in Sludge Streams in Phases 1 and 2 ................................52

Figure 4.13 Typical DO Concentration in AD and OUR Calculation from Rate of DO Decline ........ 54

Figure 4.14 Typical OUR in AD ........................................................................................... 55

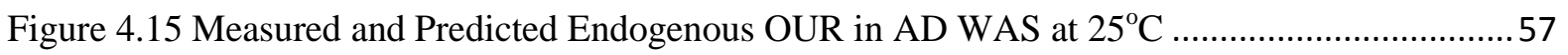

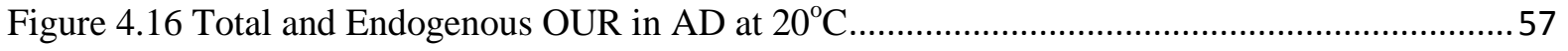

Figure 4.17 Typical Phase 1 OUR in BR WAS, AD WAS and Inoculated BR WAS ........................59

Figure 4.18 Typical Phase 2 OUR in Pretreated BR WAS, AD WAS and Inoculated Pretreated BR

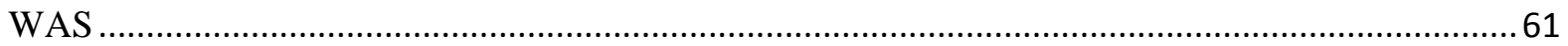

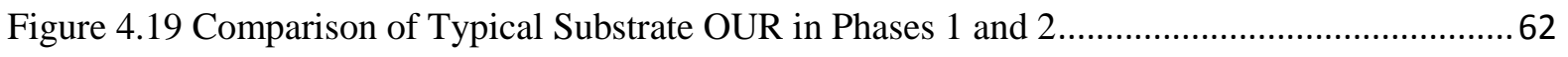

Figure $4.20\left(\mathrm{TCOD}_{\mathrm{i}}-\mathrm{TCOD}_{\mathrm{f}}\right) / \mathrm{TCOD}_{\mathrm{i}}$ Ratio from Offline Respirometry on Substrate.....................63

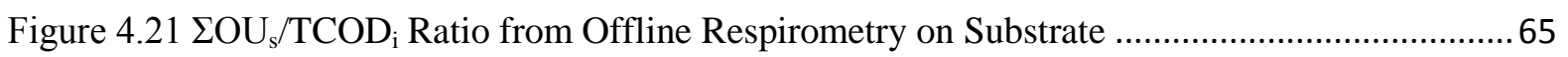

Figure 4.22 Typical Respirometry Data Used to Estimate $Z_{\mathrm{bh}, 0}$ (a) Total Respirogram (b)

Exponentially Increasing Portion of Respirogram (c) Log of Exponentially Increasing Portion ........ 69

Figure 4.23 COD Fractionation of Sludge Streams.................................................................... 72

Figure 5.1 Configuration of Phase 1 BR-AD System in BioWin ${ }^{\circledR} 3.1$ Platform................................ 77 
Figure 5.2 Measured and Simulated OUR from Offline Respirometry on BR WAS

Figure 5.3 Measured and Simulated OUR from Offline Respirometry on Inoculated BR WAS and AD WAS

Figure 5.4 Measured and Simulated OUR from Online Respirometry in Phase 1 AD ......................8 88

Figure 5.5 Configuration of Switchover from Phase 1 to 2 in BioWin ${ }^{\circledR}$ 3.1 Platform ........................ 89

Figure 5.6 Measured and Simulated OUR from Offline Respirometry on Inoculated Pretreated BR WAS .94

Figure 5.7 Measured and Simulated OUR from Online Respirometry in Phase 2 AD ...................... 95

Figure 5.8 Configuration of Phase 2 System in BioWin® ${ }^{\circledR}$ 3.1 Platform ........................................... 97

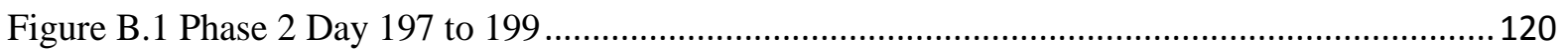

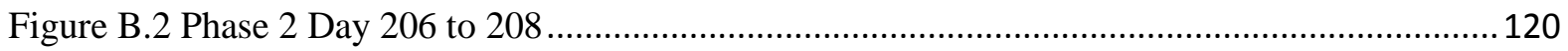

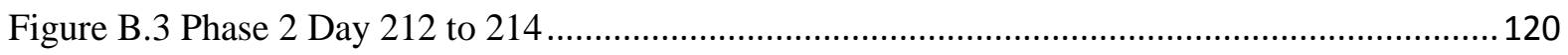

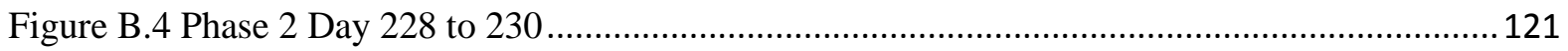

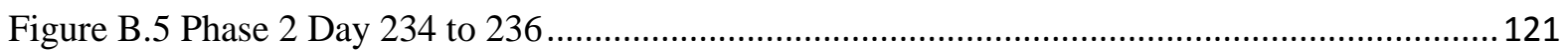

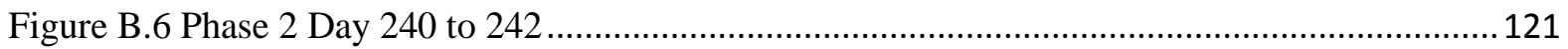

Figure B.7 Phase 2 Day 246 to 248 ........................................................................................ 122

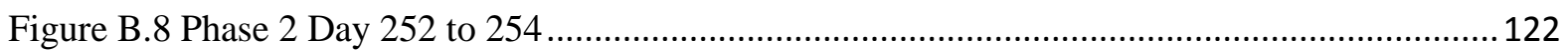

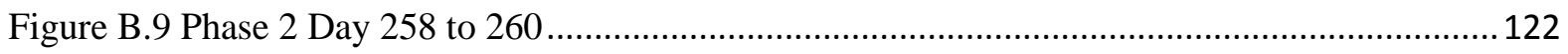

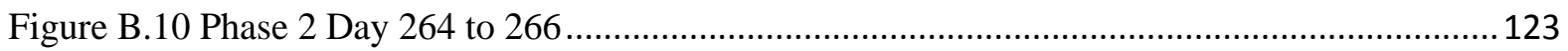

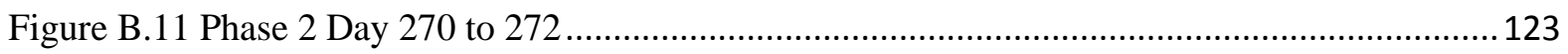

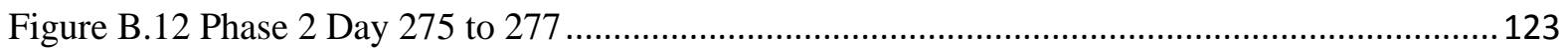

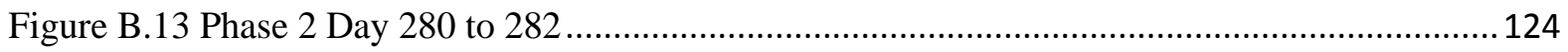

Figure B.14 Measured $\Sigma \mathrm{OU}_{\mathrm{s}} / \mathrm{TCOD}_{\mathrm{i}}$ Ratios of BR WAS Fed to AD ............................................ 124

Figure C.1 BR WAS and AD WAS - Test Started on Day 193 ................................................... 126

Figure C.2 BR WAS and AD WAS - Test Started on Day 203 ….............................................. 126

Figure C.3 Pretreated BR WAS and Non-acclimatized AD WAS - Test Started on Day 197 .......... 127

Figure C.4 Pretreated BR WAS and Acclimatized AD WAS - Test Started on Day 215 ................127

Figure C.5 Pretreated BR WAS and Acclimatized AD WAS - Test Started on Day 222 ................ 128

Figure C.6 Pretreated BR WAS and Acclimatized AD WAS - Test Started on Day 228 ................ 128

Figure C.7 Pretreated BR WAS and Acclimatized AD WAS - Test Started on Day 236 ................ 129

Figure C.8 Pretreated BR WAS and Acclimatized AD WAS - Test Started on Day 243 ................ 129

Figure C.9 Pretreated BR WAS and Acclimatized AD WAS - Test Started on Day 250 ................ 130

Figure C.10 Pretreated BR WAS and Acclimatized AD WAS - Test Started on Day 271 .............. 130 


\section{List of Tables}

Table 3.1 Organic Synthetic Wastewater ................................................................................. 21

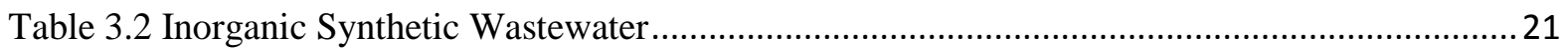

Table 3.3 Sampling Schedule for Physical and Biochemical Tests.................................................. 27

Table 3.4 Sample Bottle Contents for Offline Respirometric Tests .................................................. 33

Table 4.1 Influent Synthetic Wastewater Parameters................................................................... 36

Table 4.2 Concentration of Conventional Parameters in Synthetic Wastewater ............................... 38

Table 5.1 ASM3 Model in BioWin ${ }^{\circledR}$ Platform Showing Processes in Phase 1 BR-AD System ..........80

Table 5.2 Kinetic and Stoichiometric Parameters in ASM3 Model for Phase 1 BR-AD System ........ 81

Table 5.3 Measured and Simulated COD, SS and N Species in BR WAS ....................................... 83

Table 5.4 Measured and Simulated COD, SS and N Species in Phase 1 AD WAS............................. 83

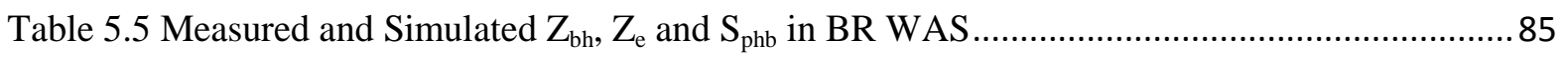

Table 5.6 Measured and Simulated $Z_{\mathrm{bh}}, Z_{\mathrm{e}}$ and $\mathrm{S}_{\mathrm{phb}}$ in Phase 1 AD WAS ......................................... 85

Table 5.7 Measured and Simulated COD Species in Pretreated BR WAS ....................................... 91

Table 5.8 Measured and Simulated COD, SS and N Species in Phase 2 AD WAS............................ 92

Table 5.9 Measured and Simulated $\mathrm{Z}_{\mathrm{bh}}, \mathrm{Z}_{\mathrm{e}}$ and $\mathrm{S}_{\mathrm{phb}}$ in Phase 2 AD WAS ..................................... 93

Table 5.10 Simulated COD Fractionation of BR WAS and Pretreated BR WAS ............................97

Table 5.11 Pretreatment Model .................................................................................................. 97

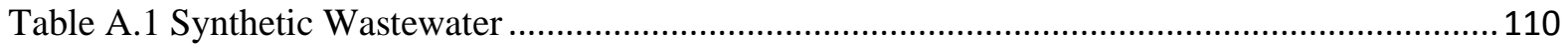

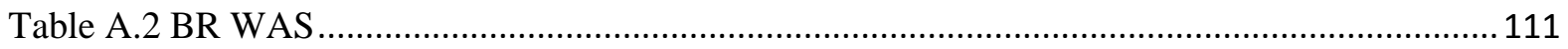

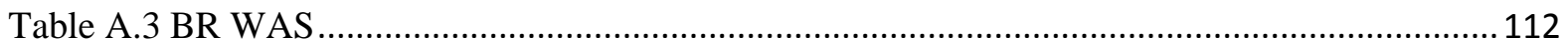

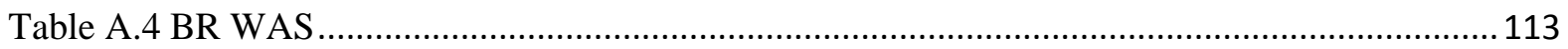

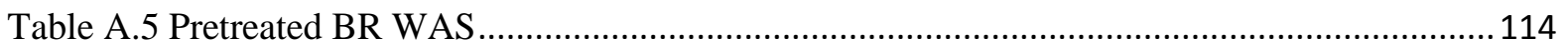

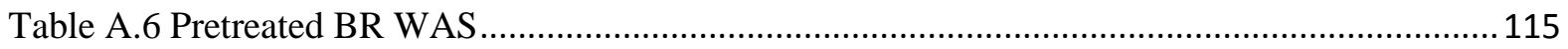

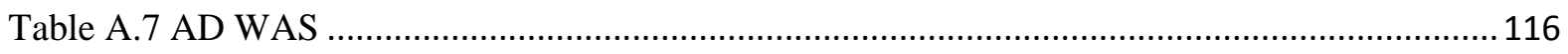

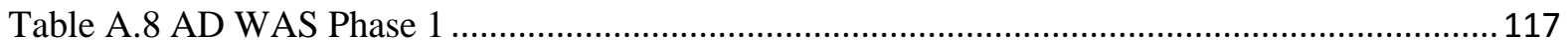

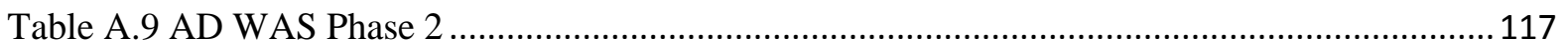

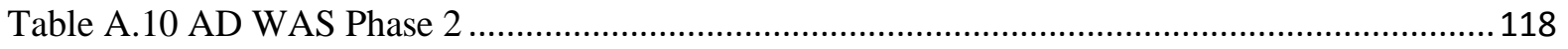




\section{List of Abbreviations}

\begin{tabular}{|c|c|}
\hline AD & Aerobic Digester \\
\hline ADM1 & Anaerobic Digestion Model Number 1 \\
\hline ASM3 & IWA Activated Sludge Model Number 3 \\
\hline AS & Activated Sludge \\
\hline ASM & IWA Activated Sludge Model \\
\hline ASM1 & IWA Activated Sludge Model Number 1 \\
\hline bCOD & Biodegradable COD \\
\hline $\mathbf{b}_{\mathrm{h}}$ & Aerobic Decay Rate of $Z_{b h}$ \\
\hline $\mathbf{B R}$ & Biological Reactor \\
\hline COD & Chemical Oxygen Demand \\
\hline DO & Dissolved Oxygen \\
\hline EPS & Extracellular Polymeric Substances \\
\hline ffCOD & Filtered and Flocculated COD \\
\hline HPTH & High Pressure Thermal Hydrolysis \\
\hline HRT & Hydraulic Retention Time \\
\hline ISS & Inorganic Settleable Solids \\
\hline IWA & International Water Association \\
\hline LCFA & Long Chain Fatty Acid \\
\hline MLSS & Mixed Liquor Suspended Solids \\
\hline MLVSS & Mixed Liquor Volatile Suspended Solids \\
\hline MOE & Ontario Ministry of the Environment \\
\hline nbCOD & Non-Biodegradable COD \\
\hline $\mathbf{N H}_{3}-\mathbf{N}$ & Ammonia Nitrogen \\
\hline $\mathrm{NO}_{3}-\mathrm{N}$ & Nitrate Nitrogen \\
\hline NOUR & Nitrogenous Oxygen Uptake Rate \\
\hline$\Sigma O U$ & Cumulative Oxygen Uptake \\
\hline OUR & Oxygen Uptake Rate \\
\hline PAA & Peracetic acid \\
\hline PAO & Phosphate Accumulating Organism \\
\hline PCOD & Particulate Chemical Oxygen Demand \\
\hline PHA & Poly-hydroxy-alkanoates \\
\hline PS & Primary Sludge \\
\hline PT & Pretreatment \\
\hline RAS & Return Activated Sludge \\
\hline rbCOD & Readily Biodegradable COD \\
\hline
\end{tabular}




\begin{tabular}{|c|c|}
\hline sbCOD & Slowly Biodegradable COD \\
\hline SBR & Sequencing Batch Reactor \\
\hline $\mathbf{S}_{\mathrm{bsa}}$ & Readily Biodegradable COD (acetate) \\
\hline $\mathbf{S}_{\text {bsc }}$ & Readily Biodegradable COD (complex) \\
\hline SCa & Dissolved Calcium Concentration \\
\hline sCOD & Soluble COD \\
\hline SMg & Dissolved Magnesium Concentration \\
\hline $\mathbf{S}_{\mathbf{p h b}}$ & Stored COD \\
\hline SRT & Solids Residence Time \\
\hline sON & Soluble Organic Nitrogen \\
\hline SRT & Solids Residence Time \\
\hline SST & Secondary Settling Tank \\
\hline sTKN & Soluble Total Kjeldahl Nitrogen \\
\hline $\mathbf{S}_{\mathrm{us}}$ & Soluble Inert COD \\
\hline TCOD & Total Chemical Oxygen Demand \\
\hline TKN & Total Kjeldahl Nitrogen \\
\hline $\mathbf{T P}$ & Total Phosphorous \\
\hline TSS & Total Settleable Solids \\
\hline VFA & Volatile Fatty Acids \\
\hline VS & Volatile Solids \\
\hline VSS & Volatile Settleable Solids \\
\hline WAS & Waste Activated Sludge \\
\hline WW & Wastewater \\
\hline $\mathbf{X}_{\mathbf{i}}$ & Particulate Inert COD \\
\hline $\mathbf{X}_{\mathrm{sc}}$ & Slowly Biodegradable COD (colloidal) \\
\hline $\mathbf{X}_{\mathrm{sp}}$ & Slowly Biodegradable COD (particulate) \\
\hline $\mathbf{X}_{\text {STO }}$ & Stored COD \\
\hline $\mathbf{Y}_{\mathbf{h}}$ & Aerobic Yield of $\mathrm{Z}_{\mathrm{bh}}$ \\
\hline $\mathbf{Z}_{\mathrm{bh}}$ & Heterotrophic Microorganisms \\
\hline $\mathbf{Z}_{\mathrm{e}}$ & Endogenous Products \\
\hline$\mu_{\max }$ & Specific Maximum Growth Rate \\
\hline
\end{tabular}




\section{Introduction}

The main by-product of biological wastewater treatment is waste activated sludge (WAS). The use of biological wastewater treatment and hence the generation of WAS is increasing with population growth and the need to sustain water resources (Reynolds and Richards, 1996). At the same time, sludge disposal to agricultural lands is becoming increasingly limited as sanitation standards become more stringent (Henze et al., 2008). The sludge must be adequately stabilized and sterilized and its production minimized.

WAS is typically stabilized by biological digestion to reduce pathogens, eliminate offensive odours and reduce the potential for putrefaction (Tchobanoglous et al., 2003). In a time of continually rising energy costs, anaerobic digestion is preferred over aerobic digestion because it requires substantially less energy and generates methane which can be used as fuel. Furthermore, anaerobic digestion produces a lower biomass yield than aerobic digestion, thereby reducing the energy demand associated with further processing or transporting the sludge. However the significant disadvantage of anaerobic digestion is that it requires a long digestion time. The anaerobic digestion process is composed of four stages: hydrolysis, acidogenesis, acetogenesis and methanogenesis (Henze et al., 2008). The hydrolytic reactions are considered to be the rate limiting step in the overall process ( $\mathrm{Li}$ and Noike, 1992; Shimizu et al., 1993).

Recent research has shown that various WAS pretreatment technologies have the potential to enhance the digestibility of the sludge, thereby reducing the required anaerobic digestion time. In general, pretreatment causes the bacterial membranes in the WAS to rupture. As a result, organic substances and nutrients are released. These organics are more easily hydrolyzed (Wang et al., 1999). A wide range of promising WAS pretreatment methods are currently being developed and a growing number of full-scale installations exist. These technologies can be broadly categorized as thermal (Camacho et al., 2002; Donoso-Bravo et al., 2010 b; Tattersall et al., 2011), chemical (Deleris and Rouston, 2000; Saby et al., 2002; Tanaka and Kamiyama, 2002; Appels et al., 2010), biological (Neyens and Baeyens, 2003) or mechanical (Tiehm et al., 2001; Chu et al., 2002; Braguglia et al., 2008) disintegration, with some technologies falling under more than one category (Musser, 2009).

In order to characterize the impact of pretreatment on WAS, a variety of indicators have been employed. Many of these indicators are based on the associated improvement in plant performance. For example, pretreatment has been shown to enhance methane generation during anaerobic digestion 
(Wang et al., 1995). These plant-performance indicators are however, heavily dependent on the raw sludge composition and configuration of WWTPs. Hence it is difficult to apply the results of existing pretreatment studies to waste streams different from the stream used in the original study. Furthermore, it has been shown that some pretreatment doses such as ozonation and sonication are challenging to quantify (Musser, 2009). A generally accepted means of characterizing and comparing all pretreatment processes does not exist.

An essential design tool of biological wastewater treatment is the activated sludge model. The International Water Association (IWA) has sponsored the development of standardized activated sludge model platforms. The first activated sludge model, the IWA Activated Sludge Model Number 1 (ASM1), was developed in 1987. Since then increasingly more complex activated sludge models have been designed (Henze et al., 2008). The concentrations of all organic components in the IWA activated sludge models are given in units of chemical oxygen demand (COD). The COD is a consistent measure of the energy that is present in the wastewater. In the ASMs, the organic wastewater constituents are separated into biodegradable versus non-biodegradable and soluble versus particulate COD fractions. The development of a COD-based model that could describe WAS pretreatment would provide a means of comparing all types and doses of WAS pretreatment.

\subsection{Motivation}

WAS pretreatment is typically evaluated in terms of the associated improvement in sludge digestion and biogas production. However, WAS properties and hence the impact of pretreatment on WAS properties depend on the raw sludge composition and configuration of WWTPs. A generally accepted means of characterizing and comparing all pretreatment processes does not exist. The motivation for this project was to show the potential of using COD fractionation as a universal characterizer of WAS pretreatment.

The most extensively researched and widely used WAS pretreatment technique is thermal pretreatment, in particular high pressure thermal hydrolysis (HPTH). Full-scale installations of this type have been successfully used for more than a decade (Tattersall et al., 2011). This type of pretreatment has the potential to produce Class A biosolids as defined by United States CFR 40 Part 503.32 (USEPA, 1999). Class A biosolids contain no detectable levels of pathogens. In general, Class A biosolids may be used in small quantities by the public without buffer requirements or 
restrictions on crop type, crop harvesting or site access. HPTH pretreatment is of great interest to the wastewater industry because it holds the combined benefits of decreasing the biosolids production while improving the acceptability of biosolids (Wilson and Novak, 2009). Based on its popularity and proven potential, HPTH pretreatment was selected to be used in this project.

\subsection{Objectives}

This project aimed to:

- Fractionate the COD of raw and pretreated WAS using analytical and bioassay methods to show how pretreatment may increase the rate and extent of aerobic digestion.

- Develop a COD-based pretreatment model.

- Identify any limitations of the pretreatment model and recommend ways to improve the model

\subsection{Scope}

This project investigated the impacts of thermal waste activated sludge (WAS) pretreatment in a bench-scale system and the experimental results were used to develop a pretreatment model. The scope of this project included:

- Operation of a bench-scale biological reactor (BR) initially seeded with activated sludge from the Waterloo WWTP, and then fed daily with synthetic wastewater to generate a stable source of WAS

- Operation of a bench-scale aerobic digester (AD) fed daily with BR WAS in Phase 1, and then fed daily with thermally pretreated BR WAS in Phase 2

- Characterization of the raw and pretreated BR WAS and the Phase 1 and 2 AD WAS with respect to $\mathrm{pH}$, suspended solids and $\mathrm{COD}$ and nitrogen species

- Assessment of the biodegradability and concentrations of active biomass and endogenous decay products of the various sludge streams by online and offline respirometric methods

- Simulation of the Phase 1 and 2 systems using an activated sludge model calibrated with measured data 
- Development of a COD-based thermal pretreatment model and integration of the model into a wastewater treatment plant (WWTP) simulator 


\section{Background}

\subsection{Thermal Pretreatment}

\subsubsection{Introduction}

Thermal pretreatment has been studied using a wide range of temperatures ranging from $60^{\circ} \mathrm{C}$ to $270^{\circ} \mathrm{C}$ (Climent et al., 2007). Pretreatment at temperatures under $100^{\circ} \mathrm{C}$ is considered low temperature (LT) pretreatment. Pretreatment at temperatures above $100^{\circ} \mathrm{C}$ is accompanied by elevated pressure and is referred to as high pressure thermal hydrolysis (HPTH).

Research suggests that LT pretreatment enhances the bioactivity of some thermophilic bacterial populations with an optimum activity at temperatures around $70^{\circ} \mathrm{C}$ (Nielsen et al., 2004). Although LT pretreatment requires considerably less energy input than HPTH, previous studies have shown that LT pretreatment is ineffective in improving sludge digestion. Nielsen et al. (2011) reported that pretreatment at $80^{\circ} \mathrm{C}$ had no effect on methane yield in an anaerobic digester. In a study carried out by Prorot et al. (2011), samples were tested at a constant heating time of 20 minutes while the heating temperature was varied from $20^{\circ} \mathrm{C}$ to $95^{\circ} \mathrm{C}$. These researchers found that the cumulative biogas production in anaerobic conditions was very similar for both untreated and heat treated samples. Therefore, low temperature heat treatment did not seem to improve the anaerobic biodegradability of the sludge.

HPTH pretreatment is much more promising than LT pretreatment. HPTH disintegrates cells which releases intracellular matter that is more accessible to anaerobic microorganisms (Gurieff et al., 2011). This in turn reduces the sludge viscosity which eases the mixing of the digester. Without good mixing, the solids settle in the digester and hence reduce the active volume. Reducing the sludge viscosity therefore allows digesters to be operated at substantially higher organic loading rates (Morgan-Sagasume et al., 2010). Furthermore HPTH produces volatile fatty acids (VFAs) and it has been found that the ratio of acetic to propionic acid in fermented HPTH is very similar to that needed for biological phosphorous removal. Thus the dewatered digested sludge could potentially be recycled to the anaerobic reactor to promote the growth of polyphosphate accumulating organisms (Morgan-Sagasume et al., 2010). 
HPTH is becoming established worldwide as an effective sludge pretreatment technique due to its capacity to deliver Class A biosolids and enhance VSS reduction and methane production (Tattersall et al., 2011). Producing Class A biosolids, as defined by the USEPA, provides more diverse disposal options (USEPA, 1999). Several studies have shown that the increase in biogas production could be sufficient to preheat the WAS and heat the anaerobic digesters (Bougrier et al., 2007; Phothilangka et al., 2008; Gurieff et al., 2011). The high temperatures are typically applied by heat exchangers or steam injection (Climent et al., 2007). All these listed benefits provide significant operational cost savings. The remainder of this chapter will focus on HPTH pretreatment, the selected type of pretreatment for this project.

\subsubsection{Doses}

Most HPTH pretreatment methods involve heating the sludge to temperatures in the range of 140 to $200^{\circ} \mathrm{C}$ under a corresponding pressure of 3 to 25 bars. Numerous studies have shown that within this temperature range, the duration of heating has less effect on the sludge properties than the treatment temperature (Bougrier et al., 2007; Climent et al., 2007). The common heating time for HPTH pretreatment is 30 to 60 minutes. Dwyer et al. (2008) showed that the organic matter became more solubilized when the reaction temperature was increased from $140^{\circ} \mathrm{C}$ to $165^{\circ} \mathrm{C}$. However, they monitored the soluble residuals after digestion, overall methane formation and hydrolysate organic acid concentration and showed that the additional solubilized materials were not degradable. Furthermore, the degradation rate did not change substantially over the range $140^{\circ} \mathrm{C}$ to $165^{\circ} \mathrm{C}$ (Dwyer et al., 2008). Several reports have shown that HPTH pretreatment polymerizes low molecular weight intermediate compounds such as carbohydrates and amino acids to produce coloured recalcitrant refractory compounds (Bougrier et al., 2007; Climent et al., 2007; Dwyer et al., 2008; Donoso-Bravo et al., $2010 \mathrm{~b}$ ). Studies have reported that refractory compounds begin to form at temperatures as low as $150^{\circ} \mathrm{C}$ to as high as $190^{\circ} \mathrm{C}$. These refractory compounds are undesirable because they will contribute soluble non-biodegradable COD that could add to the treatment plant effluent stream if dewatering streams are recycled.

HPTH pretreatment techniques have been commercialized and are in full-scale operation (MorganSagasume et al., 2010). The most well-known is $\mathrm{CAMBI}^{\mathrm{TM}}$ which has more than 25 installations worldwide (Abu-Orf and Goss, 2011). $\mathrm{CAMBI}^{\mathrm{TM}}$ reactors are operated in batch mode whereby the sludge temperature is initially heated to $80^{\circ} \mathrm{C}$, followed by thermal hydrolysis at $165^{\circ} \mathrm{C}$ at 7 bars for 
30 minutes. The sludge then enters a flash tank where the steam is released and used to preheat the raw sludge (Abu-Orf and Goss, 2011). Besides CAMBI ${ }^{\mathrm{TM}}$, the only other commercial HPTH pretreatment technique that has been proven to produce Class A biosolids is Exelys ${ }^{\mathrm{TM}}$. Up to now, only one full-scale Exelys ${ }^{\mathrm{TM}}$ pilot plant is in operation (at Hillerod, Denmark). The Exelys ${ }^{\mathrm{TM}}$ process operates at the same temperature, pressure and retention time as $\mathrm{CAMBI}^{\mathrm{TM}}$. However unlike $\mathrm{CAMBI}^{\mathrm{TM}}$, Exelys ${ }^{\mathrm{TM}}$ is a continuous plug flow system that uses a series of batch tanks and does not include a flash period (Gurieff et al., 2011). It has been suggested that the flash period assists in disintegrating the sludge however Gurieff et al. (2011) showed that eliminating the flash period did not affect the COD solubilization.

Based on the results of existing HPTH pretreatment research, a conservative temperature of $150^{\circ} \mathrm{C}$ was selected for this study with a corresponding pressure of 3 bars. Heating the sludge at this temperature was expected to improve the degradability while minimizing the generation of soluble non-biodegradable COD. The selected heating duration was 30 minutes which is similar to the $\mathrm{CAMBI}^{\mathrm{TM}}$ and Exelys ${ }^{\mathrm{TM}}$ processes.

\subsubsection{Physical Properties}

In the current project, the impact of HPTH pretreatment on the physical properties of WAS was assessed by monitoring the TSS and VSS. This section presents a brief literature review of the impact of HPTH pretreatment on these parameters.

Bougrier et al. (2008) pretreated five different WAS samples for 30 minutes at temperatures ranging from 90 to $210^{\circ} \mathrm{C}$ in a laboratory autoclave. The authors showed that the VSS/TSS ratio decreased with the treatment temperature indicating that the particulates became more mineral in nature. At $150^{\circ} \mathrm{C}$, the average VSS/TSS ratio was $72 \%$ whereas the ratio was $81 \%$ for untreated sludge. In another study, the performance of the $\mathrm{CAMBI}^{\mathrm{TM}}$ process was evaluated at three full-scale WWTPs (Morgan-Sagasume et al., 2010). The authors showed that the $\mathrm{CAMBI}^{\mathrm{TM}}$ process caused a decrease in TSS of 20 to $30 \%$ demonstrating that HPTH pretreatment solubilizes suspended matter. In a third study, Gurieff et al. (2011) operated an Exexlys ${ }^{\mathrm{TM}}$ pilot plant over a 9 month period and showed that the average VSS solubilization was $31 \%$. In this study, the VSS solubilization was calculated by equation 2.1: 


$$
\text { VSS solubilization } \%=\frac{V S S_{i}-V S S_{f}}{T S} \times 100 \%
$$

In equation 2.1, VSS $\mathrm{V}_{\mathrm{i}}$ and $\mathrm{VSS}_{\mathrm{f}}$ were the respective VSS concentrations before and after pretreatment and TS was the total solids concentration.

\subsubsection{Biochemical Properties}

In the current project, $\mathrm{pH}, \mathrm{COD}$ and nitrogen species were measured in order to assist in fractionating the COD of the WAS before and after pretreatment. Recently published related studies have considered the impact of HPTH pretreatment on these particular biochemical indicators. The extent of solubilization of COD and nitrogen species may be used to assess the impact of pretreatment on the biodegradability of WAS (Kianmehr, 2010). Solubilized materials are potentially more easily hydrolyzed than particulate materials. The results of four HPTH studies are compared here.

The desired $\mathrm{pH}$ range for anaerobic digestion is 6.6 to 8.2 (Parker, 2010). Therefore it is important to assess the impact of HPTH pretreatment on the $\mathrm{pH}$ of WAS to determine whether $\mathrm{pH}$ adjustment is required before feeding the WAS to the anaerobic digester. Bougrier et al. (2008) showed that the pH of the pretreated WAS increased from 6.9 at $90^{\circ} \mathrm{C}$ to 7.3 at $150^{\circ} \mathrm{C}$ then decreased to 6.8 at $170^{\circ} \mathrm{C}$. The authors postulated that the $\mathrm{pH}$ increase was due to protein desorption or acidic compound volatilization. They suggested that the $\mathrm{pH}$ decrease could be linked to the degradation of macromolecules into acidic compounds. Morgan-Sagasume et al. (2010) showed that the CAMBI ${ }^{\mathrm{TM}}$ process slightly decreased the $\mathrm{pH}$ of the WAS from an average of 6.7 to 6.2. The results of these two studies indicate that HPTH at temperatures around 165 to $170^{\circ} \mathrm{C}$ slightly decreases the $\mathrm{pH}$ of the WAS.

By far the most common indicator employed by the HPTH pretreatment studies referenced in this project is the COD solubilization caused by pretreatment. In the four studies presented below, COD solubilization was calculated with equation 2.2:

$$
\text { COD solubilization } \%=\frac{S C O D_{f}-S C O D_{i}}{P C O D_{i}} \times 100 \%
$$

In this formula, $\mathrm{SCOD}_{\mathrm{i}}$ and $\mathrm{SCOD}_{\mathrm{f}}$ were the respective $\mathrm{SCOD}$ concentrations measured before and after pretreatment and $\mathrm{PCOD}_{\mathrm{i}}$ was the initial particulate COD. Bougrier et al. (2008) showed that COD solubilization increased linearly with temperature from $90^{\circ} \mathrm{C}$ up to $200^{\circ} \mathrm{C}$. The reported average COD solubilization at $150^{\circ} \mathrm{C}$ was $40 \%$. Donoso-Bravo et al. (2010 b) carried out lab-scale and pilot- 
scale HPTH pretreatment experiments on WAS in which the temperature and pressure were held constant at $170^{\circ} \mathrm{C}$ and 8 bars respectively while the contact time was varied. This study showed that the SCOD concentration increased with contact time up to 15 minutes but there were no additional changes for a contact time of 30 minutes. The COD solubilization was $45 \%$ at 30 minutes. In a $\mathrm{CAMBI}^{\mathrm{TM}}$ pretreatment study, Morgan-Sagasume et al. (2010) reported that the average COD solubilization was 39\%. These authors also reported that both the TCOD and TN concentrations remained unchanged by pretreatment, indicating that no significant degradation or removal of organic matter occurred. Therefore, the HPTH process did not diminish the available resource for methane production. In an Exexlys ${ }^{\mathrm{TM}}$ pretreatment study, Gurieff et al. (2011) reported that the average COD solubilization was $28 \%$. Hence it can be observed that the COD solubilization results of these four studies were comparable and ranged from 28 to $45 \%$.

In terms of changes in the fractionation of nitrogen compounds, Bougrier et al. (2008) reported that the ammonia concentration increased with temperature up to $90^{\circ} \mathrm{C}$, then remained constant at higher temperatures. During thermal pretreatment, proteins may be broken down to polypeptides which may in turn be broken down to amino acids. The amino acids may then be mineralized, i.e. cleaved to release ammonia. The results of Bougrier et al. (2008) indicate LT pretreatment mineralized proteins. It is highly likely that further protein degradation occurred at the higher temperatures tested however mineralization did not occur above $90^{\circ} \mathrm{C}$. Donoso-Bravo et al. (2010 b) reported that HPTH only slightly increased the ammonia concentration, demonstrating marginal protein mineralization. Morgan-Sagasume et al. (2010) found that the mass of total nitrogen per mass of total solids in the sludge remained constant during thermal pretreatment. Because the mass of TCOD per mass of total solids and mass of total phosphorous per mass of total solids also remained constant during pretreatment, these authors concluded that no significant degradation of organic matter occurred. As previously discussed, these same studies showed that HPTH solubilized COD. It can therefore be concluded that proteins were solubilized rather than mineralized by HPTH.

\subsubsection{Biological Properties}

The biological properties of WAS such as the activity of the biomass and biodegradability of the sludge are expected to be impacted by pretreatment. In this part of the literature review, the biological impacts of HPTH pretreatment are discussed and the indicators that describe such impacts are introduced. 


\subsubsection{Activity of Bacteria}

The inactivation of bacteria in WAS caused by HPTH may be indicative of the conversion of biomass into a more readily biodegradable form (Kianmehr, 2010). Disrupted, dead and inactive biomass may be disintegrated and hydrolyzed easier than active biomass. The inactivation of biomass may also change the nature of the flocs and facilitate their disintegration. Donoso-Bravo et al. (2010 b) measured the total coliforms in the sludge before and after pretreatment at $170^{\circ} \mathrm{C}$ and 8 bars over a range of contact periods. They found undetectable levels in every pretreated sludge sample. Gurieff et al. (2011) tested samples before and after pretreatment by the Exelys ${ }^{\mathrm{TM}}$ process for fecal coliforms, Enterococci and helminth eggs. Unlike the $\mathrm{CAMBI}^{\mathrm{TM}}$ process that is operated in batch mode, the Exelys ${ }^{\mathrm{TM}}$ process is continuous and it is possible that some organisms may short-circuit the process and remain viable. However they showed that the remaining organisms were below the detection limit. It must be noted that the measurement of viable microorganisms in these studies was employed to determine whether the sludge could be considered sterile as defined by the USEPA for Class A biosolids. The culturable microorganisms measured in these studies probably only comprised a small fraction of the total active heterotrophs present.

A much more accurate method to measure the concentration of active heterotrophic bacteria is to use batch-mode respirometry. In this method, the oxygen uptake rate (OUR) is determined over a period of time by measuring the consumption of dissolved oxygen in the WAS sample in an airtight vessel. In the current project, the active fraction is defined as the ratio of initial active biomass (mg COD/L) measured by respirometry to TCOD (mg COD/L). Two approaches will be presented to determine the active fraction from OUR time-series data.

In the first approach, a small volume of WAS is combined with a comparably large volume of substrate in the batch respirometric test such that the food to microorganism (F/M) ratio is greater than 10. The measured OUR will increase exponentially with time, indicating that the bacteria are reproducing. Once the substrate is depleted, the measured OUR will decrease with time. Both Kianmehr (2010) and Musser (2009) successfully employed this approach to measure the concentration of active bacteria in WAS before and after pretreatment by sonication and ozonation. Wentzel et al. (1998) used equation 2.3 to determine the initial active biomass concentration $\left(Z_{\mathrm{bh} 0}\right)$ in $\mathrm{mg} \mathrm{COD/L}$ on the basis of respirometry data:

$$
Z_{b h 0}=\frac{e^{y-\text { intercept }}}{\frac{1-Y_{h}}{Y_{h}} \times \text { slope } \times b_{h}}
$$


In this formula, the slope and y-intercept are of the plot of $\ln (\mathrm{OUR})$ versus time (d) for the portion of the measured OUR data that exponentially increases with time. The measured OUR is in units of mg $\mathrm{COD} / \mathrm{L} / \mathrm{d}$. The aerobic decay rate is represented as $\mathrm{b}_{\mathrm{h}}\left(\mathrm{d}^{-1}\right)$ and the aerobic yield coefficient is represented as $\mathrm{Y}_{\mathrm{h}}$. Kianmehr (2010) reported that estimates of $\mathrm{Z}_{\mathrm{bh} 0}$ were not significantly sensitive to the value of $b_{h}$ and so a value of $0.24 \mathrm{~d}^{-1}$ at $20^{\circ} \mathrm{C}$ was used in the current project unless otherwise stated. This is the value of $b_{h}$ recommended by Henze et al. (2008). The typical value of $Y_{h}$ in real activated sludge systems is 0.67 (Henze et al., 2008). In activated sludge systems fed by sodium acetate as the sole carbon source $Y_{h}$ values of 0.6 have been reported (Ramdani et al., 2012). Unless otherwise stated, the $\mathrm{Y}_{\mathrm{h}}$ value used in the current project was 0.6.

The second approach uses only WAS in a batch respirometric test. The measured OUR will decrease exponentially with time, indicating that endogenous decay is the only oxygen-consuming process in the vessel. A nonlinear regression fit of equation 2.4 to the measured OUR data will yield an estimate of $\mathrm{Z}_{\mathrm{bh} 0}$ in a sample (Jones et al., 2009).

$$
\text { OUR }=\left(4.33 f_{N}+1\right)(1-f) b_{h} Z_{b h 0} e^{-b_{h} t}\left(\frac{1}{24}\right)
$$

Where $\quad$ OUR $=$ measured oxygen uptake rate $\left(\mathrm{mg} \mathrm{O}_{2} / \mathrm{L} / \mathrm{h}\right)$

$\mathrm{f}_{\mathrm{N}}=$ nitrogen content of heterotrophic organisms

$\mathrm{f}=$ endogenous decay product fraction of organisms (0.2)

$\mathrm{t}=$ time (days)

In systems where nitrification is suppressed, the $4.33 \mathrm{f}_{\mathrm{N}}$ term is omitted to eliminate the nitrogenous OUR (NOUR); the measured OUR is entirely carbonaceous OUR (COUR).

\subsubsection{Biodegradability}

HPTH pretreatment may impact the rate and extent of biodegradability of the WAS. As demonstrated by Kianmehr (2010), the anaerobic biodegradability of the WAS may be measured using the biochemical methane potential (BMP) test or the biochemical acidogenic potential (BAP) test however these tests were outside the scope of this study. This project focused on measuring the impact of HPTH on the aerobic degradability of the WAS using respirometric methods. Several WAS pretreatment studies have employed respirometry to estimate the readily and slowly biodegradable COD concentrations in raw and pretreated WAS (Spanjers and Vanrolleghem, 1995; Mathieu and Etienne, 2000; Musser, 2009; Kianmehr, 2010). Readily biodegradable COD is typically soluble whereas slowly biodegradable COD is particulate. Since HPTH pretreatment has 
been shown to solubilize organic materials, an increase in the level of readily biodegradable COD (rbCOD) or rate of aerobic biodegradability is expected when HPTH pretreatment is applied.

Musser (2009) analyzed the effects of sonication and ozonation on the aerobic biodegradability of authentic WAS using respirometry. F/M ratios between 0.1 and 10 were employed where neither the growth nor the decay processes were dominant. Musser (2009) estimated the readily and slowly biodegradable COD concentrations by fitting OUR responses predicted by ASM1 to measured respirometric data. OUR curves generated using ASM1 showed three distinct, successive phases that were defined by the dominant process in each phase: growth on rbCOD, growth on slowly biodegradable COD (sbCOD), followed by decay. In this study typical values were assumed for all the kinetic and stoichiometric parameters, and hence the simulated respirometry responses were determined by the initial $\mathrm{Z}_{\mathrm{bh}}$, rbCOD and sbCOD concentrations. The active fraction was determined using the first approach described in the previous section. Musser (2009) then used linear regression to fit the modeled response to the measured respirometric data to yield estimates of rbCOD and sbCOD.

Similar to Musser (2009), Kianmehr (2010) analyzed the effects of sonication and ozonation on the aerobic biodegradability of WAS by employing respirometry to quantify the generation of rbCOD by pretreatment. In this study samples with low F/M ratios were employed such that the substrate was depleted quickly in the respirometry test. Kianmehr (2010) then developed an equation to estimate the concentration of rbCOD in the respirometry test. In this equation, the rbCOD was calculated on the basis of the oxygen consumed during the rapid uptake portion of the test less the oxygen uptake that was attributed to endogenous decay.

\subsection{Existing Pretreatment Models}

Sludge pretreatment modeling is currently in the early stages of development. A few research groups have proposed approaches for modeling WAS pretreatment technologies and an even fewer number have published stand-alone pretreatment models. Lei et al. (2010) integrated the ASM2d and ADM1 models into a proprietary whole-plant simulator that was employed to simulate a number of WWTPs employing sludge reduction technologies. At one plant, RAS was treated with ozone. The simulated data was fit to the measured data by testing a number of modifications to the ASM2d model that 
could characterize the ozone treatment such as a COD reduction due to oxidation and mineralization, conversion of $X_{i}$ to $X_{s p}$ and conversion of $Z_{b h}$ to $X_{s p}$ and $Z_{e}$. The results of this exercise were inconclusive because the sludge was not well characterized. At another plant, WAS was pretreated by HPTH. Lei et al. (2010) fit the simulations to the measured data by testing a number of modifications to ADM1 that were intended to characterize the impact of HPTH pretreatment such an increased digester hydrolysis rate and a partial conversion of inert decay products to carbohydrates, lipids and proteins. The results of this study were also inconclusive. Lei et al. (2010) recommended that further site-specific studies be carried out that focus on fractionating the COD in the sludge upstream and downstream of the sludge reduction technology.

Phothilangka et al. (2008) developed and calibrated a plant-wide model of the Zirl WWTP by integrating the ASM1 and ADM1 models in a Matlab/SIMBA simulation environment. At this plant, WAS was anaerobically digested then dewatered and the dewatering stream was recycled to the aeration basin. A continuous HPTH process was later implemented at the plant whereby WAS was pretreated at $180^{\circ} \mathrm{C}$ under 19 to 21 bars prior to anaerobic digestion. Similar to the approach taken by Lei et al. (2010), the original plant-wide model was adjusted to fit the data measured after pretreatment implementation by increasing the anaerobic disintegration rate and transforming inert decay products to biodegradable COD. The authors demonstrated that the measured ammonia increase after digestion due to the HPTH pretreatment could only be explained by a complete degradation of decay products. The measured COD concentration in the plant effluent increased after the HPTH process was implemented, indicating that the HPTH pretreatment process generated soluble non-biodegradable COD. The calibrated model successfully predicted this increase.

Dhar et al. (2012) monitored the performance of bench-scale anaerobic digesters that were fed with raw municipal WAS and thermo-chemical pretreated WAS respectively. The impact of pretreatment was simulated using the BioWin 3.0 Activated Sludge Digestion Model. Similar to the previously mentioned studies, these authors varied the digester hydrolysis rate to fit the simulations to the measured responses. Dhar et al. (2012) concluded that pretreatment increased the hydrolysis rate by $30 \%$ but did not enhance the digestibility of the sludge.

Frigon and Isazadeh (2010) and Musser (2009) developed COD-based pretreatment models that were rate dependent and could be integrated into whole-plant simulators. Frigon and Isazadeh (2010) developed and compared three hypotheses of the changes in COD fractionation that were caused by pretreating return activated sludge (RAS) with ozone. An extension of the IWA-ASM3 model was 
developed for each hypothesis and the model simulations were compared to the results of pilot-scale experiments and the findings of other researchers. The first model extension hypothesized that ozonation transformed biomass to substrate COD and non-biodegradable COD with a small fraction of the COD being oxidized. Storage products were similarly transformed except that nonbiodegradable COD was not generated from these materials. In addition to these transformations, the second model extension assumed that inert and substrate solids were also transformed. The third model extension contained the transformations of the second model extension and also included biomass inactivation processes. This model extension was found to provide the best fit of test data. The inactivation of biomass and associated release of stored COD was shown to occur at a higher rate than the transformation rate of the solids COD fractions. Frigon and Isazadeh (2010) found that the third model extension could be further improved by lowering the heterotrophic biomass level without further lowering the nitrifying biomass. This could be achieved by assuming lower inactivation rates for nitrifiers than for ordinary heterotrophs or assuming nitrifying biomass metabolically adapted to the ozone treatment which would have changed parameters such as the maximum specific growth rate.

Musser (2009) designed two separate COD-based stoichiometric pretreatment models, one for sonication and one for ozonation. These models were based on experiments using WAS from the New Hamburg WWTP in Ontario. Each pretreatment model was integrated into a WWTP simulation using BioWin version 3.0. This simulator did not permit a simple stoichiometric conversion of COD species hence a rate-based approach was required to implement the pretreatment model into the simulator. For each pretreatment model, Musser used the model builder reactor in BioWin. In order to model different pretreatment doses, Musser set the HRT of the pretreatment reactor using equation 2.5:

$$
H R T=e^{\frac{w}{k}}-1
$$

In equation $2.5, \mathrm{w}$ is the dose and $\mathrm{k}$ is the dose constant. For simplicity, Musser converted only one biomass fraction, the heterotrophic biomass, in both his pretreatment models.

During sonication, biomass was observed to be inactivated according to equation 2.6:

$$
\frac{Z_{b h}-Z_{b h 0}}{Z_{b h 0}}=e^{-\frac{w}{k}-1}
$$

In equation 2.6, $\mathrm{Z}_{\mathrm{bh}}$ and $\mathrm{Z}_{\mathrm{bh} 0}$ were the final and initial heterotroph concentrations. The experimental data showed that $45 \%$ of the inactivated biomass was converted to readily degradable substrate and 
$12 \%$ to slowly degradable substrate, which was assumed to be colloidal. Musser (2009) assumed that $8 \%$ of the inactivated biomass was cell residue and the remaining 35\% was converted to slowly degradable particulate COD. Musser's experiments also showed that $57 \%$ of slowly degradable COD was converted to readily degradable $\mathrm{COD}$ and the remainder was converted to colloidal biodegradable COD. This model therefore also included a separate process for this conversion. Each conversion proceeded at a rate dependent on the ultrasound dose. In Musser's study and the current study, colloidal matter was defined as that which passed through a filter with a pore size of $1.5 \mu \mathrm{m}$ but was retained by a filter with a pore size of $0.45 \mu \mathrm{m}$.

Musser (2009) designed a model for the ozonation pretreatment process based on experimental findings of COD solubilization, heterotroph inactivation and nitrate production. The ozone pretreatment data showed a rapid inactivation of heterotrophs and a much slower production of readily biodegradable COD (rbCOD); hence the process was modeled using two stages, both of which depended on the ozone dose. In the first stage, heterotrophs were converted to slowly biodegradable $\mathrm{COD}$ (sbCOD) and in the second stage the sbCOD was converted to rbCOD. These two stages were expressed by equations 2.7 and 2.8 :

(1) $Z_{b h}=Z_{b h 0} \times e^{-53 k}$

(2) $r b C O D=Z_{b h 0} \times 43.8 \%\left(1-e^{-18.3 k}\right)$

Similar to sonication, a cell residue fraction of $8 \%$ was assumed to remain after ozonation. Musser's ozone pretreatment model also included a process to convert nitrite to nitrate. Each conversion process depended on the ozone dose.

The simpler model proposed for sonication was based on the respirometric data whereas the more complex ozonation model was based on a combination of respirometric and nitrogen fraction data. In both models the extent of heterotroph conversion increased with the pretreatment dose however the rate and maximum conversion differed (Musser, 2009).

The sludge pretreatment modeling efforts discussed in this section were based on simulating real activated sludge systems before and after the application of a pretreatment technology. Various COD transformations associated with pretreatment have been proposed. However, these transformations were not solely empirical as the concentrations of some COD species were assumed. It is challenging to accurately fractionate the COD of real activated sludge because it is comprised of a complex mixture of components such as rbCOD, sbCOD, $\mathrm{S}_{\mathrm{us}}, \mathrm{X}_{\mathrm{i}}$, biomass and decay and storage products and some of these components are not readily isolated. For example, $\mathrm{X}_{\mathrm{i}}$ cannot be distinguished and 
separately measured from $Z_{e}$ when both are present in wastewater. Therefore a change in the measured concentration of non-biodegradable particulate COD could be due to a change in the concentration of $X_{\mathrm{i}}$ or $\mathrm{Z}_{\mathrm{e}}$ or both. Phothilangka et al. (2008) considered the transformation of $\mathrm{Z}_{\mathrm{e}}$ to bCOD but did not consider the transformation of $X_{i}$ to bCOD. Ramdani et al. (2010) have shown that the COD fractionation of activated sludge is substantially simplified when the system is fed with a synthetic soluble biodegradable substrate such as acetate. The MLSS composition of real and synthetically-fed activated sludge systems is depicted in Figure 2.1 (Ramdani et al., 2010).

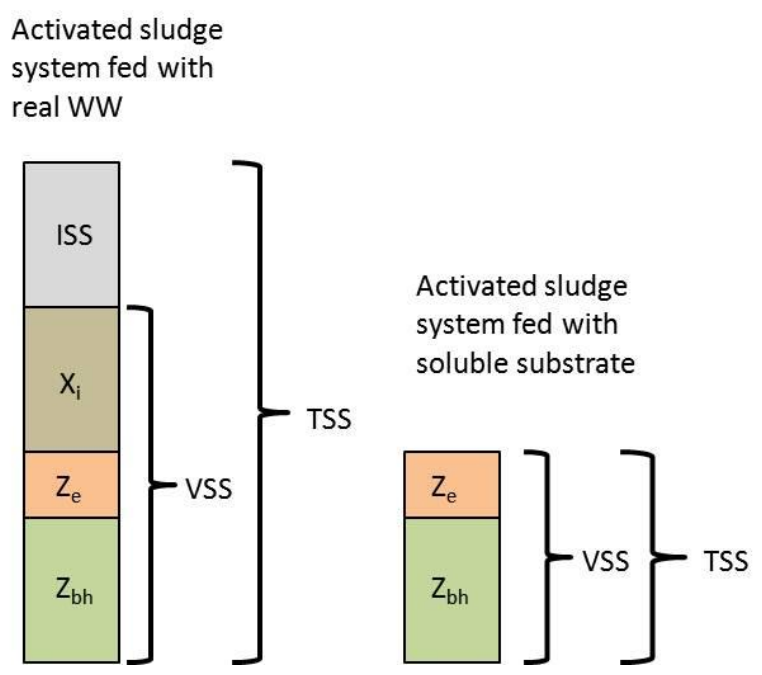

Figure 2.1 MLSS Composition of Activated Sludge Systems (Ramdani et al., 2010)

The COD concentrations of active biomass and endogenous decay products can be accurately measured using respirometric methods. In this way, WAS generated from a synthetically-fed biological reactor may be completely fractionated in terms of COD. Correctly characterizing the raw WAS would allow the fractionation of the pretreated WAS to be characterized more accurately. For example, any soluble COD present in the pretreated WAS would be a direct result of pretreatment since soluble COD would be absent in the raw WAS from a synthetically-fed biological reactor. In this project, WAS generated from a synthetically-fed biological reactor was fed to an aerobic digester for a period of time (Phase 1). The WAS was then pretreated prior to being fed to the aerobic digester and the system was operated for an additional period of time (Phase 2). The Phase 1 and 2 systems were each modeled and the models were calibrated using the measured COD fractions in the various WAS streams. The models were then used to estimate any missing COD fractions. In this way, it was possible to accurately determine the COD transformations caused by pretreatment.

The stand-alone pretreatment models proposed by Musser (2009) and Frigon and Isazadeh (2010) were rate-based. It is proposed that the pretreatment models may be simplified to stoichiometric 
COD transformations, without compromising the robustness of the simulations. The pretreatment models presented in this section were based on ozonation and sonication hence there is a need to develop an accurate model for the HPTH pretreatment process. 


\section{Materials and Methods}

\subsection{Reactor Design and Materials}

The overall process flow diagrams for the reactors used in this project are shown in Figure 3.1 and 3.2 .

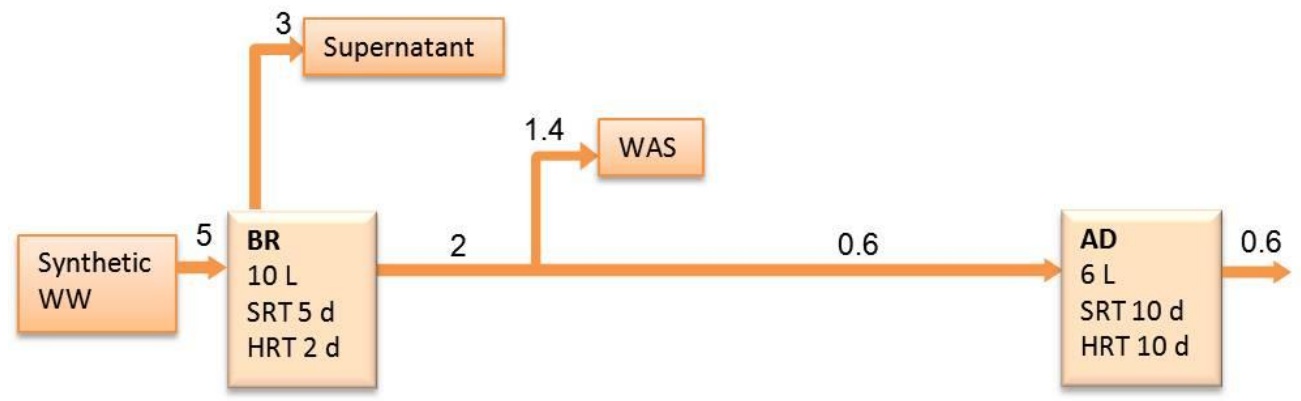

Figure 3.1 Phase 1 Process Flow Diagram (Flow in L/d)

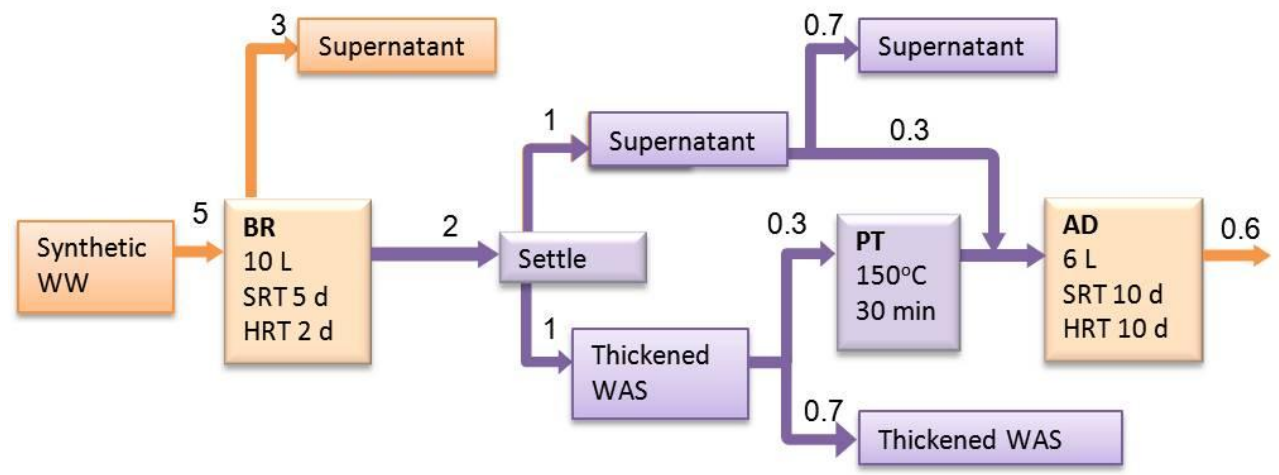

Figure 3.2 Phase 2 Process Flow Diagram (Flow in L/d)

A bench-scale synthetically fed biological reactor (BR) was used in this project to provide a source of stable WAS that consisted of active biomass and decay products. This WAS was used to start up and feed, on a daily basis, a bench-scale aerobic digester (AD). Once the AD had reached steady-state and was sufficiently characterized, a high temperature and pressure batch reactor was used to pretreat the BR WAS before it was fed to the AD.

Two identical transparent acrylic watertight containers were used for the bench-scale BR and AD reactors. These reactors were operated on a lab bench at a temperature of $20 \pm 1^{\circ} \mathrm{C}$ over the duration of the project. The containers were cylindrical with an internal diameter of $20 \mathrm{~cm}$, depth of $38 \mathrm{~cm}$ and total volume of $12 \mathrm{~L}$. Both reactors were fit with a transparent acrylic lid held in place by bolts. 
A hole was drilled into the center of each lid and fitted with a bearing. A stainless steel mixing shaft fitted with two propellers was inserted through this hole into the reactor. Mixing in each reactor was provided by a Bodine ${ }^{\circledR}$ Model 0158 DC Gearmotor that was connected to a power converter. Each mixer had a rotational speed of $200 \mathrm{rpm}$. A photograph of the biological reactor and aerobic digester are shown in Figure 3.3.

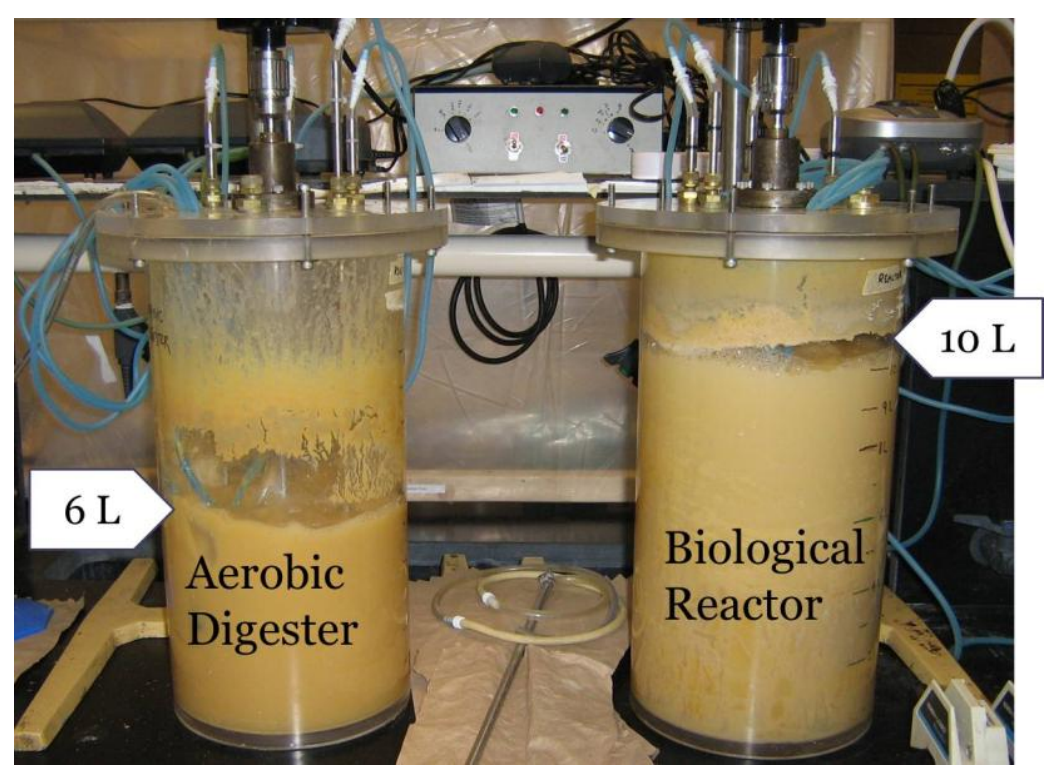

Figure 3.3 Biological Reactor and Aerobic Digester

Each reactor was provided with two TetraTec ${ }^{\circledR}$ Whisper AP300 Deep Water Air Pumps. In addition, the BR was equipped with two TopFin ${ }^{\circledR}$ AIR-3000 air pumps as well as the bench air supply. The air was directed from the pumps and bench air valve through Top Fin ${ }^{\circledR} 6 \mathrm{~mm}$ diameter silicone airline tubing. For the air supply from the TetraTec ${ }^{\circledR}$ pumps, the silicone tubes were connected to four 6 $\mathrm{mm}$ diameter stainless steel tubes inserted into holes in the lid of each reactor. The silicone tubing connected to the TopFin ${ }^{\circledR}$ pumps and bench air valve were inserted directly into the BR through a 3 $\mathrm{cm}$ diameter opening in the lid.

Liquid was removed from each reactor through a $6 \mathrm{~mm}$ stainless steel tube that was inserted into a hole in the lid. This tube was connected to flexible tubing and a peristaltic pump. Liquid was added to each reactor through a funnel inserted into a $3 \mathrm{~cm}$ diameter opening in the lid.

The high temperature and pressure pretreatment of WAS was performed using a Parr® Model 4563 Mini Pressure Reactor, shown in Figure 3.4. This reactor treated a maximum liquid volume of 400 
$\mathrm{mL}$ in batch mode. Continuous stirring was provided during operation by a variable speed motor. This reactor can operate up to a maximum pressure of 68 bars and a maximum temperature of $225^{\circ} \mathrm{C}$. Heating was provided by a mantle heater assembly connected to a programmable temperature control. Cooling was achieved by connecting the cold water tap on the lab bench to the cooling loop on the head of the reactor vessel using flexible tubing. The vessel was fit with a thermocouple that was connected to a display so the temperature of the reactor contents could be monitored.

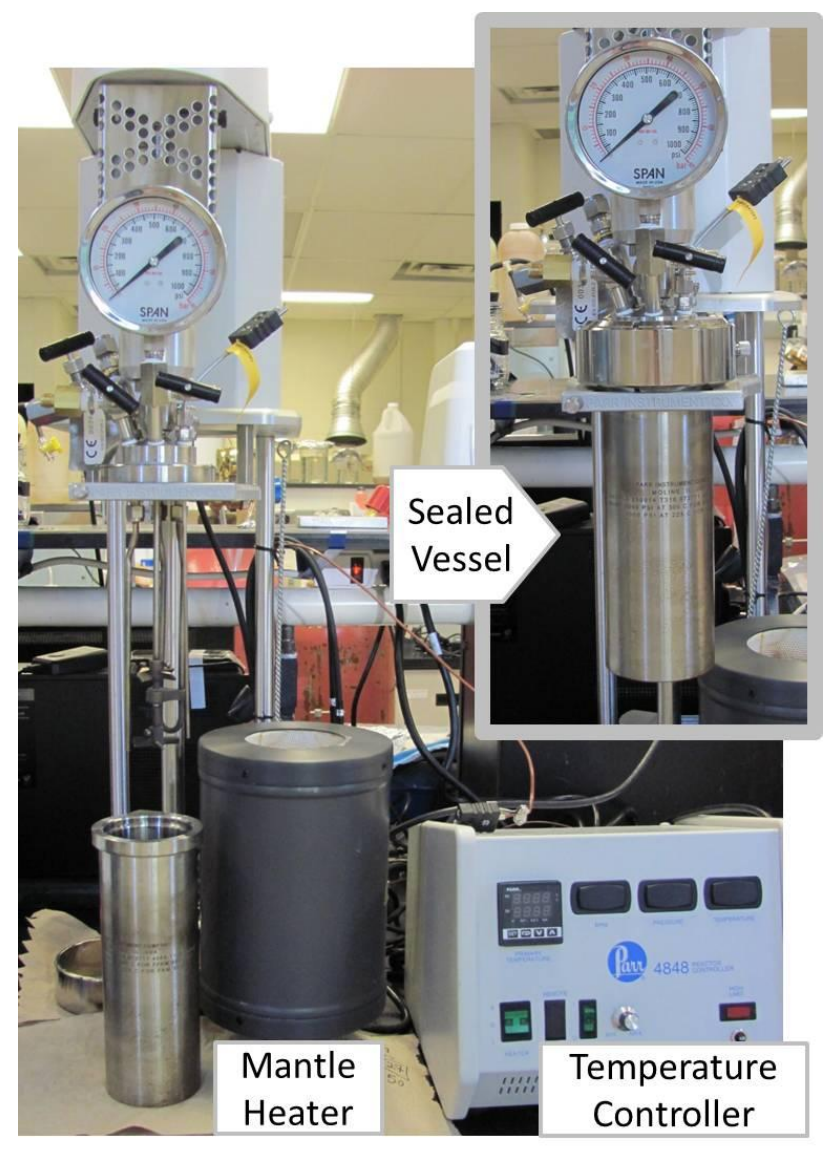

Figure 3.4 Parr ${ }^{\circledR}$ 4563 Mini Pressure Reactor for HPTH Pretreatment

\subsection{Synthetic Wastewater}

A synthetic wastewater was used to provide the organics, trace metals, nitrogen, phosphorous and alkalinity required for bacterial cell maintenance and growth in the BR. The selected synthetic wastewater recipe was adopted from Mohammadali and Hall (2011). This recipe was modified by increasing the concentration of every compound by a factor of 12.5 except sodium bicarbonate and ammonium chloride which were increased by factors of 1.5 and 6.5 respectively. This modification 
resulted in a theoretical total COD (TCOD) of the synthetic wastewater of 4,000 $\mathrm{mg}$ COD/L. It should be noted that from day 0 to 179 of the project, the synthetic wastewater concentrations were twice as high, i.e. theoretical TCOD of $8,000 \mathrm{mg}$ COD/L. The concentration was halved on day 179 , at the beginning of Phase 1, because it was found that the aeration requirements in the BR could not be satisfied with the higher strength feed. The minimum DO concentration in the reactor for uninhibited activity of aerobic heterotrophs is $2 \mathrm{mg} \mathrm{O}_{2} / \mathrm{L}$. The synthetic feed was prepared in stock solutions every 6 to 12 days and stored in the fridge. The inorganic chemicals (Table 3.2) were kept in a separate solution from the organic chemicals (Table 3.1) to prohibit the growth of microorganisms in the stock solution that would have depleted the COD of the organic feed. The yeast extract is listed with the organics to show its contribution to the TCOD however it was included with the inorganic stock solution because it also contained inorganic nutrients. The compound concentrations in the synthetic wastewater fed to the BR are summarized in Tables 3.1 and 3.2.

Table 3.1 Organic Synthetic Wastewater

\begin{tabular}{|l|c|c|}
\hline Organic Synthetic Feed & COD Fraction & Concentration (mg/L) \\
\hline yeast extract & 0.12 & 350 \\
\hline glucose $\left(\mathrm{C}_{6} \mathrm{H}_{12} \mathrm{O}_{6}\right)$ & 0.14 & 525 \\
\hline starch $\left(\mathrm{C}_{6} \mathrm{H}_{10} \mathrm{O}_{5}\right)_{n}$ & 0.23 & 775 \\
\hline sodium acetate $\left(\mathrm{NaCH}_{3} \mathrm{COO}\right)$ & 0.26 & 1315 \\
\hline acetic acid $\left(\mathrm{C}_{2} \mathrm{H}_{4} \mathrm{O}_{2}\right)$ & 0.25 & 1100 \\
\cline { 1 - 3 } Column Total $=$ & 1 & \multicolumn{1}{|c}{} \\
\cline { 2 - 3 } & \multicolumn{2}{|c|}{}
\end{tabular}

Table 3.2 Inorganic Synthetic Wastewater

\begin{tabular}{|l|c|}
\hline Inorganic Synthetic Feed & Concentration $(\mathbf{m g} / \mathbf{L})$ \\
\hline monopotassium phosphate $\left(\mathrm{KH}_{2} \mathrm{PO}_{4}\right)$ & 265 \\
\hline sodium bicarbonate $\left(\mathrm{NaHCO}_{3}\right)$ & 167 \\
\hline ammonium chloride $\left(\mathrm{NH}_{4} \mathrm{Cl}\right)$ & 665 \\
\hline magnesium chloride $\left(\mathrm{MgCl}_{2} * 6 \mathrm{H}_{2} 0\right)$ & 300 \\
\hline calcium chloride $\left(\mathrm{CaCl}_{2} * 2 \mathrm{H}_{2} 0\right)$ & 450 \\
\hline zinc sulphate $\left(\mathrm{ZnSO}_{4} * 7 \mathrm{H}_{2} \mathrm{O}\right)$ & 5 \\
\hline
\end{tabular}

In order to enhance the flocculation process in the $\mathrm{BR}$, a ferric chloride solution was added to the $\mathrm{BR}$ at the same time as the synthetic feed. The influent concentration was $125 \mathrm{mg} / \mathrm{L} \mathrm{FeCl}_{3} * 6 \mathrm{H}_{2} \mathrm{O}$. This was made using a stock solution of $17.54 \mathrm{~g} / \mathrm{L} \mathrm{FeCl}_{3} * 6 \mathrm{H}_{2} \mathrm{O}$ that was prepared every 28 days.

Measurements of the COD, SS and $\mathrm{pH}$ of the synthetic feed were taken at least once per month throughout the project to monitor any fluctuations in the feed concentrations. Both the fresh and two 
week old stock solutions were measured and it was found that the COD did not change significantly during two weeks of storage in the fridge. The COD fractionation of the synthetic feed will be presented in section 4.2.1.

\subsection{Operation of Reactors}

\subsubsection{Biological Reactor}

The BR was operated from startup until the end of the project on day 283. This reactor was operated as an SBR as shown in Figure 3.5. At start-up, 5 liters of AS were collected from the aerated biological reactor at the Waterloo WWTP and added to the bench-top BR. This provided the BR with a diverse spectrum of microorganisms. The reactor was mixed continuously and aeration was provided such that the DO concentration remained above $2 \mathrm{mg} / \mathrm{L}$. After 5 hours, one liter of synthetic feed solution was added to the BR every hour until the total volume in the BR reached $10 \mathrm{~L}$.

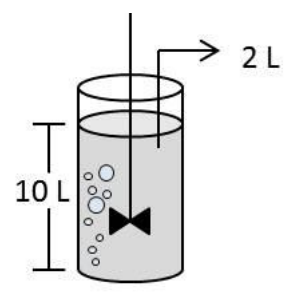

1. Withdraw WAS

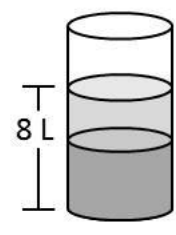

2. Settle

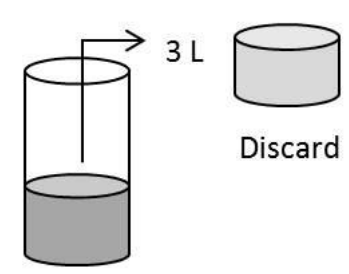

3. Decant supernatant

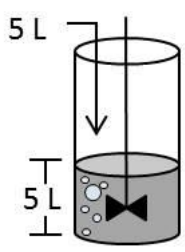

4. Add synthetic feed

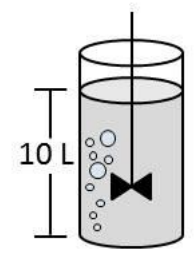

5. React ( $22 \mathrm{~h}$ )

\section{Figure 3.5 Daily Operation of the Biological Reactor}

From Figure 3.5 it can be seen that WAS was removed from the BR while the reactor was being mixed and aerated. Mixing and aeration were then ceased to allow the contents to settle. Once the sludge blanket settled below the $5 \mathrm{~L}$ graduation mark on the reactor, $3 \mathrm{~L}$ of supernatant was siphoned off. Mixing and aeration were then resumed and $5 \mathrm{~L}$ of synthetic feed was added to the reactor. The 
DO concentration in the reactor dropped to almost zero immediately after feeding however it was ensured that the DO concentration rose above $2 \mathrm{ppm}$ within two hours after feeding and remained at that level for the remainder of the reaction period. Steps 1 through 4 in Figure 3.5 typically required a total of 2 hours leaving approximately 22 hours for the reaction period. These steps were repeated daily, 7 days per week. The hydraulic residence time (HRT) of the BR was $2 \mathrm{~d}$ and the SRT was $5 \mathrm{~d}$.

The BR was cleaned twice per week throughout its operation. This was necessary to remove any biofilm that collected on the inside walls and lid of the reactor as well as on the propeller blades and steel tubes inside the reactor. The reactor was cleaned after the supernatant had been removed since this was the time when the reactor contained the smallest volume $(5 \mathrm{~L})$. The mixer and aerators were turned off and the lid was opened. Tissues were used to wipe off the sludge collected on the inside lid and walls above the liquid level. The propeller, shaft and steel tubes were removed from the liquid and wiped clean using tissues. A clean handheld brush was used to scrape the biofilm from the walls below the liquid level. During the third week of operation of the reactor, worms became visible on the inside walls and lid. These worms were removed by hand and did not appear again.

\subsubsection{Aerobic Digester Fed with Raw BR WAS}

The aerobic digester was started up with BR WAS on day 20 and operated until the end of the project, day 283. As will be shown in section 4.1, the BR was predicted to reach steady state conditions by day 15 hence it was assumed that the AD was started up with a stable WAS source. On day 20, $2 \mathrm{~L}$ of WAS from the BR was poured into the $\mathrm{AD}$ and the mixer and aerators were turned on. This was repeated for the following two days so that the AD contained $6 \mathrm{~L}$ of AS on day 22. From day 23 until day 77, the AD was operated as a SBR with an HRT of $3 \mathrm{~d}$ and SRT of $10 \mathrm{~d}$. The BR-AD system was modeled in BioWin 3.1 and it was predicted that the AD reached steady state conditions within 3 SRTs or 30 days from the day it was started up. On day 77, the operation of the AD was simplified so that the HRT and SRT were both $10 \mathrm{~d}$, as depicted in Figure 3.6. This operation was continued until the end of the project. 


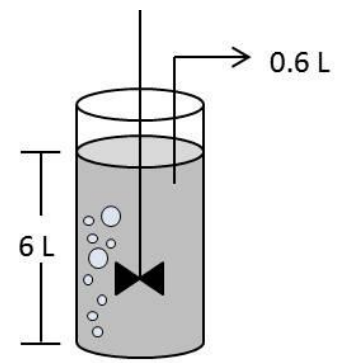

1. Withdraw WAS

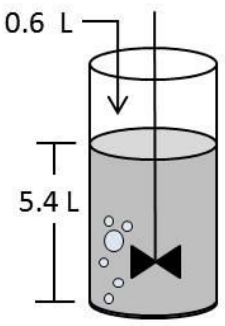

2. Add BR WAS

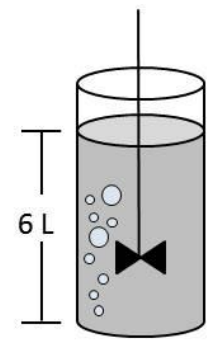

3. React ( $22 \mathrm{~h})$

Figure 3.6 Daily Operation of the Aerobic Digester (day 77 to 283)

The main reason for changing the operation of the AD was to decrease the TCOD of the AD WAS so that the aeration requirements in the $\mathrm{AD}$ could be satisfied. As mentioned in section 3.2, the minimum DO concentration in the reactor for the uninhibited activity of aerobic heterotrophs is $2 \mathrm{mg}$ $\mathrm{O}_{2} / \mathrm{L}$. The second reason was to make available a portion of the BR WAS for running tests. The entire daily volume of BR WAS was fed to the AD when the AD was operated as a SBR. In the modified operating regime, only $30 \%$ of the daily BR WAS volume was fed to the AD. This change in the operation of the $\mathrm{AD}$ was simulated using BioWin 3.1 and it was predicted that the $\mathrm{AD}$ reached steady state conditions after 3 SRTs, on day 107.

Similar to the BR, the AD was cleaned twice per week throughout its operation. The digester was cleaned when it contained the smallest volume of mixed liquor. The $\mathrm{AD}$ was cleaned in the same way as the BR. Unlike the BR, worms did not appear at any time during the operation of the AD.

\subsubsection{Aerobic Digester Fed with Pretreated BR WAS}

On day 203, pretreatment of the BR WAS that was fed to the AD was initiated on a daily basis. The pretreatment reactor was always operated at its maximum capacity of $400 \mathrm{~mL}$. The mantle heater and programmable temperature controller were used to increase the temperature of the sludge to $150^{\circ} \mathrm{C}$. This took approximately 20 minutes. The Parr® 4563 reactor is a pressure vessel designed to prevent any gas or liquid from escaping. At $150^{\circ} \mathrm{C}$, the internal pressure was 3 bars. No additional pressure was applied to the vessel. The temperature was held at $150^{\circ} \mathrm{C}$ for 30 minutes. The mantle heater was then turned off and removed from the reaction vessel and the cold water tap connected to the cooling coil was turned on. It took approximately 45 minutes to decrease the internal temperature from $150^{\circ} \mathrm{C}$ to $20^{\circ} \mathrm{C}$. 
Once empty, the inside of the pressure vessel was washed clean in the sink using a brush with soap and water. The lid and attached mixing shaft, cooling coil and sampling ports were also cleaned. These components were carefully inspected to insure no sludge remained before the next pretreatment batch was started.

The AD required a daily feed of $600 \mathrm{~mL}$. In addition, up to $1 \mathrm{~L}$ of pretreated WAS was required to run tests. To meet these volume requirements, the $2 \mathrm{~L}$ of WAS that was withdrawn from the BR was allowed to settle in a $2 \mathrm{~L}$ graduated cylinder until the sludge blanket level was below the $1 \mathrm{~L}$ graduation mark. The supernatant was then siphoned off and retained. The remaining $1 \mathrm{~L}$ of thickened sludge was then stirred and $400 \mathrm{~mL}$ was poured into the pretreatment reactor. Following pretreatment, $300 \mathrm{~mL}$ of pretreated sludge was then diluted to $600 \mathrm{~mL}$ using the BR supernatant that had been removed from the graduated cylinder. This sludge was then fed to the AD. This sequence is illustrated in Figure 3.7.

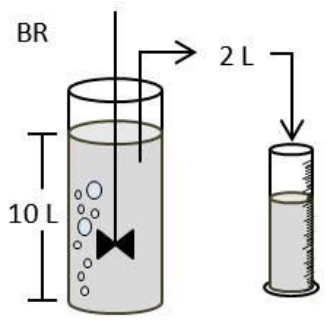

1. Withdraw WAS from BR

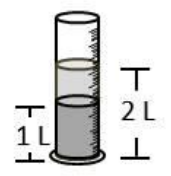

2. Let WAS settle

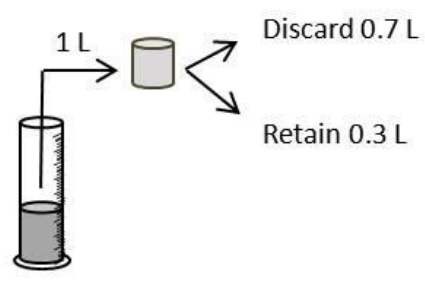

3. Withdraw supernatant

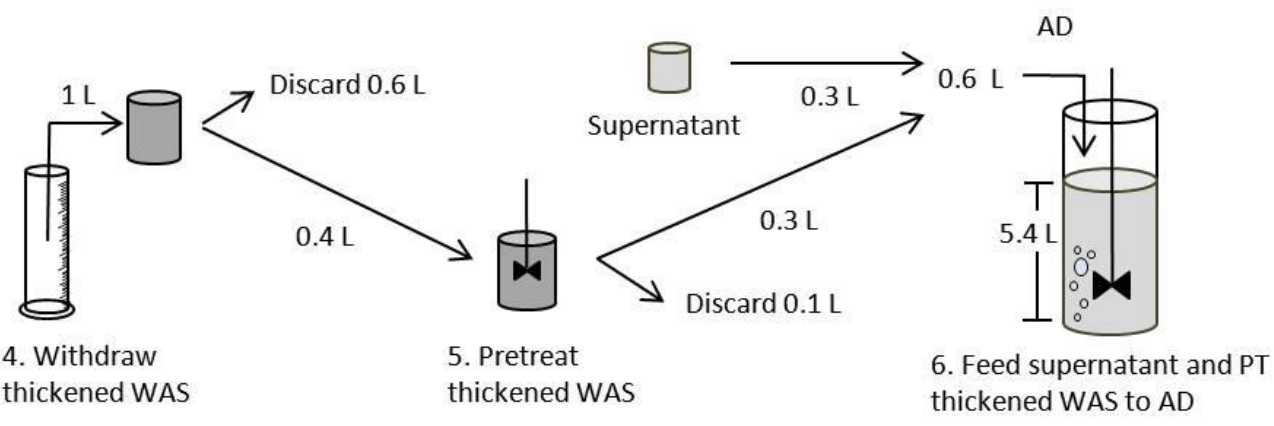

Figure 3.7 Daily Pretreatment Procedure (day 203 to 283)

Under this operation, $100 \mathrm{~mL}$ of pretreated thickened sludge and $700 \mathrm{~mL}$ of BR supernatant were available daily for running tests. There was also an excess volume of $600 \mathrm{~mL}$ of BR WAS that had 
been thickened in the graduated cylinder. On days when greater volumes of pretreated WAS were required for running tests, a second batch of thickened BR WAS was pretreated.

\subsection{Project Timeline and Sampling Schedule}

An overview of the monitoring and modeling conducted to address the objectives of the project is presented in Figure 3.8.

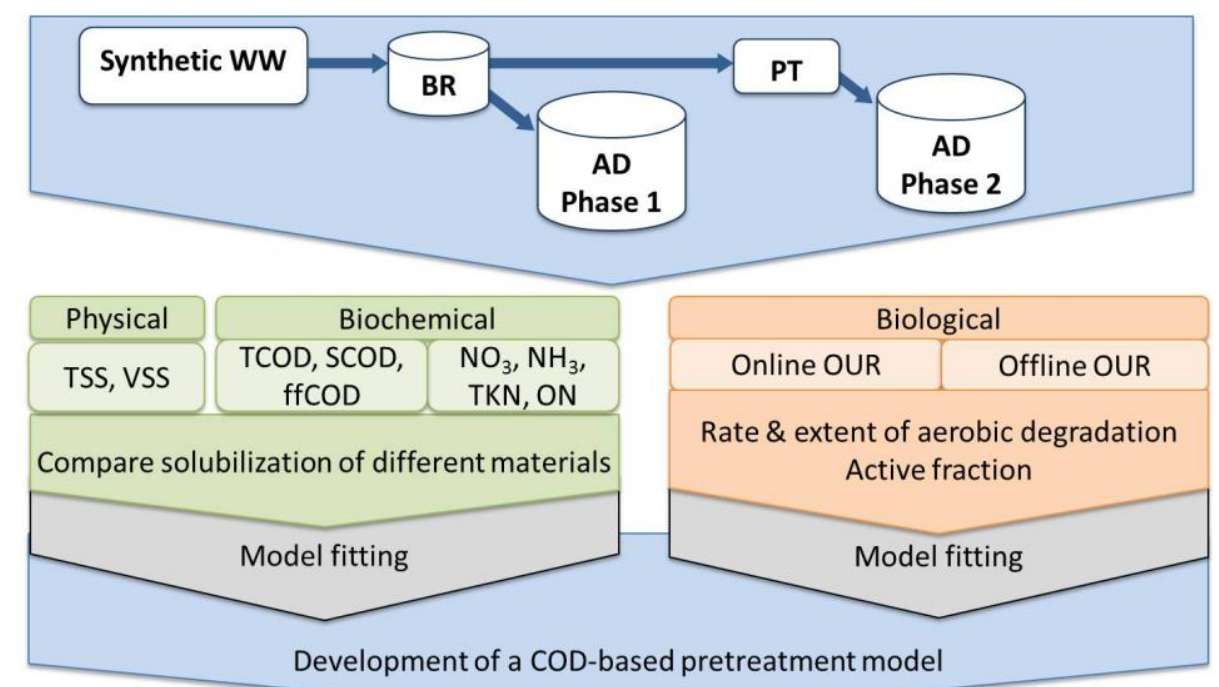

Figure 3.8 Overall Framework for Characterization of Impacts of HPTH Pretreatment on WAS

The project timeline is shown in Figure 3.9. The focus of the project is the period from day 179 to 283, when Phases 1 and 2 took place. 


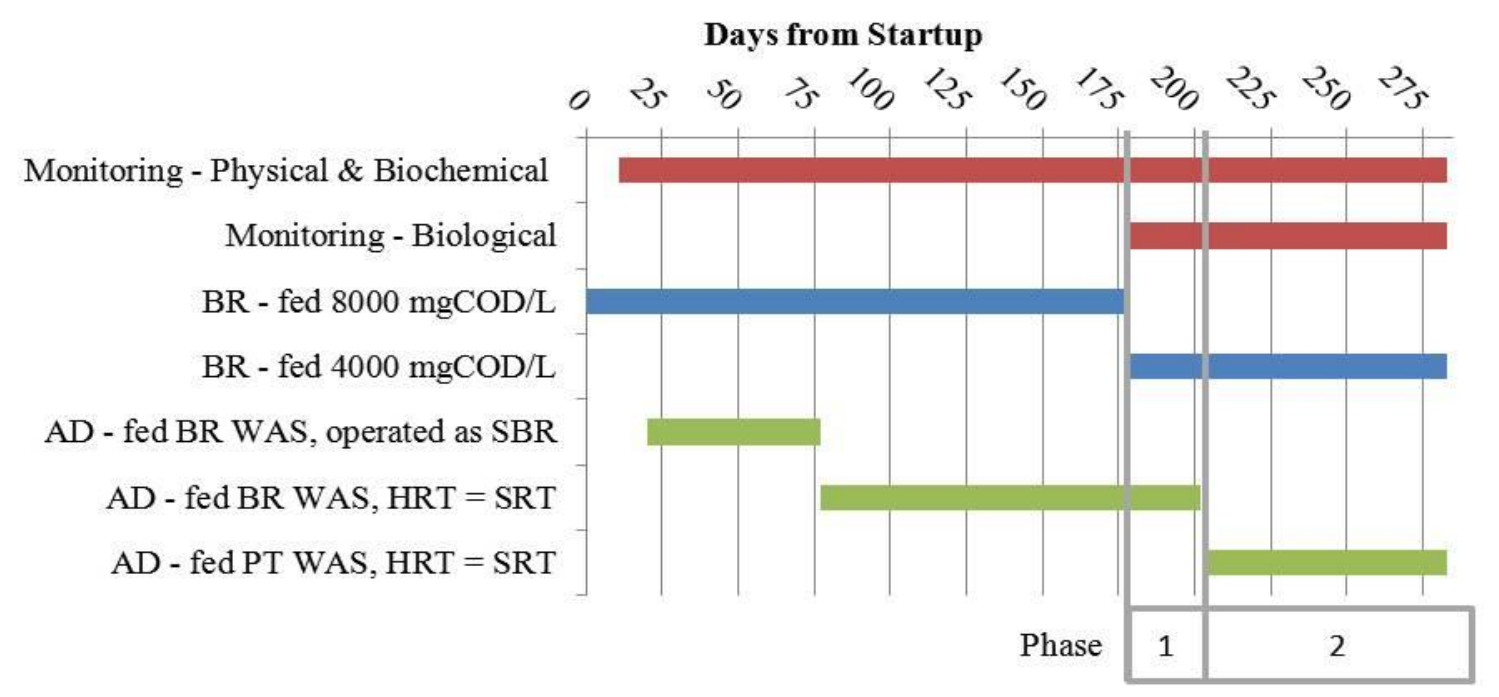

Figure 3.9 Project Timeline

When needed for characterization, samples of AS were taken from the BR and AD at the end of the reaction period, approximately 22 hours after the reactors had been fed. While the reactors were being mixed and aerated, samples were withdrawn by inserting the sampling tube well below the liquid level and at least $5 \mathrm{~cm}$ away from the floor and walls of the reactor. The sampling schedule is presented in Table 3.3.

Table 3.3 Sampling Schedule for Physical and Biochemical Tests

\begin{tabular}{|l|l|l|l|l|l|l|l|}
\hline Period & pH & $\begin{array}{l}\text { TCOD \& } \\
\text { SCOD }\end{array}$ & ffCOD & $\begin{array}{l}\text { TSS \& } \\
\text { VSS }\end{array}$ & NO3 & NH3 & $\begin{array}{l}\text { STKN \& } \\
\text { sON }\end{array}$ \\
\hline WAS from BR \\
\hline Day 0 to 20 & Daily & Once & & Once & & & \\
\hline WAS from AD and BR \\
\hline Day 20 to 92 & Daily & Bimonthly & Monthly & Bimonthly & Monthly & & \\
\hline WAS from AD, Pretreated and Raw WAS from BR \\
\hline Day 92 to 112 & Daily & Weekly & Weekly & Weekly & & & \\
\hline Day 112 to 134 & Daily & Weekly & Weekly & Weekly & Weekly & Weekly & \\
\hline Day 112 to 160 & Daily & & & & & & \\
\hline Day 160 to 236 & Daily & Weekly & Weekly & Weekly & Weekly & Weekly & \\
\hline Day 236 to 283 & Daily & Weekly & Weekly & Weekly & Weekly & Weekly & Weekly \\
\hline
\end{tabular}

Two types of biological analyses were carried out on the activated sludge. Offline respirometric tests were performed using a Challenge ${ }^{\circledR}$ AER-208 Respirometer. The test was run until the endogenous OUR curve was clearly detectable which was typically between 100 to 160 hours. Online respirometric tests were conducted by allowing the DO in the reactor to fluctuate by automatic 
aeration control and then measuring the rate of decline of DO. The online respirometric tests were conducted over three consecutive days. The purpose of this measurement was to determine the OUR in the reactor throughout the 22 hour reaction period. The offline respirometric tests were conducted bimonthly from day 83 to 173 and weekly from day 173 to 283 . The online respirometric tests were conducted monthly from day 173 to 204 and weekly from day 204 to 283.

\subsection{Analytical Methods}

The physical and biochemical indicators listed in Figure 3.8 were determined by conventional analyses. These analyses were conducted according to the relevant sections of Standard Methods for the Examination of Water and Wastewater (Eaton et al., 2005). Every sample was measured in duplicate. Blank and standard samples were prepared and measured for all of the biochemical tests.

\subsubsection{Suspended Solids}

A volume of $5 \mathrm{~mL}$ was filtered through a prepared $1.5 \mu \mathrm{m}$ pore size filter. The residue and paper were dried to a constant weight in an oven at $105^{\circ} \mathrm{C}$. The increase in weight of the filter represented the total suspended solids (TSS). The residue and paper were then placed in a furnace at $550^{\circ} \mathrm{C}$ for 45 minutes. The remaining solids represented the inorganic suspended solids (ISS) and the weight lost on ignition was the volatile suspended solids (VSS).

\subsubsection{COD}

The total, soluble (sCOD) and flocculated and filtered COD (ffCOD) were measured at $600 \mathrm{~nm}$ using a HACH DR/2000 Spectrophotometer. A range of standard samples were prepared and measured to generate a calibration curve. For the total COD (TCOD) analysis, the samples were first homogenized for 30 seconds then diluted by an appropriate factor. A volume of $2.5 \mathrm{~mL}$ of the emulsified, diluted sample was then added to the COD vial containing the reagents. The vial was then mixed by being inverted several times and then placed in the preheated HACH COD Reactor for 3 hours at $150^{\circ} \mathrm{C}$. 
For the soluble COD (sCOD) analysis, $50 \mathrm{~mL}$ of the sample was centrifuged for 30 minutes. The supernatant was then filtered through a Whatman Glass Microfibre filter (934-AH) with a pore size of $1.5 \mu \mathrm{m}$. The filtrate was then diluted if necessary, added to the COD vial and heated in the COD reactor identically to the TCOD sample. It should be noted that both soluble and colloidal matter were measured in this SCOD procedure. In this study, colloidal matter was defined as that which passed through a filter with a pore size of $1.5 \mu \mathrm{m}$ but was retained by a filter with a pore size of 0.45 $\mu \mathrm{m}$.

The flocculated and filtered COD (ffCOD) analysis is designed to measure the truly soluble COD. The sample was taken from the filtrate that had been collected for the SCOD sample. A stock solution of $25 \mathrm{~g} / \mathrm{L}$ of alum was prepared. Using this stock solution, $2.5 \mathrm{mg}$ of alum was added to a 50 $\mathrm{mL}$ of the sample filtrate. The sample was mixed vigorously for 30 seconds to begin flocculation, allowed to stand for 10 minutes, and then centrifuged for 15 minutes. Finally the sample was filtered using a $0.45 \mu \mathrm{m}$ pore size filter. The filtrate was diluted if necessary, added to a COD vial, mixed and then heated in an identical manner to the TCOD and SCOD samples. As mentioned, blank and standard samples were also subjected to this procedure. This made it possible to detect whether the COD was significantly altered by the ffCOD procedure.

\subsubsection{Ammonia}

The concentration of ammonia in samples was measured using a Thermo Scientific Orion 9512HPBNWP High Performance Ammonia Electrode. The sample was taken from the filtrate that had been collected for the sCOD sample. The ammonia probe was inserted into the sample while it was mixed continuously. The $\mathrm{pH}$ of the sample was then raised to above 11 using a $5 \mathrm{M} \mathrm{NaOH}$ solution. The ammonia reading was taken once the ammonia concentration had stabilized. A calibration curve was prepared using ammonia standards every time a set of samples was measured.

\subsubsection{Total Kjeldahl Nitrogen}

Both total Kjeldahl nitrogen (TKN) and soluble TKN (sTKN) concentrations were measured. Samples analyzed for TKN measurements were first homogenized for 30 seconds. The soluble 
sample was taken from the filtrate that had been collected for SCOD analysis. The digestion solution was prepared by dissolving $40 \mathrm{~g}$ potassium sulfate and $2 \mathrm{~mL}$ selenium oxychoride in $250 \mathrm{~mL}$ concentrated sulfuric acid and then diluting the solution to $500 \mathrm{~mL}$ with deionized water. A volume of $1 \mathrm{~mL}$ of sample and $1.5 \mathrm{~mL}$ of digestion solution were added to a TKN digestion tube. The tube was heated uncovered in a Bran \& Lubbe BD-40 block digester first at $220^{\circ} \mathrm{C}$ for $1.5 \mathrm{~h}$ and then at $380{ }^{\circ} \mathrm{C}$ for $2.5 \mathrm{~h}$ in order to convert organic nitrogen to ammonia. This method was developed in the Environment Canada Wastewater Technology Center in Burlington, Ontario. The ammonia was measured using the ammonia probe following the procedure described previously.

\subsubsection{Organic Nitrogen}

The organic nitrogen $(\mathrm{ON})$ was estimated by subtracting the ammonia concentration from the TKN concentration for a particular sample. Both the total $\mathrm{ON}$ and soluble $\mathrm{ON}(\mathrm{sON})$ concentrations were calculated.

\subsubsection{Nitrate}

Nitrate was measured in the soluble sample taken from the filtrate that had been collected for sCOD analysis. The sample was first diluted if necessary and then $25 \mathrm{~mL}$ of sample was poured into a sample cell and one HACH NitraVer® 5 Nitrate Reagent powder pillow was added. Once sufficient time had elapsed for the cadmium reduction to complete, the sample was analyzed at $400 \mathrm{~nm}$ using the HACH DR/2000 Spectrophotometer.

\subsection{7 pH}

The $\mathrm{pH}$ of the pretreated sludge and the activated sludge in the $\mathrm{BR}$ and $\mathrm{AD}$ was measured daily using an Omega PHB-600R pH Benchtop Meter. 


\subsection{Respirometric Methods}

Two respirometric methods were used in this project: online and offline. Both methods were used to determine the oxygen uptake rate (OUR) and cumulative oxygen uptake ( $\Sigma \mathrm{OU})$ associated with the WAS from the BR. The oxygen uptake data from both respirometric methods was used to assess the impact of pretreatment on the aerobic degradability of the WAS. In addition, the oxygen uptake data from the offline respirometric test was used to determine the activity of the microorganisms in the WAS. The overall goal of the respirometry measurements was to assist in fractionating the COD of the raw and pretreated WAS samples and hence only the carbonaceous oxygen demand was desired. The nitrogenous oxygen demand was inhibited by adding HACH 2533 nitrification inhibitor to the WAS. The collection and analysis of respirometric data is not standardized hence the two approaches are described here in detail.

\subsubsection{Online Respirometry}

Online respirometry was used to measure the OUR and $\Sigma \mathrm{OU}$ in the aerobic digester over the reaction period. Throughout the project, the temperature in the aerobic digester was $20 \pm 1^{\circ} \mathrm{C}$ and the $\mathrm{pH}$ was $8.3 \pm 0.2$. A Jenco@ model LD-900-5-DO Industrial Inline DO Probe was used to continuously measure the DO concentration in the reactor. This probe and the digester aerators were connected to a Jenco $\odot$ model 6309-PDT Advanced Multi-Parameter Analyzer. The probe, analyzer, aerators and $\mathrm{AD}$ are shown in Figure 3.10. The analyzer was programmed to turn on the aerators when the DO concentration in the reactor declined to $3 \mathrm{mg} \mathrm{O} / \mathrm{L}$ and turn off the aerators when the concentration

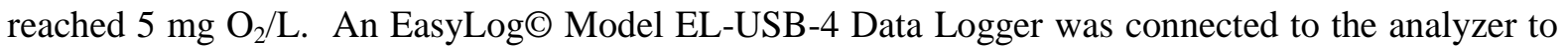
record the DO concentration in the reactor every 10 seconds. This logger could store up to 3.7 days of data; hence each online respirometry test was run for 3 days. The rate of DO decline was calculated for every time period between the high and low DO set points to generate OUR values. 


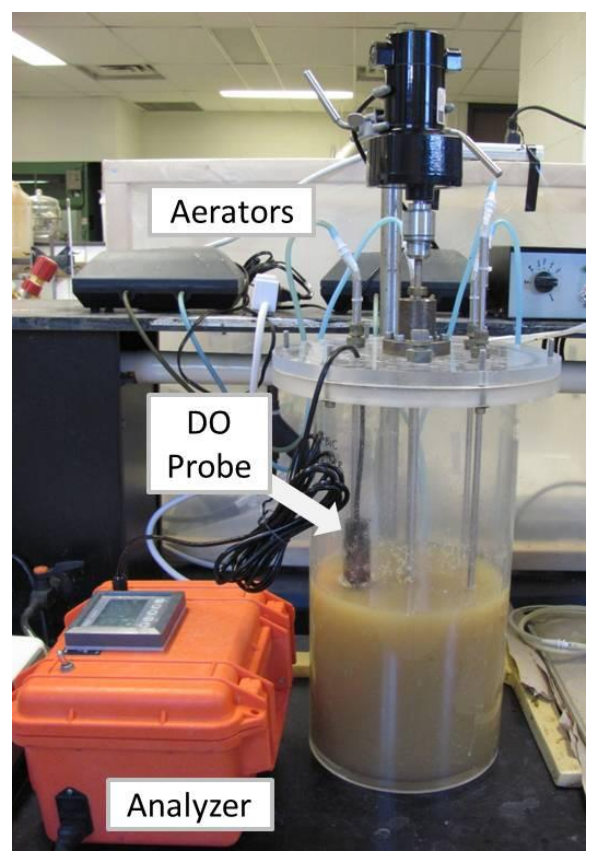

Figure 3.10 DO Probe and Analyzer Connected to Aerators in the AD

The nitrate levels in the aerobic digester were routinely measured as described in the previous section. Two grams of HACH 2533 nitrification inhibitor were added to the digester every two weeks to keep the nitrate level below $0.5 \mathrm{mg} \mathrm{N} / \mathrm{L}$. This essentially eliminated the nitrogenous oxygen demand in the digester.

\subsubsection{Offline Respirometry}

Offline respirometry was performed using a Challenge Technology@ AER-208 Respirometer. The temperature was set at $25^{\circ} \mathrm{C}$ for all respirometry tests and the $\mathrm{pH}$ was in the range of $8.1 \pm 0.5$. This direct input respirometer replenished the oxygen consumed by the microorganisms in the sample by injecting oxygen bubbles into the sample headspace using fluid action (Young and Cowan, 2004). The pressure drop caused by the consumption of oxygen initiated the injection of the oxygen bubbles. Each flow cell was carefully calibrated to determine the mass of oxygen in one bubble. The bubbles were counted by the respirometer and the $\Sigma \mathrm{OU}$ in each sample was recorded every 10 minutes. The change in oxygen consumption for each time step was calculated to provide an estimate of the OUR in the sample bottle throughout the test. Each offline respirometry test was run until a distinct decay 
curve was observed, indicating that most of the aerobically biodegradable material had been consumed. The test duration was typically 100 to 160 hours.

The carbon dioxide gas produced by the aerobic heterotrophs was removed from the headspace by suspending a vial containing $30 \% \mathrm{w} / \mathrm{w}$ potassium hydroxide in the sample vessel. In order to ensure that heterotrophic processes dominated all responses, $300 \mathrm{mg}$ of $\mathrm{HACH} 2533$ Nitrification Inhibitor was added to each sample bottle. The total volume of each sample bottle was $250 \mathrm{~mL}$ and a maximum of eight sample bottles could be tested simultaneously. The contents of the sample bottles were mixed using magnetic stir bars and the stirring rate was adjusted to produce a vortex that touched the stir bar. The sample bottles were contained in a water bath so that the temperature could be held constant at $25^{\circ} \mathrm{C}$. The sample bottles, water bath, oxygen flow measurement cells and oxygen supply manifold of the respirometer are pictured in Figure 3.11. The contents of the sample bottles for the offline respirometric tests are summarized in Table 3.4.
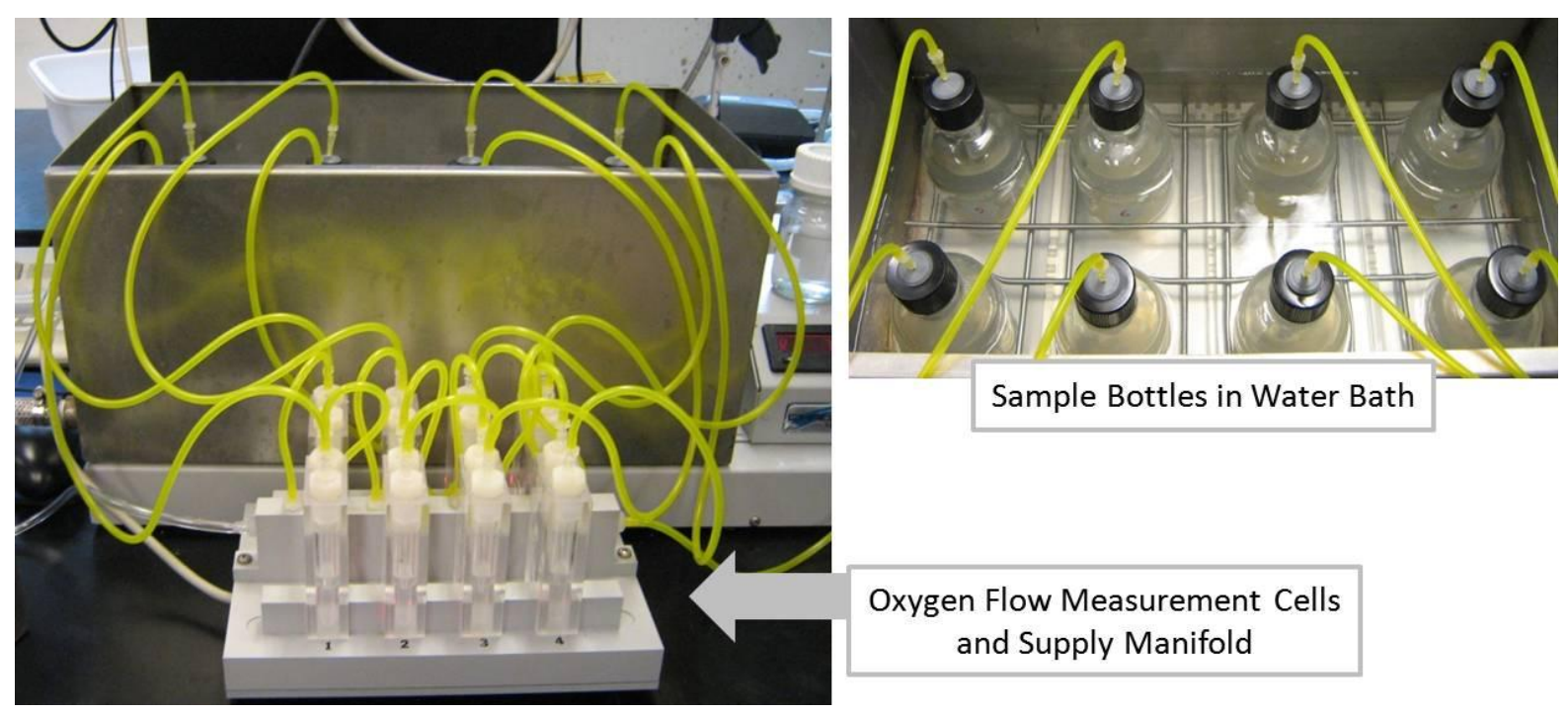

Figure 3.11 Challenge Technology AER-208 Respirometer

Table 3.4 Sample Bottle Contents for Offline Respirometric Tests

\begin{tabular}{|c|c|c|c|}
\hline Sample Bottle & Whole Substrate & Filtered Substrate & Inoculum \\
\hline 1,2 & $150 \mathrm{~mL}$ & & $50 \mathrm{~mL}$ \\
\hline 3,4 & & & $50 \mathrm{~mL}$ \\
\hline 5,6 & $150 \mathrm{~mL}$ & & \\
\hline 7,8 & & $150 \mathrm{~mL}$ & $50 \mathrm{~mL}$ \\
\hline
\end{tabular}


As shown in Table 3.4, every sample was tested in duplicate. The WAS samples used in offline respirometry were taken from the reactors at the end of the reaction period. In Table 3.4, the substrate was BR WAS in Phase 1 and pretreated WAS in Phase 2. The filtered substrate was prepared in the same way that samples were prepared for the SCOD test, i.e. centrifuged then filtered through a 1.5 $\mu \mathrm{m}$ glass fiber filter. In Table 3.4, the inoculum was the AD WAS. The F/M ratio in the inoculated bottles was 5 to 10 which was high enough to allow clear identification of the growth OUR response if present in the sample bottle.

Once a month during Phase 2, offline respirometry was carried out on sample bottles containing only $150 \mathrm{~mL}$ of BR WAS to verify that the active heterotroph concentration remained constant throughout the project. On day 278, an alternative offline respirometric test was used to measure the active fraction of the BR WAS. In this test, the sample bottles contained BR WAS and synthetic feed to yield F/M ratios in the range of 10 to 40 . The four pairs of bottles contained $0.5,1,2$ and $4 \mathrm{~mL}$ of BR WAS, respectively, each topped up to $200 \mathrm{~mL}$ with synthetic feed. Thus the BR WAS was diluted 400, 200, 100 and 50 times, respectively, in the four pairs of bottles. Respirometry carried out on control bottles consisting of water or synthetic feed showed zero oxygen uptake hence it was concluded that the observed oxygen uptake in the sample bottles was due to the metabolism of the microorganisms. 


\section{Results}

\subsection{Startup of Reactors}

At the beginning of the project, the operation of the BR was simulated using the BioWin 3.1 Integrated Model to predict the changes in concentration of the various COD components during the startup of the BR and estimate the time required for the BR to reach steady state. This simulation was carried out for a number of reasons:

- It was used to support the assumption that the COD components present in the seed sludge, which were absent in the synthetic feed, would be washed out of the BR.

- It was used to show that at steady state, the BR WAS consisted of only active biomass and decay products.

- It was used to estimate the date when the BR reached steady state. This was required to select the startup date of the AD. The AD needed to be started up with a stable BR WAS in order to minimize the time required for the $\mathrm{AD}$ to reach steady state.

- A stable source of BR WAS was required for pretreatment in order to accurately characterize the impacts of pretreatment.

As mentioned in section 3.3.1, the BR was initially seeded with $5 \mathrm{~L}$ of activated sludge from the Waterloo WWTP. On the date the sample was taken from the WWTP, the TCOD of the activated sludge reported by the plant was $3280 \mathrm{mg} / \mathrm{L}$. The SRT of the aeration basin from which the sample was withdrawn was reported to be approximately $8 \mathrm{~d}$. Using these values, the aeration basin of the Waterloo WWTP was simulated in BioWin 3.1 to estimate the concentration of the major COD contributors in the activated sludge. It was assumed that the plant received a continuous flow of typical settled medium-strength municipal wastewater that contained $450 \mathrm{mg} / \mathrm{L}$ TCOD, $15 \mathrm{mg} / \mathrm{L} \mathrm{ISS}$ and $60 \mathrm{mg} / \mathrm{L}$ non-biodegradable particulate COD $\left(\mathrm{X}_{\mathrm{i}}\right)$. The MLSS of the AS therefore included a mixture of ISS and VSS. The simulation was run until steady state conditions were reached. The major COD contributors in the AS were predicted to be particulate inert $\operatorname{COD}\left(\mathrm{X}_{\mathrm{i}}\right)$, endogenous products $\left(\mathrm{Z}_{\mathrm{e}}\right)$, and ordinary heterotrophic biomass that does not accumulate phosphorus $\left(\mathrm{Z}_{\mathrm{bh}}\right)$.

In a separate simulation, the bench scale BR was then modeled as an SBR with a $5 \mathrm{~d}$ SRT. The activated sludge from the Waterloo WWTP predicted in the previous simulation was used as the seed sludge in the BR. The influent parameters were specified according to the theoretical composition of 
the synthetic feed used from day 0 to day 179 of the project, as shown in Table 4.1. The COD:N:P ratio of the synthetic wastewater was 100: 4.4: 1.5. This is very close to the conventionally recommended ratio of 100: 5: 1 for aerated activated sludge systems.

Table 4.1 Influent Synthetic Wastewater Parameters

\begin{tabular}{|l|l|l|l|l|}
\hline \multirow{2}{*}{ Parameter } & \multicolumn{2}{|l|}{ Based on Synthetic Wastewater Recipe } & \multicolumn{2}{l|}{ Converted to Required Units for BioWin 3.1® } \\
\cline { 2 - 5 } & Concentration & Units & Concentration & Units \\
\hline $\mathrm{COD}$ & 8000 & $\mathrm{mg} / \mathrm{L} \mathrm{COD}$ & 8000 & $\mathrm{mg} / \mathrm{L} \mathrm{COD}$ \\
\hline $\mathrm{TKN}$ & 1330 & $\mathrm{mg} / \mathrm{L} \mathrm{NH} \mathrm{NH}_{4} \mathrm{Cl}$ & 350 & $\mathrm{mgN} / \mathrm{L}$ \\
\hline $\mathrm{TP}$ & 530 & $\mathrm{mg} / \mathrm{L} \mathrm{KH}_{2} \mathrm{PO}_{4}$ & 120 & $\mathrm{mgP} / \mathrm{L}$ \\
\hline $\mathrm{SCa}$ & 900 & $\mathrm{mg} / \mathrm{L} \mathrm{CaCl}_{2} * 2 \mathrm{H}_{2} \mathrm{O}$ & 245 & $\mathrm{mgCa}{ }^{2+} / \mathrm{L}$ \\
\hline $\mathrm{SMg}$ & 600 & $\mathrm{mg} / \mathrm{L} \mathrm{MgCl}_{2} * 6 \mathrm{H}_{2} \mathrm{O}$ & 72 & $\mathrm{mgMg}^{2+} / \mathrm{L}$ \\
\hline Alkalinity & 333 & $\mathrm{mg} / \mathrm{L} \mathrm{NaHCO}_{3}$ & 4 & $\mathrm{mmol} / \mathrm{L}$ \\
\hline
\end{tabular}

Figure 4.1 shows the simulated COD fractionation in the activated sludge in the BR during the first 3 days of operation. In a given day, the biodegradable COD fractions sharply increased when the reactor was fed, corresponding to a sharp increase in TCOD. The active organisms $\left(\mathrm{Z}_{\mathrm{bh}}\right)$ underwent rapid growth, and then began to decline when the substrate was depleted. The endogenous products $\left(Z_{e}\right)$ steadily increased during the reaction period and began to accumulate in the reactor. When the reactor was allowed to settle, the particulate COD concentrations dropped to zero because the model assumed perfect settling.

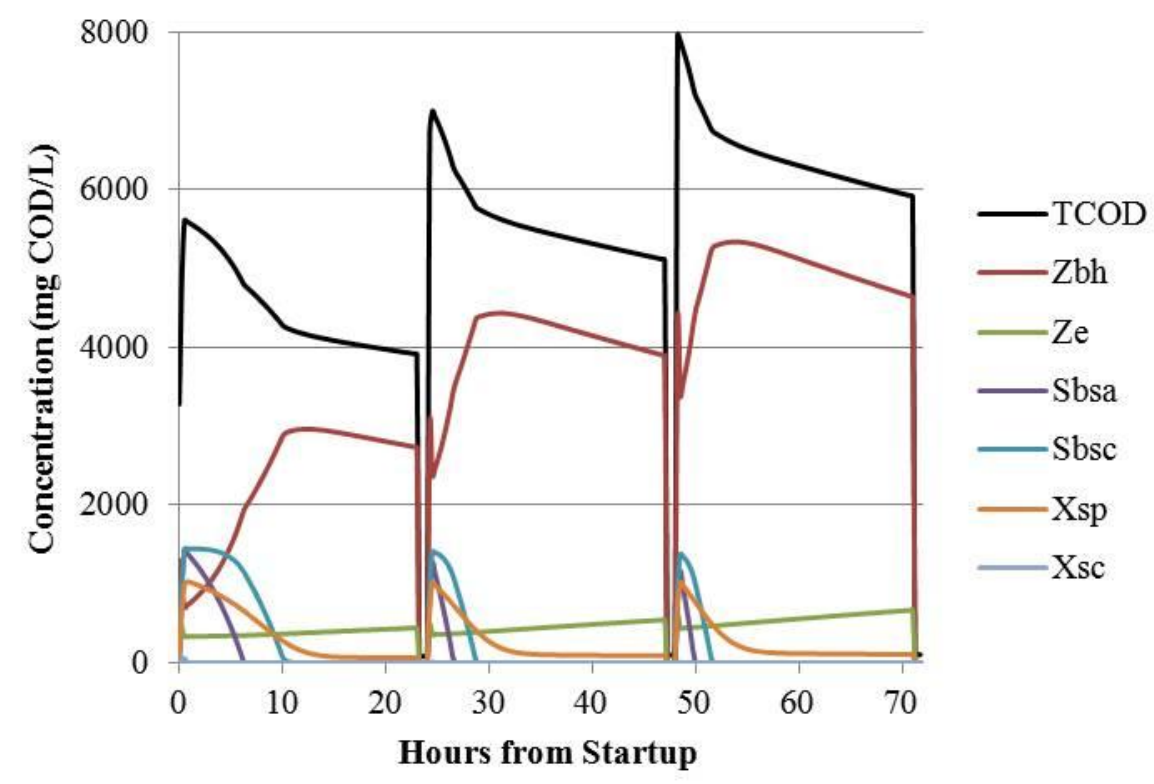

Figure 4.1 Simulated COD Fractionation of WAS in BR during Startup 
The active heterotroph concentration in the BR at the end of the reaction period was predicted to drastically increase during the first three days of operation, as shown in Figure 4.1. This was due to the relatively high strength of the synthetic feed compared to the assumed strength of the municipal wastewater at the Waterloo WWTP. Consequently, the predicted $Z_{e}$ concentration also steadily increased in the BR. The model predicted that $X_{i}$ would be washed out of the BR within 3 SRTs or 15 days. As shown in Figure 4.2, the TCOD of the WAS was predicted to be almost entirely comprised of $Z_{b h}$ and $Z_{e}$ at steady state conditions which also occurred within 3 SRTs. The TCOD of the BR WAS was measured on day 15 and was found to be $7700 \pm 150 \mathrm{mg} / \mathrm{L}$. In this report, a number preceded by the symbol \pm indicates one standard deviation above and below the mean of a sample set. The predicted TCOD of the BR WAS at the end of the reaction period on this day was $7790 \mathrm{mg} / \mathrm{L}$ which matched the measured value.

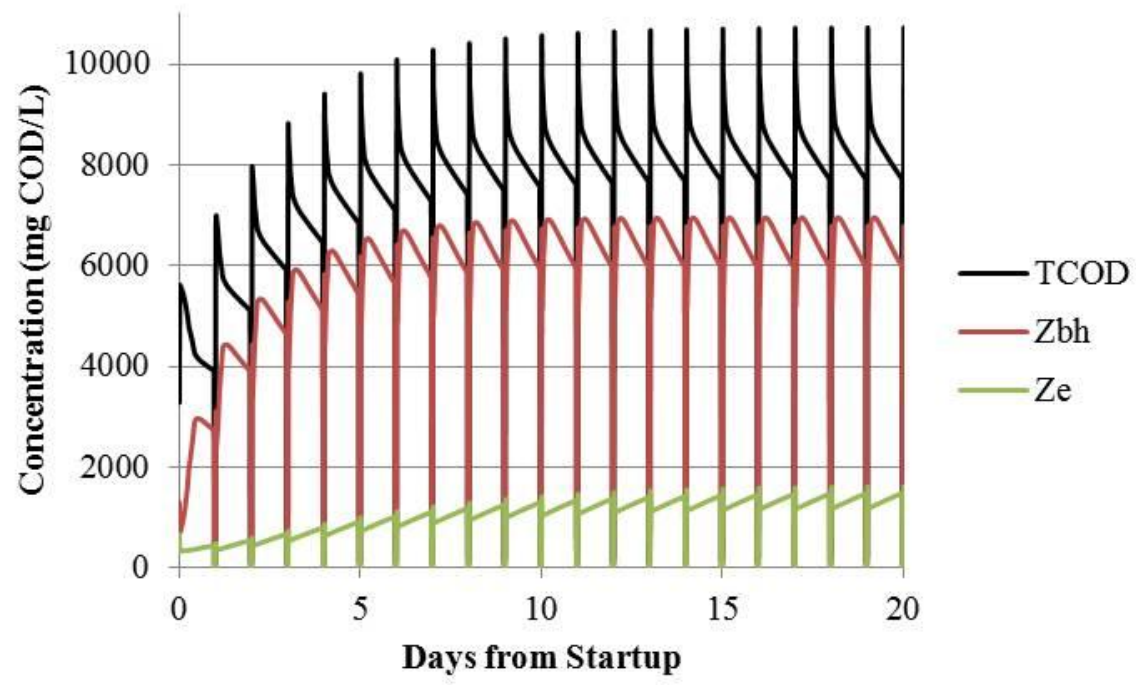

Figure 4.2 COD Fractionation of WAS in BR Over 20 Days

As mentioned in section 3.2, the synthetic feed concentration was halved on day 179. It was predicted using another BioWin simulation that a new steady state condition would be reached within 3 SRTs following this feeding change. After day 194, the predicted steady-state TCOD, $\mathrm{Z}_{\mathrm{bh}}$ and $\mathrm{Z}_{\mathrm{e}}$ concentrations in the BR were half of the corresponding steady-state values shown in Figure 4.2.

In conclusion, the simulation work presented in this section corroborated the findings of Ramdani et al (2010) that showed the activated sludge fed with synthetic substrate was comprised of only active biomass and decay products. The BR was predicted to reach steady state within 3 SRTs from startup. It was therefore believed that when the AD was started up with BR WAS on day 20, it was fed with a stable WAS source. Based on the simulations, the BR WAS was predicted to be changing in 
composition from day 0 to day 15 and from day 179 until day 194. Pretreatment experiments were therefore not carried out during these periods.

\subsection{Physical and Biochemical Characterization of Process Streams}

\subsubsection{Synthetic Wastewater}

The concentration of conventional parameters in the synthetic feed obtained from the physical and biochemical analyses are presented in Table 4.2. These values represent the concentrations observed throughout Phases 1 and 2 of the project, i.e. day 179 until day 283.

Table 4.2 Concentration of Conventional Parameters in Synthetic Wastewater

\begin{tabular}{|c|c|c|}
\hline Parameter & Avg. Concentration (mg/L) & Std. Dev. (mg/L) \\
\hline TCOD & 4006 & 183 \\
\hline SCOD & 2983 & 73 \\
\hline ffCOD & 2751 & 117 \\
\hline ISS & 100 & 61 \\
\hline VSS & 833 & 57 \\
\hline pH & 4.5 & 0.1 \\
\hline
\end{tabular}

The COD of the synthetic feed was fractionated into the components employed by the BioWin 3.1 Integrated Model, as shown in Figure 4.3. This allowed the synthetic substrate to be accurately represented in the Phase 1 and 2 system simulations which will be presented in section 5. As shown in Table 4.2, suspended solids measurements of the synthetic feed revealed the presence of $100 \pm 61$ $\mathrm{mg} / \mathrm{L}$ of ISS. Hence the AS in the BR contained some ISS at steady-state operation and thus deviated slightly from the ideal composition presented in Figure 2.1. This ISS probably originated from the yeast extract. The average measured ffCOD concentration in the BR WAS was $53 \pm 12 \mathrm{mg}$ COD/L and this was assumed to be soluble microbial products (SMPs) generated by the microorganisms in the reactor. As explained by Kianmehr (2010), SMPs are soluble non-biodegradable COD. Therefore the net COD that bacteria converted in the BR was the measured TCOD of the synthetic feed less the measured ffCOD of the BR WAS which was $98.7 \pm 6.1 \%$ of the TCOD of the synthetic substrate. The activated sludge models used in this project are not capable of predicting the 
channeling of COD into SMPs. Therefore, for the purpose of modeling, the remaining $1.3 \%$ was assigned as $S_{\text {us }}$ in the synthetic feed, as shown in Figure 4.3. The slowly biodegradable COD fraction $(25.5 \%)$ was obtained by dividing the average measured particulate COD by the TCOD. It was assumed that $75 \%$ of the slowly biodegradable COD was particulate $\left(\mathrm{X}_{\mathrm{sp}}\right)$ and the remainder was colloidal $\left(\mathrm{X}_{\mathrm{sc}}\right)$. The readily biodegradable COD fraction was obtained by subtracting the nbCOD and sbCOD from the TCOD. As was shown in Table 3.1, half of the synthetic feed TCOD was from acetate hence the $S_{\text {bsa }}$ fraction was assigned to be half the rbCOD. The remaining rbCOD was assigned as complex $\left(\mathrm{S}_{\mathrm{bsc}}\right)$.

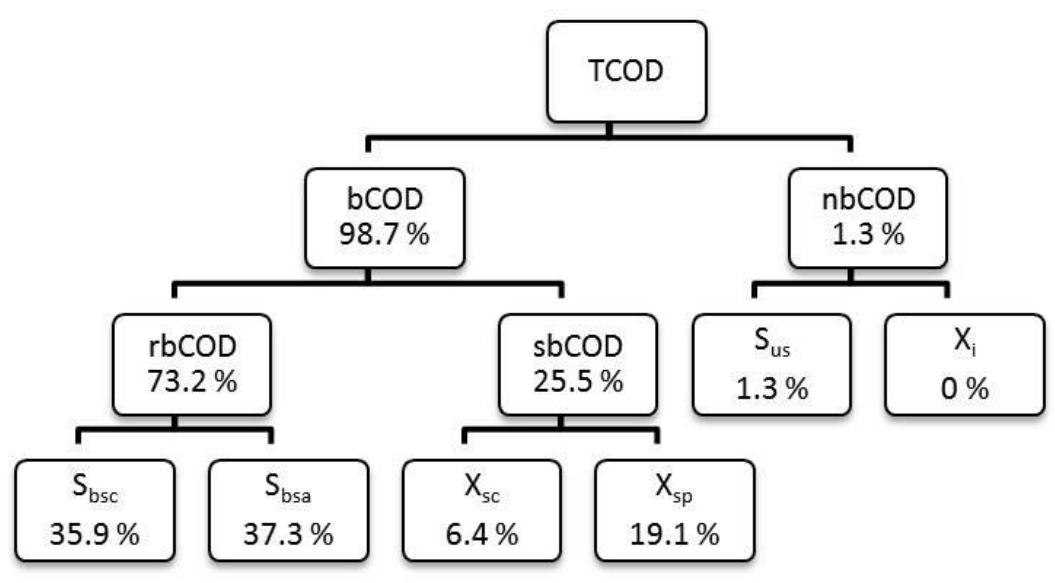

Figure 4.3 COD Fractionation of Synthetic Wastewater

\subsubsection{Biological Reactor}

As mentioned in section 4.1, the synthetic feed concentration was halved on day 179 and the BR reached steady state within 3 SRTs following this feeding change. As shown in Figure 4.4, the concentrations of the COD and SS components measured by conventional analyses remained relatively stable from day 197 until the end of the project, day 283. It was therefore concluded that the BR was at steady state during this period 


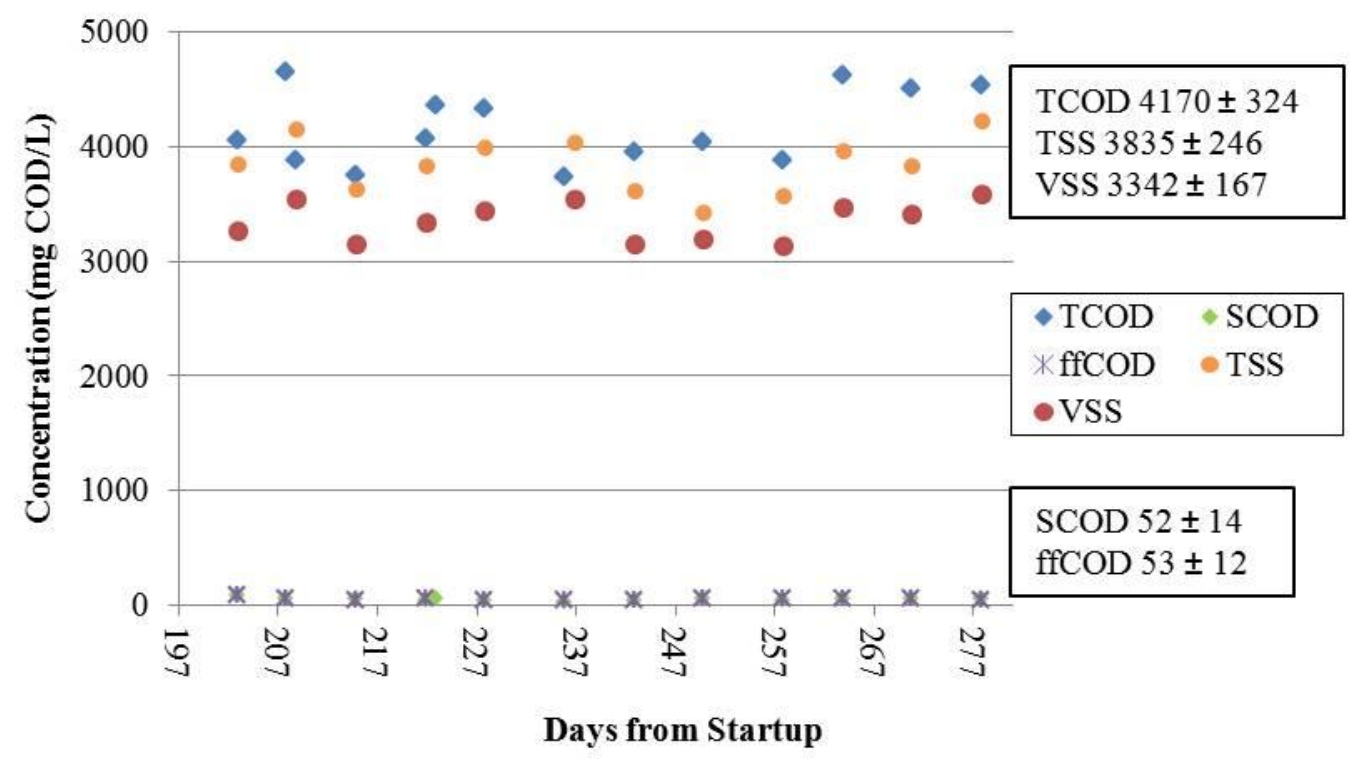

Figure 4.4 COD and SS Concentrations in BR WAS

The COD of the BR WAS was expected to be entirely comprised of biomass and associated products because the synthetic substrate was believed to be entirely oxidized within the reaction period in the BR. This was shown in the reactor simulation presented in section 4.1. Furthermore, the DO concentration in the BR dropped to almost zero immediately after the reactor was fed and then increased above 2 ppm within two hours, indicating that most of the substrate was depleted within this time.

The data presented in Figure 4.4 was used to estimate several properties of the BR WAS. The BR WAS contained an average ffCOD concentration of $53 \pm 12 \mathrm{mg} / \mathrm{L}$ and this was assumed to be $S_{\text {us }}$ from SMPs generated in the reactor. In addition, colloidal COD was not present in the BR WAS because there was no significant difference between the measured SCOD and ffCOD concentrations. As explained by Kianmehr (2010), colloidal COD in WAS is typically comprised of extra cellular polymeric substances (EPS). Therefore the BR WAS did not contain EPS that was in the colloidal range. Besides the relatively small concentration of SMPs, the COD of the BR WAS was therefore entirely particulate.

The average measured COD/VSS ratio of the BR WAS was calculated to be $1.23 \pm 0.08$. This was less than the typical value of 1.42 (Henze et al., 2008) for active heterotrophs and endogenous residue. The biomass in the $\mathrm{BR}$ was therefore more oxidized than typical biomass and it was hypothesized that this suggested the presence of stored COD such as glycogen or poly-hydroxy- 
alkanoates (PHA). Although stored COD is not directly identifiable chemically, it may be recovered in COD analysis and must satisfy COD conservation. The COD/VSS ratio of glycogen, a polysaccharide, has been reported to be 1.1 gCOD/gVSS (Ramdani et al., 2012). Therefore the presence of glycogen in addition to active heterotrophs and decay products in the BR WAS would lower the average COD/VSS ratio below 1.42 .

The following section presents the derivation of a mass balance that was conducted to estimate the fraction of the biomass COD which was contributed by storage products. In equations 4.1, 4.2 and 4.3 below, the COD/VSS ratio of biomass is described by $\Upsilon_{\mathrm{Z}}$, the COD/VSS ratio of stored COD is defined as $\Upsilon_{\text {STO }}$ and the average measured COD/VSS ratio of the BR WAS is defined as $\Upsilon_{\text {OBS. }}$ Assuming that stored COD $\left(\mathrm{X}_{\text {STO }}\right)$ was present in the BR WAS and had an associated $\Upsilon_{\text {STO }}$ ratio and that the only other COD components in the BR WAS were active heterotrophs $\left(\mathrm{Z}_{\mathrm{bh}}\right)$ and endogenous residue $\left(Z_{\mathrm{e}}\right), \Upsilon_{\mathrm{OBS}}$ may be expressed by equation 4.1 .

$$
\Upsilon_{O B S}=\frac{X_{S T O}+Z_{b h}+Z_{e}}{\frac{X_{S T O}}{\Upsilon_{S T O}}+\frac{Z_{b h}+Z_{e}}{\Upsilon_{Z}}}
$$

The fraction of the TCOD that was present as storage products $\left(\mathrm{f}_{\mathrm{STO}}\right)$ was equal to the ratio $\frac{X_{S T O}}{T C O D}$ and the TCOD was the sum of $\mathrm{X}_{\mathrm{STO}}, \mathrm{Z}_{\mathrm{bh}}$ and $\mathrm{Z}_{\mathrm{e}}$. These terms were substituted into equation 4.1 and it was rearranged to yield equation 4.2.

$$
\Upsilon_{O B S}=\frac{\Upsilon_{Z} \Upsilon_{S T O}}{\Upsilon_{Z} f_{S T O}+\Upsilon_{S T O}\left(1-f_{S T O}\right)}
$$

Rearranging equation 4.2 to solve for $\mathrm{f}_{\mathrm{STO}}$ yielded equation 4.3 .

$$
\mathrm{f}_{\mathrm{STO}}=\frac{\Upsilon_{\mathrm{STO}} \Upsilon_{\mathrm{Z}}-\mathrm{Y}_{\mathrm{OBS}} \Upsilon_{S T O}}{\Upsilon_{O B S} \Upsilon_{Z}-\Upsilon_{O B S} \Upsilon_{S T O}}
$$

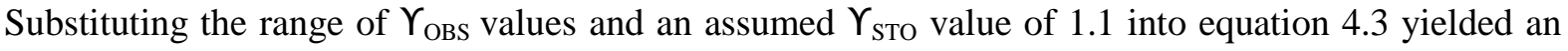
average $\mathrm{f}_{\text {Sто }}$ value of $54 \pm 28 \%$. The results of this calculation indicated that more than half of the TCOD of the BR WAS was contributed by storage products. Therefore, the heterotrophs in the BR did not use all of the synthetic biodegradable substrate for growth within the $5 \mathrm{~d}$ SRT and the concentration of active biomass in the BR was less than expected. In a similar experiment, Ramdani et al. (2012) used an MBR with a $5.2 \mathrm{~d}$ SRT to treat a completely biodegradable synthetic influent comprised of sodium acetate as the sole carbon source. These authors reported that the $\mathrm{Z}_{\mathrm{bh}}$ and $\mathrm{Z}_{\mathrm{e}}$ 
fractions were 68 and $32 \%$ respectively. Assuming an $\mathrm{f}_{\mathrm{STO}}$ value of $54 \%$, the sum of $\mathrm{Z}_{\mathrm{bh}}$ and $\mathrm{Z}_{\mathrm{e}}$ in the BR WAS was only $46 \%$ of the biomass COD. The composition of the BR WAS therefore deviated from the composition reported by Ramdani et al. (2010) in Figure 2.1. It is important to note that the

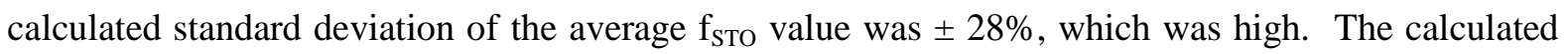
$f_{\text {STO }}$ ratio was found to be very sensitive to the $\Upsilon_{\text {OBS }}$ value.

Because the AD was fed with BR WAS, some properties of the AD WAS could be estimated based on the properties of the BR WAS. First, the COD of the BR WAS consisted of $Z_{b h}, Z_{e}$ and $X_{\text {STO }}$ hence the AD WAS was expected to be comprised of biomass and associated products. Furthermore, it was expected that the $\mathrm{X}_{\mathrm{STO}}$ present in the BR WAS would be assimilated to AD biomass because the digester had an SRT twice as long as that in the BR. Thus the AD WAS was expected to be mainly comprised of $Z_{b h}$ and $Z_{e}$. In addition the AD WAS was expected to contain concentrations of SCOD and ffCOD similar to those measured in the BR WAS. It was hypothesized that the small concentration of SMPs believed to be present in the BR WAS were generated by biomass growth. Because biomass growth was not expected to occur in the AD, additional SMPs were therefore not expected to be generated in the AD. The properties of the WAS from the AD will be discussed further in section 4.2.4.

\subsubsection{Effects of Pretreatment on BR WAS}

The $\mathrm{pH}$ was measured in the BR throughout the project as one way of assessing the stability of the reactor. The $\mathrm{pH}$ of the BR WAS remained relatively constant throughout Phases 1 and 2 of the project, as shown in Figure 4.5, indicating reactor stability. Pretreatment slightly decreased the $\mathrm{pH}$ of the WAS from an average of $8.5 \pm 0.1$ to $7.8 \pm 0.2$. Both the original and lower $\mathrm{pH}$ values were within the range reported by Tchobanoglous et al. (2003) for uninhibited heterotroph activity hence $\mathrm{pH}$ adjustment was not required before the pretreated WAS was fed to the $\mathrm{AD}$. This $\mathrm{pH}$ decrease was expected as it has been shown that HPTH pretreatment produces volatile fatty acids (MorganSagasume et al., 2010). 


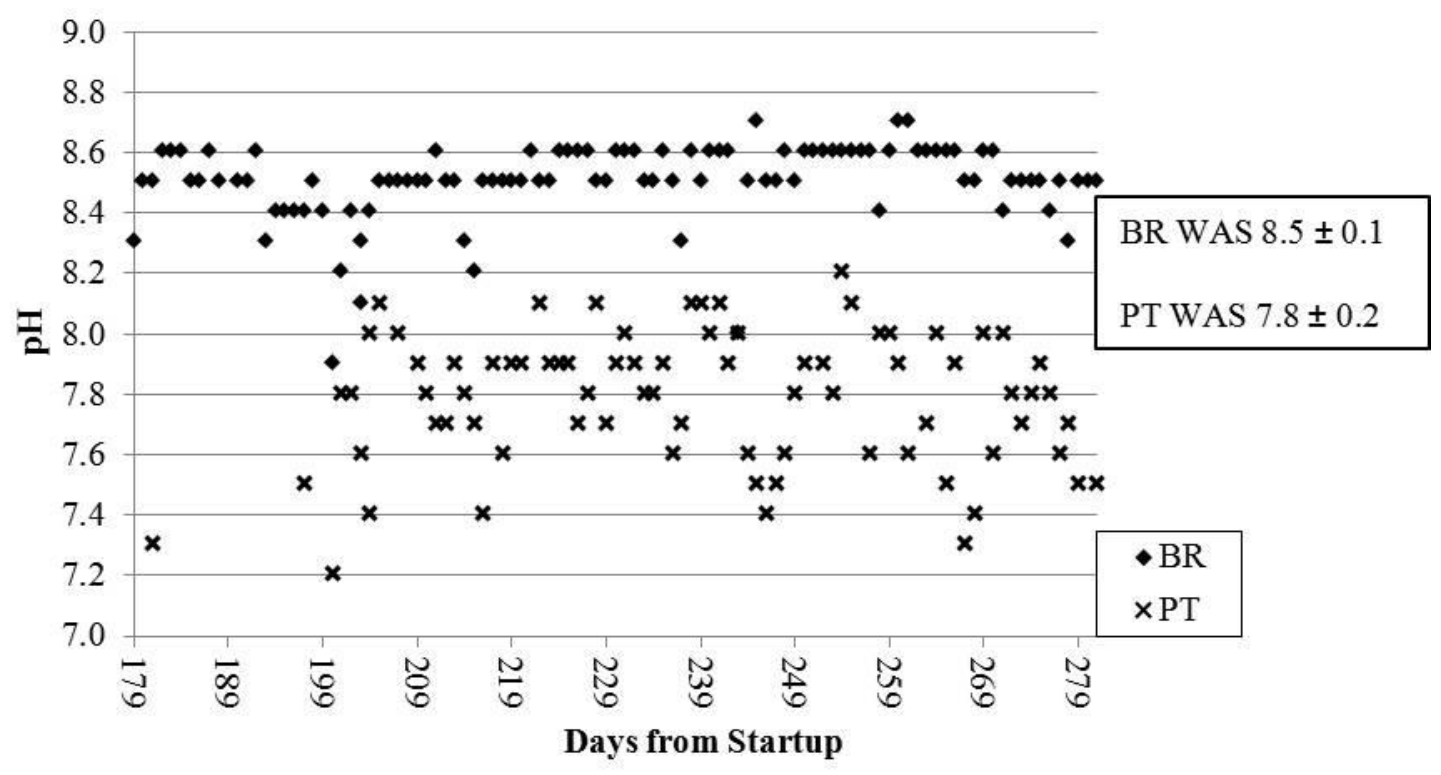

Figure 4.5 Measured pH in Raw and Pretreated BR WAS

Pretreatment substantially solubilized COD, as was expected based on the results of published HPTH pretreatment studies. The average concentration of each COD species in the raw and pretreated WAS was calculated over the period from day 194 to 283 , i.e. while the BR was operating at steady state. For each measurement, the PCOD was calculated by subtracting the SCOD from the TCOD. As shown in Figure 4.6, the sCOD and ffCOD concentrations calculated using equation 2.2 increased by $56 \pm 7 \%$ and $41 \pm 5 \%$ respectively due to pretreatment. By comparison, the range of COD solubilization reported in the HPTH pretreatment studies referenced in section 2.1 .4 was 28 to $45 \%$. In these studies, the pretreatment temperature ranged from 150 to $170^{\circ} \mathrm{C}$ and the soluble COD was defined as that passing through filters with pore diameters ranging from 0.45 to $1.6 \mu \mathrm{m}$. The pressure, duration of heating and mode of operation also differed among the referenced studies. Therefore a direct comparison between the COD solubilization values measured in this project and those measured in the referenced research could not be made. The BR WAS did not contain colloidal COD whereas the difference between the SCOD and fFCOD measurements in the pretreated WAS showed that it contained a colloidal COD concentration of $644 \pm 285 \mathrm{mg} / \mathrm{L}$. Kianmehr (2010) suggested that the increase in colloidal COD due to pretreatment could result from the solubilization of extra cellular polymeric substances (EPS). 


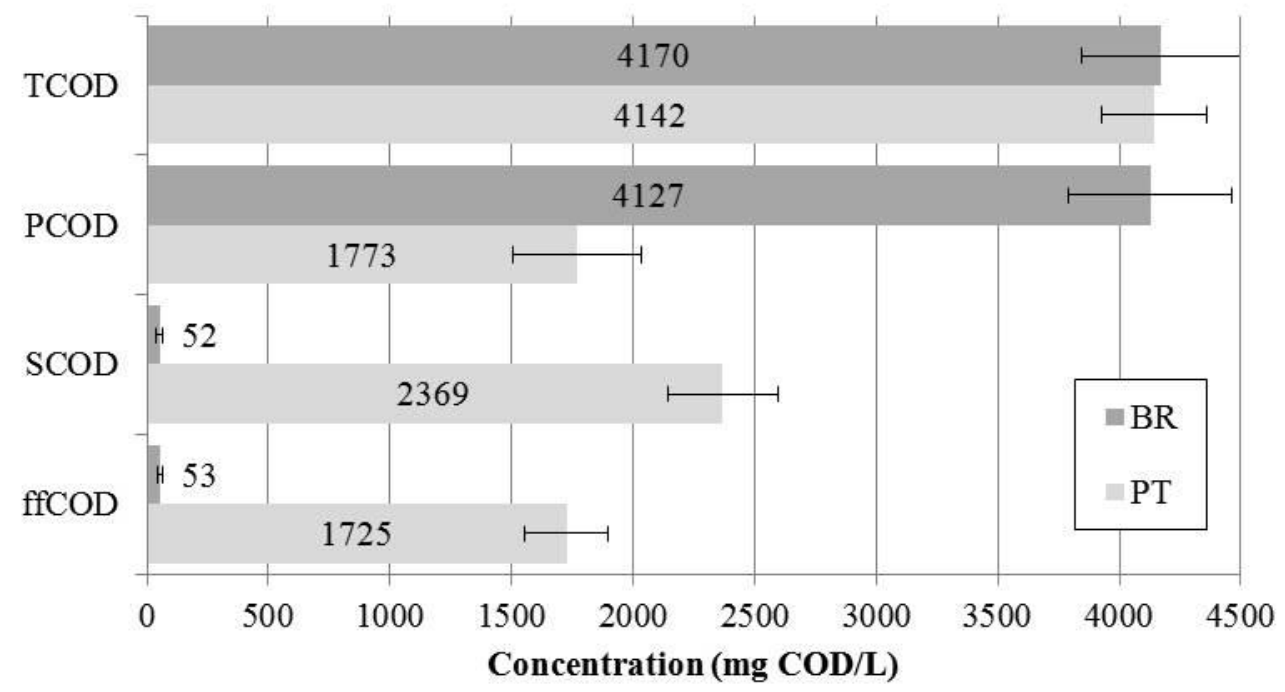

Figure 4.6 Average COD Concentrations in Raw and Pretreated BR WAS

As shown in Figure 4.6, the TCOD concentration remained unchanged by pretreatment, indicating no significant removal of organic matter occurred. This was consistent with the results of MorganSagasume et al. (2010) where TCOD was observed to be conserved during CAMBI ${ }^{\mathrm{TM}}$ pretreatment. Pretreatment caused a noticeable colour change in the WAS in both the supernatant and the settled sludge. The colour changed from light brown to dark brownish purple. Because the COD was measured by spectrophotometry, it was necessary to determine whether this colour change affected the COD measurement. Samples of raw and pretreated WAS were prepared at the same dilution factor. Four replicate bottles of each sample were prepared and a potassium hydrogen phthalate solution of a known COD concentration was added to two of the bottles. Four replicate bottles containing only the COD standard were also prepared. The bottles were then analyzed for COD according to the method described in 3.5.2. For each bottle containing dosed COD and WAS, the COD of the standard was calculated as the measured TCOD of this bottle less the measured COD of the bottle containing WAS only. The results showed no significant difference in the measured COD of the standard among all the bottles, including the bottles containing only standard. This test was repeated for filtered WAS samples and again no significant difference in the measured COD of the standard was observed among all the bottles. Therefore the colour change imparted by pretreatment to the supernatant and sludge did not interfere with the COD measurement.

Suspended solids measurements were carried out on the raw and pretreated BR WAS on 12 separate days during Phases 1 and 2. On the basis of these measurements it was concluded that the organic suspended solids were preferentially solubilized by pretreatment as compared to the inorganic 
suspended solids. As shown in Figure 4.7, the average VSS concentrations before and after pretreatment were $3342 \pm 167 \mathrm{mg} / \mathrm{L}$ and $1518 \pm 230 \mathrm{mg} / \mathrm{L}$, respectively. A t-test at the $95 \%$ confidence level revealed that pretreatment caused the VSS concentration to decrease by $1824 \pm 284$ $\mathrm{mg}$ VSS/L. The average ISS concentrations before and after pretreatment were $489 \pm 103 \mathrm{mg} / \mathrm{L}$ and $412 \pm 131 \mathrm{mg} / \mathrm{L}$, respectively, and a t-test at the $95 \%$ confidence level showed no significant difference between these average values.

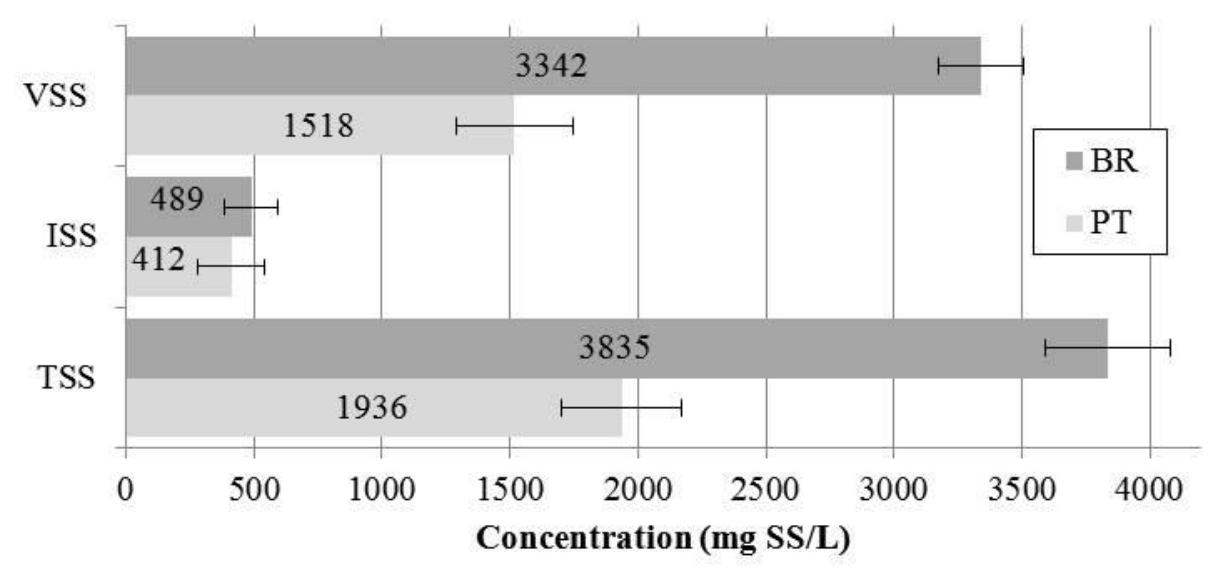

Figure 4.7 Average SS Concentrations in Raw and Pretreated BR WAS

In a study of the $\mathrm{CAMBI}^{\mathrm{TM}}$ pretreatment technology, Morgan-Sagasume et al. (2010) reported that pretreatment caused the TSS concentration to decrease between 20 and 30\%, according to equation 4.4:

$$
\text { TSS decrease } \%=\frac{T S S_{B R}-T S S_{P T}}{T S S_{B R}} \times 100 \%
$$

To compare the results of this study to those reported by these authors, the decrease in the TSS concentration due to pretreatment was calculated for each of the 12 sampling days. The average decrease in TSS concentration due to pretreatment was $49 \pm 6 \%$ which was higher than that reported by Morgan-Sagasume et al. (2010). A t-test at the $95 \%$ confidence level showed that the average TSS concentration decreased $1899 \pm 205 \mathrm{mg} / \mathrm{L}$ due to pretreatment in the current study.

Bougrier et al. (2008) reported a 9\% decrease in the VSS/TSS ratio of a sludge that was pretreated at $150^{\circ} \mathrm{C}$. In order to compare the results of this study to those reported by these authors, the VSS/TSS ratio was calculated for the raw and pretreated WAS on each day the suspended solids were measured. The average VSS/TSS ratio before and after pretreatment was $87 \pm 15 \%$ and $77 \pm 8 \%$, respectively. A t-test at the $95 \%$ confidence level showed no significant difference between these two averages. However, at the $90 \%$ confidence level, the VSS/TSS ratio decreased $10 \pm 9 \%$ due to pretreatment which is comparable to the findings of Bougrier et al. (2008). 
On each sampling day, the VSS destruction was calculated according to equation 4.5 using the measured VSS concentration of the BR WAS $\left(\mathrm{VSS}_{\mathrm{BR}}\right)$ and pretreated BR WAS (VSS $\left.\mathrm{PT}_{\mathrm{P}}\right)$. The average VSS destruction due to pretreatment was found to be $56 \pm 10 \%$. In summary, the bench-scale pretreatment used in this project was deemed to be representative of full-scale processes because the measured effects of pretreatment on $\mathrm{pH}, \mathrm{SS}$ and $\mathrm{COD}$ species were found to be comparable to similar published research.

VSS destruction $\%=\frac{V S S_{B R}-V S S_{P T}}{V S S_{B R}} \times 100 \%$

The concentrations of various nitrogen species before and after pretreatment are presented in Figure 4.8. The concentrations of these species were measured in the raw and pretreated WAS on 7 separate days during Phases 1 and 2. The concentrations of ammonia and nitrate in both types of WAS were measured on 5 additional days.

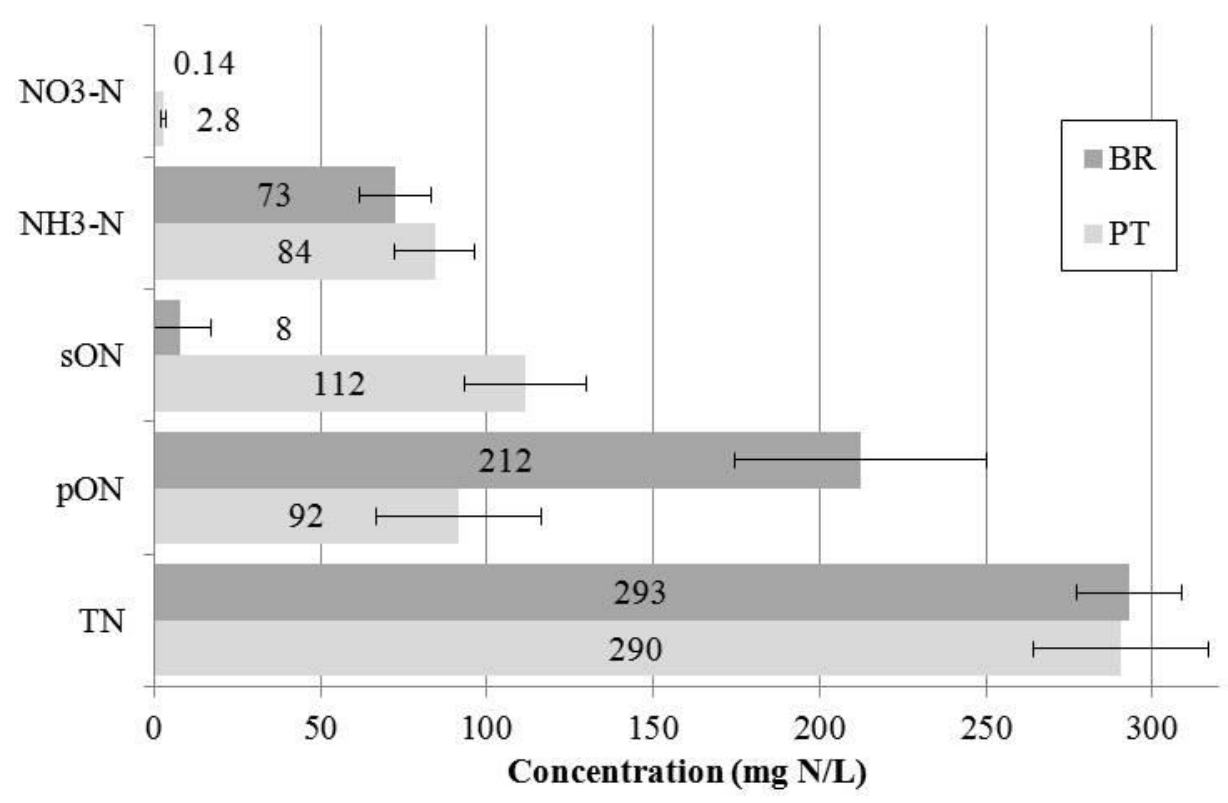

Figure 4.8 Average Concentration of Nitrogen Species in Raw and Pretreated BR WAS

A t-test at the 95\% confidence level showed that the total nitrogen (TN) which included nitrate, ammonia and soluble and particulate organic nitrogen was not significantly altered by pretreatment. Another t-test at the 95\% confidence level showed that the ammonia concentration was not significantly changed by pretreatment. This indicates that significant mineralization of organic nitrogen did not occur. By comparison, Bougrier et al. (2008) and Donoso-Bravo et al. (2010 b) both reported that HPTH pretreatment slightly increased the ammonia concentration. Because both the total nitrogen and ammonia concentrations were unaltered by pretreatment, the organic nitrogen (ON) 
concentration also remained unchanged. However, as shown in Figure 4.8, ON was solubilized. The average ON solubilization was $49 \% \pm 11 \%$, as calculated according to equation 4.6.

$$
\text { ON solubilization } \%=\frac{\operatorname{sON}_{P T}-s O N_{B R}}{p O N_{B R}} \times 100 \%
$$

The nitrogen content of the organics was calculated by dividing the measured particulate $\mathrm{ON}$ (pON) by the measured particulate COD (PCOD). The average pON/PCOD ratios before and after pretreatment were found to equal $0.053 \pm 0.006 \mathrm{mgN} / \mathrm{mgCOD}$ and $0.052 \pm 0.016 \mathrm{mgN} / \mathrm{mgCOD}$, respectively. A t-test revealed that there was no significant different difference between these means at the $95 \%$ confidence level. This indicates that all types of particulate organics were solubilized to the same extent by pretreatment.

The measured pON/PCOD values were less than the typical value of $0.07 \mathrm{mgN} / \mathrm{mgCOD}$ reported by Henze et al. (2008) for active biomass and decay products which suggested the presence of storage products such as glycogen or PHA that do not contain nitrogen. A mass balance approach on the nitrogen species was therefore employed to estimate the contribution of storage products to the biomass COD. In equations 4.7 and 4.8 below, the measured pON/PCOD ratio was defined as $\mathrm{fN}_{\mathrm{OBS}}$, the typical pON/PCOD ratio for biomass was defined as $\mathrm{fN}_{\mathrm{Z}}$ and the pON/PCOD ratio for stored COD was defined as $\mathrm{fN}_{\text {Stо }}$. Assuming that $\mathrm{X}_{\text {STо }}$ was present and that the PCOD consisted of $\mathrm{X}_{\mathrm{STO}}$, $\mathrm{Z}_{\mathrm{bh}}$ and $\mathrm{Z}_{\mathrm{e}}$, the $\mathrm{fN}_{\mathrm{OBS}}$ value may be expressed by equation 4.7 .

$$
f N_{O B S}=\frac{f N_{S T O}+f N_{Z}\left(Z_{b h}+Z_{e}\right)}{X_{S T O}+Z_{b h}+Z_{e}}
$$

The value of $\mathrm{fN}_{\mathrm{STO}}$ was assumed to be zero and the fraction of the PCOD that was present as storage products $\left(\mathrm{f}_{\mathrm{STO}}\right.$ ) was equal to the ratio $\frac{X_{S T O}}{p C O D}$. Substituting these values into equation 4.7 and solving for $\mathrm{f}_{\text {STO }}$ yielded equation 4.8 .

$$
f_{S T O}=1-\left(\frac{f N_{O B S}}{f N_{Z}}\right)
$$

Substituting the range of $\mathrm{fN}_{\mathrm{OBS}}$ values measured in the $\mathrm{BR}$ into equation 4.8 yielded an average $\mathrm{f}_{\mathrm{STO}}$ value of $25 \pm 9 \%$. As mentioned in section 4.2.2, the average $\mathrm{f}_{\text {STO }}$ values calculated using equation 4.3 were $54 \pm 28 \%$. Thus the difference between the $\mathrm{f}_{\text {Sто }}$ values calculated using equations 4.3 and 4.8 was $29 \%$ and a t-test at the $95 \%$ confidence level revealed that this difference was significant. It is believed that the $\mathrm{f}_{\text {Sто }}$ values estimated using equation 4.8 were more reliable that those estimated using equation 4.3 because only one value $\left(\mathrm{fN}_{\mathrm{Z}}\right)$ was assumed in this approach whereas two values 
$\left(\Upsilon_{\mathrm{Z}}\right.$ and $\left.\Upsilon_{\mathrm{STO}}\right)$ were assumed in equation 4.3. The $\mathrm{f}_{\mathrm{STO}}$ values were shown to be sensitive to the assumed values in both equations. From a practical standpoint, it seems unlikely that the mass of COD stored within the cells could be much higher than the mass of the cells.

The soluble nitrogen $(\mathrm{sN})$ was calculated as the sum of the nitrate, ammonia and soluble organic nitrogen. Based on the calculated decrease in VSS concentration caused by pretreatment $\left(\mathrm{VSS}_{\text {destroyed }}\right)$ and the pON/PCOD and COD/VSS ratios measured in the BR WAS, the expected increase in soluble nitrogen due to pretreatment was $119 \pm 24 \mathrm{mgN} / \mathrm{L}$, as determined by equation 4.9.

$$
\text { Increase in } S N\left(\frac{m g N}{L}\right)=\frac{C O D}{V S S}_{\text {measured }} \times \frac{p O N}{p C O D}_{\text {measured }} \times V S S_{\text {destroyed }}
$$

Based on seven independent measurements of the nitrogen species in the raw and pretreated WAS, the average soluble nitrogen increase due to pretreatment was $118 \pm 26 \mathrm{mgN} / \mathrm{L}$. A t-test at the $95 \%$ confidence level showed no significant difference between the measured values and the expected values calculated by equation 4.9 .

In summary, the HPTH pretreatment employed in this study substantially solubilized the COD of the WAS while conserving the TCOD. Furthermore, organics were preferentially solubilized over inorganics. These findings are supported by the results of similar published research. Lastly, the analyses of the nitrogen species in this study indicated that pretreatment solubilized rather than mineralized proteins and that all types of particulate organics were solubilized to the same extent by pretreatment.

\subsubsection{Aerobic Digester}

The $\mathrm{pH}$ in the $\mathrm{AD}$ was measured throughout the project as one way of assessing the stability of the digester. The $\mathrm{pH}$ remained relatively constant during Phases 1 and 2 despite the fact that it was receiving feed with a lower $\mathrm{pH}$ in Phase 2 than in Phase 1. The measured $\mathrm{pH}$ of the AD WAS from day 179 until the end of the project is shown in Figure 4.9. The $\mathrm{pH}$ remained within the range reported by Tchobanoglous et al. (2003) for uninhibited heterotroph activity. During Phase 1, the average $\mathrm{pH}$ of the $\mathrm{AD}$ WAS was $8.43 \pm 0.09$ compared to $8.33 \pm 0.17$ during Phase 2 . A t-test at the 95\% confidence level revealed no significant difference between the Phase 1 and 2 average values. During Phases 1 and 2, the average $\mathrm{pH}$ of the AD WAS was $8.30 \pm 0.20$. As mentioned in section 
2.1.4, HPTH has been linked to the degradation of macromolecules into acidic compounds such as volatile fatty acids. The insignificant $\mathrm{pH}$ decrease in the AD WAS from Phase 1 to 2 suggests that the acidic compounds believed to be generated by the pretreatment process were biodegraded in the AD.

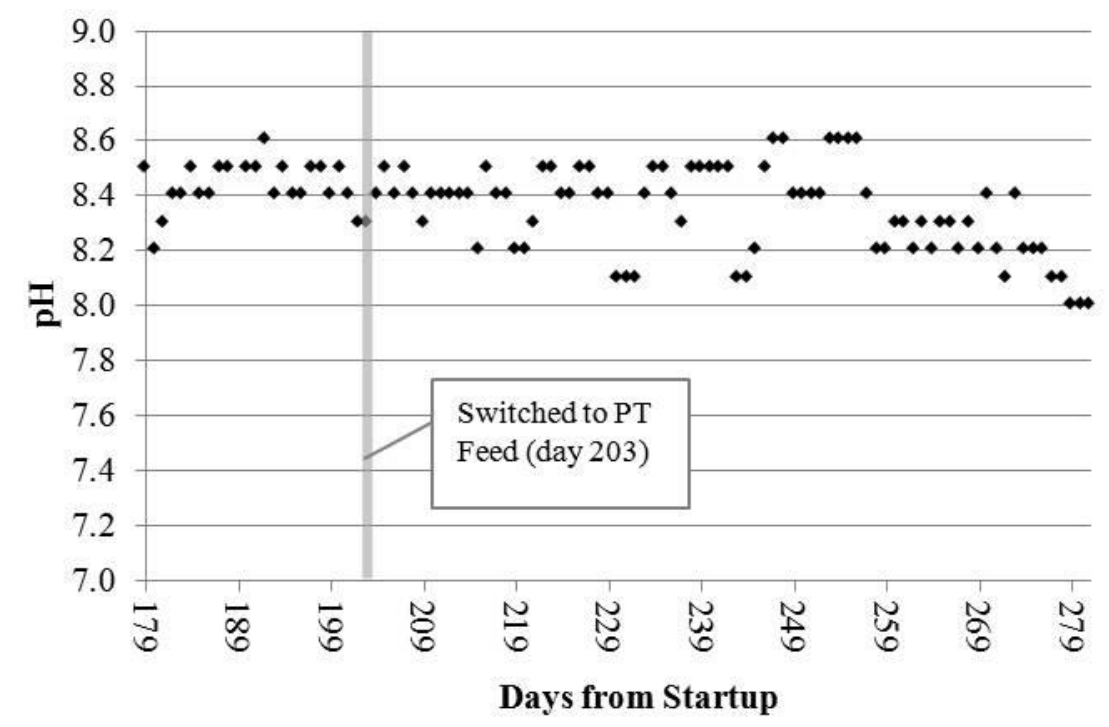

Figure 4.9 pH in AD WAS

The COD and SS species in the AD were measured by conventional analyses throughout Phases 1 and 2 of the project to assess the performance of the digester. As previously mentioned, the concentration of the synthetic substrate fed to the BR was halved on day 179. Consequently, the concentrations of the COD and SS species in both the BR and AD steadily decreased following this change. From day 197 until the end of the project the measured TCOD, SCOD, ffCOD and VSS concentrations in the $\mathrm{AD}$ remained relatively stable, despite the fact that the $\mathrm{AD}$ feed was switched from raw to pretreated BR WAS on day 203, i.e. at the beginning of Phase 2.

The average concentrations of the COD species were calculated for the stable period of Phase 1 (day 197 to 203) and Phase 2 (day 203 to day 283) and plotted in Figure 4.10. The average PCOD, SCOD and ffCOD concentrations decreased 12\%, 23\% and 7\%, respectively, from Phase 1 to 2 . T-tests at the 95\% confidence level showed that the decreases in PCOD and SCOD were significant whereas the decrease in ffCOD was insignificant. However, the decreases in PCOD and SCOD were considered relatively small. Because the measured ffCOD concentrations in the AD WAS were equivalent in Phases 1 and 2, it was deduced that pretreatment did not generate $S_{\text {us }}$ at the pretreatment temperature of $150^{\circ} \mathrm{C}$ that was employed in this study. This finding is in agreement with previous research which showed that HPTH pretreatment only produced refractory compounds that were 
essentially $\mathrm{S}_{\text {us }}$ when the temperature was above $150^{\circ} \mathrm{C}$ (Bougrier et al., 2007; Climent et al., 2007; Dwyer et al., 2008; Donoso-Bravo et al., 2010 b).

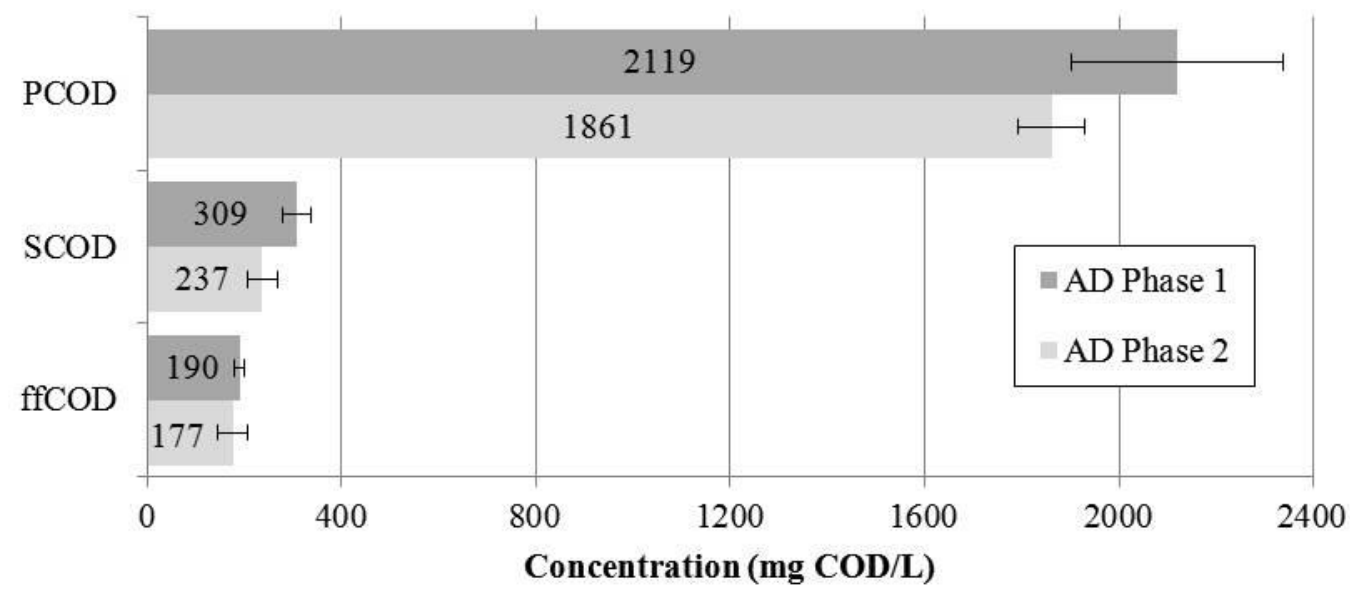

Figure 4.10 Average COD Concentrations in Phase 1 and 2 AD WAS

The observed stability of the measured TCOD, SCOD, ffCOD and VSS concentrations in the AD from day 197 until the end of the project is shown in Figure 4.11. During this period the average measured COD/VSS ratio of the AD WAS was calculated to be $1.44 \pm 0.11$. A t-test hypothesis at the $95 \%$ confidence level showed that this value was equivalent to the typical value of 1.42 for active biomass and decay products. This validated the assumption that the AD WAS consisted entirely of these two components during Phases 1 and 2. Hence, it was concluded that any storage products that may have been present in the BR WAS were depleted in the AD. 


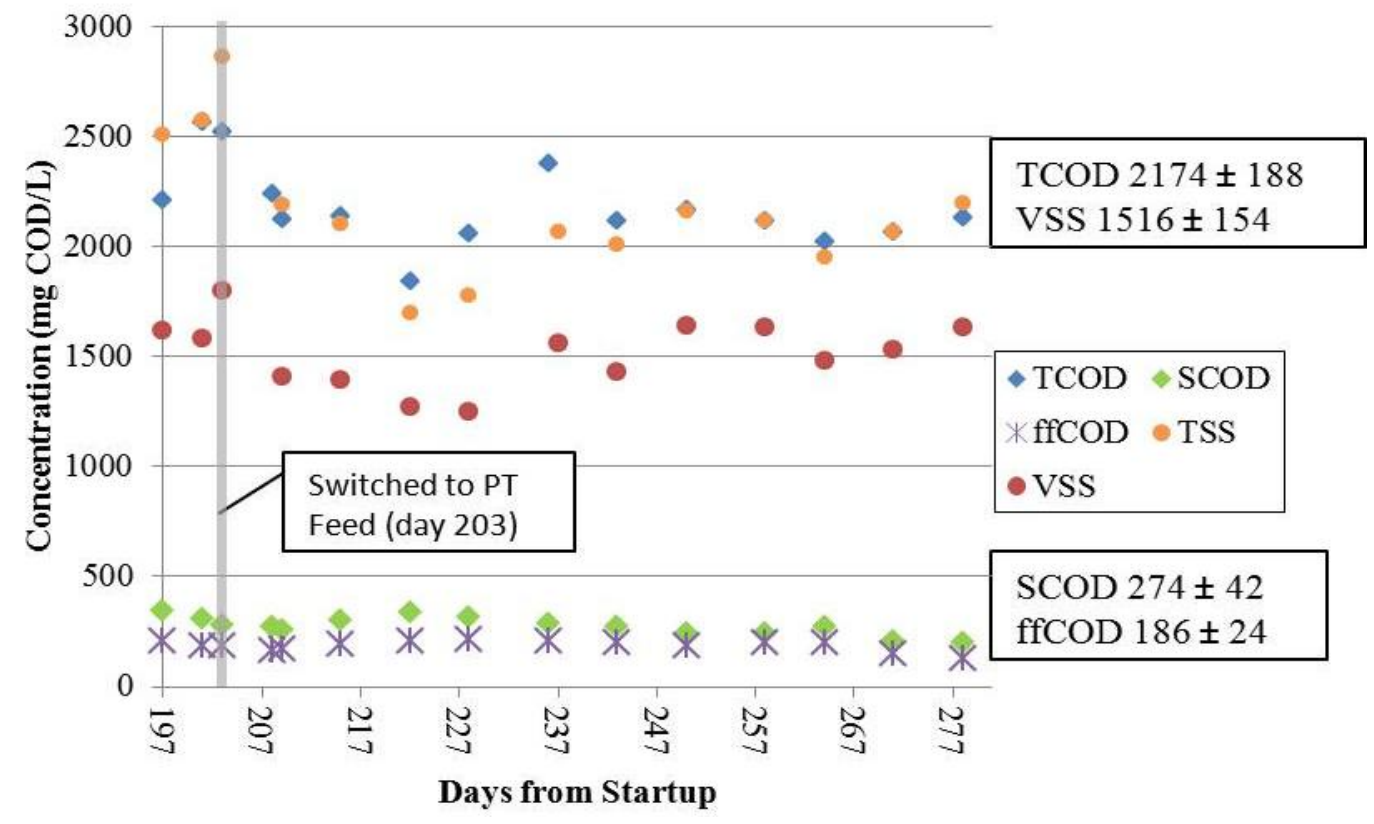

Figure 4.11 COD and SS Concentrations in AD WAS

The AD WAS contained some colloidal COD based on the data presented in Figure 4.11. A t-test at the $95 \%$ confidence level showed a significant difference between the average measured SCOD and ffCOD concentrations in the AD WAS. This difference, calculated to be $88 \mathrm{mg} / \mathrm{L}$, was the colloidal COD concentration and it comprised $4 \pm 1 \%$ of the TCOD of the AD WAS. As reported in section 4.2.3, the BR WAS did not contain significant concentrations of colloidal COD hence it would appear that a small amount was generated in the AD during Phase 1. The pretreated BR WAS contained an average colloidal COD concentration of $644 \pm 285 \mathrm{mg} / \mathrm{L}$ hence most of this was removed in the AD during Phase 2.

Based on the average measured ffCOD concentrations in the BR and AD WAS, it would appear that $\mathrm{S}_{\mathrm{us}}$ was generated in the digester in addition to that generated in the BR. The average ffCOD concentration in the AD WAS was $133 \mathrm{mg} / \mathrm{L}$ higher than that in the BR WAS and a t-test at the $95 \%$ confidence level showed that this difference was significant. This difference was attributed to additional $S_{u s}$ that was generated in the digester which suggested that the $S_{u s}$ was generated by endogenous decay rather than by growth. Similar to the $S_{\text {us }}$ generated in the BR, the $S_{\text {us }}$ generated in the AD was probably due to the production of SMPs. Many studies have shown that the majority of soluble organic matter in WWTP effluent is of microbial origin (Namkung and Rittmann, 1986). A portion of the SMPs generated in the AD may have been due to the decay of bacteria that stored 
COD. For example, $S_{\text {us }}$ generation upon the decay of polyphosphate accumulating organisms (PAOs) has been included in the BioWin Integrated Model®.

Figure 4.12 shows that switching from raw to pretreated feed did not alter the VSS concentration of the AD WAS. The average VSS concentration of the AD WAS was $1667 \pm 117 \mathrm{mg} / \mathrm{L}$ in Phase 1 and $1557 \pm 90 \mathrm{mg} / \mathrm{L}$ in Phase 2 and a t-test at the $95 \%$ confidence level revealed no significant difference between these two average values. This indicates that pretreatment did not change the fraction of the BR WAS that could be biodegraded by the AD biomass, despite the fact that the organics in the pretreated WAS were substantially more solubilized than the organics in the raw WAS. With the HRT employed in the AD it was expected that the VSS concentration in the AD would have decreased had pretreatment increased the extent of aerobic biodegradability of the digester feed.

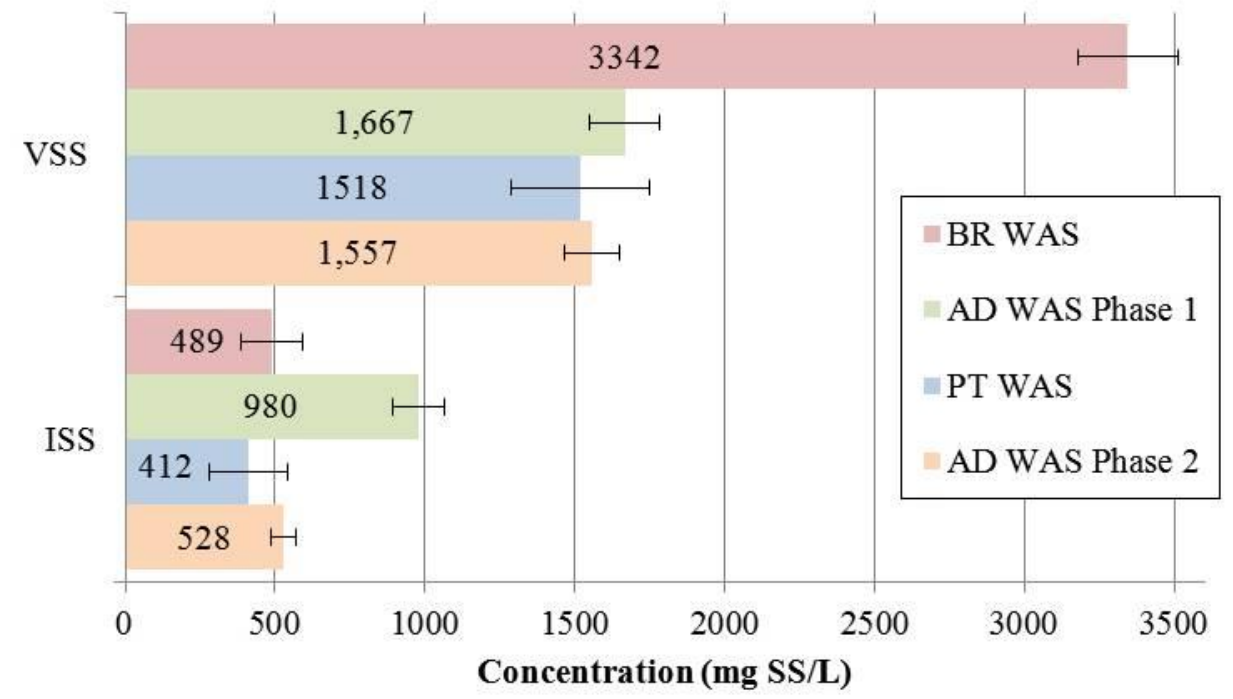

Figure 4.12 Average SS Concentrations in Sludge Streams in Phases 1 and 2

The VSS removal in the AD was calculated according to equation 4.10. The average VSS removal in the $\mathrm{AD}$ was $50 \pm 7 \%$ in Phase 1 . According to Tchobanoglous et al. (2003), VSS reductions ranging from 35 to $50 \%$ are achievable by aerobic digestion with an SRT of $40 \mathrm{~d}$ at $20^{\circ} \mathrm{C}$. Thus the VSS reduction in the Phase $1 \mathrm{AD}$ was at the upper end of the reported range despite the fact that the SRT was only $10 \mathrm{~d}$. It is important to note that the reported range was for aerobic digesters treating WAS from WWTPs with primary treatment where 20 to $35 \%$ of the WAS is assumed to be nonbiodegradable. The high observed VSS reduction in the Phase 1 AD was likely due to two reasons. First, the non-biodegradable fraction of the WAS was expected to be lower than typical because the system in this study was fed with synthetic wastewater that did not contain $X_{i}$. Therefore, the non- 
biodegradable particulate COD fraction of both the BR and AD WAS consisted only of $Z_{\mathrm{e}}$. Second, a substantial fraction of stored COD was present in the BR WAS and this was likely more biodegradable than $\mathrm{Z}_{\mathrm{bh}}$.

$$
\text { VSS removal } \%=\frac{V S S_{A D F e e d}-V S S_{A D W A S}}{V S S_{A D \text { Feed }}}
$$

Using equation 4.10, it was shown that VSS removal did not occur in the AD in Phase 2. A t-test at the $95 \%$ confidence level showed that the average VSS concentration of the pretreated BR WAS was equivalent to that of the Phase 2 AD WAS. Thus the digester did not remove VSS beyond that which had been removed by pretreatment. This was expected because pretreatment substantially solubilized organic matter, as was shown in section 4.2.3.

Although the TCOD, SCOD, ffCOD and VSS concentrations remained relatively stable in the AD during Phases 1 and 2, the average ISS concentration decreased 46\% in Phase 2, as shown in Figure 4.12. A t-test at the $95 \%$ confidence level showed that this decrease was significant. Ramdani et al. (2012) showed that the ISS/TSS ratio for active heterotrophs is almost four times greater than that for endogenous decay products. Thus the measured ISS concentration may have been lower in the AD in Phase 2 than in Phase 1 because the active fraction in the AD was lower in Phase 2. As will be presented in section 4.3.2.3, the active fraction in the $\mathrm{AD}$ determined from the offline respirometric data was $55 \pm 13 \%$ in Phase 1 and $32 \pm 7 \%$ in Phase 2 . The difference between these two averages was $23 \%$ and a t-test at the $95 \%$ confidence level showed that this difference was significant.

In summary, the results presented in this section suggested that the AD WAS was entirely comprised of $Z_{\mathrm{bh}}$ and $Z_{\mathrm{e}}$ in Phases 1 and 2. It appeared that any $\mathrm{X}_{\mathrm{Sto}}$ present in the WAS fed to the AD was depleted in the AD. The VSS concentration in the AD remained constant during Phases 1 and 2 which indicated that pretreatment did not alter the biodegradable fraction of the BR WAS. Furthermore, the ffCOD concentration in the AD remained constant during Phases 1 and 2 which suggested that pretreatment did not generate $S_{\text {us }}$. These findings will be used in section 4.3.2.4 and chapter 5 to help fractionate the COD of the raw and pretreated BR WAS and AD WAS. 


\subsection{Biological Analyses of Process Streams}

\subsubsection{Online Respirometry}

In the online respirometry in the $\mathrm{AD}$, automatic aeration control was used to allow the $\mathrm{DO}$ concentration to fluctuate between 3 and $5 \mathrm{ppm}$, as described in section 3.6.1. A typical DO concentration profile measured in the AD over 3 days is shown in Figure 4.13. The rate of DO decline was calculated for every time period between the high and low DO set points to generate OUR values.

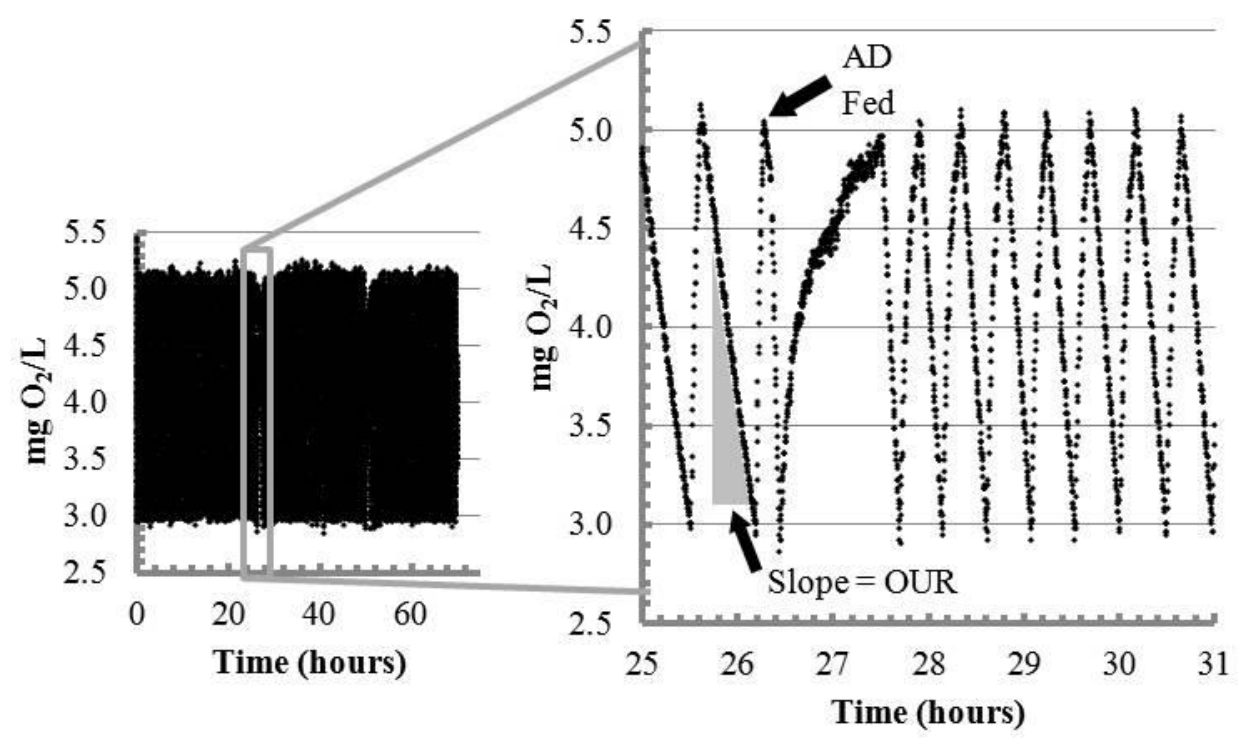

Figure 4.13 Typical DO Concentration in AD and OUR Calculation from Rate of DO Decline

The OUR values were plotted with respect to time as shown in Figure 4.14. Three complete reaction periods are visible in Figure 4.14. The highest OUR values occurred immediately following substrate addition when the substrate utilization rate in the digester was highest. It can also be observed that the OUR values became effectively constant at the end of each reaction period, implying that most of the substrate was consumed. The area under the OUR curve during the entire reaction period was calculated as the cumulative oxygen uptake $\left(\Sigma \mathrm{OU}_{\mathrm{t}}\right)$ in the reactor. The measured OU was entirely carbonaceous because the nitrogenous OU was suppressed throughout Phases 1 and 2. Since the length of the reaction periods varied by 2 to 3 hours between days, the average $\Sigma O U_{t}$ per reaction period was determined over the three consecutive days of the online respirometry test. 


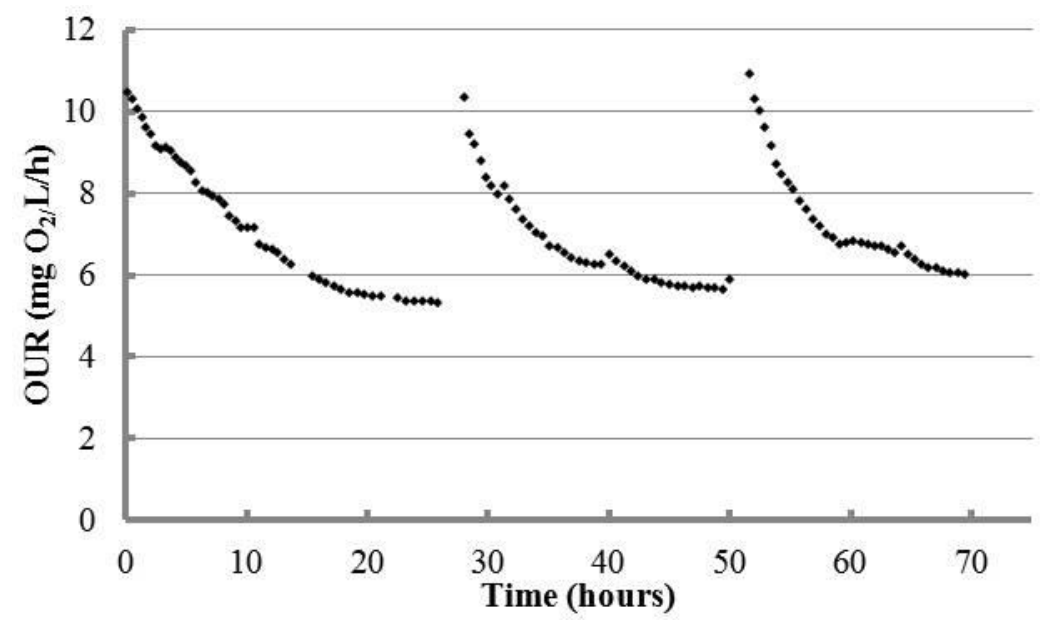

Figure 4.14 Typical OUR in AD

Only one successful online respirometry measurement was made during Phase 1 whereas 12 successful measurements were obtained during Phase 2. The limited number of measurements in phase 1 was due to challenges associated with the experimental apparatus. The Jenco@ model LD900-5-DO Industrial Inline DO Probe used in this project consisted of a gold cathode and silver anode submersed in an electrolyte solution. Oxygen entered the probe through a permeable membrane by diffusion and was reduced at the cathode, creating a measurable electrical current. The Bodine ${ }^{\circledR}$ Model 0158 DC Gearmotor used to mix the AD contents imparted a current to the liquid via the shaft and this interfered with the DO measurement and control. Several unsuccessful attempts were made to isolate the DC gearmotor. Hence, accurate $\Sigma \mathrm{OU}_{\mathrm{t}}$ measurements were only obtained when this DC gearmotor was replaced by an AC motor.

In addition, it was during Phase 1 that it was observed that the anode and cathode of the DO probe underwent rapid and extensive fouling while the probe was immersed in the AD and this negatively impacted the DO readings. This fouling may have been caused by the relatively high chloride concentration in the $\mathrm{AD}$, originating from the ammonium chloride, ferric chloride, magnesium chloride and calcium chloride used in the synthetic wastewater. A special reconditioning kit was ordered from the supplier and used after every respirometry run to restore the gold cathode. The silver anode was cleaned after every test by soaking the probe overnight in $3 \%$ ammonium hydroxide and then sanding it to remove the tarnish. Accurate $\Sigma \mathrm{OU}_{\mathrm{t}}$ measurements were only obtained when these cleaning procedures were carefully followed prior to use. 
The $\Sigma \mathrm{OU}_{\mathrm{t}}$ in the $\mathrm{AD}$ over the reaction period was assumed to be comprised of two parts: the $\Sigma \mathrm{OU}$ due to the endogenous respiration of the AD biomass $\left(\Sigma \mathrm{OU}_{\mathrm{e}}\right)$ and the $\Sigma \mathrm{OU}$ attributed to the WAS fed to the $\mathrm{AD}\left(\Sigma \mathrm{OU}_{\mathrm{s}}\right)$. The $\Sigma \mathrm{OU}_{\mathrm{s}}$ is discussed further here. In Phase 1, the COD of the BR WAS fed to the $\mathrm{AD}$ was expected to be essentially comprised of $\mathrm{Z}_{\mathrm{bh}}, \mathrm{Z}_{\mathrm{e}}$ and $\mathrm{X}_{\mathrm{STO}}$. Therefore, the $\Sigma O \mathrm{U}_{\mathrm{s}}$ was expected to result from growth on $\mathrm{X}_{\text {STO }}$ and decay of $\mathrm{Z}_{\mathrm{bh}}$. In Phase 2, the COD of the pretreated BR WAS was expected to be comprised of $S_{\mathrm{bsc}}, \mathrm{X}_{\mathrm{sp}}$ and $\mathrm{Z}_{\mathrm{e}}$. It was hypothesized that pretreatment would not change $Z_{\mathrm{e}}$ but would convert $\mathrm{X}_{\mathrm{STO}}$ to $\mathrm{S}_{\mathrm{bsc}}$ and $\mathrm{Z}_{\mathrm{bh}}$ to portions of $\mathrm{S}_{\mathrm{bsc}}$ and $\mathrm{X}_{\mathrm{sp}}$. Therefore the $\Sigma O \mathrm{U}_{\mathrm{s}}$ in Phase 2 was expected to result from growth on $\mathrm{S}_{\mathrm{bsc}}$ and $\mathrm{X}_{\mathrm{sp}}$ and decay of $\mathrm{Z}_{\mathrm{bh}}$.

An analysis of the COD and VSS measurements in the AD showed that the average food to microorganism $(\mathrm{F} / \mathrm{M})$ ratio was $0.30 \pm 0.05 \mathrm{mgCOD} / \mathrm{mgVSS}$ throughout Phases 1 and 2 . This was low enough to cause a decay-dominated OUR response (Musser, 2009). Therefore, it was assumed that any growth OUR response would be masked by the decay response. This assumption was validated by the results. The OUR response in the AD shown in Figure 4.14 was measured during Phase 2. However, these curves were typical of the OUR measured in the AD throughout Phases 1 and 2. As shown in Figure 4.14, the OUR decreased over the reaction period. The shape of these decreasing OUR curves shows substrate depletion and endogenous respiration but not growth.

To determine the $\Sigma \mathrm{OU}_{\mathrm{s}}$ value $\left(\mathrm{mg} \mathrm{O}_{2} / \mathrm{L}\right.$ ) for each online respirometric test, the $\Sigma \mathrm{OU}_{\mathrm{e}}$ value was first estimated from the offline respirometric tests on the AD WAS. The endogenous OUR equation 2.4 proposed by Jones et al. (2009) was modified to eliminate the nitrogenous OUR, producing equation 4.11.

$$
\text { OUR }=1-f\left(b_{h}\right) Z_{b h 0} e^{-\left(b_{h}\right) t}
$$

A nonlinear regression fit of equation 4.11 to the endogenous OUR response measured by offline respirometry was used to yield the initial active heterotroph concentration in the AD WAS, as described previously in section 2.1.5.1. Offline respirometry was carried out at $25^{\circ} \mathrm{C}$ hence the value of $b_{h, 25 C}$ was determined using equation 4.12. The assumed value of $b_{h, 20 C}$ was $0.24 \mathrm{~d}^{-1}$ (Henze et al., 2008).

$$
b_{h, T}=b_{h, 20 C}(1.029)^{T-20}
$$

Using equation 4.12 , the value of $b_{h, 25 c}$ was calculated to be $0.28 \mathrm{~d}^{-1}$. The results of the offline respirometric tests will be presented in the next section however the measured and predicted endogenous OUR $\left(\mathrm{OUR}_{\mathrm{e}}\right)$ curves obtained on day 264 are shown as an example in Figure 4.15. This curve fit was representative of those obtained for the other measurement events. 


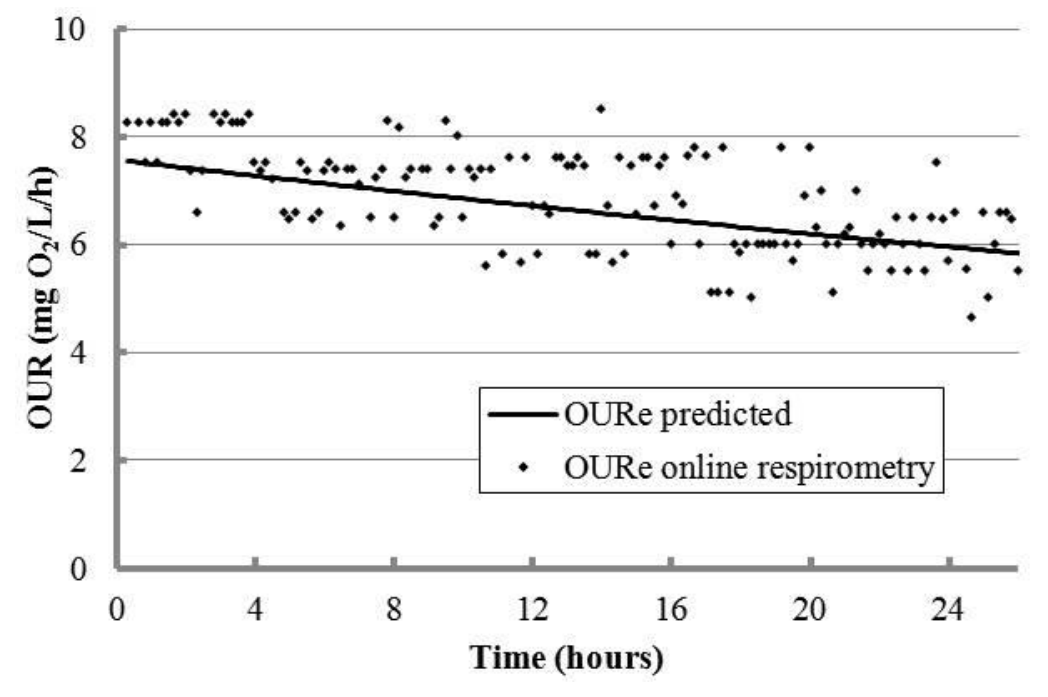

Figure 4.15 Measured and Predicted Endogenous OUR in AD WAS at $25^{\circ} \mathrm{C}$

Using the predicted initial active heterotroph concentration, equation 4.11 with $\mathrm{b}_{\mathrm{h}, 20 \mathrm{C}}$ was then used to estimate the endogenous OUR response at $20^{\circ} \mathrm{C}$ in the $\mathrm{AD}$ since online respirometry was carried out at $20^{\circ} \mathrm{C}$. This endogenous OUR response was then plotted together with the total OUR response measured by online respirometry on day 264, as shown in Figure 4.16. These curves are representative of those obtained for the other measurement events. The area under this endogenous OUR curve was then used to estimate the $\Sigma \mathrm{OU}_{\mathrm{e}}$ value which was then subtracted from the area under the total OUR curve $\left(\Sigma \mathrm{OU}_{\mathrm{t}}\right)$ to yield the $\Sigma \mathrm{OU}_{\mathrm{s}}$ value.

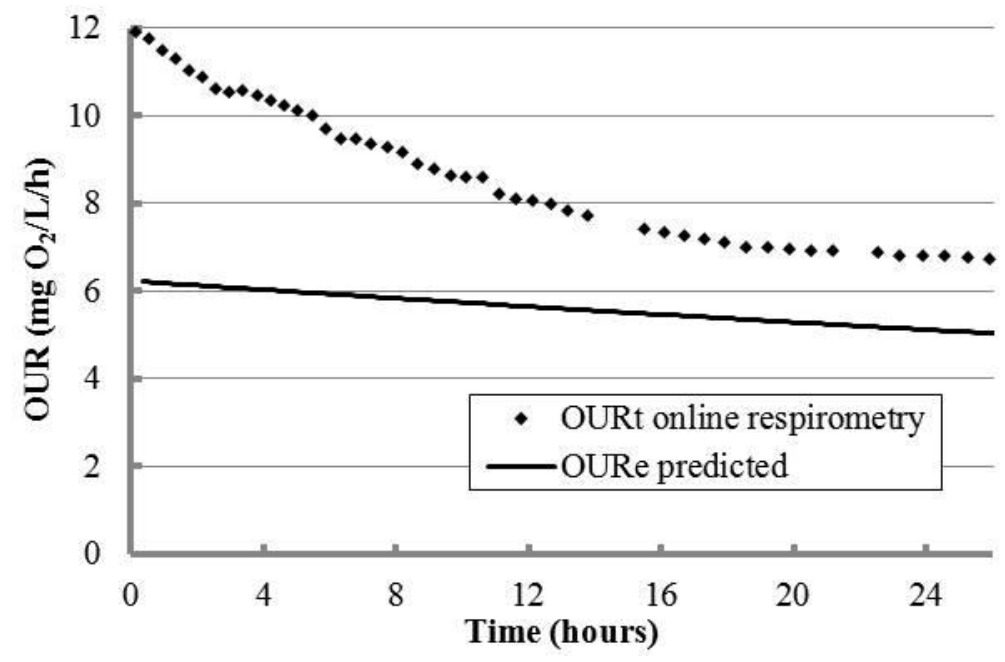

Figure 4.16 Total and Endogenous OUR in $\mathrm{AD}$ at $20^{\circ} \mathrm{C}$ 
The online respirometric data was used to determine whether pretreatment changed the overall aerobic degradability of the BR WAS. The $\Sigma O U_{s}$ value was divided by the measured TCOD concentration of the substrate, i.e. the WAS fed to the AD, for every successful test result. Calculation of the $\Sigma O U_{s} / T C O D$ ratio was based upon the mass associated with each response for one cycle of operation. As mentioned in section 3.3.2, 0.6 L of BR WAS was fed to the AD each cycle and the $\mathrm{AD}$ was $6 \mathrm{~L}$. Thus the calculated $\Sigma \mathrm{OU}_{\mathrm{s}}$ values $\left(\mathrm{mg} \mathrm{O}_{2} / \mathrm{L}\right)$ were multiplied by $6 \mathrm{~L}$ and the corresponding measured TCOD concentrations $(\mathrm{mg} \mathrm{COD} / \mathrm{L})$ of the substrate were multiplied by 0.6 L.

The average value of the $\Sigma \mathrm{OU}_{\mathrm{s}} / \mathrm{TCOD}$ ratio was calculated for Phases 1 and 2. A significant increase in these values between Phase 1 and 2 average would indicate that pretreatment changed the overall aerobic biodegradability of the WAS. The average value of the $\Sigma \mathrm{OU}_{\mathrm{s}} / \mathrm{TCOD}$ ratio was calculated to be $16 \%$ in Phase 1 and $15 \pm 4 \%$ in Phase 2. A t-test at the $95 \%$ confidence level showed that the difference between these two averages was insignificant which suggested that pretreatment did not change the aerobic degradability of the WAS over the AD reaction period.

\subsubsection{Offline Respirometry}

\subsubsection{Shape and Magnitude of OUR Curves}

Unlike online respirometry, it was expected that the OUR curves generated from offline respirometry would show growth responses. This was because the F/M ratio in the offline respirometric tests on bottles containing AD WAS and raw or pretreated BR WAS ranged from 5 to $10 \mathrm{mgCOD} / \mathrm{mgVSS}$ which was sufficiently high to show a growth response if substrate were present in the bottle.

In Phase 1 it was assumed that the BR WAS consisted of $Z_{b h}, Z_{e}$ and $X_{S T O}$ hence the OUR response in bottles containing whole BR WAS was expected to reflect growth on $\mathrm{X}_{\mathrm{STO}}$, depletion of $\mathrm{X}_{\mathrm{STO}}$ and decay of $\mathrm{Z}_{\mathrm{bh}}$. The SRT in the BR was 5 days whereas it was 10 days in the AD hence the BR WAS was expected to have a higher ratio of active organisms to endogenous decay products as compared to the AD. Correspondingly, the OUR $\left(\mathrm{mg} \mathrm{O}_{2} / \mathrm{L} / \mathrm{h}\right)$ in the BR WAS was expected to be greater than that in the AD WAS at any given time during the offline respirometric test. 
Three offline respirometric tests were run during the first phase of the project, from day 179 to 203 , with the bottles containing BR WAS only, AD WAS only and BR WAS that was inoculated with AD WAS. Only the second and third tests produced useful data as residual rbCOD from the synthetic wastewater was present in the first test that was run on day 175. This test was conducted just before the synthetic feed concentration was halved. Figure 4.17 shows a typical OUR response in the bottles of the two successful tests.

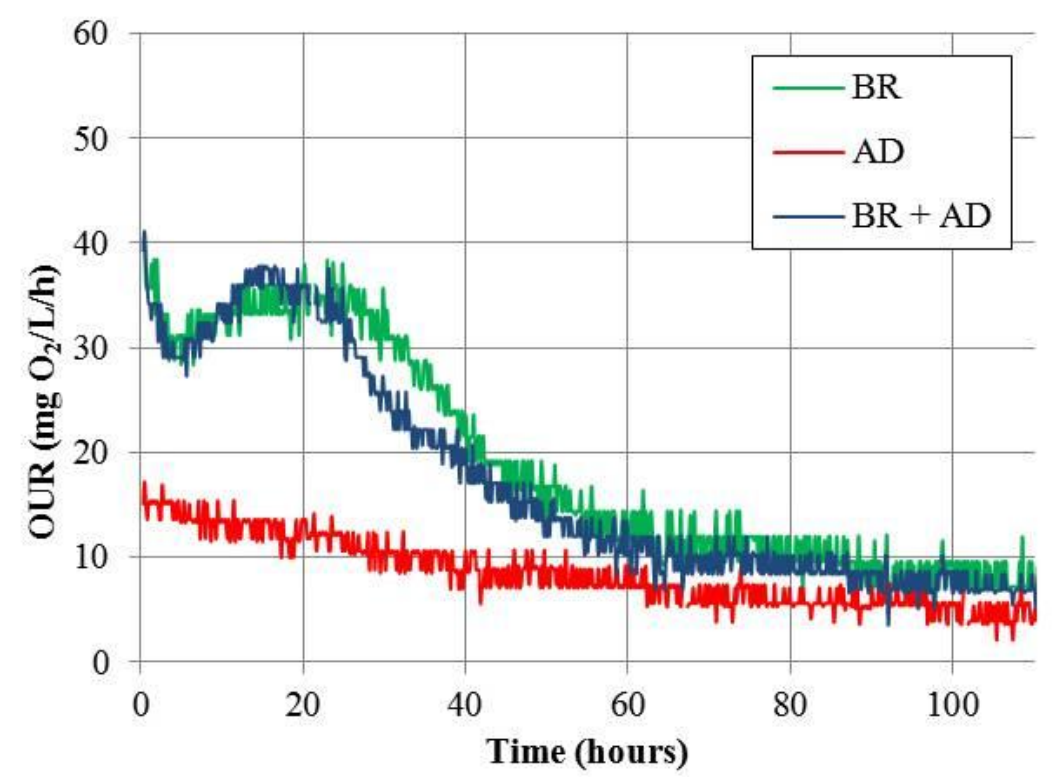

Figure 4.17 Typical Phase 1 OUR in BR WAS, AD WAS and Inoculated BR WAS

In the bottle containing AD WAS, the OUR decreased exponentially with time. This was the expected response for a sludge that consumes oxygen through endogenous respiration alone. In the bottles containing BR WAS only and inoculated BR WAS, the OUR decreased for the first 5 hours of the test, then increased, peaked and finally decreased exponentially with time. The magnitude of these two curves during the first 5 hours was more than twice that of the AD WAS curve. This was expected because the BR was assumed to contain a higher active fraction than the AD. The peak in the OUR curves for BR WAS and inoculated BR WAS suggested biomass growth. Furthermore, because the peaks were observed in these two responses but absent in the AD WAS response, the substrate for this biomass growth was assumed to be present in the BR WAS alone. As previously mentioned, the BR WAS used in the offline respirometry test was taken from the BR at the end of the reaction period, long after the synthetic substrate had been consumed. Hence the apparent growth was not due to the synthetic substrate. The observed OUR responses were therefore consistent with the previously stated hypothesis that the BR WAS contained $\mathrm{X}_{\mathrm{STO}}$ in addition to $\mathrm{Z}_{\mathrm{bh}}$ and $\mathrm{Z}_{\mathrm{e}}$. This hypothesis was supported by the previously described low average COD/VSS ratio of 1.25 in the BR 
WAS; a low average pON/PCOD ratio of 0.05 in the BR WAS; and the observed generation of $\mathrm{S}_{\text {us }}$ in the AD. It appeared that $\mathrm{X}_{\mathrm{STO}}$ was consumed during the batch respirometry and exerted an oxygen demand that was different than that which would be attributed to endogenous respiration.

In Phase 2, offline respirometric tests were run on bottles containing pretreated BR WAS only, AD WAS only and pretreated BR WAS inoculated with AD WAS. Once the feed to the AD was switched from raw to pretreated BR WAS on day 203, one offline respirometric test was carried out every week for 9 weeks. Due to power failures, two of these tests did not run until the endogenous OUR response was clearly visible and thus the data could not be used. Three tests were run when the AD WAS was not acclimatized to the pretreated BR WAS. Only one of these three tests produced usable data as there were various sources of error in the other tests. These error sources included restricted oxygen flow through the tubing and needles, leaks in the bottle caps and water collection in the oxygen supply lines. The set of OUR curves generated in the successful offline respirometric tests on pretreated BR WAS and the associated AD WAS are presented in Appendix C.

Overall, there was no distinguishable difference in the shape of the OUR curves from the inoculated bottles in either the non-acclimatized test or the progressively acclimatized tests. The differences in the areas under these curves will be discussed in section 4.3.2.2.

In Phase 2 it was expected that the OUR response in the bottles containing pretreated BR WAS and AD WAS would result from both growth and decay since pretreatment was expected to convert portions of the TCOD of the BR WAS to $S_{\mathrm{bsc}}$ and $\mathrm{X}_{\mathrm{sp}}$. As shown in section 4.2.3, pretreatment increased the SCOD concentration by $56 \%$. Since it was also shown in section 4.2 .4 that pretreatment did not generate $S_{\mathrm{us}}$, the SCOD generated by pretreatment was expected to be $S_{\mathrm{bsc}}$. Similar to Phase 1, the OUR responses in bottles containing AD WAS were expected to show only decay.

Figure 4.18 shows typical OUR responses in the bottles during Phase 2 of the project. The labels PT, $\mathrm{AD}$ and $\mathrm{PT}+\mathrm{AD}$ represent the respective OUR curves for pretreated BR WAS, AD WAS and pretreated BR WAS inoculated with AD WAS. This graph is representative of the eight successful offline respirometric tests involving pretreated BR WAS and the associated AD WAS. In the bottles containing only AD WAS, the OUR decreased exponentially with time as expected. At any given time during the respirometry test, the height of this curve was always less than that of the Phase $1 \mathrm{AD}$ WAS, indicating that the active fraction was lower in the AD in Phase 2 than in Phase 1 . The bottles containing only pretreated BR WAS exerted oxygen uptake, indicating viable cells remained in the 
sludge after pretreatment. Estimates of the heterotroph concentrations will be presented in section 4.3.2.3. The OUR curve for pretreated BR WAS showed a peak between 60 and 70 hours, suggesting delayed biomass growth. However, the area under this peak comprised only 5\% of the total area under the curve. Therefore, this delayed peak was considered negligible.

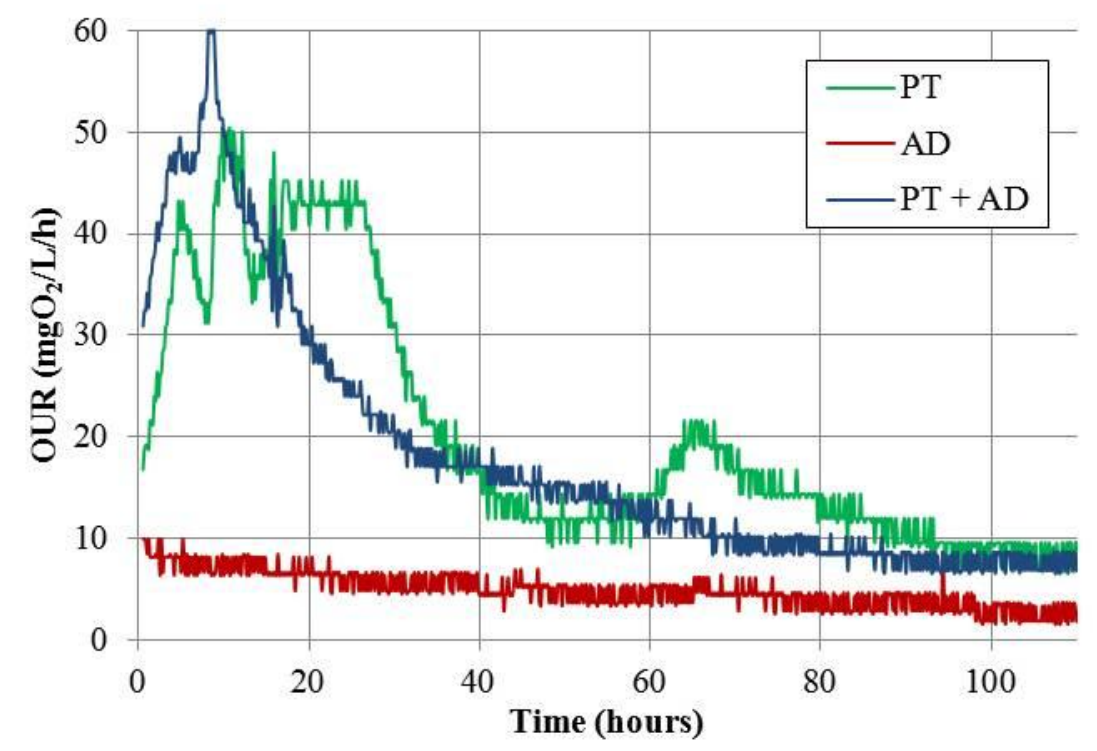

Figure 4.18 Typical Phase 2 OUR in Pretreated BR WAS, AD WAS and Inoculated Pretreated BR WAS

Comparing Figures 4.17 and 4.18, the OUR curve for the inoculated pretreated BR WAS peaked earlier and to a higher value than the OUR curve for the inoculated raw BR WAS. This response is characteristic of growth on rbCOD.

For each successful respirometry run, the mass OUR curves $\left(\mathrm{mg} \mathrm{O}_{2} / \mathrm{h}\right)$ were plotted by multiplying the measured OUR concentration $\left(\mathrm{mg} \mathrm{O}_{2} / \mathrm{L} / \mathrm{h}\right)$ by the volume of WAS in the bottle. The endogenous OUR curve was subtracted from the OUR curve of the inoculated bottle to yield the OUR response of the substrate only. This approach was carried out for both the whole and filtered substrate. The substrate OUR responses from the offline respirometric tests on pretreated BR WAS and associated AD WAS were compared to the substrate OUR responses from the tests on BR WAS and associated AD WAS. The OUR responses of the whole and filtered pretreated BR WAS substrates were found to be reproducible as were those of the whole and filtered BR WAS substrates. Therefore, the OUR responses of the whole and filtered pretreated BR WAS substrates (Phase 2) were compared to the OUR responses of the whole and filtered BR WAS substrates (Phase 1), as shown in Figure 4.19. 


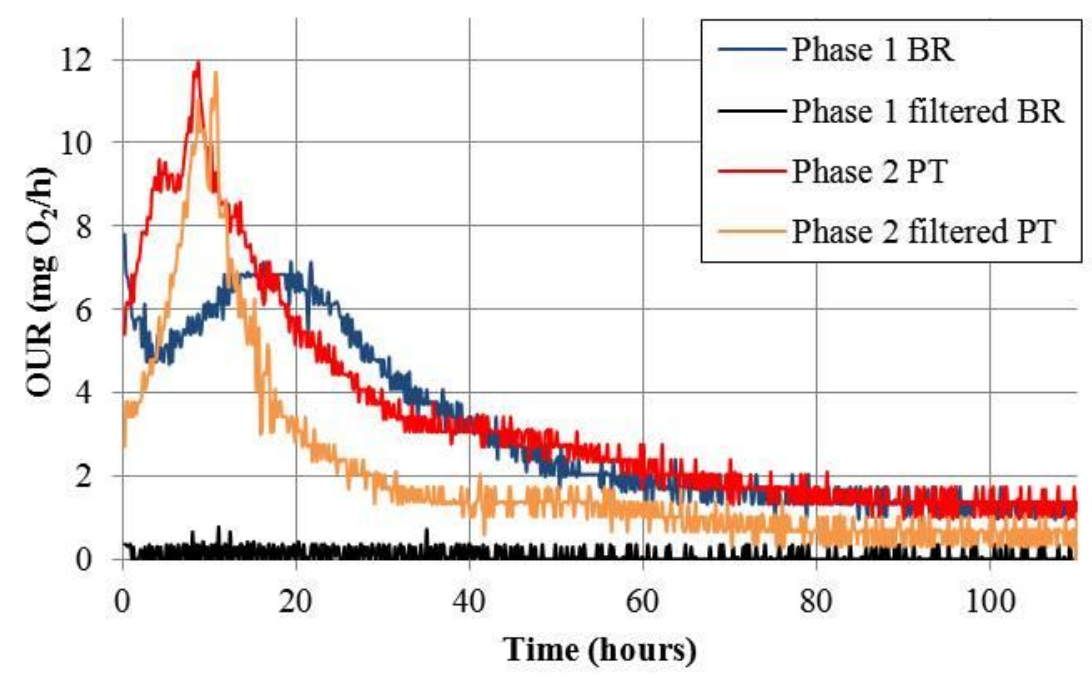

Figure 4.19 Comparison of Typical Substrate OUR in Phases 1 and 2

From Figure 4.19 it can be seen that the OUR in the filtered BR WAS was negligible, indicating the absence of soluble biodegradable COD. The biodegradable COD of the BR WAS was thus entirely particulate. This was expected because filtration removed the only two components in the BR WAS that were assumed to exert an oxygen demand, i.e. $\mathrm{Z}_{\mathrm{bh}}$ and $\mathrm{X}_{\mathrm{STO}}$. In contrast, the area under the OUR curve for the filtered pretreated WAS contributed more than $60 \%$ of the area under the whole pretreated WAS curve which demonstrates that pretreatment substantially solubilized biodegradable COD.

\subsubsection{Extent of Aerobic Biodegradability}

Similar to online respirometry, offline respirometry was used to determine whether pretreatment changed the overall aerobic degradability of the BR WAS. The cumulative oxygen uptake $\left(\mathrm{mg} \mathrm{O}_{2}\right)$ associated with the substrate during every successful offline respirometric test was divided by the measured TCOD (mg COD) of the substrate at the beginning of the test. The average value of this ratio was calculated for the raw BR WAS and pretreated BR WAS. A significant difference between these average ratios would indicate that pretreatment changed the overall aerobic biodegradability of the WAS. This ratio was only determined for the whole substrate because it was shown that the SCOD concentration in the BR WAS was negligible. Hence the cumulative oxygen uptake and TCOD of the filtered BR WAS was negligible. 
The cumulative oxygen uptake $\left(\mathrm{mg} \mathrm{O}_{2}\right)$ associated with the substrate was calculated in two separate ways using the offline respirometric data. The first approach relied on the measured mass of TCOD in the respirometry bottle at the beginning and end of the test whereas the second approach employed the mass of gas phase oxygen measured by the respirometer during the test.

In the first approach, the difference between the initial and final mass of TCOD in each bottle $\left(\mathrm{TCOD}_{\mathrm{i}}-\mathrm{TCOD}_{\mathrm{f}}\right)$ was calculated for each offline respirometric test. The mass of TCOD in the bottle was determined by multiplying the measured TCOD concentration (mg COD/L) in the bottle by the volume of liquid in the bottle. The $\left(\mathrm{TCOD}_{\mathrm{i}}-\mathrm{TCOD}_{\mathrm{f}}\right)$ value for the substrate was then determined by subtracting the $\left(\mathrm{TCOD}_{\mathrm{i}}-\mathrm{TCOD}_{\mathrm{f}}\right)$ value in the bottle containing only AD WAS from the $\left(\mathrm{TCOD}_{\mathrm{i}}-\right.$ $\mathrm{TCOD}_{\mathrm{f}}$ ) value in the bottle containing AD WAS and raw or pretreated BR WAS.

The biodegradability of the substrate was then assessed by dividing the $\left(\mathrm{TCOD}_{\mathrm{i}}-\mathrm{TCOD}_{\mathrm{f}}\right)$ value for the substrate by the initial TCOD of the substrate in the bottle for each respirometry run, as shown in Figure 4.20. A significant difference between the average $\left(\mathrm{TCOD}_{\mathrm{i}}-\mathrm{TCOD}_{\mathrm{f}}\right) / \mathrm{TCOD}_{\mathrm{i}}$ ratios for raw BR WAS and pretreated BR WAS would indicate that pretreatment changed the overall aerobic biodegradability of the WAS. Because the BR WAS was shown to remain stable throughout Phases 1 and 2 , the only factor that could cause a change in the overall aerobic biodegradability of the BR WAS would be pretreatment.

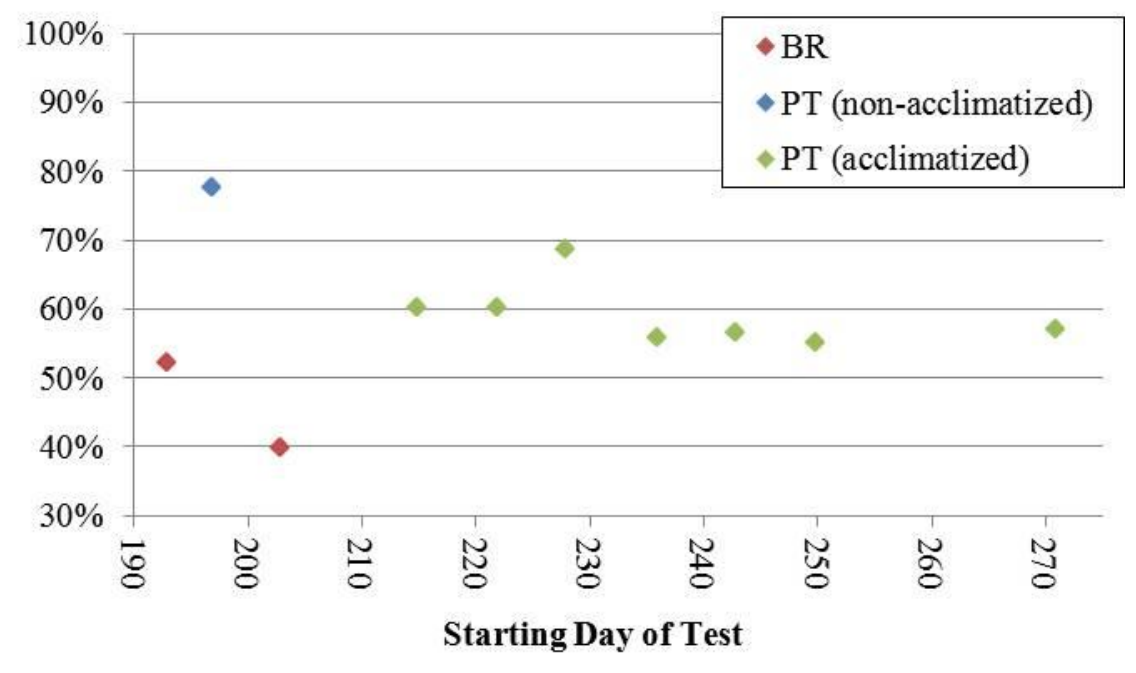

Figure 4.20 $\left(\mathrm{TCOD}_{\mathrm{i}}-\mathrm{TCOD}_{\mathrm{f}}\right) / \mathrm{TCOD}_{\mathrm{i}}$ Ratio from Offline Respirometry on Substrate

Using the data presented in Figure 4.20, the average $\left(\mathrm{TCOD}_{\mathrm{i}}-\mathrm{TCOD}_{\mathrm{f}}\right) / \mathrm{TCOD}_{\mathrm{i}}$ values were $46 \pm 15 \%$ for the BR WAS, $78 \%$ for the pretreated BR WAS with non-acclimatized inoculum and $59 \pm 11 \%$ for 
the pretreated BR WAS with acclimatized inoculum. A t-test at the 95\% confidence level showed that the average $\left(\mathrm{TCOD}_{\mathrm{i}}-\mathrm{TCOD}_{\mathrm{f}}\right) / \mathrm{TCOD}_{\mathrm{i}}$ ratio for BR WAS was equivalent to the average ratio for pretreated BR WAS with acclimatized inoculum, indicating that pretreatment did not change the fraction of the BR WAS that could be degraded by acclimatized inoculum. This finding corroborated the results of the online respirometric tests and supported the finding that the VSS concentration in the $\mathrm{AD}$ remained constant during Phases 1 and 2. Considering the seven data points for the acclimatized pretreated $\left(\mathrm{TCOD}_{\mathrm{i}}-\mathrm{TCOD}_{\mathrm{f}}\right) / \mathrm{TCOD}_{\mathrm{i}}$ ratio in Figure 4.20, the last four data points had an average and standard deviation of $56 \pm 1 \%$, which showed very low variability. The stability of these last four measurements indicated that the AD biomass had acclimatized to the pretreated feed.

Based on the data presented in Figure 4.20, the $\left(\mathrm{TCOD}_{\mathrm{i}}-\mathrm{TCOD}_{\mathrm{f}}\right) / \mathrm{TCOD}_{\mathrm{i}}$ ratio for pretreated $\mathrm{BR}$ WAS with non-acclimatized inoculum was $32 \%$ higher than the average ratio for BR WAS and a ttest at the 95\% confidence level showed that this difference was significant. This suggested that pretreatment increased the fraction of the BR WAS that could be degraded by non-acclimatized inoculum. However, because there was only one valid measurement of pretreated BR WAS with non-acclimatized inoculum, additional experiments should be carried out to further investigate this trend. By comparison, Kianmehr (2010) found that with WAS pretreated with ozone, the biodegradable fraction determined by offline respirometry was higher using an acclimatized inoculum than a non-acclimatized inoculum. However, Kianmehr (2010) also demonstrated that it was unnecessary to acclimatize the inoculum when using sonication pretreatment. Further studies should be carried out to determine the impact of biomass adaptation on the degradation of WAS pretreated by HPTH.

The second approach to estimate the aerobic biodegradability from the offline respirometry employed gas phase oxygen consumption to estimate the cumulative oxygen uptake $\left(\mathrm{mg} \mathrm{O}_{2}\right)$ associated with the substrate. In these cases, the $\Sigma \mathrm{OU}_{\mathrm{s}}$ value $\left(\mathrm{mg} \mathrm{O}_{2}\right)$ for each offline respirometric test was determined by subtracting the measured $\Sigma \mathrm{OU}$ in the sample bottles containing only AD WAS $\left(\Sigma \mathrm{OU}_{\mathrm{e}}\right)$ from the $\Sigma \mathrm{OU}$ value in the inoculated bottle $\left(\Sigma \mathrm{OU}_{\mathrm{t}}\right)$. The $\Sigma \mathrm{OU}_{\mathrm{s}}$ value was then corrected for the mass of oxygen required to reach liquid saturation in the bottle since the DO concentration was effectively zero at the beginning of the respirometry test. In addition, the $\Sigma O U_{s}$ value was corrected for the mass of oxygen manually drawn into the bottle using a syringe in the air-tightness test performed at the start of the run. The biodegradability of the substrate was then assessed by dividing the $\Sigma O U_{s}$ value for the substrate by the measured initial mass of TCOD of the substrate for each respirometry run, as shown in Figure 4.21. 


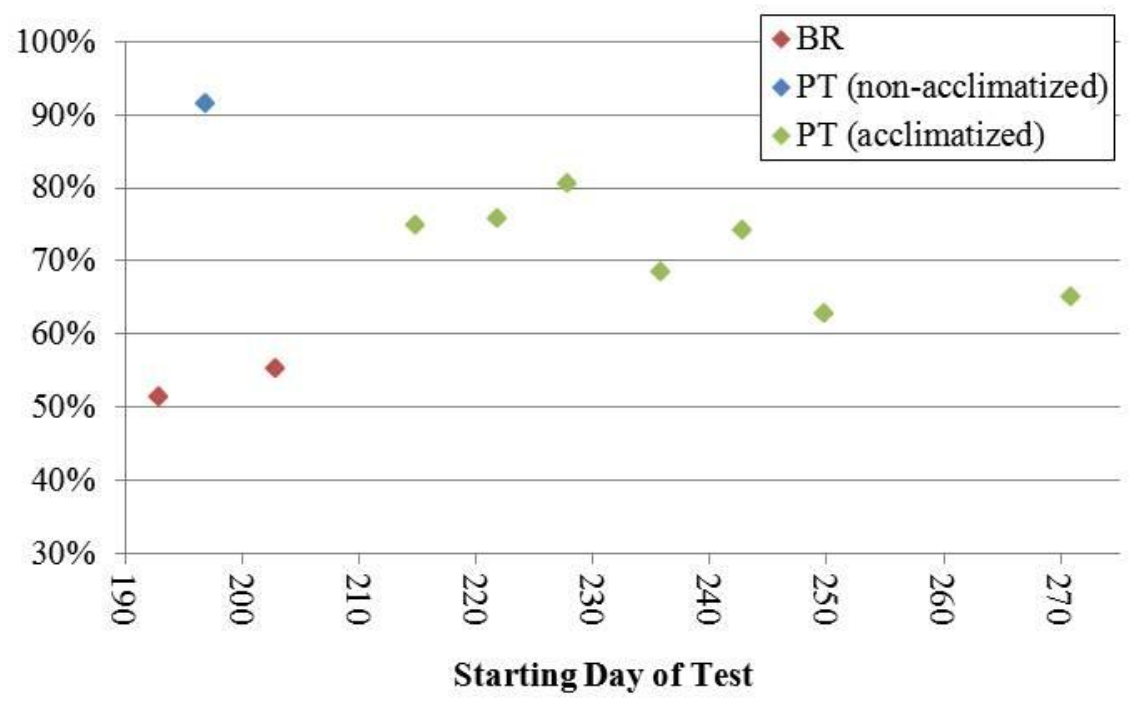

Figure 4.21 $\Sigma \mathrm{OU}_{\mathrm{s}} / \mathrm{TCOD}_{\mathrm{i}}$ Ratio from Offline Respirometry on Substrate

Overall, the trends in Figure 4.21 were similar to those in Figure 4.20 in terms of the relative magnitude of the ratios among the different types of substrate. The average $\Sigma O U_{s} / T_{C O D}$ values were calculated for the BR WAS, pretreated WAS with non-acclimatized inoculum and pretreated WAS with acclimatized inoculum. It was found that each of these average values was higher than the respective average value calculated using the measured initial and final TCOD concentrations in the sample bottle. Because the $\left(\mathrm{TCOD}_{\mathrm{i}}-\mathrm{TCOD}_{\mathrm{f}}\right) / \mathrm{TCOD}_{\mathrm{i}}$ ratio in the first approach relied on only one measurement technique whereas the $\Sigma \mathrm{OU}_{\mathrm{s}} / \mathrm{TCOD}_{\mathrm{i}}$ ratio in the second approach involved two techniques, there may have been a higher level of error associated with the second approach than with the first. The potential sources of error are discussed subsequently.

The accuracy of the TCOD measurements was verified in several ways. Every day the TCOD concentrations in the respirometry bottle were measured, blank and standard samples were also tested. The measured concentrations of the blanks and standards were significantly close to the expected values. In addition, on day 203 and 250, four replicate samples were taken from a randomly selected respirometry bottle and a COD standard was added to half of these replicates. The average measured TCOD of the two replicates containing only sample was subtracted from that of the replicates containing standard and sample in order to calculate the TCOD of the standard. There was no significant difference between the calculated value of the standard and the directly measured value. Therefore, the measured TCOD concentrations in the offline respirometry bottles at the beginning and end of the test were believed to be accurate. 
The TCOD closure error was calculated according to equation 4.13 for the bottles containing AD WAS only, substrate only and inoculated substrate in every successful batch respirometry test. In this equation, the cumulative oxygen uptake $\left(\mathrm{mg} \mathrm{O}_{2}\right)$ in the bottle calculated by the first approach $\left(\mathrm{TCOD}_{\mathrm{i}}\right.$ - $\mathrm{TCOD}_{\mathrm{f}}$ ) was subtracted from that calculated by the second approach that employed the gas phase oxygen consumption ( $\Sigma \mathrm{OU})$. This difference was then divided by the measured mass of TCOD in the bottle at the end of the test $\left(\mathrm{TCOD}_{\mathrm{f}}\right)$ and expressed as a percentage.

$$
\text { TCOD Closure Error } \%=\frac{\Sigma O U-\left(T C O D_{i}-T C O D_{f}\right)}{T C O D_{f}}
$$

The cumulative oxygen uptake calculated on the basis of oxygen consumption was consistently higher than that calculated by COD removal; hence the TCOD closure error was consistently positive. The average TCOD closure error for bottles containing only AD WAS was found to be $5 \pm 2 \%$, which was relatively low. By comparison, the TCOD closure error ranged from 10 to $35 \%$ in the other bottles. As expected, the cumulative oxygen uptake in bottles containing AD WAS was consistently lower than the cumulative oxygen uptake in bottles containing either substrate or inoculated substrate. The higher TCOD closure errors would therefore appear to be associated with the higher oxygen demands. Since the TCOD measurements were believed to be accurate, this positive closure error indicated that the offline respirometric tests overestimated the oxygen uptake in the bottles. As a result, the $\Sigma O \mathrm{OU}_{\mathrm{s}} / \mathrm{TCOD}_{\mathrm{i}}$ ratio was probably less accurate than the $\left(\mathrm{TCOD}_{\mathrm{i}}-\mathrm{TCOD}_{\mathrm{f}}\right) / \mathrm{TCOD}_{\mathrm{i}}$ ratio. Therefore, the assessment of the impact of pretreatment on the overall aerobic biodegradability of the BR WAS was based on the comparison of the average $\left(\mathrm{TCOD}_{\mathrm{i}}-\mathrm{TCOD}_{\mathrm{f}}\right) / \mathrm{TCOD}_{\mathrm{i}}$ ratios rather than a comparison of the average $\Sigma \mathrm{OU}_{\mathrm{s}} / \mathrm{TCOD}_{\mathrm{i}}$ ratios.

As presented in section 4.3.1, the average $\Sigma \mathrm{OU}_{\mathrm{s}} / \mathrm{TCOD}_{\mathrm{i}}$ ratio determined by online respirometry was $16 \%$ for BR WAS and $15 \pm 4 \%$ for pretreated BR WAS. By comparison, the average $\left(\mathrm{TCOD}_{\mathrm{i}}-\right.$ $\mathrm{TCOD}_{\mathrm{f}} / \mathrm{TCOD}_{\mathrm{i}}$ ratio determined by offline respirometry was $46 \pm 15 \%$ for BR WAS and $59 \pm 11 \%$ for pretreated BR WAS with acclimatized inoculum. Thus the average $\left(\mathrm{TCOD}_{\mathrm{i}}-\mathrm{TCOD}_{\mathrm{f}}\right) / \mathrm{TCOD}_{\mathrm{i}}$ ratios determined by offline respirometry for BR WAS and pretreated BR WAS with acclimatized inoculum were $30 \%$ and $44 \%$ higher, respectively, than the corresponding ratios determined by online respirometry. T-tests at the $95 \%$ confidence level showed that both of these differences were significant. It was hypothesized that the online respirometric measurements underestimated the cumulative oxygen uptake over the reaction period because these measurements failed to capture rapid and high oxygen uptake. In Phases 1 and 2, delays of 30 to 60 minutes were observed in raising the DO concentration from the low to high set-points during the period immediately after the AD was fed. These delays were presumably due to insufficient aeration in the AD. In addition, the differing 
methodologies of the two respirometric tests may have further contributed to generating $\left(\mathrm{TCOD}_{\mathrm{i}}-\right.$ $\mathrm{TCOD}_{\mathrm{f}} / \mathrm{TCOD}_{\mathrm{i}}$ ratios from offline respirometry that were significantly higher than the magnitudes of the $\Sigma \mathrm{OU}_{\mathrm{s}} / \mathrm{TCOD}_{\mathrm{i}}$ ratios from online respirometry. The offline respirometric tests were run for 4 to 5 days at an $\mathrm{F} / \mathrm{M}$ ratio of 5 to $10 \mathrm{mgCOD} / \mathrm{mgVSS}$. By contrast, the online respirometric tests measured the $\Sigma \mathrm{OU}$ in the $\mathrm{AD}$ over the reaction period of 22 hours and the average F/M ratio in the $\mathrm{AD}$ was 0.30 $\pm 0.05 \mathrm{mgCOD} / \mathrm{mgVSS}$.

In summary, the offline respirometric results showed that pretreatment did not change the overall aerobic biodegradability of the BR WAS. It was previously shown in section 4.2.3 that pretreatment also did not change the TCOD of the BR WAS. Therefore, it was hypothesized that the concentration of non-biodegradable COD in the pretreated BR WAS was equivalent to that in the raw BR WAS. This non-biodegradable COD was assumed to be comprised of only $Z_{e}$ since the $S_{\text {us }}$ concentration in the BR WAS was negligible and $S_{\text {us }}$ was not generated by pretreatment. Thus the concentration of bCOD in the BR WAS was assumed to be equivalent to that in the pretreated BR WAS. The bCOD of the BR WAS was shown to be comprised of $Z_{\mathrm{bh}}$ and $\mathrm{X}_{\mathrm{STO}}$. It was hypothesized that the bCOD of the pretreated BR WAS was comprised of $S_{\mathrm{bsc}}$ and $\mathrm{X}_{\mathrm{sp}}$. This hypothesis was based on the shape and magnitude of the OUR curves generated by offline respirometry and the measured increase in SCOD concentration caused by pretreatment. It was not possible to use the approach proposed by Kianmehr (2010) to quantify the $\mathrm{S}_{\mathrm{bsc}}$ concentration in the pretreated BR WAS using the offline respirometric data. The F/M ratio was high in the tests employed in the current study hence significant growth occurred in bottles believed to contain $S_{\text {bsc. }}$. The peaks due to growth were high and extended and multiple successive peaks were typically observed. The area under the OUR curve was thus a complex function of cell growth, decay and the oxidation of $S_{\mathrm{bsc}}$ and $X_{\mathrm{sp}}$. Instead, the $S_{\mathrm{bsc}}$ and $X_{\mathrm{sp}}$ concentrations in the pretreated BR WAS were estimated by fitting OUR responses predicted by activated sludge models to the measured offline respirometric data. This approach will be presented in section 5 .

\subsubsection{Active and Endogenous COD Fractions}

This section presents the results of an analysis of the offline respirometric data that estimated the concentrations of $Z_{b h}$ and $Z_{e}$ in the WAS samples. These estimated concentrations were compared to the measured TCOD concentration of the WAS to determine the active and endogenous COD fractions. The estimation of $Z_{b h}$ and $Z_{e}$ in the BR WAS is presented first. The $\mathrm{X}_{\text {STo concentration }}$ 
was then estimated by subtracting the sum of $\mathrm{Z}_{\mathrm{bh}}$ and $\mathrm{Z}_{\mathrm{e}}$ from the TCOD of the BR WAS. Estimates of the active and endogenous COD fractions in the pretreated BR WAS are subsequently presented. Lastly, the estimation of the active fractions in the $\mathrm{AD}$ and corresponding endogenous fractions during Phases 1 and 2 are presented.

The literature review presented in section 2.1.5.1 discussed two methods to estimate the active fraction of the BR WAS. However, in this study, the OUR response measured in batch respirometry bottles containing only BR WAS showed both decay and growth and hence the active fraction could not be determined by fitting the endogenous OUR equation 2.4. The results suggested that in these batch tests, the stored COD in the BR WAS was used to generate new biomass. Hence, the concentration of active heterotrophs in the BR WAS was determined by the offline respirometric test carried out on day 278 on sample bottles containing BR WAS and synthetic feed in high F/M ratios.

Figure 4.22a presents a typical respirogram obtained from this test and Figure 4.22b shows the exponential growth portion of the curve. Using the data from Figure $4.22 \mathrm{~b}, \ln (\mathrm{OUR})$ versus time was plotted in Figure 4.22c. The active heterotroph concentration in the sample bottles $\left(\mathrm{Z}_{\mathrm{bh} 0}\right)$ was determined by equation 2.3 using the slope and y-intercept from the plot of Figure $4.22 \mathrm{c}$ and assuming typical values for $Y_{h}$ and $b_{h}$. These three graphs were plotted for each sample bottle and the active heterotroph concentration in each bottle was calculated. For each bottle, the concentration obtained using equation 2.3 was then multiplied by the dilution factor in the bottle to estimate the concentration of active heterotrophs in the BR WAS $\left(\mathrm{Z}_{\mathrm{bh}, \mathrm{BR}}\right)$. 

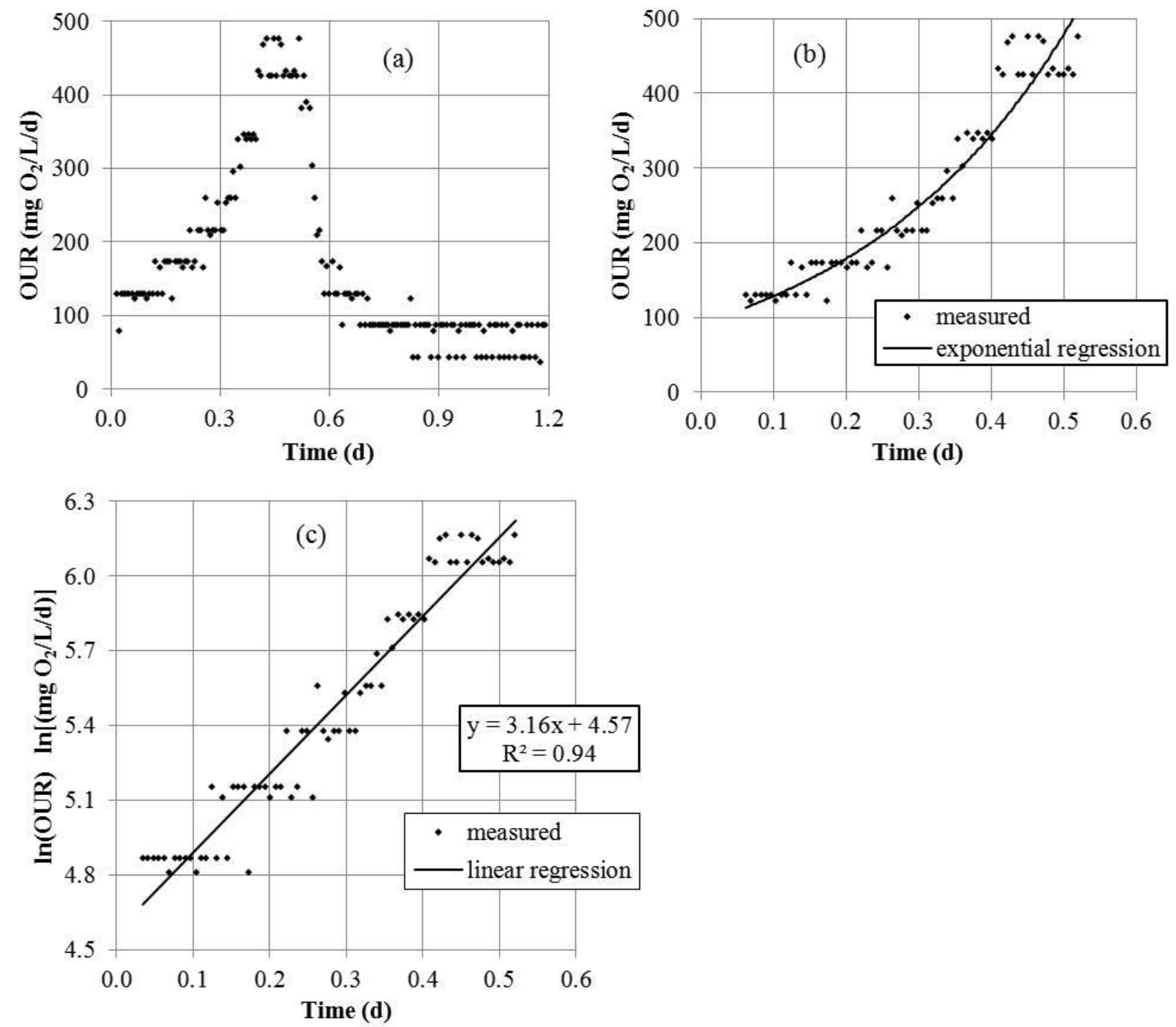

Figure 4.22 Typical Respirometry Data Used to Estimate $\mathbf{Z}_{\mathrm{bh}, 0}$ (a) Total Respirogram (b) Exponentially Increasing Portion of Respirogram (c) Log of Exponentially Increasing Portion

The average concentration of active heterotrophs in the BR WAS was estimated to be $2133 \pm 73 \mathrm{mg}$ $\mathrm{COD} / \mathrm{L}$. As reported in section 4.2.2, the average TCOD concentration in the BR WAS was $4170 \pm$ $324 \mathrm{mg} / \mathrm{L}$. Therefore the average active fraction of the BR WAS was $51 \pm 4 \%$. This was $17 \%$ lower than the active fraction reported by Ramdani et al. (2012) who operated an MBR with a 5.2 day SRT fed with sodium acetate as the sole carbon source. The active fraction was expected to be lower in the BR WAS because a portion of the biodegradable synthetic substrate was believed to be stored inside the cells in the BR WAS whereas the WAS employed by Ramdani et al. (2012) did not contain stored COD. 
The concentration of endogenous decay products was determined by the endogenous respiration approach:

$$
Z_{e, B R}=f b_{h} Z_{b h, B R}\left(S R T_{B R}\right) \quad(\text { Parker, 2010) }
$$

Where $b_{h}=0.28 \mathrm{~d}^{-1}$ at $25^{\circ} \mathrm{C}$ and $\mathrm{f}=0.2$. The dataset of concentrations estimated for the active heterotrophs as per equation 2.3 was substituted into equation 4.14 to generate a dataset of concentrations for the endogenous decay products. The average COD concentration of endogenous products in the BR WAS was $512 \pm 17 \mathrm{mg} / \mathrm{L}$ and the average endogenous COD fraction was $12 \pm$ $1 \%$. This was $20 \%$ lower than the endogenous fraction reported by Ramdani et al. (2012) who operated a similar system. The lower fraction was expected because the BR WAS contained stored COD whereas the WAS used by these authors did not. The presence of stored COD would result in less grown biomass and hence less decay of biomass to produce $\mathrm{Z}_{\mathrm{e}}$. The measured ffCOD in the BR WAS was $53 \pm 12 \mathrm{mg}$ COD/L which was only $1 \%$ of the TCOD. Thus the soluble non-biodegradable COD fraction was considered negligible. The sum of the active and endogenous COD fractions was $63 \pm 4 \%$ hence the remaining $37 \pm 4 \%$ was assumed to be stored COD. This estimate of $\mathrm{f}_{\text {STO }}$ was comparable to the estimate derived from the measured pON/PCOD value $\left(f_{\text {STO }}=25 \pm 9 \%\right)$ and the estimate derived from the measured COD/VSS ratio $\left(\mathrm{f}_{\mathrm{STO}}=54 \pm 28 \%\right)$.

Based on the literature review of HPTH pretreatment studies, it was expected that pretreatment would substantially if not completely inactivate the heterotroph population in the WAS. In this study, the active fraction was determined from the OUR response in the sample bottles containing only pretreated BR WAS. The $\mathrm{Z}_{\mathrm{bh} 0}$ concentration in the sample bottles containing pretreated BR WAS was determined by plotting $\ln (\mathrm{OUR})$ versus time for the exponentially increasing portion of the measured OUR curve and fitting equation 2.3 with typical values for $Y_{h}$ and $b_{h}$. The $Z_{b h 0}$ concentration was then multiplied by the dilution factor in the bottle to determine the concentration of active heterotrophs in the pretreated BR WAS $\left(\mathrm{Z}_{\mathrm{bh}, \mathrm{PT}}\right)$. The active fraction in the pretreated BR WAS was consistently found to be less than 5\%. For the purpose of fractionating the COD, it was therefore assumed that pretreatment fully inactivated the biomass.

The constant measured VSS concentration in the AD during Phases 1 and 2 and the results of the online and offline respirometric tests showed that the biodegradable COD fraction (bCOD) of the WAS was unchanged by pretreatment with an acclimatized biomass. It was therefore assumed that none of the endogenous residue was converted to biodegradable COD by pretreatment. Since the TCOD of the WAS was unchanged by pretreatment, the endogenous fraction of the pretreated WAS was assumed to be equivalent to that of the BR WAS, i.e. $12 \pm 1 \%$. 
The concentration of active heterotrophs in the bottles containing AD WAS was determined by fitting equation 2.4 by nonlinear regression to the measured OUR in these bottles. This concentration was then multiplied by the dilution factor in the bottle to estimate the concentration of active heterotrophs in the AD WAS $\left(\mathrm{Z}_{\mathrm{bh}, \mathrm{AD}}\right)$. It is believed that this approach was valid because the measured OUR response was typical of that associated with endogenous decay. The concentration of active heterotrophs in the steady-state AD was found to be $1200 \pm 260 \mathrm{mg} \mathrm{COD} / \mathrm{L}$ in Phase 1 and $700 \pm 150$ $\mathrm{mg}$ COD/L in Phase 2. The average TCOD of the AD WAS throughout the project was $2174 \pm 188$ $\mathrm{mg} \mathrm{COD} / \mathrm{L}$ hence the average active fraction was $55 \pm 13 \%$ in Phase 1 and $32 \pm 7 \%$ in Phase 2 . A ttest at the $95 \%$ confidence level showed no significant difference between the average active fraction in the BR WAS and that in the Phase 1 AD WAS. It was expected that the active fraction of the AD WAS would be less than that of the BR WAS in Phase 1 without pretreatment. However, unlike the BR WAS, the AD WAS did not contain stored COD as: the measured COD/VSS ratio of the AD WAS was typical of that for active biomass and decay products; and the OUR response in the AD WAS showed only an endogenous decay response. Therefore, it is believed that the biomass in the AD oxidized the storage products present in the BR WAS for energy production and cell synthesis. The active fraction in the AD WAS was therefore comprised of active biomass from the BR WAS that was undergoing endogenous decay in the digester and new biomass that grew in the digester on the storage products contained in the BR WAS. This would explain the higher than expected active fraction of the AD WAS in Phase 1.

The endogenous residue in the $\mathrm{AD}$ was assumed to consist of the sum of that originating from the $\mathrm{BR}$ WAS and that generated in the AD and was estimated using equation 4.15:

$$
Z_{e, A D}=f b_{h} Z_{b h, A D} S R T_{A D}+Z_{e, B R}
$$

Where $b_{h}=0.28 \mathrm{~d}^{-1}$ at $25^{\circ} \mathrm{C}$ and $\mathrm{f}=0.2$. The concentrations estimated for the active heterotrophs in the AD in Phases 1 and 2 were substituted into equation 4.15 to generate corresponding values for the concentrations of the endogenous decay products. The average $Z_{\mathrm{e}, \mathrm{AD}}$ value in Phase 1 was estimated to be $1090 \pm 350 \mathrm{mg} \mathrm{COD} / \mathrm{L}$ and hence the endogenous COD fraction was estimated to be $50 \pm 17 \%$. In Phase 2, the $Z_{e, A D}$ value was estimated to be $850 \pm 270 \mathrm{mg} \mathrm{COD} / \mathrm{L}$ and the endogenous COD fraction was estimated to be $39 \pm 13 \%$. The active and endogenous COD fractions in the BR, pretreated and AD WAS as well as the stored COD fraction in the BR WAS will be summarized in section 4.3.2.4. 


\subsubsection{COD Fractionation of the WAS}

Figure 4.23 summarizes the COD fractions in each sludge stream that could be estimated from the compiled results of the conventional analyses and offline respirometric tests. Only significant COD fractions are shown. The sludge streams included the BR WAS, pretreated BR WAS, AD WAS treating raw BR WAS (Phase 1) and AD WAS treating pretreated BR WAS (Phase 2). The COD fractions presented in Figure 4.23 were also estimated by simulating the entire system with calibrated activated sludge models and this will be discussed further in chapter 5 .

BR WAS
\begin{tabular}{|c|c|c|c|c|}
\hline $37 \pm 4 \%$ & $\mathrm{X}_{\text {STO }}$ & & $\mathrm{S}_{\mathrm{bsc}}$ & $?$ \\
\hline $51 \pm 4 \%$ & $\mathrm{Z}_{\mathrm{bh}}$ & & $\mathrm{X}_{\mathrm{sp}}$ & $?$ \\
\hline $12 \pm 1 \%$ & $\mathrm{Z}_{\mathrm{e}}$ & $\mathrm{Z}_{\mathrm{e}}$ & $12 \pm 1 \%$ \\
\hline
\end{tabular}
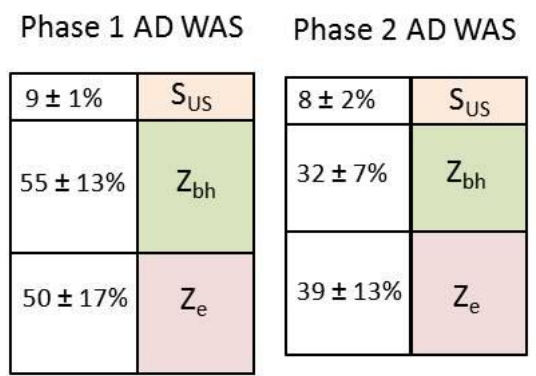

Figure 4.23 COD Fractionation of Sludge Streams

The BR WAS was comprised of $Z_{\mathrm{bh}}, Z_{\mathrm{e}}$ and $\mathrm{X}_{\mathrm{STO}}$. As described in section 4.3.2.3, the COD fractions attributed to $\mathrm{Z}_{\mathrm{bh}}$ and $\mathrm{Z}_{\mathrm{e}}$ were estimated to be $51 \pm 4 \%$ and $12 \pm 1 \%$, respectively, based on analyses of the offline respirometric data. The stored COD fraction, $\mathrm{f}_{\mathrm{STO}}$, was estimated in three different ways: using the COD/VSS ratios $\left(f_{\text {STO }}=54 \pm 28 \%\right)$; using the pON/PCOD ratios $\left(f_{\text {STO }}=25 \pm 9 \%\right.$ ); and using the COD not attributed to active and endogenous fractions determined by offline respirometry $\left(\mathrm{f}_{\mathrm{STO}}=37 \pm 4 \%\right)$. As discussed in section 4.2.3, the first approach was probably less accurate than the second because only one value was assumed in the first whereas two values were assumed in the second. A t-test at the $95 \%$ confidence level showed that the $\mathrm{f}_{\text {Sто }}$ estimates from the second and third approaches differed significantly. However, this difference was only $12 \%$ which was considered relatively small. Hence, for the purpose of COD closure, the COD fraction attributed to $\mathrm{X}_{\mathrm{STO}}$ was assumed to be $37 \pm 4 \%$ in Figure 4.23. The BR WAS contained an average measured ffCOD concentration of $53 \pm 12 \mathrm{mg} / \mathrm{L}$ which was assumed to be $S_{\text {us }}$ from SMPs. This fraction comprised only $1.3 \pm 0.3 \%$ of the TCOD of the BR WAS hence it was considered negligible in the overall COD fractionation presented in Figure 4.23.

The pretreated BR WAS was assumed to be comprised of $S_{\mathrm{bsc}}, \mathrm{X}_{\mathrm{sp}}$ and $\mathrm{Z}_{\mathrm{e}}$. It was shown that pretreatment did not change the TCOD of the BR WAS nor did it change the biodegradable fraction. Therefore the endogenous fraction in the pretreated BR WAS was assumed to be equivalent to that in 
the BR WAS, i.e. $12 \pm 1 \%$. It was assumed that $\mathrm{X}_{\mathrm{STO}}$ and $\mathrm{Z}_{\mathrm{bh}}$ in the BR WAS were converted to a mixture of $S_{\mathrm{bsc}}$ and $X_{\mathrm{sp}}$ in the pretreated BR WAS. As shown in section 4.2.3, pretreatment increased the average SCOD concentration in the BR WAS from $1 \%$ to $57 \%$. Since it was shown in section 4.2.4 that pretreatment did not generate $S_{\text {us }}$, the SCOD generated by pretreatment was expected to be $\mathrm{S}_{\mathrm{bsc}}$. Because this $\mathrm{S}_{\mathrm{bsc}}$ generated by pretreatment was significantly higher than the $\mathrm{X}_{\mathrm{STO}}$ estimated to be present in the BR WAS, it was assumed that $X_{\text {STо }}$ had been fully converted to $S_{\text {bsc }}$. The storage products were assumed to be released when the cells were inactivated by pretreatment. The balance

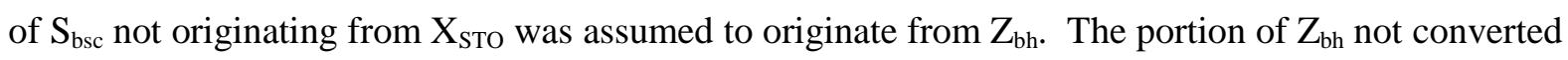
to $S_{\mathrm{bsc}}$ was assumed to be converted to $\mathrm{X}_{\mathrm{sp}}$. The $\mathrm{S}_{\mathrm{bsc}}$ and $\mathrm{X}_{\mathrm{sp}}$ concentrations in the pretreated BR WAS were estimated by fitting OUR responses predicted by activated sludge models to the measured offline respirometric data. This work will be presented in chapter 5 .

The AD WAS was comprised of $S_{\mathrm{us}}, \mathrm{Z}_{\mathrm{bh}}$ and $\mathrm{Z}_{\mathrm{e}}$ in Phases 1 and 2. The $\mathrm{S}_{\mathrm{us}}$ was believed to be generated by the decay of cells that stored COD. As shown in section 4.2.4, the average measured ffCOD concentration in the AD WAS was $190 \pm 11 \mathrm{mg}$ COD/L in Phase 1 and $177 \pm 31 \mathrm{mg}$ COD/L in Phase 2. Thus the average $S_{\text {us }}$ fraction was $9 \pm 1 \%$ in Phase 1 and $8 \pm 2 \%$ in Phase 2. Both of these fractions are substantial and were therefore included in the overall fractionation of the AD WAS presented in Figure 4.23. The sum of the average calculated $S_{u s}, Z_{b h}$ and $Z_{e}$ concentrations in the Phase $1 \mathrm{AD}$ WAS was $2480 \pm 436 \mathrm{mg} / \mathrm{L}$ and a t-test at the $95 \%$ confidence level showed no significant difference between this sum and the average measured TCOD of the Phase 1 AD WAS. This mass balance closure suggested that the active and endogenous fractions were well estimated. However, the sum of the average calculated $S_{u s}, Z_{b h}$ and $Z_{e}$ concentrations in the Phase 2 AD WAS was $1727 \pm 310 \mathrm{mg} / \mathrm{L}$ which was $447 \mathrm{mg} / \mathrm{L}$ less than the average measured TCOD of the Phase $2 \mathrm{AD}$ WAS. A t-test at the $95 \%$ confidence level showed that this difference was significant. This lack of mass balance closure may have been due to inaccuracies in the respirometric measurements, error in the nonlinear regression fit of equation 2.4 to the respirometric data or inaccuracies in the parameter estimates for $b_{h}, f$ and $Y_{h}$. As will be discussed in sections 5.2 and 5.3, the COD fractions in the various sludge streams were also estimated using calibrated activated sludge models thus the COD fractionation of the Phase 2 AD WAS will be revisited. 


\section{Development of the Pretreatment Model}

The results of the conventional analyses and respirometric tests were previously used to fractionate the COD of the various sludge streams in order to identify the COD transformations caused by pretreatment. It was concluded that pretreatment did not alter the aerobically biodegradable fraction of the BR WAS. However, the results of the batch respirometric tests indicated that pretreatment increased the rate of aerobic degradation. The experimental results presented in section 4 could not be directly used to quantify the concentrations of $S_{\mathrm{bsc}}$ and $\mathrm{X}_{\mathrm{sp}}$ in the pretreated BR WAS. In this chapter these concentrations will be estimated using activated sludge models. Furthermore, modeling was employed to verify the COD fractions in the various sludge streams that were estimated from the results presented in section 4 . An overview of the modeling procedure is discussed first.

The Phase 1 system was simulated using two different models, the BioWin 3.1® Integrated Sludge Model and the ASM3 Model. The models were initially calibrated by systematically adjusting key kinetic and stoichiometric parameters within their typical ranges such that the predicted concentrations of particulate COD species in the BR and AD WAS were statistically equivalent to the respective average measured concentrations. The accuracy of the parameter estimates was then improved by minimizing the sum of squared differences between the predicted and measured OUR responses from offline respirometry on BR WAS. The calibrated model that best fit the measured data was then employed to simulate the Phase 2 system. The concentrations of $S_{\mathrm{bsc}}$ and $\mathrm{X}_{\mathrm{sp}}$ in the pretreated BR WAS were adjusted such that the predicted concentrations of PCOD and SCOD in the pretreated BR WAS and PCOD in the Phase 2 AD WAS were statistically equivalent to the respective average measured concentrations. The accuracy of the estimated $S_{\mathrm{bsc}}$ and $\mathrm{X}_{\mathrm{sp}}$ concentrations was then improved using a least squares regression between the predicted and measured OUR responses from offline respirometry on inoculated pretreated BR WAS. Once the $S_{b s c}$ and $X_{s p}$ concentrations had been estimated, the COD fractionation of the pretreated BR WAS was complete. A COD-based stoichiometric pretreatment model was then developed for the dose of HPTH pretreatment employed in this study. This model was evaluated and its limitations were identified. 


\subsection{Modeling Approach}

This section describes the approach taken to simulate the startup and operation of the BR-AD system using two different activated sludge models. An overview of the operation of the reactors throughout the project is first discussed. As described in section 4.1, the BR was operated as an SBR with an SRT of $5 \mathrm{~d}$. The BR was initially seeded with activated sludge and then fed daily with a synthetic wastewater. The concentrations of alkalinity and inorganic nutrients in the synthetic wastewater were specified according to the theoretical composition. The TCOD and COD fractionation of the synthetic wastewater were estimated based on COD measurements and required some assumptions. On day 20, the AD was started up by being filled with $2 \mathrm{~L}$ of BR WAS per day until it contained $6 \mathrm{~L}$. The AD continued to be fed daily with BR WAS until the end of the project. The AD was initially operated as an SBR with an HRT of $3 \mathrm{~d}$ and an SRT of $10 \mathrm{~d}$. On day 77, the operation of the AD was simplified so that the HRT and SRT were both $10 \mathrm{~d}$. The concentration of the synthetic wastewater fed to the BR was halved on day 179 and this feed concentration was maintained until the end of the project on day 283. The focus period of the project consisted of two phases: Phase 1 from day 179 to 203 when the AD was fed with raw BR WAS; and Phase 2 from day 203 to 283 when the AD was fed with pretreated BR WAS.

The startup and operation of the BR-AD system was simulated using the BioWin 3.1® Integrated Model in the BioWin ${ }^{\circledR}$ platform. The system was simulated exactly as it was operated from day 0 to 283. The change in the operation of the AD implemented on day 77 and the change in the concentration of the synthetic wastewater implemented on 179 were included in the simulation and it was predicted that a new steady state condition would be reached following each change. The results of the conventional analyses confirmed this prediction. As discussed in section 4.2.2, the concentrations of COD and SS species measured by conventional analyses remained relatively stable from day 197 until day 283, indicating that the BR was at steady state during this period. The concentrations of TCOD, SCOD, ffCOD and VSS measured in the AD during this period also remained relatively constant, showing that the AD was at steady state as well.

It was hypothesized that the steady-state conditions of the Phase 1 BR-AD system only depended on the operating conditions and synthetic wastewater recipe employed during this phase. This assumption was tested using a separate, simplified simulation in which the BR was seeded with a typical activated sludge, fed synthetic wastewater with the composition used from day 179 onwards 
and then allowed to reach steady-state. The $\mathrm{AD}$ was then started up and operated as it had been during Phase 1, until it stabilized. The steady-state compositions of the sludge streams in this simplified simulation were found to be equivalent to the compositions of the respective sludge streams in the Phase 1 steady-state system achieved by modeling the actual operation from day 0. The simplified approach was therefore deemed adequate to model the Phase 1 BR-AD system.

In addition to the BioWin 3.1® Integrated Model, the Phase 1 BR-AD system was also modeled with the ASM3 Model in the BioWin® platform. Although the BioWin 3.1® Integrated Model is more complex and more widely used than the ASM3 Model, it does not include processes for the storage of COD by ordinary heterotrophic biomass that does not accumulate phosphorus $\left(\mathrm{Z}_{\mathrm{bh}}\right)$. The results presented in section 4 showed that the BR WAS contained stored COD in addition to active heterotrophs and decay products. In the ASM3 Model, all readily biodegradable substrate is first stored inside cells before it is used for growth and endogenous respiration. It was therefore hypothesized that the ASM3 Model would be well suited for this project.

The configuration of the Phase 1 BR-AD system used in the BioWin ${ }^{\circledR}$ platform is shown in Figure 5.1. The overall system was simulated at $20^{\circ} \mathrm{C}$ as this was the average temperature at which the $\mathrm{BR}$ and $\mathrm{AD}$ were operated. In the simulations, the concentrations of the COD, SS and nitrogen species were monitored in each sludge stream so that the predicted steady-state concentrations could be compared with the average measured concentrations. In addition, the OUR in the simulated AD was monitored so that it could be compared to the online respirometric results.

In order to simulate the batch respirometric tests, a variable volume reactor labeled "OUR Test" was included in the configuration in Figure 5.1. This reactor was simulated at $25^{\circ} \mathrm{C}$ because the offline respirometric tests were operated at this temperature. Once the BR-AD system was at steady-state, $150 \mathrm{~mL}$ of WAS from the BR was fed to the OUR Test reactor and the simulation was continued for a number of days. The DO concentration in the simulated batch reactor was held constant at $2 \mathrm{mg} / \mathrm{L}$ and the OUR in this reactor was monitored over time. Offline respirometry simulations were also carried out using $50 \mathrm{~mL}$ AD WAS only and a mixture of $150 \mathrm{~mL}$ of BR WAS and $50 \mathrm{~mL}$ of $\mathrm{AD}$ WAS. 


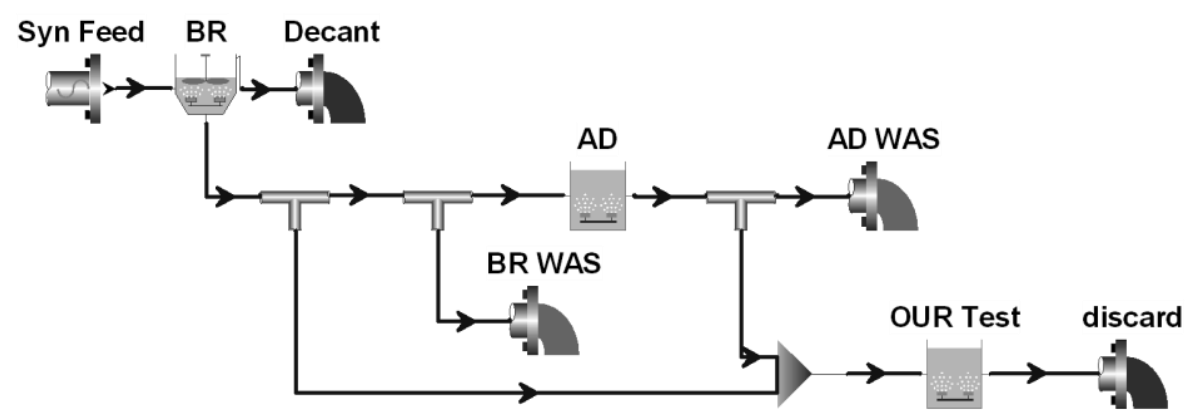

Figure 5.1 Configuration of Phase 1 BR-AD System in BioWin® ${ }^{\circledR}$ 3.1 Platform

It was shown in sections 4.2.2 and 4.2.4 that the WAS from the BR and AD contained concentrations of ffCOD and this was assumed to be $S_{\text {us }}$ from SMPs generated in the reactors. A significantly higher average concentration of $S_{\text {us }}$ was measured in AD WAS than in the BR WAS which suggested that the SMPs were generated by endogenous decay rather than by growth. Neither the BioWin 3.1® Integrated Model nor the ASM3 Model predicts the channeling of COD into SMPs for processes involving $\mathrm{Z}_{\mathrm{bh}}$. Therefore, the simulated synthetic wastewater was assigned a $\mathrm{S}_{\mathrm{us}}$ concentration equivalent to the average measured ffCOD concentration in the BR WAS. No provision was made to simulate the additional $S_{\text {us }}$ shown to be generated in the AD hence it was expected that both models would underestimate the ffCOD and SCOD concentrations in the AD.

During the focus period of the project, the nitrate concentrations measured in the BR and AD were consistently below $1 \mathrm{mg} \mathrm{N} / \mathrm{L}$, indicating that nitrification was negligible in both reactors. In order to simulate this response, the value of the maximum specific growth rate of the ammonia oxidizing biomass $\left(\mu_{\mathrm{a}}\right)$ in both the BioWin 3.1® Integrated Model and ASM3 Model was set to zero. Besides this parameter, the kinetic and stoichiometric parameters employed in each model differed considerably and will therefore be discussed separately in the next two sections. An overview of the capabilities of each model will also be presented.

\subsubsection{BioWin 3.1 ${ }^{\circledR}$ Integrated Model}

The BioWin 3.1® Integrated Model is a biochemical mass balance model that characterizes organic wastewater components in terms of COD. The model describes biological processes, chemical precipitation reactions and gas-liquid mass transfers using fifty state variables and sixty process expressions. Because it incorporates many different process units, the model is well suited for 
simulating entire WWTPs. This work focused on the processes within the overall model that address the behavior of heterotrophic biomass in aerobic systems and the associated consumption of substrates and generation of byproducts. The selection of the appropriate parameter values for these processes was an important element of the model implementation.

The values of $f$ and $b_{h}$ for heterotrophs that were employed in chapters 3 and 4 were 0.2 and $0.24 \mathrm{~d}^{-1}$, respectively, at $20^{\circ} \mathrm{C}$ (Henze et al., 2008). A value of 0.6 was assumed for $\mathrm{Y}_{\mathrm{h}}$ since this was the value reported by Ramdani et al. (2012) for acetate-fed systems. These values are based on the endogenous respiration approach to describe the kinetics of cell maintenance, endogenous metabolism, decay, lysis and death. However, the BioWin 3.1® Integrated Model employs the death-regeneration approach. The corresponding values of $f$ and $b_{h}$ using this approach were 0.09 and $0.53 \mathrm{~d}^{-1}$ at $20^{\circ} \mathrm{C}$, respectively. The value of $b_{h}$ was calculated to be $0.61 \mathrm{~d}^{-1}$ at $25^{\circ} \mathrm{C}$ using equation 4.12 . The $\mathrm{f}$ value is considered to be independent of temperature.

Besides $\mu_{\mathrm{a}}, \mathrm{b}_{\mathrm{h}}, \mathrm{Y}_{\mathrm{h}}$ and $\mathrm{f}$, the default values in the BioWin ${ }^{\circledR}$ 3.1 Integrated Model were used for the other kinetic and stoichiometric parameters. As mentioned, the model was used to simulate the Phase 1 BR-AD system. The initial simulation employed $Y_{h}$, $f$ and $b_{h}$ values of $0.6,0.09$ and $0.53 \mathrm{~d}^{-1}$ at $20^{\circ} \mathrm{C}$, respectively, and then the model was calibrated by systematically adjusting $\mathrm{Y}_{\mathrm{h}}, \mathrm{f}$ and $\mathrm{b}_{\mathrm{h}}$ within their respective typical ranges. This will be described in section 5.2.

\subsubsection{ASM3 Model}

The ASM3 Model simulates oxygen consumption, sludge production, nitrification and denitrification in activated sludge systems treating wastewater of primarily domestic origin. Compared to the BioWin 3.1® Integrated Model, the ASM3 Model is much simpler as it has only thirteen state variables and twelve reactions. The ASM3 Model is designed to be the core of many different models. Although it does not include modules for biological phosphorus removal, chemical precipitation, growth of filamentous organisms or $\mathrm{pH}$ calculations, they can easily be connected as add-on modules (Henze et al., 2000).

The matrix representation of the ASM3 Model in the BioWin platform is presented in Table 5.1. This matrix shows only the processes that were believed to be involved in the Phase 1 BR-AD system. Therefore, anoxic processes and the growth and endogenous respiration of autotrophs were omitted 
because these processes were determined to be negligible. The BR and AD were always operated aerobically and the measured nitrate levels in the various sludge streams were consistently below 1 $\mathrm{mg} \mathrm{N} / \mathrm{L}$. Both the stoichiometric matrix and kinetic rate expressions shown in Table 5.1 were adapted to the BioWin ${ }^{\circledR}$ platform from the ASM3 Model proposed by Henze et al. (2000). The matrix employs symbols specific to the BioWin ${ }^{\circledR}$ platform for compounds and kinetic and stoichiometric parameters. The ASM3 Model does not include a variable for acetate $\left(\mathrm{S}_{\mathrm{bsa}}\right)$ thus all rbCOD was represented as $S_{\text {bsc }}$. Furthermore, the ASM3 Model does not include a variable for slowly biodegradable colloidal COD $\left(\mathrm{X}_{\mathrm{sc}}\right)$ thus all slowly biodegradable COD was represented as $\mathrm{X}_{\mathrm{sp}}$. The ASM3 Model does not differentiate endogenous residue from other non-biodegradable particulate COD and therefore represented both as $\mathrm{X}_{\mathrm{i}}$. Stored COD in the ASM3 Model is represented by the symbol $S_{\text {phb }}$ in the BioWin ${ }^{\circledR}$ platform. Therefore, $S_{\text {phb }}$ will be used to denote stored COD in this chapter. The ASM3 Model matrix presented by Henze et al. (2000) included independent variables for alkalinity and suspended solids however each of these variables is a combined variable in the BioWin ${ }^{\circledR}$ platform. Therefore, these variables were calculated according to the BioWin method when the BioWin ${ }^{\circledR}$ platform was used to run the ASM3 Model. 
Table 5.1 ASM3 Model in BioWin ${ }^{\circledR}$ Platform Showing Processes in Phase 1 BR-AD System

\begin{tabular}{|c|c|c|c|c|c|c|c|c|}
\hline \multirow{2}{*}{ Process } & DO & $\mathbf{S}_{\mathrm{bsc}}$ & $\mathrm{NH}_{3}-\mathrm{N}$ & $\mathbf{X}_{\mathbf{i}}$ & $\mathbf{X}_{\mathrm{sp}}$ & $\mathbf{Z}_{\mathrm{bh}}$ & $\mathbf{S}_{\text {phb }}$ & \multirow[b]{2}{*}{ Kinetic Rate Expressions } \\
\hline & $\begin{array}{l}\mathrm{mg} \\
\mathrm{O}_{2} / \mathrm{L}\end{array}$ & $\begin{array}{l}\mathrm{mg} \\
\mathrm{COD} / \\
\mathrm{L}\end{array}$ & $\mathrm{mg} \mathrm{N} / \mathrm{L}$ & \multicolumn{4}{|c|}{ mg COD/L } & \\
\hline \multirow{2}{*}{ Hydrolysis } & & \multirow{2}{*}{1} & \multirow{2}{*}{$\begin{array}{l}-i_{N S S} \\
+i_{N X S}\end{array}$} & & \multirow{2}{*}{-1} & & & $k_{H} \times \frac{X_{s p}}{0.0001+Z_{b h}}$ \\
\hline & & & & & & & & $\begin{aligned} & K_{X}+\frac{X_{s p}}{0.0001+Z_{b h}} \\
& \times Z_{b h}\end{aligned}$ \\
\hline $\begin{array}{l}\text { Aerobic storage } \\
\text { of } S_{b s c} \text { by } Z_{b h}\end{array}$ & $\begin{array}{l}Y_{S T O, 02} \\
-1\end{array}$ & -1 & $i_{N S S}$ & & & & $Y_{S T O, O 2}$ & $\begin{aligned} k_{S T O} \times \frac{D O}{K_{O}+D O} & \times \frac{S_{b s c}}{K_{S}+S_{b s c}} \\
& \times Z_{b h}\end{aligned}$ \\
\hline \multirow[t]{2}{*}{$\begin{array}{l}\text { Aerobic growth of } \\
Z_{\mathrm{bh}} \text { on } S_{\mathrm{phb}}\end{array}$} & \multirow[t]{2}{*}{$\begin{array}{l}1 \\
-\frac{1}{Y_{H, O 2}}\end{array}$} & & \multirow[t]{2}{*}{$-i_{N B M}$} & & & \multirow[t]{2}{*}{1} & $-\frac{1}{Y_{H, O 2}}$ & \multirow{2}{*}{$\begin{array}{l}\mu_{H} \times \frac{D O}{K_{O}+D O} \\
\times \frac{N H_{3} N}{K_{N H}+N H_{3} N} \\
\times \frac{S_{p h b}}{0.0001+Z_{b h}} \\
\times \frac{1}{k_{S T O}+\frac{S_{p h b}}{0.0001+Z_{b h}}} \\
\times Z_{b h}\end{array}$} \\
\hline & & & & & & & & \\
\hline $\begin{array}{l}\text { Aerobic } \\
\text { endogenous } \\
\text { respiration of } Z_{b h}\end{array}$ & $f_{i}-1$ & & $\begin{array}{l}i_{N B M} \\
-\left(f_{i}\right. \\
\left.\times i_{N X I}\right) \\
\end{array}$ & $f_{i}$ & & -1 & & $b_{H, O 2} \times \frac{D O}{K_{O}+D O} \times Z_{b h}$ \\
\hline $\begin{array}{l}\text { Aerobic } \\
\text { respiration of } S_{\mathrm{phb}}\end{array}$ & -1 & & & & & & -1 & $b_{H, O 2} \times \frac{D O}{K_{O}+D O} \times S_{p h b}$ \\
\hline
\end{tabular}

The values of the kinetic and stoichiometric parameters that were described in Table 5.1 are presented in Table 5.2. These values were determined by Koch et al. (2000) for the calibrated ASM3 Model at $20^{\circ} \mathrm{C}$. The values of temperature-dependent kinetic parameters were calculated at $25^{\circ} \mathrm{C}$ using the corresponding coefficient $\theta_{\mathrm{T}}$ reported by Koch et al. (2000) and the ASM3 temperature equation presented by Henze et al. (2000). When the ASM3 Model was run in the BioWin ${ }^{\circledR}$ platform, the COD/VSS ratio of storage products was specified as $1.1 \mathrm{gCOD} / \mathrm{gVSS}$ because this is the typical value for glycogen which was believed to be stored inside the cells. The particulate inert COD/VSS ratio was specified as $1.42 \mathrm{gCOD} / \mathrm{gVSS}$ because the inert fraction was expected to be solely comprised of endogenous residue. 
Table 5.2 Kinetic and Stoichiometric Parameters in ASM3 Model for Phase 1 BR-AD System

\begin{tabular}{|c|c|c|c|c|}
\hline Kinetic Parameter & Symbol & Unit & $\begin{array}{l}\text { Value } \\
\left(20^{\circ} \mathrm{C}\right)\end{array}$ & $\begin{array}{l}\text { Value } \\
\left(25^{\circ} \mathrm{C}\right)\end{array}$ \\
\hline Hydrolysis rate constant & $k_{H}$ & $d^{-1}$ & 9 & 11 \\
\hline Hydrolysis saturation constant & $K_{X}$ & $\frac{g X_{s p}}{g Z_{b h}}$ & \multicolumn{2}{|c|}{1} \\
\hline Aerobic storage rate constant & $k_{S T O}$ & $\frac{g S_{b s c}}{g Z_{b h} d}$ & 12 & 17 \\
\hline Inhibition constant for oxygen (DO) & $K_{O}$ & $\frac{g \mathrm{O}_{2}}{m^{3}}$ & \multicolumn{2}{|c|}{0.2} \\
\hline Saturation constant for substrate $\left(\mathrm{S}_{\mathrm{bsc}}\right)$ & $K_{S}$ & $\frac{g C O D}{m^{3}}$ & \multicolumn{2}{|c|}{10} \\
\hline Saturation constant for storage & $K_{S T O}$ & $\frac{g S_{p h b}}{g Z_{b h}}$ & \multicolumn{2}{|c|}{0.1} \\
\hline Heterotrophic maximum aerobic growth rate & $\mu_{H}$ & $d^{-1}$ & 3 & 4.3 \\
\hline Saturation constant for ammonia $\left(\mathrm{NH}_{3}-\mathrm{N}\right)$ & $K_{N H}$ & $\frac{g N}{m^{3}}$ & \multicolumn{2}{|c|}{0.1} \\
\hline Aerobic endogenous respiration rate of $\mathrm{Z}_{\mathrm{bh}}$ & $b_{H, 02}$ & $d^{-1}$ & 0.3 & 0.4 \\
\hline Production of $\mathrm{X}_{\mathrm{i}}$ in endogenous biomass respiration & $f_{i}$ & $\frac{g X_{i}}{g Z_{b h}}$ & \multicolumn{2}{|c|}{0.2} \\
\hline Aerobic yield of stored products per $S_{b s c}$ & $Y_{S T O, 02}$ & $\frac{g S_{p h b}}{g S_{b s c}}$ & \multicolumn{2}{|c|}{0.80} \\
\hline Aerobic yield of heterotrophic biomass growth on $S_{\mathrm{phb}}$ & $Y_{H, O 2}$ & $\frac{g Z_{b h}}{g S_{p h b}}$ & \multicolumn{2}{|c|}{0.80} \\
\hline Nitrogen content of $S_{\mathrm{bsc}}$ & $i_{N S S}$ & $\frac{g N}{g C O D}$ & \multicolumn{2}{|c|}{0.03} \\
\hline Nitrogen content of $X_{i}$ & $i_{N X I}$ & $\frac{g N}{g C O D}$ & \multicolumn{2}{|c|}{0.04} \\
\hline Nitrogen content of $\mathrm{X}_{\mathrm{sp}}$ & $i_{N X S}$ & $\frac{g N}{g C O D}$ & \multicolumn{2}{|c|}{0.03} \\
\hline Nitrogen content of $Z_{b h}$ & $i_{N B M}$ & $\frac{g N}{g C O D}$ & \multicolumn{2}{|c|}{0.07} \\
\hline
\end{tabular}

The ASM3 Model with the values presented in Table 5.2 was used in the BioWin ${ }^{\circledR}$ platform to simulate the Phase $1 \mathrm{BR}-\mathrm{AD}$ system. The predicted responses were then matched to the measured data by systematically adjusting $\mu_{\mathrm{H}}$ and $\mathrm{b}_{\mathrm{H}, \mathrm{O} 2}$, as will be described in section 5.2. 


\subsection{Phase 1 System Modeling}

The BioWin 3.1® Integrated Model and the ASM3 Model were each used to simulate the Phase 1 BR-AD system. A trial and error approach was used to initially estimate the values of the key kinetic and stoichiometric parameters such that the predicted concentrations of particulate COD species in the $\mathrm{BR}$ and $\mathrm{AD}$ WAS were statistically equivalent to the corresponding average measured concentrations using t-tests at the $95 \%$ confidence level. Following this, a least squares regression between the predicted and measured OUR responses from offline respirometry on BR WAS was employed to improve the accuracy of the estimates of the parameter values.

Using a trial and error approach, the value of $Y_{h}$ was adjusted while $f$ and $b_{h}$ were held constant at their initially assumed values, i.e. 0.09 and $0.53 \mathrm{~d}^{-1}$, respectively. For each new value of $Y_{h}$, the system was simulated using the BioWin 3.1® Integrated Model. The values of $Y_{h}$ that yielded simulated particulate COD concentrations in the reactors that were statistically equivalent to respective measured concentrations were recorded. Using these $Y_{h}$ values, the offline respirometric test on BR WAS was then simulated. The $Y_{h}$ value that minimized the sum of squared differences between predicted and measured OUR responses was thus determined. This approach was repeated a second time by adjusting $f$ while keeping $b_{h}$ and $Y_{h}$ constant at $0.53 \mathrm{~d}^{-1}$ and 0.6 , respectively. This approach was repeated a third time by adjusting $b_{h}$ while keeping $f$ and $Y_{h}$ constant at 0.09 and 0.6, respectively. It was found that the model best fit the measured data when the values of $Y_{h}, f$ and $b_{h}$ were $0.67,0.09,0.53 \mathrm{~d}^{-1}$ at $20^{\circ} \mathrm{C}$, respectively. Thus the calibrated model employed the initially assumed values of $f$ and $b_{h}$ whereas the $Y_{h}$ value was changed from the initially assumed value of 0.6 that was developed from systems fed by acetate. This $\mathrm{Y}_{\mathrm{h}}$ of 0.67 was believed to be reasonable as it is the standard value reported by Henze et al. (2008) for ordinary heterotrophic organisms.

The same trial and error approach described above for the BioWin Model was used to calibrate the ASM3 Model except that the adjusted parameters were $\mathrm{b}_{\mathrm{H}, \mathrm{O} 2}$ and $\mu_{\mathrm{h}}$. The initially assumed values of $\mathrm{b}_{\mathrm{H}, \mathrm{O} 2}$ and $\mu_{\mathrm{h}}$ were $0.3 \mathrm{~d}^{-1}$ and $3 \mathrm{~d}^{-1}$, respectively. It was found that the best fit was achieved using $\mathrm{b}_{\mathrm{H}, \mathrm{O} 2}$ and $\mu_{\mathrm{h}}$ values of $0.3 \mathrm{~d}^{-1}$ and $0.56 \mathrm{~d}^{-1}$ at $20^{\circ} \mathrm{C}$, respectively. Thus the calibrated model employed the initially assumed value of $b_{\mathrm{H}, \mathrm{O} 2}$. However, the calibrated model employed a $\mu_{\mathrm{h}}$ value that was much lower than the initially assumed value of $3 \mathrm{~d}^{-1}$, i.e. the value reported by Koch et al. (2000). This indicated that the heterotrophic growth rate was lower in the current study. This difference may have 
been because the current study employed synthetic wastewater whereas Koch et al. (2000) used municipal wastewater.

Tables 5.3 and 5.4 present the measured concentrations of COD, SS and nitrogen species in the Phase 1 BR-AD system and the corresponding values predicted by the calibrated BioWin $3.1 ®$ Integrated Model and the calibrated ASM3 Model. Percent differences between corresponding simulated and measured values are shown and significant differences, as defined by t-test hypotheses at the $95 \%$ confidence level, are shaded grey.

Table 5.3 Measured and Simulated COD, SS and N Species in BR WAS

\begin{tabular}{|l||l|l||l|l||l|l|}
\hline \multirow{2}{*}{ Parameter } & \multicolumn{2}{|c||}{ Measured } & \multicolumn{2}{c||}{ BioWin } & \multicolumn{2}{|c|}{ ASM3 Model } \\
\cline { 2 - 7 } & Avg. & $\begin{array}{l}\text { Std. } \\
\text { dev. }\end{array}$ & Predicted & $\begin{array}{l}\text { Difference from } \\
\text { Measured (\%) }\end{array}$ & Predicted & $\begin{array}{l}\text { Difference from } \\
\text { Measured (\%) }\end{array}$ \\
\hline PCOD & 4127 & 334 & 4315 & 5 & 4218 & 2 \\
\hline SCOD & 52 & 14 & 53 & 2 & 53 & 2 \\
\hline ffCOD & 53 & 12 & 53 & 0 & 53 & 0 \\
\hline VSS & 3342 & 167 & 3039 & 9 & 3374 & 1 \\
\hline ISS & 489 & 103 & 320 & 35 & 358 & 27 \\
\hline $\mathrm{NO}_{3}-\mathrm{N}$ & 0.1 & 0.1 & 0 & 100 & 0 & 100 \\
\hline $\mathrm{NH}_{3}-\mathrm{N}$ & 73 & 11 & 71 & 3 & 70 & 4 \\
\hline
\end{tabular}

Concentrations in $\mathrm{mg} / \mathrm{L}$

Table 5.4 Measured and Simulated COD, SS and N Species in Phase 1 AD WAS

\begin{tabular}{|l||l|l||l|l||l|l|}
\hline \multirow{2}{*}{ Parameter } & \multicolumn{2}{|c||}{ Measured } & \multicolumn{2}{c||}{ BioWin } & \multicolumn{2}{|c|}{ ASM3 Model } \\
\cline { 2 - 7 } & Avg. & $\begin{array}{l}\text { Std. } \\
\text { dev. }\end{array}$ & Predicted & $\begin{array}{l}\text { Difference from } \\
\text { Measured (\%) }\end{array}$ & Predicted & $\begin{array}{l}\text { Difference from } \\
\text { Measured (\%) }\end{array}$ \\
\hline PCOD & 2119 & 218 & 2208 & 4 & 2142 & 1 \\
\hline SCOD & 309 & 30 & 53 & 83 & 53 & 83 \\
\hline ffCOD & 190 & 11 & 53 & 72 & 53 & 72 \\
\hline VSS & 1667 & 117 & 1577 & 5 & 1648 & 1 \\
\hline ISS & 980 & 85 & 745 & 24 & 732 & 25 \\
\hline $\mathrm{NO}_{3}$-N & 0.3 & 0.4 & 0 & 100 & 0 & 100 \\
\hline $\mathrm{NH}_{3}-\mathrm{N}$ & 112 & 9 & 108 & 4 & 113 & 1 \\
\hline
\end{tabular}

Concentrations in $\mathrm{mg} / \mathrm{L}$

As shown in Tables 5.3 and 5.4, both models were successfully calibrated in terms of predicting PCOD concentrations in the BR and AD WAS that were statistically equivalent to the respective average measured concentrations. As expected, the predicted SCOD and ffCOD concentrations in the 
BR and AD WAS were equivalent to the $S_{\text {us }}$ concentration in the synthetic feed, i.e. $53 \mathrm{mg} / \mathrm{L}$, because neither model addresses the generation of $S_{u s}$.

The predicted $\mathrm{NH}_{3}-\mathrm{N}$ concentrations in the WAS from the $\mathrm{BR}$ and $\mathrm{AD}$ processes were statistically equivalent to the respective average measured concentrations. This finding validated the model calibration as the $\mathrm{NH}_{3}-\mathrm{N}$ response was independent of the calibration. The predicted nitrate $\left(\mathrm{NO}_{3}-\mathrm{N}\right)$ concentrations significantly differed from measured levels. However, both predicted and measured concentrations were very close to zero and therefore considered negligible.

Neither model accurately predicted the ISS concentration in the BR or AD WAS. This was expected because the processes involving ISS were not well developed in either model. The predicted ISS response was not expected to significantly impact the behaviour of particulate COD throughout the system.

Compared to the measured VSS concentrations, the BioWin 3.1® Integrated Model predicted a 9\% lower concentration in the BR WAS and a 5\% lower concentration in the AD WAS. Both these differences were significant however the $5 \%$ difference was considered minimal. The $9 \%$ difference may have been due to the fact that the model employed the typical COD/VSS ratio of 1.42 whereas the average measured COD/VSS ratio of the BR WAS was $1.23 \pm 0.08$. Compared to the measured VSS concentrations, the ASM3 Model predicted a 1\% higher concentration in the BR WAS and a $1 \%$ lower concentration in the AD WAS however both these differences were insignificant. This finding validated the ASM3 Model calibration as the VSS response was independent of the calibration.

In addition to the PCOD concentrations, the calibration of the models was based on predicting $Z_{\mathrm{bh}}, \mathrm{Z}_{\mathrm{e}}$ and $S_{\mathrm{phb}}$ concentrations in the BR and AD WAS that were statistically equivalent to the corresponding average measured concentrations. Tables 5.5 and 5.6 present the concentrations of $Z_{b h}, Z_{e}$ and $S_{p h b}$ in the Phase 1 BR-AD system estimated using the calibrated models. Percent differences between corresponding simulated and measured values are shown and significant differences, as defined by ttest hypotheses at the $95 \%$ confidence level, are shaded grey. 
Table 5.5 Measured and Simulated $Z_{\mathrm{bh}}, Z_{\mathrm{e}}$ and $S_{\mathrm{phb}}$ in BR WAS

\begin{tabular}{|c|c|c|c|c|c|c|c|c|c|c|}
\hline \multirow{3}{*}{$\begin{array}{l}\text { Para- } \\
\text { meter }\end{array}$} & \multicolumn{4}{|c|}{ Measured } & \multicolumn{3}{|c|}{ BioWin Model } & \multicolumn{3}{|c|}{ ASM3 Model } \\
\hline & \multirow[b]{2}{*}{ Avg. } & \multirow{2}{*}{$\begin{array}{l}\text { Std. } \\
\text { dev. }\end{array}$} & \multicolumn{2}{|c|}{$\%$ of PCOD } & \multirow[b]{2}{*}{ Avg. } & \multirow{2}{*}{$\begin{array}{l}\% \text { of } \\
\text { PCOD }\end{array}$} & \multirow{2}{*}{$\begin{array}{l}\text { Difference } \\
\text { from } \\
\text { Measured (\%) }\end{array}$} & \multirow[b]{2}{*}{ Avg. } & \multirow{2}{*}{$\begin{array}{l}\% \text { of } \\
\text { PCOD }\end{array}$} & \multirow{2}{*}{$\begin{array}{l}\text { Difference } \\
\text { from } \\
\text { Measured (\%) }\end{array}$} \\
\hline & & & Avg. & $\begin{array}{l}\text { Std. } \\
\text { dev. }\end{array}$ & & & & & & \\
\hline PCOD & 4127 & 334 & & & 4315 & & 5 & 4218 & & 2 \\
\hline $\mathrm{Z}_{\mathrm{bh}}$ & 2133 & 73 & 52 & 5 & 3452 & 80 & 62 & 2109 & 50 & 1 \\
\hline $\mathrm{Z}_{\mathrm{e}}$ & 512 & 17 & 12 & 1 & 863 & 20 & 69 & 548 & 13 & 7 \\
\hline$S_{\mathrm{phb}}$ & 1543 & 205 & 37 & 6 & 0 & 0 & 100 & 1561 & 37 & 1 \\
\hline
\end{tabular}

Concentrations in $\mathrm{mg} / \mathrm{L}$

Table 5.6 Measured and Simulated $Z_{\mathrm{bh}}, Z_{\mathrm{e}}$ and $S_{\mathrm{phb}}$ in Phase 1 AD WAS

\begin{tabular}{|c|c|c|c|c|c|c|c|c|c|c|}
\hline \multirow{3}{*}{$\begin{array}{l}\text { Para- } \\
\text { meter }\end{array}$} & \multicolumn{4}{|c|}{ Measured } & \multicolumn{3}{|c|}{ BioWin Model } & \multicolumn{3}{|c|}{ ASM3 Model } \\
\hline & \multirow[b]{2}{*}{ Avg. } & \multirow[b]{2}{*}{$\begin{array}{l}\text { Std. } \\
\text { dev. }\end{array}$} & \multicolumn{2}{|c|}{ \% of PCOD } & \multirow[b]{2}{*}{ Avg. } & \multirow{2}{*}{$\begin{array}{l}\% \text { of } \\
\text { PCOD }\end{array}$} & \multirow{2}{*}{$\begin{array}{l}\text { Difference } \\
\text { from } \\
\text { Measured (\%) }\end{array}$} & \multirow[b]{2}{*}{ Avg. } & \multirow{2}{*}{$\begin{array}{l}\% \text { of } \\
\text { PCOD }\end{array}$} & \multirow{2}{*}{$\begin{array}{l}\text { Difference } \\
\text { from } \\
\text { Measured (\%) }\end{array}$} \\
\hline & & & Avg. & $\begin{array}{l}\text { Std. } \\
\text { dev. }\end{array}$ & & & & & & \\
\hline PCOD & 2119 & 218 & & & 2208 & & 4 & 2142 & & 5 \\
\hline $\mathrm{Z}_{\mathrm{bh}}$ & 1200 & 260 & 57 & 11 & 1281 & 58 & 7 & 1116 & 52 & 7 \\
\hline $\mathrm{Z}_{\mathrm{e}}$ & 1090 & 350 & 51 & 17 & 927 & 42 & 15 & 982 & 46 & 10 \\
\hline $\mathrm{S}_{\mathrm{phb}}$ & 0 & 0 & 0 & 0 & 0 & 0 & 0 & 44 & 2 & 100 \\
\hline
\end{tabular}

Concentrations in $\mathrm{mg} / \mathrm{L}$

The BioWin model could not be calibrated to predict $Z_{b h}, Z_{e}$ and $S_{\text {phb }}$ concentrations in the BR WAS that were statistically equivalent to the respective average measured concentrations while also satisfying the condition that the predicted PCOD concentrations in the BR and AD WAS be statistically equivalent to the respective average measured concentrations. The offline respirometric measurements indicated that a portion of the synthetic feed remained stored inside the BR biomass. The presence of stored COD resulted in less grown biomass and hence less decay of biomass to produce $\mathrm{Z}_{\mathrm{e}}$. However, the BioWin model assumed that the COD of the synthetic feed was completely oxidized by the BR biomass with the SRT employed. Therefore, the model overestimated the $\mathrm{Z}_{\mathrm{bh}}$ and $\mathrm{Z}_{\mathrm{e}}$ concentrations in the BR WAS when compared to the respective measured concentrations. The BioWin model was successfully calibrated in terms of predicting $Z_{\mathrm{bh}}, \mathrm{Z}_{\mathrm{e}}$ and $\mathrm{S}_{\mathrm{phb}}$ concentrations in the AD WAS that were statistically equivalent to the corresponding measured concentrations and this was because the measured $S_{\text {phb }}$ concentration in the AD WAS was negligible.

The ASM3 Model was successfully calibrated in terms of predicting $Z_{\mathrm{bh}}$ and $\mathrm{S}_{\mathrm{phb}}$ concentrations in the BR WAS that were statistically equivalent to the respective measured concentrations. This model therefore successfully accounted for the presence of stored COD in the BR WAS. The $\mathrm{Z}_{\mathrm{e}}$ 
concentration in the BR WAS predicted by this model was significantly higher than the measured concentration although the difference was $7 \%$ which was considered to be relatively small. The ASM3 Model was also successfully calibrated in terms of predicting $Z_{b h}$ and $Z_{e}$ concentrations in the Phase 1 AD WAS that were statistically equivalent to the respective measured concentrations. The calibrated ASM3 Model predicted that $44 \mathrm{mg} / \mathrm{L}$ of $\mathrm{S}_{\mathrm{phb}}$ remained in the AD WAS whereas the measurements indicated that $S_{\text {phb }}$ was fully depleted in the AD. However, this predicted $S_{\text {phb }}$ concentration was only $2 \%$ of the PCOD and hence was considered negligible.

Overall, the $\mathrm{Z}_{\mathrm{bh}}, \mathrm{S}_{\mathrm{phb}}$ and $\mathrm{Z}_{\mathrm{e}}$ concentrations in the BR and AD WAS predicted by the calibrated ASM3 Model were deemed to be comparable to those estimated from the offline respirometric measurements despite the fact that the modeling and respirometric approaches employed different values of $Y_{h}, b_{h}$ and $\mu_{\mathrm{h}}$. In the calibrated ASM3 Model, the values of $\mathrm{Y}_{\mathrm{STO}, \mathrm{O} 2}, \mathrm{Y}_{\mathrm{H}, \mathrm{O} 2}, \mathrm{~b}_{\mathrm{H}, \mathrm{O} 2}$ and $\mu_{\mathrm{h}}$ were $0.8,0.8,0.3 \mathrm{~d}^{-1}$ and $0.56 \mathrm{~d}^{-1}$ at $20^{\circ} \mathrm{C}$, respectively. The net yield of heterotrophic biomass $\left(\mathrm{Y}_{\mathrm{h}}\right)$ was the product of $\mathrm{Y}_{\mathrm{STO}, \mathrm{O} 2}$ and $\mathrm{Y}_{\mathrm{H}, \mathrm{O} 2}$, i.e. 0.64. By comparison, equations 2.3 and 4.15 used to estimate $\mathrm{Z}_{\mathrm{bh}}$ and $\mathrm{Z}_{\mathrm{e}}$ from respirometry employed $Y_{h}, b_{h}$ and muh values of $0.6,0.24 \mathrm{~d}^{-1}$ and $3 \mathrm{~d}^{-1}$, respectively.

In addition to predicting particulate COD concentrations in the reactors that were statistically equivalent to the respective measured concentrations, the calibration of the models was also based on minimizing the sum of squared differences between the measured and predicted OUR responses from offline respirometry on the BR WAS. Figure 5.2 shows the OUR responses in the BR WAS measured by offline respirometry and simulated by the calibrated models. The measured OUR curve in Figure 5.2 was typical of that obtained throughout Phases 1 and 2.

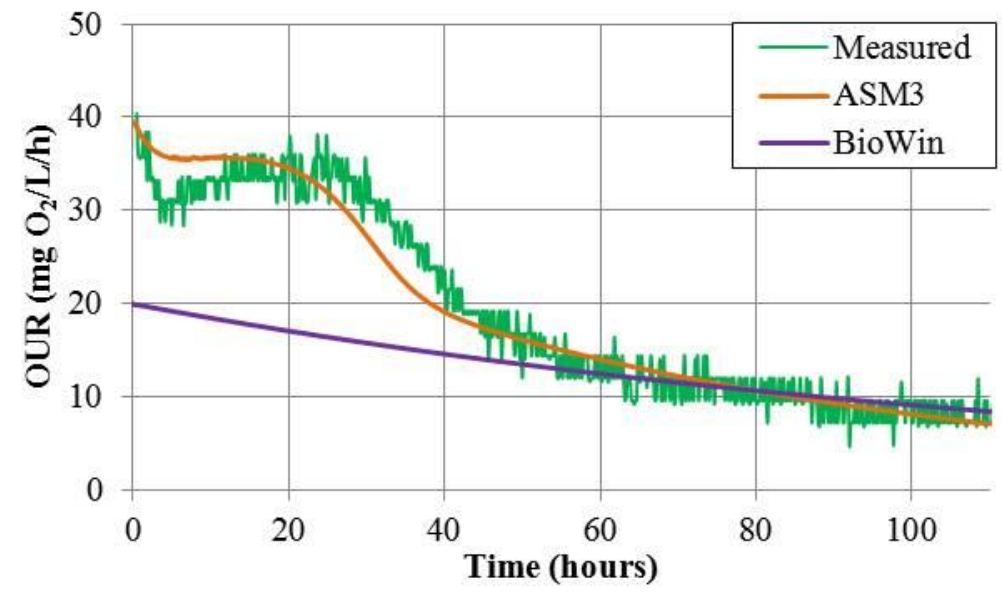

Figure 5.2 Measured and Simulated OUR from Offline Respirometry on BR WAS 
From Figure 5.2 it can be seen that the measured OUR curve initially declined, peaked and then decreased exponentially which suggested that biomass growth, COD oxidation and endogenous respiration occurred during the test. The calibrated BioWin Model successfully predicted only the endogenous response, i.e. from 55 hours onwards. It was not possible to adjust the kinetic or stoichiometric parameters in the BioWin Model to simulate the growth or COD oxidation observed in the batch test because this model did not include processes for COD storage by $\mathrm{Z}_{\mathrm{bh}}$. The BioWin Model was therefore deficient in this regard. Although the OUR curve predicted by the calibrated ASM3 Model did not demonstrate a peak, it did plateau and then decrease exponentially which suggested that COD oxidation and endogenous respiration occurred during the test. These results and the predicted and measured $Z_{b h}, Z_{e}$ and $S_{\text {phb }}$ concentrations in the BR WAS, previously presented in Table 5.5, indicate that the calibrated ASM3 Model was better suited to simulate the BR-AD system than the calibrated BioWin Model. Therefore, the calibrated ASM3 Model was selected to subsequently simulate the Phase 1 and 2 systems.

Using the calibrated ASM3 Model, the measured and simulated OUR responses in the batch respirometric tests of inoculated BR WAS and AD WAS were subsequently compared, as shown in Figure 5.3. The model successfully predicted the OUR response due to oxidation of stored COD and endogenous respiration in the inoculated BR WAS. The model also correctly predicted the endogenous OUR response in the AD WAS. The apparent success of these two predicted responses was considered to validate the calibration of the ASM3 Model since these responses were independent from the calibration.

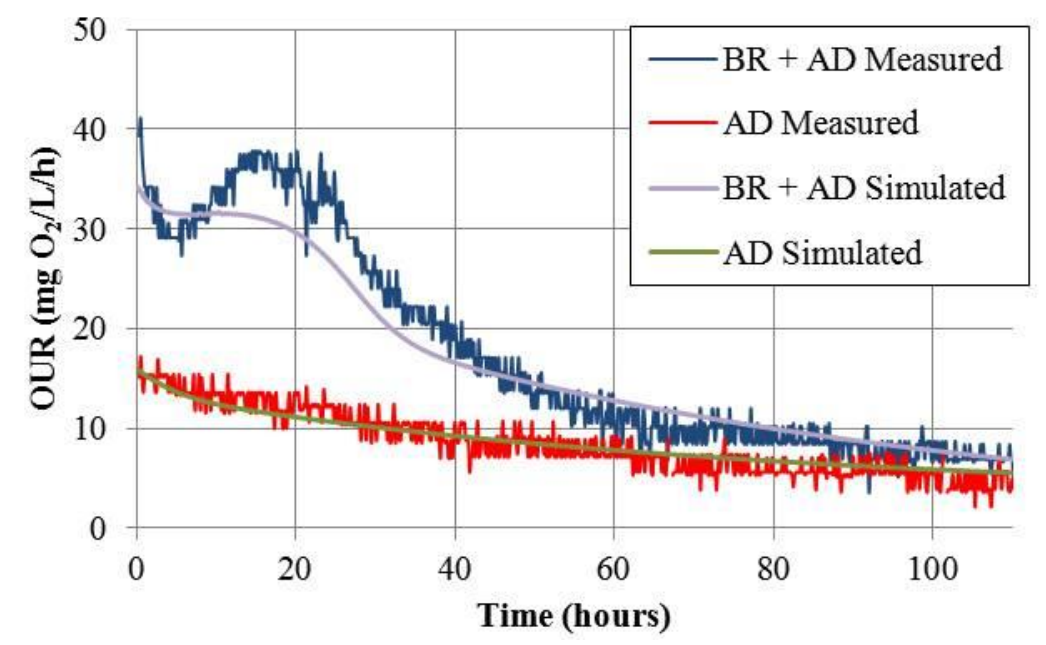

Figure 5.3 Measured and Simulated OUR from Offline Respirometry on Inoculated BR WAS and AD WAS 
The OUR response in the AD simulated by the calibrated ASM3 Model was compared to the OUR response measured by online respirometry during Phase 1, as shown in Figure 5.4. As mentioned in section 4.3.1, there was only one viable online respirometric test in Phase 1.

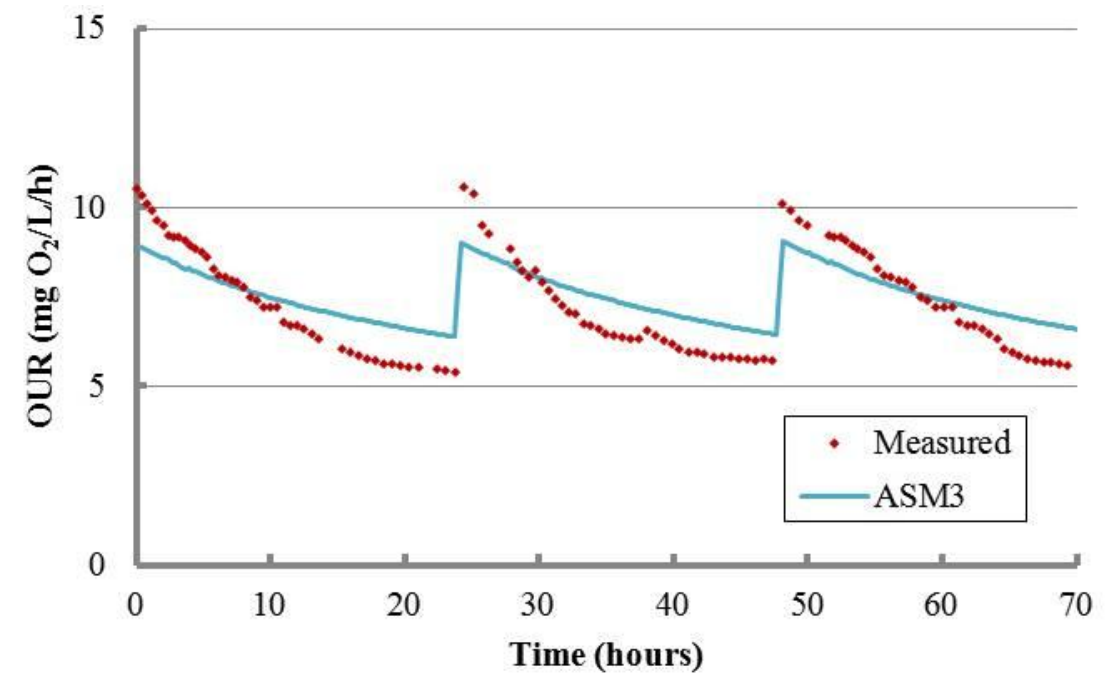

Figure 5.4 Measured and Simulated OUR from Online Respirometry in Phase 1 AD

Similar to the measured OUR curve in each reaction period, the simulated curves in Figure 5.4 appeared to be decay-dominated. The areas under the predicted and measured OUR curves in each reaction period were comparable which indicated that the predicted and measured total oxygen uptake in each reaction period were comparable. However, the simulated OUR curve somewhat underestimated the OUR immediately after feeding and somewhat overestimated the OUR over the second half of the reaction period. As shown in Figure 5.4, the observed OUR in the Phase 1 AD immediately after feeding was $10.5 \mathrm{mgO}_{2} / \mathrm{L} / \mathrm{h}$, and this decreased over the reaction period to 5.5 $\mathrm{mgO}_{2} / \mathrm{L} / \mathrm{h}$. By comparison, the OUR in the AD predicted by the calibrated ASM3 Model was 8.9 $\mathrm{mgO}_{2} / \mathrm{L} / \mathrm{h}$ immediately feeding, and this decreased over the reaction period to $6.4 \mathrm{mgO}_{2} / \mathrm{L} / \mathrm{h}$. Since there was only one viable measured response, additional experiments should be carried out to further assess the extent to which the OUR response predicted by the calibrated ASM3 Model may deviate from the measured OUR response and investigate the possible causes of this deviation. 


\subsection{Phase 2 System Modeling}

The calibrated ASM3 Model that was used to simulate the Phase 1 system was subsequently employed to simulate the Phase 2 system with the same kinetic and stoichiometric parameter values. Once the simulated Phase 1 system was at steady state, the feed to the AD was switched from BR WAS to an influent intended to represent the pretreated BR WAS, labeled "PT" in Figure 5.5. The simulation was continued for a number of days until a new steady state condition was reached. The configuration of this switchover from Phase 1 to 2 in the BioWin 3.1® platform is shown in Figure 5.5 .

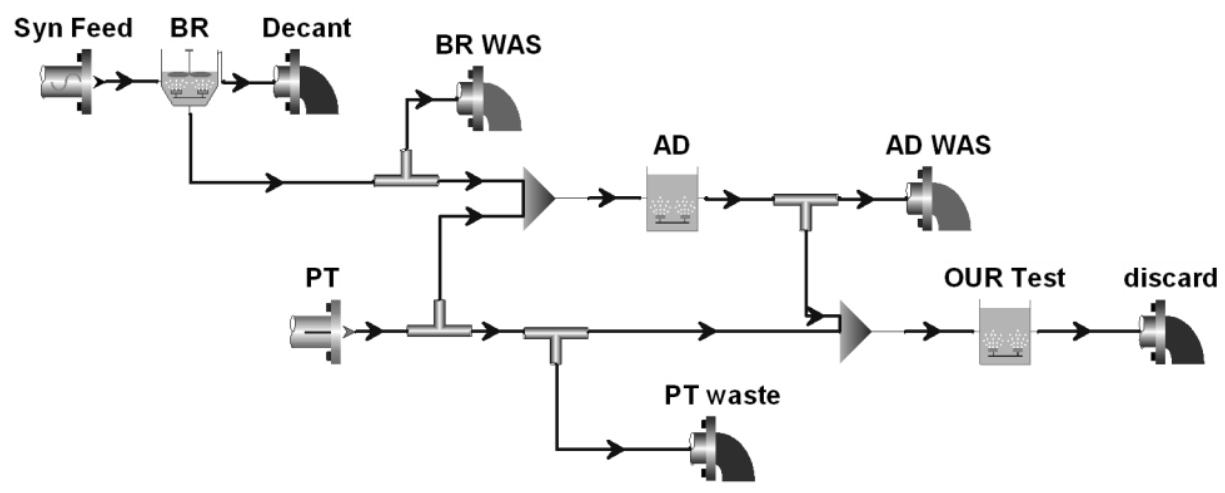

Figure 5.5 Configuration of Switchover from Phase 1 to 2 in BioWin ${ }^{\circledR} 3.1$ Platform

As discussed in section 5.2, the predicted steady-state concentrations of the COD species in the BR WAS were found to be statistically equivalent to the respective average measured concentrations except for $\mathrm{Z}_{\mathrm{e}}$ which was $7 \%$ higher than the measured concentration. However, this $7 \%$ difference was considered relatively small hence the predicted composition of the BR WAS was deemed representative of the measured composition. The simulated BR WAS contained $53 \mathrm{mg} / \mathrm{L} \mathrm{S}_{\mathrm{us}}, 2109$ $\mathrm{mg} / \mathrm{L} \mathrm{Z} \mathrm{Z}_{\mathrm{bh}}, 548 \mathrm{mg} / \mathrm{L} \mathrm{Z}_{\mathrm{e}}$ and $1561 \mathrm{mg} / \mathrm{L} \mathrm{S}_{\mathrm{phb}}$. Thus the simulated TCOD concentration was 4271 $\mathrm{mg} / \mathrm{L}$.

The simulated composition of the BR WAS was used to estimate several properties of the pretreated BR WAS, as follows:

- The simulated pretreated BR WAS was assigned the same concentrations of ISS, phosphate, magnesium, calcium, alkalinity, cations and anions as were in the simulated BR WAS.

- The TCOD concentration in the pretreated BR WAS was assumed to be equivalent to that in the BR WAS because it was demonstrated in section 4.2.3 that TCOD was conserved during pretreatment. 
- The $\mathrm{S}_{\text {us }}$ concentration in the simulated pretreated BR WAS was assumed to be equivalent to that in the BR WAS because it was shown in section 4.2.4 that pretreatment did not generate this type of COD.

- The $X_{\mathrm{i}}$ concentration in the pretreated BR WAS was assumed to be equivalent to that in the BR WAS, i.e. the concentration of endogenous residue in the BR WAS, because it was shown in section 4.2.4 that pretreatment did not alter the biodegradable fraction of the WAS.

- The $S_{\text {phb }}$ concentration in the BR WAS was assumed to be fully converted to $S_{\text {bsc }}$ by pretreatment because it was assumed that the storage products in the BR WAS were released upon cell inactivation by pretreatment.

- The $\mathrm{Z}_{\mathrm{bh}}$ in the BR WAS was assumed to be converted to a mixture of $\mathrm{S}_{\mathrm{bsc}}$ and $\mathrm{X}_{\mathrm{sp}}$ by pretreatment.

Therefore, the simulated pretreated BR WAS contained $53 \mathrm{mg} / \mathrm{L} \mathrm{S} \mathrm{us}_{3}, 548 \mathrm{mg} / \mathrm{L} \mathrm{Z}$ and the remaining $3670 \mathrm{mg} \mathrm{COD} / \mathrm{L}$ was assumed to be bCOD comprised of a mixture of $\mathrm{S}_{\mathrm{bsc}}$ and $\mathrm{X}_{\mathrm{sp}}$. Thus the bCOD comprised $86 \%$ of the TCOD of the pretreated BR WAS.

The measured concentrations of nitrogen species presented in chapter 4 were used to estimate the nitrogen species in the simulated pretreated WAS. As shown in section 4.2.3, pretreatment solubilized organic nitrogen but did not change the ammonia concentration in the WAS. Therefore the same ammonia concentration predicted in the BR WAS was assumed to be present in the pretreated BR WAS, i.e. $70 \mathrm{mg}$ N/L. The particulate organic nitrogen $\left(\mathrm{X}_{\mathrm{on}}\right)$ concentration in the pretreated BR WAS was assumed to be the product of the typical nitrogen content of biomass (0.07 $\mathrm{mg} \mathrm{N} / \mathrm{mg}$ COD) and the sum of $X_{\mathrm{sp}}$ and $X_{\mathrm{i}}$ since $X_{\mathrm{sp}}$ originated from biomass and $X_{\mathrm{i}}$ was comprised of endogenous residue. Similarly, the soluble organic nitrogen $\left(\mathrm{N}_{\mathrm{os}}\right)$ concentration in the pretreated BR WAS was assumed to be the product of the typical nitrogen content of biomass $(0.07 \mathrm{mg} \mathrm{N} / \mathrm{mg}$ $\mathrm{COD}$ ) and the $\mathrm{S}_{\mathrm{bsc}}$ concentration in the pretreated BR WAS that originated from $\mathrm{Z}_{\mathrm{bh}}$ in the BR WAS. The $S_{\text {bsc }}$ in the pretreated BR WAS that originated from $S_{\text {phb }}$ in the BR WAS was assumed to be absent of nitrogen since glycogen, the likely storage product, does not contain nitrogen.

The Phase 2 system was simulated as described above for various concentrations of $S_{\mathrm{bsc}}$ and $\mathrm{X}_{\mathrm{sp}}$ in the pretreated BR WAS while maintaining a bCOD concentration of $3670 \mathrm{mg} / \mathrm{L}$ and a minimum $\mathrm{S}_{\mathrm{bsc}}$ concentration of $1561 \mathrm{mg} / \mathrm{L}$, i.e. the concentration of $S_{\mathrm{bsc}}$ originating from $S_{\mathrm{phb}}$ in the BR WAS. A trial and error approach was used to initially estimate the $S_{\mathrm{bsc}}$ and $X_{\mathrm{sp}}$ concentrations such that the predicted concentrations of PCOD and SCOD in the pretreated BR WAS and PCOD in the Phase 2 AD WAS were statistically equivalent to the respective measured concentrations using t-tests at the 
95\% confidence level. Following this, a least squares regression between the predicted and measured OUR responses from offline respirometry on the inoculated pretreated BR WAS was employed to improve the accuracy of the $S_{b s c}$ and $X_{s p}$ estimates. Based on this approach the concentrations of $S_{b s c}$ and $X_{\mathrm{sp}}$ in the pretreated BR WAS were estimated to be $2316 \mathrm{mg} / \mathrm{L}$ and $1354 \mathrm{mg} / \mathrm{L}$. Table 5.7 presents the simulated and measured PCOD and SCOD concentrations in the pretreated BR WAS.

Table 5.7 Measured and Simulated COD Species in Pretreated BR WAS

\begin{tabular}{|c|c|c|c|c|c|c|}
\hline \multirow{2}{*}{ Parameter } & \multicolumn{2}{|c|}{ Measured } & \multicolumn{4}{|c|}{ ASM3 Model } \\
\hline & Avg. & Std. dev. & Parameter & Pred & & Difference from Measured (\%) \\
\hline \multirow{2}{*}{ PCOD } & \multirow{2}{*}{1773} & \multirow{2}{*}{264} & $\mathrm{X}_{\mathrm{sp}}$ & 1354 & \multirow{2}{*}{1902} & \multirow{2}{*}{7} \\
\hline & & & $\mathrm{Z}_{\mathrm{e}}$ & 548 & & \\
\hline \multirow{2}{*}{ SCOD } & \multirow{2}{*}{2369} & \multirow{2}{*}{228} & $\mathrm{~S}_{\mathrm{bsc}}$ & 2316 & \multirow{2}{*}{2369} & \multirow{2}{*}{0} \\
\hline & & & $\mathrm{S}_{\mathrm{us}}$ & 53 & & \\
\hline
\end{tabular}

Concentrations in $\mathrm{mg} / \mathrm{L}$

In Table 5.7 the predicted PCOD concentration was the sum of the predicted $\mathrm{X}_{\mathrm{sp}}$ and $\mathrm{Z}_{\mathrm{e}}$ concentrations. Although the predicted PCOD concentration was $7 \%$ higher than the average measured concentration, this difference was insignificant at the $95 \%$ confidence level. The predicted SCOD concentration, which was the sum of the predicted $S_{\text {bsc }}$ and $S_{\text {us }}$ concentrations, was identical to the average measured SCOD concentration.

Employing $\mathrm{S}_{\mathrm{bsc}}$ and $\mathrm{X}_{\mathrm{sp}}$ estimates of $2316 \mathrm{mg} / \mathrm{L}$ and $1354 \mathrm{mg} / \mathrm{L}$, respectively, in the pretreated BR WAS also resulted in the predicted PCOD concentration in the Phase 2 AD WAS being statistically equivalent to the average measured concentration. The predicted and measured PCOD concentrations in the Phase 2 AD WAS are shown in Table 5.8. In addition, Table 5.8 compares the predicted and measured SCOD, ffCOD, VSS, ISS, $\mathrm{NO}_{3}-\mathrm{N}$ and $\mathrm{NH}_{3}-\mathrm{N}$ concentrations. Percent differences between corresponding simulated and measured values were calculated and significant differences, determined by t-test hypotheses at the $95 \%$ confidence level, are shaded grey in Table 5.8. 
Table 5.8 Measured and Simulated COD, SS and N Species in Phase 2 AD WAS

\begin{tabular}{|l||l|l||l|l|}
\hline \multirow{2}{*}{ Parameter } & \multicolumn{2}{|c|}{ Measured } & \multicolumn{2}{c|}{ ASM3 Model } \\
\cline { 2 - 5 } & Avg. & Std. dev. & Predicted & $\begin{array}{l}\text { Difference from Measured } \\
(\boldsymbol{\%})\end{array}$ \\
\hline PCOD & 1861 & 67 & 1898 & 2 \\
\hline SCOD & 237 & 31 & 53 & 78 \\
\hline ffCOD & 177 & 31 & 53 & 70 \\
\hline VSS & 1557 & 90 & 1518 & 3 \\
\hline ISS & 528 & 44 & 732 & 39 \\
\hline NO3-N & 0.9 & 0.9 & 0 & 100 \\
\hline NH3-N & 112 & 9 & 113 & 1 \\
\hline
\end{tabular}

Concentrations in $\mathrm{mg} / \mathrm{L}$

As shown in Table 5.8, the model predicted VSS and $\mathrm{NH}_{3}-\mathrm{N}$ concentrations in the Phase 2 AD that were statistically equivalent to the respective measured concentrations. It is believed that this finding validated the estimation of the $S_{\mathrm{bsc}}$ and $\mathrm{X}_{\mathrm{sp}}$ concentrations in the pretreated BR WAS since the VSS and $\mathrm{NH}_{3}-\mathrm{N}$ responses were independent of the calibration responses. The predicted $\mathrm{NO}_{3}-\mathrm{N}$ concentration significantly differed from the measured values however both the predicted and measured concentrations were very close to zero and therefore considered negligible. As expected, the model did not predict the generation of $S_{u s}$ in the AD hence the simulated SCOD and ffCOD concentrations were significantly lower than measured. Also as expected, the model did not accurately predict the concentration of ISS in the AD.

Table 5.9 compares the steady-state concentrations of $Z_{b h}, Z_{e}$ and $S_{p h b}$ in the Phase 2 AD WAS estimated from offline respirometric measurements to the respective simulated concentrations. The simulated concentrations were determined using the calibrated ASM3 Model with $S_{\mathrm{bsc}}$ and $\mathrm{X}_{\mathrm{sp}}$ estimates of $2316 \mathrm{mg} / \mathrm{L}$ and $1354 \mathrm{mg} / \mathrm{L}$, respectively, in the pretreated BR WAS. Percent differences between corresponding simulated and measured values were calculated. T-test hypotheses at the $95 \%$ confidence level were used to determine significant differences and these are shaded grey in Table 5.9 . 
Table 5.9 Measured and Simulated $Z_{\mathrm{bh}}, Z_{\mathrm{e}}$ and $S_{\mathrm{phb}}$ in Phase 2 AD WAS

\begin{tabular}{|l||l|l|l|l||l|l|l|}
\hline \multirow{2}{*}{ Parameter } & \multicolumn{4}{c||}{ Measured } & \multicolumn{4}{|c|}{ ASM3 Model } \\
\cline { 2 - 5 } & Avg. & Std. dev. & \% of PCOD & \multirow{2}{*}{ Avg. } & \% of PCOD & $\begin{array}{l}\text { Difference from } \\
\text { Measured (\%) }\end{array}$ \\
\hline & & & Avg. & Std. dev. & & & 2 \\
\hline PCOD & 1861 & 67 & & & 1898 & & 2 \\
\hline$Z_{\mathrm{bh}}$ & 700 & 150 & 38 & 8 & 833 & 44 & 19 \\
\hline$Z_{\mathrm{e}}$ & 850 & 270 & 46 & 15 & 1017 & 54 & 20 \\
\hline $\mathrm{S}_{\mathrm{phb}}$ & 0 & 0 & 0 & 0 & 48 & 2 & 100 \\
\hline
\end{tabular}

Concentrations in $\mathrm{mg} / \mathrm{L}$

The model predicted that $2 \%$ of the PCOD of the Phase 2 AD WAS consisted of $\mathrm{S}_{\text {phb }}$ whereas the batch respirometric measurements indicated that $S_{\text {phb }}$ was absent. However, this predicted level was relatively small when compared to the uncertainty associated with the test method and therefore considered negligible. The predicted concentrations of $\mathrm{Z}_{\mathrm{bh}}$ and $\mathrm{Z}_{\mathrm{e}}$ were significantly different from the respective measured concentrations. As mentioned in section 4.3.2.4, the sum of the measured COD fractions in the Phase 2 AD WAS was significantly less than the measured TCOD of the WAS, resulting in a significant COD closure error. This indicated that there were inaccuracies in the estimates of $Z_{b h}$ and $Z_{e}$ in the Phase 2 AD WAS based on the batch respirometric measurements. The predicted $Z_{b h}$ and $Z_{e}$ concentrations were believed to be more accurate because the predicted PCOD and VSS concentrations were statistically equivalent to the respective average measured levels. Although the $\mathrm{Z}_{\mathrm{bh}}$ concentration in the Phase 2 AD WAS predicted by the calibrated ASM3 Model was $19 \%$ higher than the measured concentration, it was still significantly less than either the measured or predicted $\mathrm{Z}_{\mathrm{bh}}$ concentration in the Phase 1 AD WAS. This finding was consistent with the observed OUR responses in batch respirometry bottles containing AD WAS.

In addition to matching predicted and measured COD concentrations in the pretreated BR WAS and Phase $2 \mathrm{AD}$ WAS, the estimation of the $\mathrm{S}_{\mathrm{bsc}}$ and $\mathrm{X}_{\mathrm{sp}}$ concentrations in the pretreated BR WAS was also based on minimizing the sum of squared differences between the predicted and measured OUR responses from offline respirometry on inoculated pretreated BR WAS. These predicted and measured OUR responses are shown in Figure 5.6. 


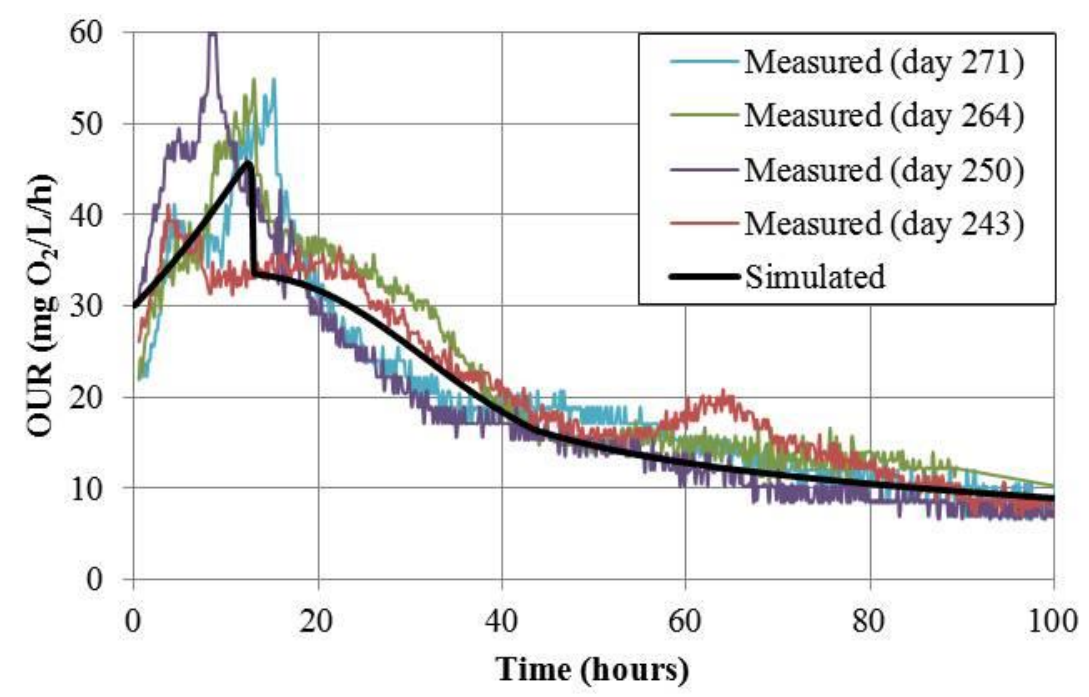

Figure 5.6 Measured and Simulated OUR from Offline Respirometry on Inoculated Pretreated BR WAS

As mentioned in section 4.3.2.2, seven valid offline respirometric measurements were carried out during Phase 2 and an analysis of the results showed four of the tests were conducted with AD biomass that was acclimatized to the pretreated BR WAS. The OUR responses measured during these tests (days 243, 250, 264 and 271) are shown in Figure 5.6. Although there was considerable variability among the four measured responses, each curve showed growth, substrate oxidation and endogenous respiration. Each measured curve showed a sharp peak within the first 20 hours of the test, suggesting growth on $\mathrm{S}_{\mathrm{bsc}}$. Following this, substrate oxidation appeared to dominate the four measured OUR responses until approximately 60 hours. In the tests started on days 250, 264 and 271 , the endogenous response became visible after approximately 60 hours. The measured OUR response from the sample taken on day 243 showed a peak between 60 and 70 hours, suggesting delayed biomass growth. However, the area under this peak comprised only $5 \%$ of the total area under the curve. Therefore, this delayed peak was considered negligible.

A variable volume reactor labeled "OUR Test" was included in the configuration in Figure 5.5 to simulate the offline batch respirometric tests with BR WAS pretreatment. Once the Phase 2 system was at steady-state, $150 \mathrm{~mL}$ of pretreated BR WAS and $50 \mathrm{~mL}$ of AD WAS was fed to the OUR Test reactor and the simulation was continued for a number of days.

In the initial round of calibrations it was found that when the $S_{\mathrm{bsc}}$ to $X_{\mathrm{sp}}$ ratio in the pretreated BR WAS ranged from 1.6 to 1.8 and the bCOD concentration of the pretreated BR WAS was equal to $3670 \mathrm{mg} / \mathrm{L}$, the predicted PCOD and SCOD concentrations in the pretreated BR WAS and predicted 
PCOD concentration in the AD WAS were statistically equivalent to the corresponding measured concentrations. Hence, in the refined calibration, the OUR Test reactor was simulated five times using $\mathrm{S}_{\mathrm{bsc}}$ to $\mathrm{X}_{\mathrm{sp}}$ ratios of $1.60,1.65,1.70,1.75$ and 1.80 . The sum of squared differences was calculated between each of the four measured OUR responses and each of the five simulated OUR responses. For each of the four measured responses, the simulated response that yielded the minimum sum of squared differences was determined. It was found that the best fit estimates of $\mathrm{S}_{\mathrm{bsc}}$ to $\mathrm{X}_{\mathrm{sp}}$ ratios varied over the full extent of the tested range, i.e. 1.6 to 1.8. Therefore, the accuracy of the estimated $S_{b s c}$ and $X_{s p}$ concentrations could not be further improved using this least squares regression approach. The median value of the $S_{\mathrm{bsc}}$ to $\mathrm{X}_{\mathrm{sp}}$ ratio (1.7) was selected and the simulated OUR response associated with this ratio is shown in Figure 5.6. The $S_{\mathrm{bsc}}$ to $\mathrm{X}_{\mathrm{sp}}$ ratio of 1.7 corresponded to the previously mentioned estimated $S_{\mathrm{bsc}}$ and $\mathrm{X}_{\mathrm{sp}}$ concentrations of $2316 \mathrm{mg} / \mathrm{L}$ and $1354 \mathrm{mg} / \mathrm{L}$, respectively. The simulated OUR response appeared to be comprised of two overlapping peaks. An analysis of the modeling results showed that the higher and sharper peak was associated with biomass growth on $\mathrm{S}_{\mathrm{bsc}}$ whereas the lower and more gradual peak was associated with biomass growth on $\mathrm{X}_{\mathrm{sp}}$.

The OUR response in the Phase 2 AD was simulated by the calibrated ASM3 Model with pretreated BR WAS containing $S_{\mathrm{bsc}}$ and $\mathrm{X}_{\mathrm{sp}}$ concentrations of $2316 \mathrm{mg} / \mathrm{L}$ and $1354 \mathrm{mg} / \mathrm{L}$. The simulated response was compared to the OUR response measured by online respirometry, as shown in Figure 5.7. The measured response in Figure 5.7 was typical of the OUR responses measured by online respirometry throughout Phase 2.

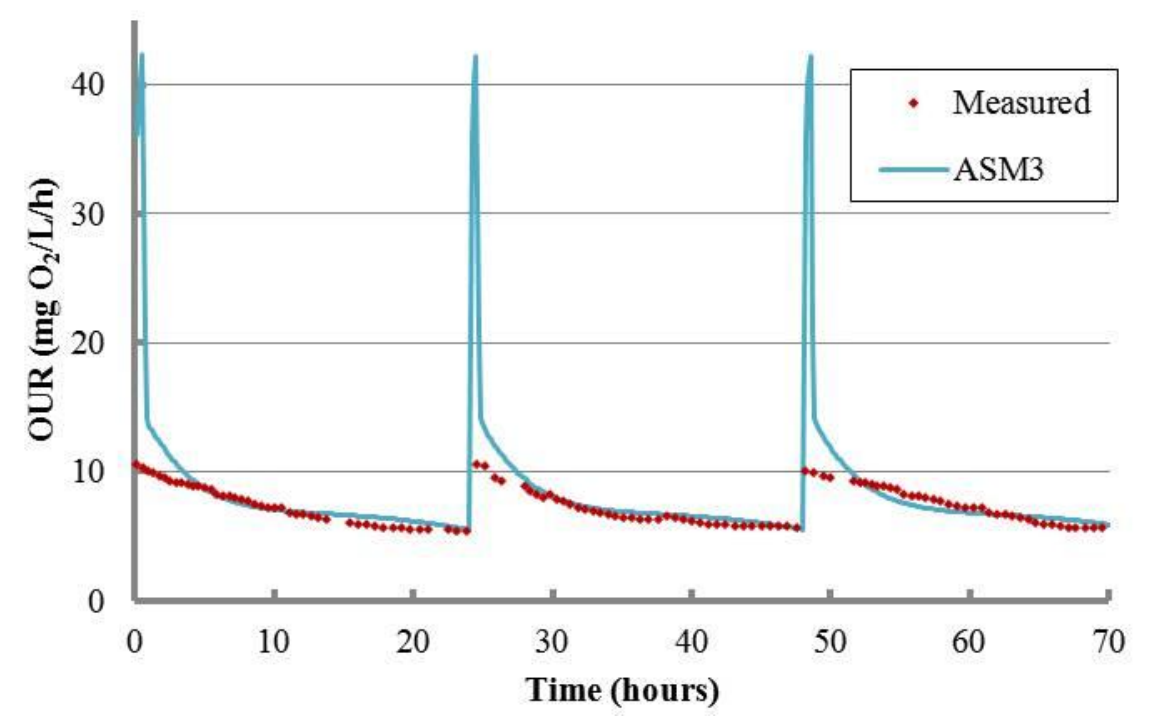

Figure 5.7 Measured and Simulated OUR from Online Respirometry in Phase 2 AD 
As shown in Figure 5.7, the measured OUR in the Phase 2 AD appeared to be decay-dominated as it decreased relatively slowly from $10.5 \mathrm{mgO}_{2} / \mathrm{L} / \mathrm{h}$ immediately after feeding to $5.5 \mathrm{mgO}_{2} / \mathrm{L} / \mathrm{h}$ at the end of the reaction period. By comparison, the simulated OUR in the $\mathrm{AD}$ spiked to $42 \mathrm{mgO} / \mathrm{L} / \mathrm{h}$ immediately after feeding, decreased to $13.5 \mathrm{mgO}_{2} / \mathrm{L} / \mathrm{h}$ within approximately 45 minutes, decreased to $9 \mathrm{mgO}_{2} / \mathrm{L} / \mathrm{h}$ within approximately 3 hours, and then gradually decreased over the reaction period to approximately $5.5 \mathrm{mgO}_{2} / \mathrm{L} / \mathrm{h}$. Although the simulated curve appeared to fit the measured curve from approximately 4 hours after feeding until the end of the reaction period, the simulated curve showed a relatively high spike immediately after feeding that was not observed in the measured response. As explained in section 4.3.2.2, it was hypothesized that the online respirometric measurements failed to capture rapid and high oxygen uptake. Typically 1 hour was required to increase the DO concentration from 3 to $5 \mathrm{ppm}$ immediately after the $\mathrm{AD}$ was fed with pretreated BR WAS. For the remainder of the reaction period, the DO concentration was typically increased from 3 to 5 ppm within 5 minutes. This delay was presumably due to insufficient aeration in the AD. An analysis of the simulated OUR response showed that the spike immediately after feeding was due to the aerobic growth on $S_{\text {bsc. }}$ Apart from this, both the simulated and measured OUR curves appeared to show the oxidation of $\mathrm{X}_{\mathrm{sp}}$, followed by endogenous respiration.

\subsection{Pretreatment Model}

This section describes the approach taken to develop the pretreatment model. The COD fractionation of the WAS before and after pretreatment predicted by the calibrated Phase 2 ASM3 Model was summarized in Table 5.10. The data in Table 5.10 was used to develop a COD-based stoichiometric pretreatment model that is compatible with the Petersen matrix-based models employed in most wastewater treatment simulators, as shown in Table 5.11. Based on the data in Table 5.10, 36\% of $\mathrm{Z}_{\mathrm{bh}}$ in the BR WAS was converted to $\mathrm{S}_{\mathrm{bsc}}$ and the remainder was converted to $\mathrm{X}_{\mathrm{sp}}$. Thus the fraction of $\mathrm{Z}_{\mathrm{bh}}$ converted to $\mathrm{S}_{\mathrm{bsc}}\left(\mathrm{fS}_{\mathrm{bsc}} \mathrm{Z}_{\mathrm{bh}}\right)$ in Table 5.11 was 0.36. As existing simulators do not have the functionality to accommodate instantaneous transformation processes, kinetic processes were employed and the rate constants, $\mathrm{k}_{\mathrm{d} \_} \mathrm{Z}_{\mathrm{bh}}$ and $\mathrm{k}_{\mathrm{d} \_} \mathrm{S}_{\mathrm{phb}}$, were assigned very high values, i.e. $1000 \mathrm{~d}^{-1}$, so that the rates were effectively instantaneous. In accordance with the experimental results, the pretreatment model conserved TCOD and did not generate ammonia. 
Table 5.10 Simulated COD Fractionation of BR WAS and Pretreated BR WAS

\begin{tabular}{|l|l|l||l|l|l|}
\hline BR WAS & $\begin{array}{l}\text { COD } \\
(\mathbf{m g} / \mathbf{L})\end{array}$ & $\begin{array}{l}\text { COD } \\
\text { Fraction (\%) }\end{array}$ & Pretreated WAS & $\begin{array}{l}\text { COD } \\
(\mathbf{m g} / \mathbf{L})\end{array}$ & $\begin{array}{l}\text { COD } \\
\text { Fraction (\%) }\end{array}$ \\
\hline $\mathrm{S}_{\mathrm{phb}}$ & 1561 & 37 & $\mathrm{~S}_{\mathrm{bsc}}\left(\right.$ from $\left.\mathrm{S}_{\mathrm{phb}}\right)$ & 1561 & 36 \\
\hline $\mathrm{Z}_{\mathrm{bh}}$ & 2109 & 49 & $\mathrm{~S}_{\mathrm{bsc}}\left(\right.$ from $\left.\mathrm{Z}_{\mathrm{bh}}\right)$ & 755 & 18 \\
\cline { 3 - 6 } & & $\mathrm{X}_{\mathrm{sp}}$ & 1354 & 32 \\
\hline $\mathrm{Z}_{\mathrm{e}}$ & 548 & 13 & $\mathrm{Z}_{\mathrm{e}}$ & 548 & 13 \\
\hline $\mathrm{S}_{\mathrm{us}}$ & 53 & 1 & $\mathrm{~S}_{\mathrm{us}}$ & 53 & 1 \\
\hline TCOD & 4271 & 100 & TCOD & 4271 & 100 \\
\hline
\end{tabular}

Table 5.11 Pretreatment Model

\begin{tabular}{|l|c|c|c|c|c|}
\hline \multirow{2}{*}{ Process } & $\mathbf{S}_{\mathbf{b s c}}$ & $\mathbf{X}_{\mathbf{s p}}$ & $\mathbf{Z}_{\mathbf{b h}}$ & $\mathbf{S}_{\mathbf{p h b}}$ & \multirow{2}{*}{$\begin{array}{c}\text { Kinetic Rate } \\
\text { Expressions }\end{array}$} \\
\cline { 2 - 5 } Conversion of Heterotrophs & \multicolumn{3}{|c|}{$\mathbf{m g} \mathbf{C O D} / \mathbf{L}$} & \multirow{2}{|c|}{$k_{d-} Z_{b h} \times Z_{b h}$} \\
\hline Release of Stored COD & 1 & & & -1 & $k_{d-} S_{p h b} \times S_{p h b}$ \\
\hline
\end{tabular}

To verify the model, a reactor was added directly upstream of the aerobic digester in the configuration presented in Figure 5.1. This reactor was specified as a BioWin model builder unit with the pretreatment model presented in Table 5.11 to simulate the pretreatment dose employed in this study. The overall system was simulated with the calibrated ASM3 Model that was fit in Phase 1 and the simulations were continued until the $\mathrm{AD}$ reached steady state. The configuration of this Phase 2 system is presented in Figure 5.8.

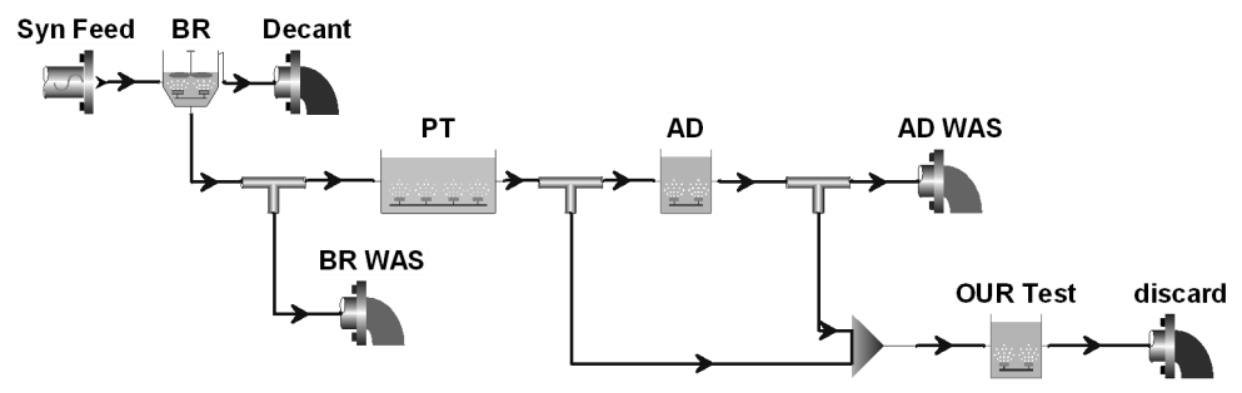

Figure 5.8 Configuration of Phase 2 System in BioWin ${ }^{\circledR}$ 3.1 Platform

The steady-state concentrations of COD, SS, ammonia and nitrate in the Phase 2 AD using the system configuration in Figure 5.8 were identical to the respective concentrations in the Phase 2 AD using the configuration presented previously in Figure 5.5. Likewise, the OUR responses in the online and offline tests simulated using the configuration in Figure 5.8 were identical to those simulated using 
the configuration in Figure 5.5. It was therefore concluded that the pretreatment model presented in Table 5.11 accurately described the COD transformations associated with the HPTH pretreatment dose employed in this study.

The COD-based stoichiometric pretreatment model proposed in Table 5.11 was somewhat specific to the system in this study. The model was based on using the ASM3 Model in the BioWin platform for a non-nitrifying aerobic system treating WAS comprised of $Z_{b h}, Z_{e}$ and $S_{p h b}$. However, the approach described in this study may be followed to determine the impacts of pretreatment on $Z_{b h}, Z_{e}$ and $S_{\text {phb }}$ when other doses of HPTH pretreatment and other pretreatment techniques are employed. It would seem to be reasonable to assume that other types of biomass typically occurring in WWTPs would be undergo similar conversions through pretreatment as was observed for $\mathrm{Z}_{\mathrm{bh}}$ in this study. However, tests should be carried out to verify this. The proposed pretreatment model did not generate ammonia which was in accordance with the experimental results. The model did not account for the possible impacts of pretreatment on phosphorous species since the measurement of these species was outside the scope of this study. 


\section{Conclusions}

This study sought to characterize and develop a COD based model for WAS pretreatment by HPTH and it is believed that all of the objectives were met. The COD of a raw and pretreated synthetically generated WAS was fractionated using analytical and bioassay methods and activated sludge modeling. It was found that HPTH WAS pretreatment at $150^{\circ} \mathrm{C}$ and 3 bars for 30 minutes increased the rate at which the WAS was aerobically biodegraded but did not increase the extent of biodegradation. A COD-based pretreatment model was then developed and the applicability of the model was assessed. The following summarizes the specific conclusions that were made.

In the study it was found that stored COD was present in the synthetically generated (BR) WAS. This conclusion was based on the following independent results:

- The average measured COD/VSS ratio of the BR WAS was $1.23 \pm 0.08$ which was significantly lower than the typical value of 1.42 for active biomass and decay products. This suggested that stored COD such as glycogen was present since it has a relatively low COD/VSS ratio of 1.1 .

- The average measured pON/PCOD ratio of the BR WAS was $0.053 \pm 0.006 \mathrm{mg} \mathrm{N} / \mathrm{mg}$ COD which was significantly lower than the typical value of $0.07 \mathrm{mg} \mathrm{N} / \mathrm{mg}$ COD for active biomass and decay products. Because typical storage products such as glycogen or PHA do not contain nitrogen, this lower measured ratio suggested that storage products were present.

- The average measured ffCOD concentration in the AD WAS was significantly higher than that in the BR WAS which suggested that $S_{\text {us }}$ was generated in the AD. Prior research has shown that the decay of cells storing COD generated $S_{\text {us. }}$. Therefore, the production of $S_{\text {us }}$ in the AD supported the hypothesis that storage products were present in the biomass fed to the $\mathrm{AD}$.

- The OUR responses from offline respirometry on BR WAS showed both growth and decay. It appeared that stored COD was consumed during the batch respirometry and exerted an oxygen demand that was greater than that which would have been attributed to endogenous respiration.

- The BR WAS was estimated to contain $51 \pm 4 \% \mathrm{Z}_{\mathrm{bh}}$ and $12 \pm 1 \% \mathrm{Z}_{\mathrm{e}}$ based on an analysis of the data from the offline respirometric test on mixtures of BR WAS and synthetic feed in high F/M ratios. The calibrated ASM3 Model employed to simulate the Phase 1 BR-AD 
system verified these $Z_{b h}$ and $Z_{e}$ COD fractions in the BR WAS and predicted that the remaining $37 \%$ was essentially comprised of $\mathrm{X}_{\mathrm{STO}}$.

It was concluded that stored COD was not present in the AD WAS. This conclusion was based on two findings: the average measured COD/VSS ratio in the AD WAS was statistically equivalent to the typical ratio for $Z_{b h}$ and $Z_{e}$; and the OUR curves from offline respirometry on AD WAS showed only decay and no growth. Therefore, the stored COD present in the BR WAS was depleted in the AD.

This study showed that HPTH pretreatment at $150^{\circ} \mathrm{C}$ and 3 bars for 30 minutes did not reduce the TCOD concentration of the WAS. This conclusion was based on the finding that the average TCOD concentrations of the raw and pretreated BR WAS were statistically equivalent.

The dose of pretreatment employed in this project did not mineralize organic nitrogen as the average ammonia concentration in the BR WAS did not significantly increase after pretreatment.

HPTH pretreatment at $150^{\circ} \mathrm{C}$ and 3 bars for 30 minutes solubilized $56 \pm 7 \%$ of COD, $49 \% \pm 11 \%$ of organic nitrogen, $56 \pm 10 \%$ of VSS and did not solubilize ISS. All types of particulate organics were solubilized to the same extent by pretreatment as the average measured pON/PCOD ratio of the BR WAS was statistically equivalent to that of the pretreated BR WAS. The observed solubilization was consistent with prior research on HPTH WAS pretreatment.

The soluble COD generated by pretreatment was characterized as $S_{\mathrm{bsc}}$ hence pretreatment increased the rate at which the BR WAS was aerobically degraded. This conclusion was based on three results:

- Pretreatment did not generate $S_{\text {us }}$ because it was shown that the average ffCOD concentrations in the AD WAS in Phases 1 and 2 were statistically equivalent. This finding was consistent with literature reports that have shown that refractory compounds, i.e. $S_{\mathrm{us}}$, were only generated at pretreatment temperatures above $150^{\circ} \mathrm{C}$.

- The offline respirometric tests showed that the OUR curve for inoculated pretreated BR WAS peaked earlier and to a higher value than the OUR curve for the inoculated raw BR WAS. This response was characteristic of growth on $\mathrm{S}_{\mathrm{bsc}}$.

- The offline respirometric tests showed that the OUR in the filtered BR WAS was negligible, indicating the absence of $S_{\text {bsc. }}$ By comparison, the area under the OUR curve for filtered 
pretreated BR WAS contributed more than $60 \%$ of the area under the curve for whole pretreated BR WAS, demonstrating that pretreatment generated a substantial amount of $\mathrm{S}_{\mathrm{bsc}}$.

Although pretreatment increased the rate at which the BR WAS was aerobically biodegraded, it did not increase the extent of biodegradation. The non-biodegradable COD component of the BR WAS, i.e. $\mathrm{Z}_{\mathrm{e}}$, was not converted to biodegradable COD under the pretreatment conditions employed in this study. This conclusion was based on the results of three independent tests:

- The online respirometric data showed that the average $\Sigma \mathrm{OU}_{\mathrm{s}} / \mathrm{TCOD}$ ratios for raw and pretreated BR WAS were statistically equivalent, demonstrating that the extent to which the BR WAS was degraded over the AD reaction period was not increased by pretreating the BR WAS.

- Using the TCOD measurements from the sample bottles of the offline respirometric tests, it was shown that the average $\left(\mathrm{TCOD}_{\mathrm{i}}-\mathrm{TCOD}_{\mathrm{f}}\right) / \mathrm{TCOD}_{\mathrm{i}}$ ratio for BR WAS was statistically equivalent to that for pretreated BR WAS. This indicated that pretreatment did not change the fraction of the BR WAS that could be aerobically degraded by acclimatized inoculum.

- The average measured VSS concentration in the AD WAS was statistically equivalent in Phases 1 and 2. Had pretreatment increased the aerobically biodegradable fraction of the BR WAS, it was expected that the VSS concentration in the AD would have decreased.

A COD-based stoichiometric pretreatment model was developed for HPTH pretreatment at $150^{\circ} \mathrm{C}$ and 3 bars for 30 minutes. When this model was integrated into BioWin, it was able to accurately simulate both the steady state performance of the overall system employed in this study as well as dynamic respirometry results. The experimental results showed that the TCOD of the BR WAS consisted of $51 \% \mathrm{Z}_{\mathrm{bh}}, 12 \% \mathrm{Z}_{\mathrm{e}}$ and $37 \% \mathrm{X}_{\text {Sто }}$ and the pretreated BR WAS consisted of $12 \% \mathrm{Z}_{\mathrm{e}}$ and a negligible amount of $\mathrm{Z}_{\mathrm{bh}}$. The pretreatment model verified these fractions and predicted that the pretreated BR WAS also contained $54 \% \mathrm{~S}_{\mathrm{bsc}}$ and $32 \% \mathrm{X}_{\mathrm{sp}}$. Two kinetic processes with effectively instantaneous rates were included in the pretreatment model: $\mathrm{X}_{\mathrm{STO}}$ was fully converted to $\mathrm{S}_{\mathrm{bsc}}$; and $36 \%$ of $\mathrm{Z}_{\mathrm{bh}}$ was converted $\mathrm{S}_{\mathrm{bsc}}$ and the remaining $\mathrm{Z}_{\mathrm{bh}}$ was converted to $\mathrm{X}_{\mathrm{sp}}$. The approach described in this study may be followed to determine the impacts of pretreatment on $Z_{b h}, Z_{e}$ and $X_{S T O}$ when other doses of HPTH pretreatment and other pretreatment techniques are employed. 


\section{Recommendations}

The experiments and modeling procedures carried out in this study were successfully used to characterize the impacts of HPTH pretreatment on WAS containing $\mathrm{Z}_{\mathrm{bh}}, \mathrm{Z}_{\mathrm{e}}$ and $\mathrm{X}_{\text {STO. Several }}$ recommendations are provided to improve the approach in future studies:

- The rate of oxygen transfer in the AD should be increased such that the time to raise the DO concentration from the low to high set point is less than one minute, especially after the AD is fed. This will allow the online respirometric test to capture rapid and high oxygen uptake. To achieve this, the current aerators should be replaced by aerators with much higher oxygen transfer efficiencies. Alternatively, the OUR in the AD could be reduced by adding the BR WAS over one or two hours instead of all at once.

- Phase 2 should only be commenced after at least two valid offline respirometric tests with reproducible results have been obtained for pretreated BR WAS with non-acclimatized inoculum. This will allow the impacts of biomass adaptation on the aerobic biodegradability of WAS by HPTH pretreatment to be more reliably determined.

- Phosphorous species should be measured in the various sludge streams during Phases 1 and 2 to determine the associated impacts of HPTH pretreatment. The COD: N: P ratio of the synthetic feed employed by the current study was 100: 4.4: 1.5 hence the concentration of monopotassium phosphate in the synthetic wastewater may have been excessively high. Using the measurements of the phosphorous species, a phosphorous balance could be used to determine whether the monopotassium phosphate concentration in the synthetic wastewater should be decreased.

- Storage products in the BR WAS should be eliminated to simplify the COD fractionation of the sludge streams and the modeling of the overall system. Without significant stored COD, it is believed that the system could be successfully simulated with the BioWin $3.1{ }^{\circledR}$ Integrated Model. It was hypothesized that the BR biomass stored COD such as glycogen or PHA due to the alternating anaerobic and aerobic conditions in the reactor. Although the measured DO concentration was above $2 \mathrm{ppm}$ for most of the reaction period, it was close to zero for up to two hours after feeding. Thus the operation of the BR should be changed to eliminate any anaerobic conditions. For example, the synthetic wastewater could be fed to the BR over an extended period of time. If storage products are still detected after improving the BR operation, the cells could be microbiologically examined using a suitable staining procedure to gain a better understanding of the nature of the storage granules. 
The approach described in this project with the improvements recommended above should be applied to the following future studies:

- Investigation of the impacts of HPTH pretreatment on $\mathrm{X}_{\mathrm{i}}$. Instead of synthetic wastewater, the BR-AD system could be fed with screened raw municipal wastewater which would contain $\mathrm{X}_{\mathrm{i}}$. Thus the non-biodegradable particulate COD in each sludge stream would be the sum of $X_{i}$ and $Z_{e}$. Since the constituent concentrations in municipal wastewater vary with time, Phases 1 and 2 would each need to be operated long enough to accurately estimate the COD fractions. Using the VSS measurements and analyses of the online and offline respirometric data, the impact of pretreatment on the extent of aerobic biodegradability of the BR WAS could be determined. A significant increase would suggest that $X_{i}$ was partially converted to bCOD by pretreatment since it was shown that $\mathrm{Z}_{\mathrm{e}}$ is not affected.

- Investigation of the impacts of HPTH pretreatment at temperatures above and below $150^{\circ} \mathrm{C}$ and the development of corresponding pretreatment models. Prior research suggests that the relative proportion of $S_{b s c}$ to $X_{s p}$ in the pretreated WAS may increase with pretreatment temperature however it has also been shown that refractory compounds, i.e. $S_{\text {us }}$, may be generated at temperatures above $150^{\circ} \mathrm{C}$. It should be determined whether other pretreatment temperatures can change the aerobically biodegradable fraction of the WAS.

- Investigation of the impacts of other pretreatment techniques such as sonication and ozonation and the development of COD-based stoichiometric pretreatment models that accurately characterize each technique.

The approach in the current project should be combined with investigations of the impacts of HPTH pretreatment on other biomass fractions besides $\mathrm{Z}_{\mathrm{bh}}$ so that appropriate processes may be added to the pretreatment model. In WWTPs, heterotrophs that accumulate phosphorus and bacteria that oxidize ammonia and nitrite are present in significant quantities in the activated sludge and would likely be affected by HPTH pretreatment. Frigon and Isazadeh (2010) showed that pretreatment with ozone did not reduce the level of each type of biomass to the same degree. Further research is required to determine whether such a trend exists for HPTH pretreatment.

It is recommended that the approach described in this project be paired with an analysis of the impacts of HPTH pretreatment on the rate and extent of anaerobic biodegradability. This is an important consideration since promising HPTH techniques such as CAMBI ${ }^{\mathrm{TM}}$ or Exexlys ${ }^{\mathrm{TM}}$ are used in conjunction with anaerobic digestion. Biochemical methane potential tests could be carried out on samples from the various sludge streams in the system. 


\section{References}

Abu-Org, M. and T. Goss. 2011. Comparing Thermal Hydrolysis Processes $\left(\mathrm{CAMBI}^{\mathrm{TM}}\right.$ and EXELYS $^{\mathrm{TM}}$ ) for Solids Pretreatment Prior to Anaerobic Digestion. Proceedings of the WEFTEC Conference, Los Angeles, California, October.

Appels, L., A. Van Assche, K. Willems, J. Degreve, J. Van Impe, and R. Dewil. 2010. Peracetic Acid Oxidation as an Alternative Pretreatment for the Anaerobic Digestion of Waste Activated Sludge. Biores. Tech. 102:4124-4130.

Bourgrier, C., J. P. Delgenès, and H. Carrère. 2008. Effects of Thermal Treatments on Five Different Waste Activated Sludge Samples Solubilization, Physical Properties and Anaerobic Digestion. Chem. Eng. J. 139:236-244.

Bourgrier, C., J. P. Delgenès, and H. Carrère. 2007. Impacts of Thermal Pre-Treatments on the SemiContinuous Anaerobic Digestion of Waste Activated Sludge. Biochem. Eng. J. 34:20-27.

Braguglia, C. M., G. Mininni, and A. Gianico. 2008. Is Sonication Effective to Improve Biogas Production and Solids Reduction in Excess Sludge Digestion? Water Sci. \& Tech. 57:479483.

Camacho, P., S. Deleris, V. Geaugey, P. Ginestet, E. Paul. 2002. A Comparative Study Between Mechanical, Thermal and Oxidative Disintegration Techniques of Waste Activated Sludge. Water Sci. Technol. 46(10):79-87.

Canadian Council of Ministers of the Environment. 2009. Canada-Wide Strategy for the Management of Municipal Wastewater Effluent. Whitehorse, Yukon Territory.

Chu, C.P., D. J. Lee, C. S. You, and J. H. Tay. 2002. Weak Ultrasonic Pretreatment on Anaerobic Digestion of Flocculated Activated Biosolids. Water Res. 36:2681-2688.

Climent, M., I. Ferrer, M. del Mar Baeza, A. Artola, F. Vazquez, and X. Font. 2007. Effects of Thermal and Mechanical Pretreatments of Secondary Sludge on Biogas Production Under Thermophilic Conditions. Chem. Eng. J. 133:335-345.

Deleris, S. and J. M. Rouston, 2000. Effect of Ozonation on Activated Sludge Solubilization and Mineralization. Ozone Sci. Eng. 22:473-486.

Dhar, B. R., E. Elbeshbishy, H. Hafez, G. Nakhla, and M. B. Ray. 2012. Simulating the Impact of Thermo-Chemical Pretreatment on Continuous Anaerobic Digestion of Municipal Waste Activated Sludge. Proceedings of The Recovery of Value-Added Products Conference, Waterloo, Ontario, August.

a'Donoso-Bravo, A., G. Garcia, S. Perez-Elvira, and F. Fdz-Polanco. 2010. Initial Rates Technique as a Procedure to Predict the Anaerobic Digester Operation. Biochem. Eng. J. 53:275-280. 
${ }^{b}$ Donoso-Bravo, A. S., Perez-Elvira, E. Aymerich, and F. Fdz-Polanco. 2010. Assessment of the Influence of Thermal Pretreatment Time on the Macromolecular Composition and Anaerobic Biodegradability of Sewage Sludge. Biores. Tech. 102:660-666.

Dwyer, J., D. Starrenburg, S. Tait, K. Barr, D. J. Batstone, and P. Lant. 2008. Decreasing Activated Sludge Thermal Hydrolysis Temperature Reduces Product Colour, Without Decreasing Degradability. Water Res. 42:4699-4709.

Eaton, A. D., M. A. H. Franson, American Public Health Association, American Water Works Association, and Water Environment Federation. 2005. Standard Methods for the Examination of Water \& Wastewater, 21st Edition. American Public Health Association. Washington, DC.

EnviroSim Associates Ltd. 2011. Short Course: Calibration of Wastewater Plant Simulators. Hamilton, ON.

Frigon, D., and S. Isazadeh. 2010. Evaluation of a New Model for the Reduction of Excess Sludge Production by Ozonation of Return Activated Sludge: What Solids COD Fraction is Affected? Proceedings of the IWA World Water Congress and Exhibition, Montreal, QC, September.

Gurieff, N., J. Bruss, S. Hoejsgaard, J. Boyd, and M. Kline. 2011. Maximizing Energy Efficiency and Biogas Production: EXELYS - Continuous Thermal Hydrolysis. Proceedings of the WEFTEC Conference, Los Angeles, California, October.

Henze, M., M.C.M. van Loosdrecht, G.A. Ekama, and D. Brdjanovic. 2008. Biological Wastewater Treatment Principles, Modelling and Design. IWA Publishing. London, United Kingdom.

Henze, M., W. Gujer, T. Mino, and M. van Loosdrecht. 2000. Activated Sludge Models ASM1, ASM2, ASM2d and ASM3. IWA Publishing. London, United Kingdom.

Jones, R., W. Parker, H. Zhu, D. Houweling, and S. Murthy. 2009. Predicting the Degradability of Waste Activated Sludge. Water Environ. Res. 81:8:765-771.

Jones, R., W. Parker, Z. Khan, S. Murthy, and M. Rupke. 2008. Characterization of Sludges for Predicting Anaerobic Digester Performance. Water Sci. \& Tech. 721-726.

Kianmehr, P. 2010. Characterization of Pretreatment Impacts on Properties of Waste Activated Sludge and Digestibility. Thesis (PhD). University of Waterloo.

Koch, G., M. Kuhni, W. Gujer, and H. Siegrist. 2000. Calibration and Validation of Activated Sludge Model No. 3 For Swiss Municipal Wastewater. Wat. Res. 34:3580-3590.

Lei, L., B. Johnson, J. Sandino, and D. Whitlock. 2010. A Proposed Approach for Modeling Sludge Reduction Technologies. Proceedings of the WEFTEC Conference, New Orleans, Louisiana, October. 
Li, Y. Y., and T. Noike. 1992. Upgrading of Anaerobic Digestion of Waste Activated Sludge by Thermal Pretreatment. Water Sci. Tech. 26:857-866.

Mathieu, S., and P. Etienne. 2000. Estimation of Wastewater Biodegradable COD Fractions by Combining Respirometric Experiments in Various SO/XO Ratios. Water Res. 34:1246.

Melcer, H., P.L. Dold, R.M. Jones, et al. 2003. Methods for Wastewater Characterization in Activated Sludge Modeling. Water Environment Research Foundation. Alexandria, VA.

MOE, Hydromantis Inc. and XCG Consultants Ltd. 2008. Design Guidelines for Sewage Works. Ministry of the Environment, Ontario.

Mohammadali, M. and E. Hall. 2011. Short Report on the Operating Conditions of UBC Laboratory Scale Conventional Activated Sludge Units. Interim Report to the Canadian Water Network for Project EC - 2. Department of Civil Engineering, University of British Columbia. Vancouver, BC.

Morgan-Sagasume, F., S. Pratt, A. Karlsson, D. Cirne, P. Lant, and A. Werker. 2010. Production of Volatile Fatty Acids by Fermentation of Waste Activated Sludge Pretreated in Full-Scale Thermal Hydrolysis Plants. Biores. Tech. 102:3089-3097.

Musser, J. 2009. Integration of Ozone and Ultrasound Activated Sludge Pretreatments into a Wastewater Treatment Whole-Plant Simulator. Thesis (MASc). University of Waterloo.

Namkung, E., and B. E. Rittmann. 1986. Soluble Microbial Products (SMP) Formation Kinetics by Biofilms. Wat. Res. 20:6:795-806.

Neyens, E., and J. Baeyens. 2003. A Review of Thermal Sludge Pre-treatment Processes to Improve Dewaterability. J. of Hazardous Materials. B98:51-67.

Nielsen, H.B., Z. Mladenovska, P. Westermann, B.K. Ahring. 2004. Comparison of Two-Stage Thermophilic $\left(68^{\circ} \mathrm{C} / 55^{\circ} \mathrm{C}\right)$ Anaerobic Digestion with One-Stage Thermophilic $\left(55^{\circ} \mathrm{C}\right)$ Digestion of Cattle Manure. Biotechnol. Bioeng. 86:291-300.

Parker, W. J. 2010. CIVE 771 Biological Wastewater Treatment: Theory and Practice. Waterloo, ON: University of Waterloo.

Phothilangka, P., M. A. Schoen, and B. Wett. 2008. Benefits and Drawbacks of Thermal PreHydrolysis for Operational Performance of Wastewater Treatment Plants. Water Sci. Technol. 58(8):1547-53.

Prorot, A., J. Laurent, C. Dagot, and P. Leprat. 2011. Sludge Disintegration during Heat Treatment at Low Temperature: A Better Understanding of Involved Mechanisms with a Multiparametric Approach. Biochem. Eng. J. 54:178-184.

Ramdani, A., P. Dold, S. Deleris, D. Lamarre, A. Gadbois, and Y. Comeau. 2010. Biodgradation of the Endogenous Residue of Activated Sludge. Water Res. 44:2179-2188. 
Ramdani, A., P. Dold, A. Gadbois, S. Deleris, D. Houweling, and Y. Comeau. 2012. Characterization of the Heterotrophic Biomass and the Endogenous Residue of Activated Sludge. Water Res. 46:653-668.

Reynolds, T.D. and P. A. Riehards. 1996. Unit Operations and Process in Environmental Engineering, 2nd ed. PWS Publishing. Boston.

Rittmann, B. E., and P.L. McCarty. 2001. Environmental Biotechnology: Principles and Applications. McGraw-Hill. New York.

Saby, S., M. Djafer, and G. H. Chen. 2002. Feasibility of Using a Chlorination Step to Reduce Excess Sludge in Activated Sludge Process. Water Res. 36:656-666.

Shimizu, T., K. Kudo, and Y. Nasu. 1993. Anaerobic Waste Activated Sludge Digestion - A Bioconversion and Kinetic Model. Biotechol. Bioeng. 41:1082-1091.

Spanjers, H., and P. Vanrolleghem. 1995. Respirometry as a Tool for Rapid Characterization of Wastewater and Activated Sludge. Water Res. 31:105-107.

Tanaka, S. and K. Kamiyama. 2002. Thermo-Chemical Pretreatment in the Anaerobic Digestion of Waste Activated Sludge. Water Sci. Technol. 46(10):173-179.

Tattersall, J. M., G. Knight, and B. Ning. 2011. 10 Years of Integrating Thermal Hydrolysis into Biosolids Treatment - What are the Lessons Learned? Proceedings of the WEFTEC Conference, Los Angeles, California, October.

Tchobanoglous, G., F.L. Burton, and H.D. Stensel. 2003. Wastewater Engineering Treatment and Reuse, Fourth Edition. McGraw-Hill. New York.

Tiehm, A., K. Nickel, M. Zellhorn, and U. Neis. 2001. Ultrasonic Waste Activated Sludge Disintegration for Improving Anaerobic Stabilization. J. Water Res. 35(8):2003-2009.

USEPA, 1999. Environmental Regulations and Technology: Control of Pathogens and Vectors in Sewage Sludge. EPA/625/R-92/013.

Wang, Q. H., J. C. Chen, K. Kakimoto, H. I. Ogawa, and Y. Kato. 1995. Pretreatment of Waste Activated Sludge Results in Enhancement of its Anaerobic Digestion Efficiency. Journal of the Society of Water Environ. 18:857-882.

Wang, Q., M. Kuninobu, K. Kakimoto, H. I. Ogawa, and Y. Kato. 1999. Upgrading of Anaerobic Digestion of Waste Activated Sudge by Ultrasonic Pretreatment. Biores. Technol. 68:309313.

Wentzel, M.C., M.F. Ubisi, and G.A. Ekama. 1998. Heterotrophic Active Biomass Component of Activated Sludge Mixed Liquor. Wat. Sci Tech. 37:79-87.

Wilson, C. A., and J. T. Novak. 2009. Hydrolysis of Macromolecular Components of Primary and Secondary Wastewater Sludge by Thermal Hydrolytic Pretreatment. Water Res. 42:4489-98. 
Young, J. C., and R. M. Cowan. 2004. Respirometry for Environmental Science and Engineering. SJ Enterprises. Springdale, Arkansas. 
Appendix A Physical and Biochemical Data 
Table A.1 Synthetic Wastewater

\begin{tabular}{|c|c|c|c|c|c|c|c|c|}
\hline \multirow{2}{*}{$\begin{array}{c}\text { Date } \\
\text { Sampled }\end{array}$} & \multicolumn{3}{|c|}{ COD (mg/L) } & \multicolumn{3}{c|}{ SS (mg/L) } & \multirow{2}{*}{$\mathrm{pH}$} \\
\hline & TCOD & SCOD & PCOD & ffCOD & TSS & VSS & ISS & \\
\hline 13 & 2508 & 1082 & 1426 & & & & & \\
19 & 2018 & & & & & & & 8.0 \\
19 & 2182 & & & & & & & 8.0 \\
34 & 7557 & & & & & & & \\
36 & 7201 & & & & 1320 & 1320 & 0 & 6.0 \\
41 & 4664 & & & & 1250 & 930 & 320 & \\
43 & 5225 & & & & & & & \\
48 & 5761 & & & & & & & 3.9 \\
69 & 7715 & & & & & & & \\
76 & 7806 & & & & & & & \\
83 & 7108 & & & & & & & \\
85 & 7432 & & & & & & & 4.6 \\
117 & & & & & & & & 6.2 \\
119 & 7379 & & & & & & & 4.5 \\
172 & & & & & & & \\
175 & 8754 & 6223 & 2531 & 5836 & 2210 & 1940 & 270 & 4.5 \\
181 & 4153 & 2918 & 1235 & 2718 & 1090 & 900 & 190 & 4.6 \\
193 & 3903 & 3080 & 823 & 2768 & 1020 & 860 & 160 & 4.6 \\
225 & 3654 & 2862 & 792 & 2619 & 810 & 750 & 60 & 4.5 \\
273 & 4103 & 2993 & 1110 & & & & & 4.5 \\
273 & 4028 & 2980 & 1047 & & 920 & 880 & 40 & 4.5 \\
273 & 4190 & 3030 & 1160 & & 880 & 820 & 60 & 4.5 \\
278 & 4015 & 3018 & 998 & 2899 & 880 & 790 & 90 & 4.5 \\
\hline$*$ Mean & 4006 & 2983 & 1023 & 2751 & 933 & 833 & 100 & 4.53 \\
$*$ Std. dev. & 183 & 73 & 166 & 117 & 103 & 57 & 61 & 0.05 \\
\hline
\end{tabular}

* Mean and standard deviation of data over Phases 1 and 2 from day 179 to 283 
Table A.2 BR WAS

\begin{tabular}{|c|c|c|c|c|c|c|c|c|c|c|}
\hline \multirow{2}{*}{$\begin{array}{l}\text { Date } \\
\text { Sampled }\end{array}$} & \multicolumn{4}{|c|}{$\mathrm{COD}(\mathrm{mg} / \mathrm{L})$} & \multicolumn{3}{|c|}{$\mathrm{SS}(\mathrm{mg} / \mathrm{L})$} & \multirow[t]{2}{*}{$\mathrm{pH}$} & \multicolumn{2}{|c|}{$\mathrm{N}(\mathrm{mg} / \mathrm{L})$} \\
\hline & TCOD & SCOD & PCOD & ffCOD & TSS & VSS & ISS & & $\mathrm{NO}_{3}$ & $\mathrm{NH}_{3}$ \\
\hline 11 & 3283 & 52 & 3230 & & 3780 & 3210 & 570 & & & \\
\hline 21 & 4150 & 54 & 4096 & & & & & 9.3 & & \\
\hline 27 & 4988 & 180 & 4808 & & & & & & & \\
\hline 34 & 9326 & 245 & 9081 & & 8350 & 6900 & 1450 & 9.4 & & \\
\hline 36 & 8218 & 200 & 8018 & & 7950 & 6540 & 1410 & 7.0 & & \\
\hline 40 & & & & & & & & 9.2 & 0.5 & \\
\hline 41 & 7328 & 176 & 7152 & & 7470 & 6050 & 1420 & 9.2 & 0.5 & \\
\hline 48 & 7258 & 121 & 7137 & & & & & 8.9 & & \\
\hline 63 & & & & & & & & 8.6 & 1.05 & \\
\hline 69 & 8068 & 145 & 7923 & 267 & & & & 8.1 & & \\
\hline 70 & 8611 & 202 & 8409 & & & & & 8.7 & & \\
\hline 76 & 10451 & 202 & 10249 & 160 & & & & 8.3 & 0.8 & \\
\hline 78 & 8829 & & & & & & & & & \\
\hline 83 & 11871 & 140 & 11732 & & & & & 8.5 & 0.5 & \\
\hline 85 & 8554 & & & & & & & 8.6 & & \\
\hline 90 & & & & & & & & 8.6 & 0.45 & \\
\hline 91 & 8554 & 142 & 8412 & & & & & 8.6 & & \\
\hline 92 & 8953 & 151 & 8803 & 113 & 8180 & 7120 & 1060 & 8.3 & & \\
\hline 103 & 7519 & 928 & 6592 & & 11630 & 5330 & 6300 & 6.5 & & \\
\hline 105 & 7108 & 922 & 6186 & & 6200 & 5580 & 620 & 7.8 & & \\
\hline 105 & 6603 & 955 & 5648 & & & & & 7.8 & & \\
\hline 112 & & & & & & & & 7.5 & 0.45 & 231 \\
\hline 113 & 4664 & 688 & 3975 & 708 & & & & 7.9 & & \\
\hline 116 & 8916 & 828 & 8088 & & & & & 6.4 & 0.85 & \\
\hline 118 & 9851 & 1465 & 8386 & & & & & 8.3 & & \\
\hline 120 & & & & & & & & 8.6 & 1 & 160 \\
\hline 124 & 9178 & 1665 & 7513 & 1634 & & & & 7.7 & & \\
\hline 124 & 8863 & 1771 & 7092 & 1702 & & & & 7.7 & & \\
\hline 127 & 9053 & 1334 & 7719 & & & & & 8.2 & 0.8 & 202 \\
\hline 132 & & & & & & & & 8.4 & 1.15 & 150 \\
\hline 133 & & & & & & & & 8.6 & 0.8 & \\
\hline 134 & 9203 & 1646 & 7557 & & & & & 8.0 & 0.6 & \\
\hline 159 & & & & & & & & 8.2 & 0.7 & \\
\hline 160 & 7806 & 1913 & 5893 & & & & & 4.8 & 0.9 & 294 \\
\hline 162 & 6989 & 280 & 6709 & & & & & 8.1 & 1.3 & 184 \\
\hline 163 & 8280 & & & & & & & 7.7 & & \\
\hline 168 & & & & & & & & 8.5 & 0.7 & \\
\hline 174 & & & & & & & & 7.9 & 0.6 & \\
\hline 175 & 9502 & 1877 & 7625 & 1833 & 7520 & 6640 & 880 & 7.6 & 0.5 & 236 \\
\hline 179 & & & & & & & & 8.3 & 0.4 & \\
\hline 180 & 8679 & 1113 & 7566 & 780 & 7305 & 6320 & 985 & 8.5 & 0.9 & \\
\hline
\end{tabular}


Table A.3 BR WAS

\begin{tabular}{|c|c|c|c|c|c|c|c|c|}
\hline \multirow{2}{*}{ Date Sampled } & \multicolumn{4}{|c|}{ COD $(\mathrm{mg} / \mathrm{L})$} & \multicolumn{3}{|c|}{ SS $(\mathrm{mg} / \mathrm{L})$} & \multirow{2}{*}{ TCOD/ } \\
\cline { 2 - 7 } & TCOD & SCOD & PCOD & ffCOD & TSS & VSS & ISS & VSS \\
\hline 193 & 4489 & 165 & 4325 & 47 & 3990 & 3330 & 660 & 1.35 \\
196 & 5761 & 165 & 5597 & 81 & 6590 & 2765 & 3825 & 2.08 \\
203 & 4053 & 90 & 3963 & 85 & 3840 & 3260 & 580 & 1.24 \\
208 & 4651 & 54 & 4598 & 59 & & & & \\
209 & 3878 & & & & 4150 & 3530 & 620 & 1.10 \\
215 & 3753 & 59 & 3695 & 46 & 3620 & 3140 & 480 & 1.20 \\
222 & 4078 & 35 & 4043 & 49 & 3820 & 3330 & 490 & 1.22 \\
223 & 4365 & 54 & 4311 & & & & & \\
228 & 4327 & 44 & 4283 & 44 & 3990 & 3430 & 560 & 1.26 \\
232 & & & & & & & & \\
233 & & & & & & & & \\
236 & 3741 & 34 & 3707 & 41 & & & 440 & \\
237 & & & & & 4030 & 3530 & 500 & 0.00 \\
243 & 3953 & 46 & 3907 & 47 & 3600 & 3140 & 460 & 1.26 \\
250 & 4040 & 49 & 3992 & 50 & 3420 & 3180 & 240 & 1.27 \\
258 & 3878 & 46 & 3832 & 59 & 3560 & 3130 & 430 & 1.24 \\
264 & 4626 & 56 & 4570 & 59 & 3960 & 3460 & 500 & 1.34 \\
271 & 4502 & 56 & 4446 & 51 & 3820 & 3400 & 420 & 1.32 \\
278 & 4539 & 51 & 4488 & 45 & 4210 & 3570 & 640 & 1.27 \\
283 & & & & & & & & \\
\hline$*$ Mean & 4170 & 52 & 4127 & 53 & 3835 & 3342 & 489 & 1.23 \\
$*$ Std. dev. & 324 & 14 & 334 & 12 & 246 & 167 & 103 & 0.08 \\
\hline
\end{tabular}

* Mean and standard deviation of data from day 193 to 283 when the BR was at steady-state 
Table A.4 BR WAS

\begin{tabular}{|c|c|c|c|c|c|c|}
\hline \multirow{2}{*}{$\begin{array}{c}\text { Date } \\
\text { Sampled }\end{array}$} & \multirow{2}{*}{$\mathrm{pH}$} & \multicolumn{4}{|c|}{$\mathrm{N}(\mathrm{mg} / \mathrm{L})$} & \multirow{2}{p}{$\begin{array}{c}\mathrm{pON} / \\
\mathrm{pCOD}\end{array}$} \\
\cline { 3 - 6 } 193 & 8.3 & 1.40 & 74 & & & \\
196 & 8.4 & 1.00 & 86 & & & \\
203 & 8.1 & 0.10 & 85 & & & \\
208 & 8.5 & 0.10 & 74 & & & \\
209 & 8.5 & & & & & \\
215 & 8.2 & 0.10 & 69 & & & \\
222 & 8.5 & 0.10 & 54 & & & \\
223 & 8.5 & & & & & \\
228 & 8.5 & 0.10 & 48 & & & \\
232 & 8.6 & & & 266 & 99 & \\
233 & 8.5 & 0.10 & 70 & & & \\
236 & 8.5 & 0.10 & 76 & 258 & 85 & 0.046 \\
237 & 8.3 & & & & & \\
243 & 8.0 & 0.10 & 78 & 277 & 79 & 0.051 \\
250 & 8.6 & 0.10 & 79 & 301 & 87 & 0.053 \\
258 & 8.4 & 0.20 & 74 & 285 & 81 & 0.053 \\
264 & 8.6 & 0.20 & 73 & 284 & 78 & 0.045 \\
271 & 8.4 & 0.10 & 84 & 348 & 90 & 0.058 \\
278 & 8.3 & 0.40 & 80 & 358 & 80 & 0.062 \\
283 & 8.5 & & & & & \\
\hline$*$ Mean & 8.52 & 0.14 & 73 & 297 & 85 & 0.053 \\
$*$ Std. dev. & 0.12 & 0.09 & 11 & 36.9 & 7 & 0.006 \\
\hline
\end{tabular}

*Mean and standard deviation of data from day 193 to 283 when the BR was at steady-state 
Table A.5 Pretreated BR WAS

\begin{tabular}{|c|c|c|c|c|c|c|c|c|}
\hline \multirow{2}{*}{$\begin{array}{c}\text { Date } \\
\text { Sampled }\end{array}$} & \multicolumn{4}{|c|}{ COD $(\mathrm{mg} / \mathrm{L})$} & \multicolumn{3}{|c|}{ SS (mg/L) } & TCOD/ \\
\cline { 2 - 7 } & TCOD & SCOD & PCOD & ffCOD & TSS & VSS & ISS & VSS \\
\hline 193 & 4228 & 2470 & 1758 & 1853 & 2098 & 1657 & 441 & 2.55 \\
196 & 4305 & 2080 & 2225 & 1560 & 1917 & 1514 & 403 & 2.84 \\
197 & 4285 & 2005 & 2280 & 1504 & 2100 & 1659 & 441 & 2.58 \\
200 & 4105 & 2503 & 1602 & 1877 & & & & \\
201 & 4366 & 2494 & 1872 & 1871 & & & & \\
202 & 4315 & 2510 & 1805 & 1883 & & & & \\
203 & 4100 & 2320 & 1780 & 1740 & 2320 & 1833 & 487 & 2.24 \\
209 & 4215 & 2606 & 1609 & 1846 & 2090 & 1590 & 500 & 2.65 \\
215 & 4165 & 2506 & 1659 & 1746 & 1970 & 1440 & 530 & 2.89 \\
222 & 4153 & 2669 & 1484 & 1802 & 1660 & 1270 & 390 & 3.27 \\
223 & 4290 & 2494 & 1796 & & & & & \\
228 & 4402 & 2694 & 1708 & 2001 & 1740 & 1240 & 500 & 3.55 \\
236 & 4090 & 2475 & 1615 & 1908 & & & & \\
237 & & & & & 1840 & 1440 & 400 & \\
243 & 3778 & 2213 & 1565 & 1715 & 1720 & 1280 & 440 & 2.95 \\
250 & 3965 & 2213 & 1752 & 1546 & 1830 & 1780 & 50 & 2.23 \\
254 & & & & & 1610 & 1210 & 400 & 0.00 \\
258 & 3741 & 2176 & 1565 & 1727 & 2280 & 1730 & 550 & 2.16 \\
264 & 4227 & 2226 & 2001 & 1659 & 2200 & 1710 & 490 & 2.47 \\
271 & 4365 & 2076 & 2288 & 1652 & 2020 & 1710 & 310 & 2.55 \\
278 & 4315 & 2082 & 2232 & 1378 & 2270 & 1810 & 460 & 2.38 \\
283 & & & & & & & & \\
$*$ Mean & 4142 & 2369 & 1773 & 1725 & 1936 & 1518 & 412 & 2.73 \\
$*$ Std. dev. & 215 & 228 & 264 & 171 & 237 & 230 & 131 & 0.79 \\
\hline
\end{tabular}

*Mean and standard deviation of data from day 193 to 283 when the BR was at steady-state 
Table A.6 Pretreated BR WAS

\begin{tabular}{|c|c|c|c|c|c|c|}
\hline \multirow{2}{*}{$\begin{array}{c}\text { Date } \\
\text { Sampled }\end{array}$} & \multirow{2}{*}{$\mathrm{pH}$} & \multicolumn{4}{|c|}{$\mathrm{N}(\mathrm{mg} / \mathrm{L})$} & \multirow{2}{*}{$\begin{array}{c}\mathrm{pON} / \\
\mathrm{pCOD}\end{array}$} \\
\hline & & $\mathrm{NO}_{3}$ & $\mathrm{NH}_{3}$ & $\mathrm{TKN}$ & $\mathrm{sTKN}$ & $\mathrm{n}$ \\
\hline 193 & 7.3 & 2.1 & & & & \\
196 & 6.1 & 2.2 & 108 & & & \\
197 & 7.5 & 3.8 & 88 & & & \\
200 & 7.2 & 4.5 & 97 & & & \\
201 & 7.8 & 1.2 & 75 & & & \\
202 & 7.8 & 5 & 79 & & & \\
203 & 7.6 & 3.0 & 81 & & & \\
209 & 7.9 & 2.1 & 84 & & & \\
215 & 7.7 & 1.8 & 83 & & & \\
222 & 8.1 & 1.8 & 59 & & & \\
223 & 7.9 & & & & & \\
228 & 8.1 & 2.4 & 62 & & & \\
232 & 7.9 & & & 309 & 231 & \\
233 & 7.8 & 2.1 & 81 & & & \\
236 & 7.6 & 2.5 & 95 & 281 & 198 & 0.051 \\
237 & 7.7 & & & & & \\
243 & 8.0 & 2.2 & 92 & 262 & 184 & 0.050 \\
250 & 7.9 & 3.1 & 92 & 276 & 200 & 0.043 \\
254 & 8.2 & & & & & \\
258 & 8.0 & 4.1 & 87 & 314 & 205 & 0.069 \\
264 & 8.0 & 3.9 & 96 & 286 & 193 & 0.047 \\
271 & 8.0 & 3.9 & 91 & 306 & 196 & 0.048 \\
278 & 7.7 & 3.9 & 92 & 317 & 210 & 0.048 \\
283 & 7.5 & & & & & \\
\hline *Mean & 7.8 & 2.9 & 84 & 294 & 202 & 0.052 \\
$*$ Std. dev. & 0.2 & 1.1 & 12 & 20 & 14 & 0.016 \\
\hline & & & & & & \\
\hline
\end{tabular}

*Mean and standard deviation of data from day 193 to 283 when the BR was at steady-state 
Table A.7 AD WAS

\begin{tabular}{|c|c|c|c|c|c|c|c|c|c|c|}
\hline \multirow{2}{*}{$\begin{array}{l}\text { Date } \\
\text { Sampled }\end{array}$} & \multicolumn{4}{|c|}{$\mathrm{COD}(\mathrm{mg} / \mathrm{L})$} & \multicolumn{3}{|c|}{$\mathrm{SS}(\mathrm{mg} / \mathrm{L})$} & \multirow{2}{*}{$\mathrm{pH}$} & \multicolumn{2}{|c|}{$\mathrm{N}(\mathrm{mg} / \mathrm{L})$} \\
\hline & TCOD & SCOD & PCOD & ffCOD & TSS & VSS & ISS & & $\mathrm{NO}_{3}$ & $\mathrm{NH}_{3}$ \\
\hline 27 & 6709 & 185 & 6524 & & & & & & & \\
\hline 34 & 11809 & 429 & 11380 & & 11880 & 9140 & 2740 & 9.3 & & \\
\hline 36 & 11491 & 370 & 11121 & & 12590 & 9540 & 3050 & 9.3 & & \\
\hline 41 & 13037 & 379 & 12658 & & 15000 & 10950 & 4050 & 9.3 & 1.5 & \\
\hline 63 & & & & & & & & 8.4 & 3.9 & \\
\hline 69 & 16909 & & & & & & & 8.1 & & \\
\hline 70 & 16305 & 409 & 15896 & & & & & 8.0 & & \\
\hline 78 & 14590 & 625 & & 352 & & & & & 415 & \\
\hline 83 & 10924 & 549 & 13965 & & & & & 6.7 & 340 & \\
\hline 90 & & & 10375 & & & & & 6.3 & 335 & \\
\hline 91 & 9527 & 481 & & & & & & 6.1 & & \\
\hline 92 & 8380 & 384 & 9046 & 237 & 8030 & 6630 & 1400 & 6.8 & 290 & \\
\hline 95 & & & 7996 & & & & & 7.9 & 198 & \\
\hline 103 & 6385 & 269 & & & 6270 & 4970 & 1300 & 8.1 & 56 & 420 \\
\hline 104 & & & 6115 & & & & & 8.1 & & 348 \\
\hline 105 & 5836 & 248 & & & 5580 & 4530 & 1050 & 8.3 & & \\
\hline 112 & & & 5588 & & & & & 8.3 & 12 & 213 \\
\hline 116 & 3965 & 251 & & & & & & 8.3 & 34 & \\
\hline 118 & 5237 & 364 & 3715 & & & & & 8.0 & & \\
\hline 120 & & & 4873 & & & & & 6.5 & 305 & 29 \\
\hline 124 & 6609 & 451 & & 408 & & & & 6.9 & & \\
\hline 127 & 6011 & 414 & 6158 & & & & & 7.8 & 340 & 1 \\
\hline 132 & & & 5597 & & & & & 8.4 & 160 & 0 \\
\hline 134 & 5138 & 448 & & & & & & 8.6 & 92 & \\
\hline 159 & & & 4690 & & & & & 8.7 & 0.9 & \\
\hline 160 & 4053 & 434 & & & & & & 8.7 & 0.8 & 173 \\
\hline 162 & & & 3619 & & & & & 8.6 & 0.7 & 176 \\
\hline 163 & 3816 & 424 & & & & & & 8.5 & 0.6 & 159 \\
\hline 168 & & & 3392 & & & & & 8.5 & 0.8 & \\
\hline 174 & & & & & & & & 8.3 & 50 & \\
\hline 175 & 4788 & 426 & & 342 & 5240 & 3550 & 1690 & 8.4 & 36 & 150 \\
\hline 179 & & & 4362 & & & & & 8.5 & 8.5 & \\
\hline 181 & 4639 & 416 & & 267 & 5120 & 3460 & 1660 & 8.3 & 3.4 & \\
\hline 186 & & & & & & & & 8.4 & 2.2 & \\
\hline 193 & 2868 & 463 & 2405 & 291 & 3190 & 1990 & 1200 & 8.4 & 0.7 & 136 \\
\hline
\end{tabular}


Table A.8 AD WAS Phase 1

\begin{tabular}{|c|c|c|c|c|c|c|c|c|c|c|c|}
\hline \multirow{2}{*}{$\begin{array}{l}\text { Date } \\
\text { Sampled }\end{array}$} & \multicolumn{4}{|c|}{$\mathrm{COD}(\mathrm{mg} / \mathrm{L})$} & \multicolumn{3}{|c|}{$\mathrm{SS}(\mathrm{mg} / \mathrm{L})$} & \multirow{2}{*}{$\begin{array}{l}\text { TCOD } \\
\text { / VSS }\end{array}$} & \multirow{2}{*}{$\mathrm{pH}$} & \multicolumn{2}{|c|}{$\mathrm{N}(\mathrm{mg} / \mathrm{L})$} \\
\hline & TCOD & SCOD & PCOD & ffCOD & TSS & VSS & ISS & & & $\mathrm{NO}_{3}$ & $\mathrm{NH}_{3}$ \\
\hline 197 & 2207 & 340 & 1.36 & 203 & 2510 & 1620 & 890 & 1.36 & 8.5 & 1.0 & 122 \\
\hline 201 & 2556 & 304 & 1.62 & 183 & 2570 & 1580 & 990 & 1.62 & 8.4 & 0.7 & 106 \\
\hline 203 & 2519 & 282 & 1.40 & 185 & 2860 & 1800 & 1060 & 1.40 & 8.3 & 0.6 & 107 \\
\hline *Mean & 2427 & 309 & 1.46 & 190 & 2647 & 1667 & 980 & 1.46 & 8.40 & 0.8 & 112 \\
\hline$*$ Std dev & 192 & 30 & 0.14 & 11 & 187 & 117 & 85 & 0.14 & 0.10 & 0.2 & 9 \\
\hline
\end{tabular}

*Mean and standard deviation of data from day 197 to 203 when the AD was at steady-state during Phase 1

Table A.9 AD WAS Phase 2

\begin{tabular}{|l|l|l|l|l|l|l|l|l|}
\hline \multirow{2}{*}{$\begin{array}{l}\text { Date } \\
\text { Sampled }\end{array}$} & \multicolumn{3}{|l|}{ COD $(\mathrm{mg} / \mathrm{L})$} & \multicolumn{3}{l|}{ SS $(\mathrm{mg} / \mathrm{L})$} & \multirow{2}{*}{ TCOD } \\
\cline { 2 - 7 } & TCOD & SCOD & PCOD & ffCOD & TSS & VSS & ISS & /VSS \\
\hline 208 & 2234 & 269 & 1965 & 165 & & & & \\
209 & 2120 & 258 & 1862 & 167 & 2190 & 1410 & 780 & 1.50 \\
222 & 2132 & 301 & 1832 & 190 & 2100 & 1390 & 710 & 1.53 \\
228 & 1833 & 337 & 1496 & 207 & 1700 & 1270 & 430 & 1.44 \\
236 & 2051 & 315 & 1736 & 213 & 1780 & 1250 & 530 & 1.64 \\
237 & 2369 & 283 & 2086 & 208 & & & 440 & \\
243 & & & & & 2070 & 1560 & 510 & \\
250 & 2114 & 271 & 1843 & 197 & 2010 & 1430 & 580 & 1.48 \\
258 & 2164 & 239 & 1924 & 188 & 2160 & 1640 & 520 & 1.32 \\
264 & 2014 & 241 & 1873 & 201 & 2120 & 1633 & 487 & 1.29 \\
271 & 2058 & 205 & 1745 & 200 & 1950 & 1480 & 470 & 1.36 \\
278 & 2126 & 197 & 1929 & 151 & 2070 & 1530 & 540 & 1.34 \\
283 & & & & & 2200 & 1630 & 570 & 1.30 \\
\hline$*$ Mean & 2098 & 237 & 1861 & 177 & 2085 & 1557 & 528 & 1.39 \\
$*$ Std. dev. & 53 & 31 & 67 & 31 & 94 & 90 & 44 & 0.08 \\
\hline
\end{tabular}

*Mean and standard deviation of data from day 243 to 283 when the AD was at steady-state during Phase 2 
Table A.10 AD WAS Phase 2

\begin{tabular}{|l|l|l|l|l|l|l|}
\hline \multirow{2}{*}{$\begin{array}{l}\text { Date } \\
\text { Sampled }\end{array}$} & & \multicolumn{3}{|l|}{$\mathrm{N}(\mathrm{mg} / \mathrm{L})$} & \multirow{2}{*}{$\begin{array}{l}\text { pON/ } \\
\text { pCOD }\end{array}$} \\
\cline { 3 - 6 } 208 & 8.4 & 0.6 & 103 & & & \\
209 & 8.3 & 0.6 & 104 & & & \\
214 & 8.4 & 1.5 & & & & \\
215 & 8.2 & 1.6 & 99 & & & \\
222 & 8.5 & 1.1 & 87 & & & \\
228 & 8.4 & 1.2 & 62 & & & \\
232 & 8.1 & & & 273 & 188 & \\
233 & 8.4 & 1.2 & 86 & & & \\
236 & 8.4 & 1.1 & 97 & 231 & 108 & 0.059 \\
237 & 8.3 & & & & & \\
243 & 8.1 & 0.9 & 90 & 222 & 97 & 0.068 \\
250 & 8.4 & 0.8 & 89 & 263 & 108 & 0.081 \\
258 & 8.2 & 0.9 & 90 & 253 & 104 & 0.079 \\
264 & 8.2 & 1.0 & 113 & 251 & 121 & 0.075 \\
271 & 8.2 & 0.7 & 103 & 297 & 119 & 0.096 \\
278 & 8.1 & 1.0 & 100 & 299 & 112 & 0.097 \\
283 & 8.0 & & & & & \\
\hline *Mean & 8.32 & 0.9 & 98 & 264 & 110 & 0.075 \\
$*$ Std. dev. & 0.16 & 0.1 & 10 & 29 & 9 & 0.012 \\
\hline
\end{tabular}

*Mean and standard deviation of data from day 243 to 283 when the AD was at steady-state during Phase 2 
Appendix B Online Respirometry Data 


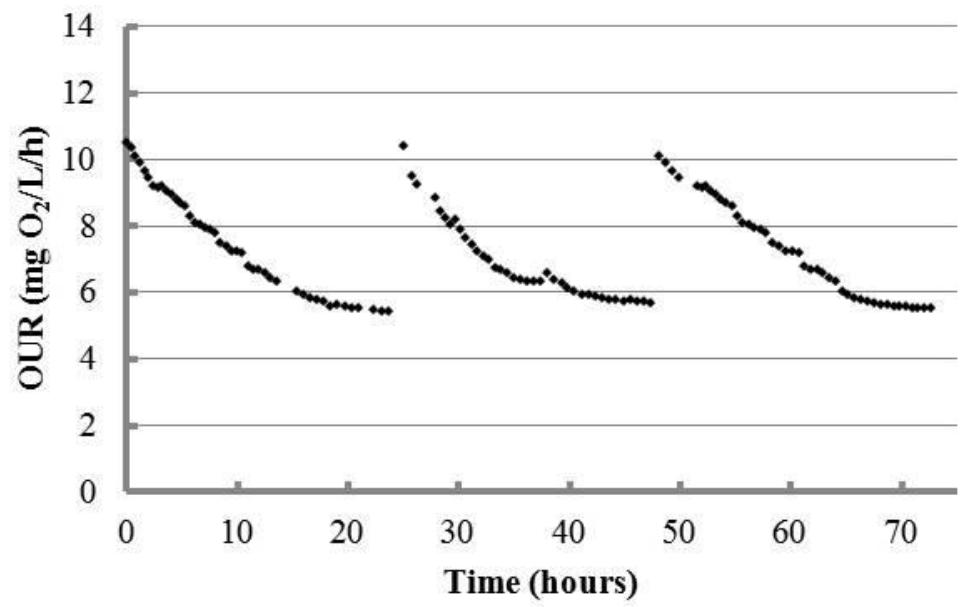

Figure B.1 Phase 2 Day 197 to 199

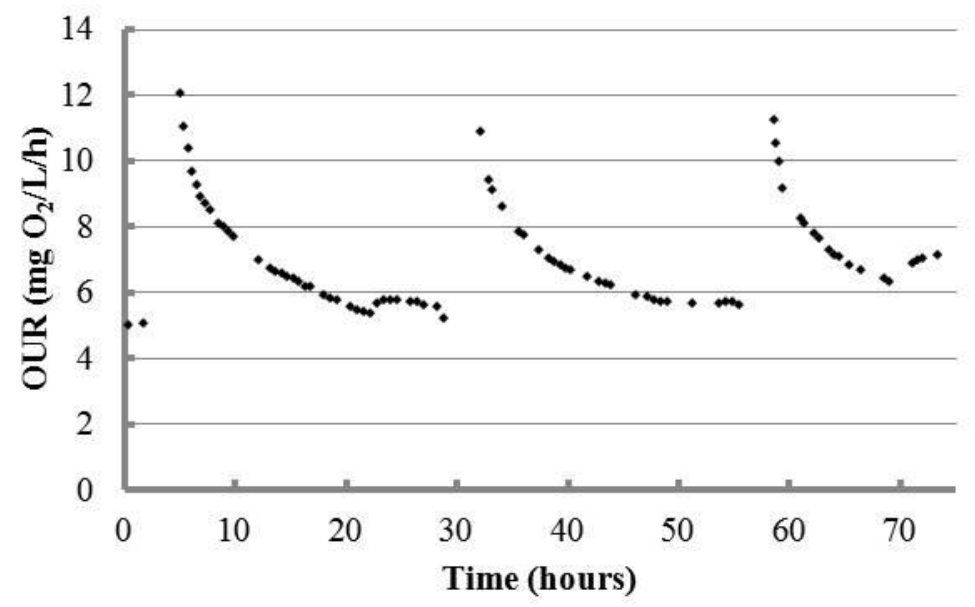

Figure B.2 Phase 2 Day 206 to 208

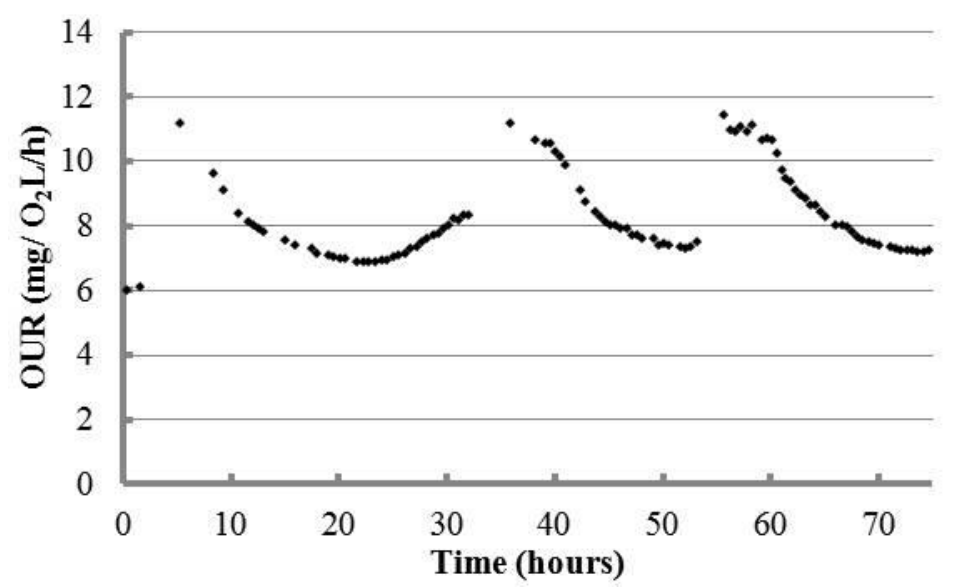

Figure B.3 Phase 2 Day 212 to 214 


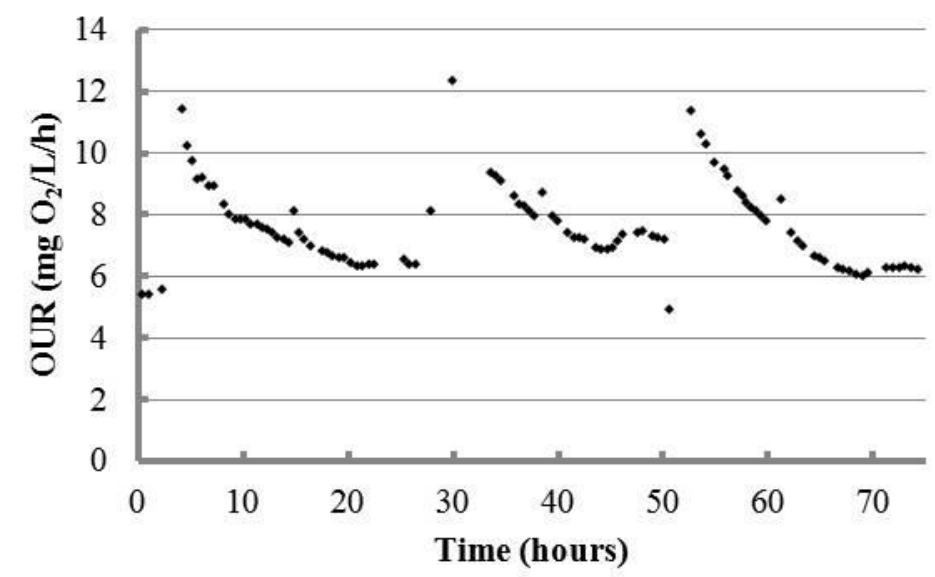

Figure B.4 Phase 2 Day 228 to 230

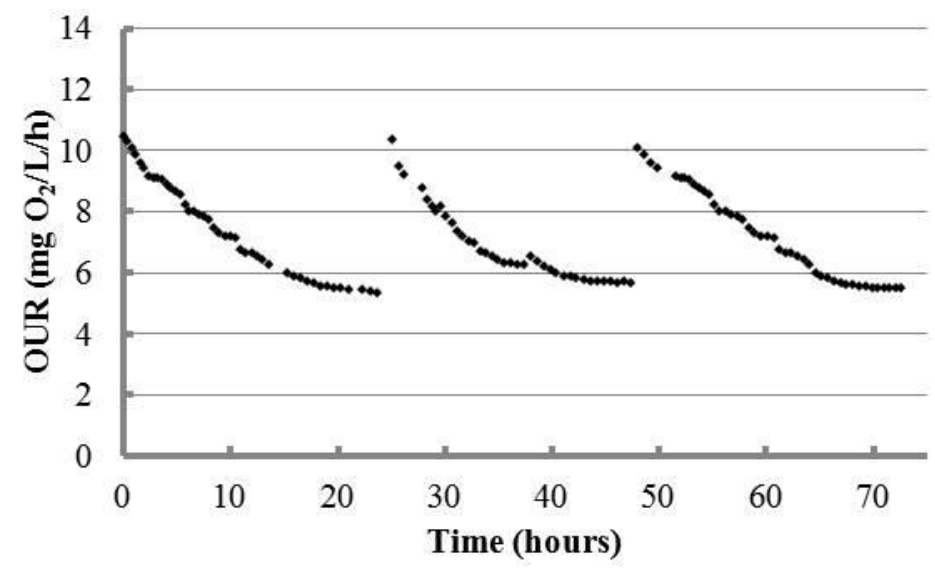

Figure B.5 Phase 2 Day 234 to 236

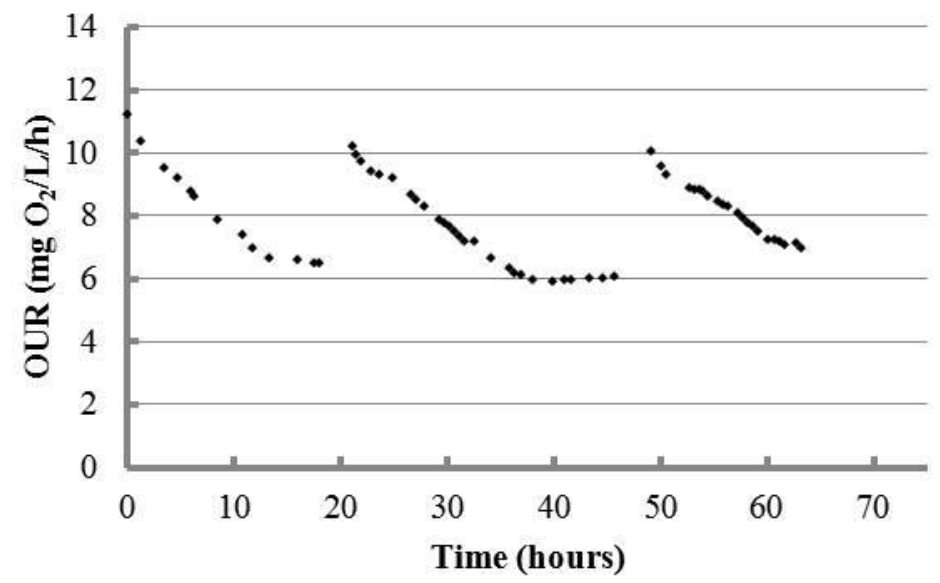

Figure B.6 Phase 2 Day 240 to 242 


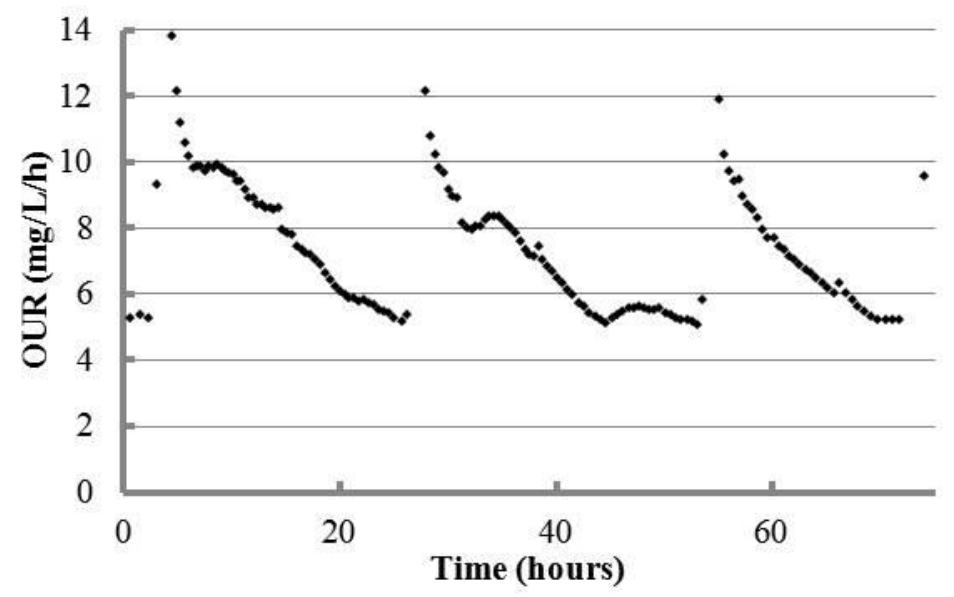

Figure B.7 Phase 2 Day 246 to 248

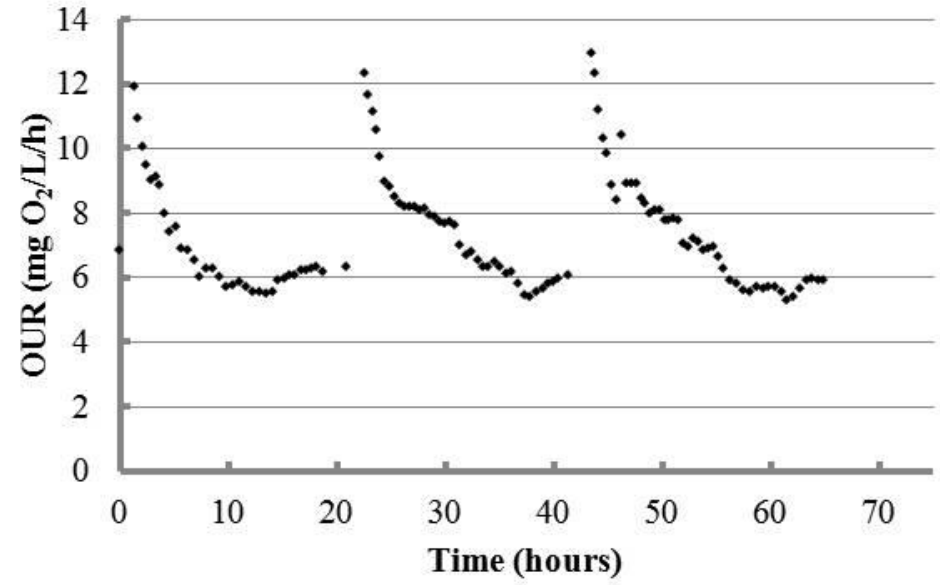

Figure B.8 Phase 2 Day 252 to 254

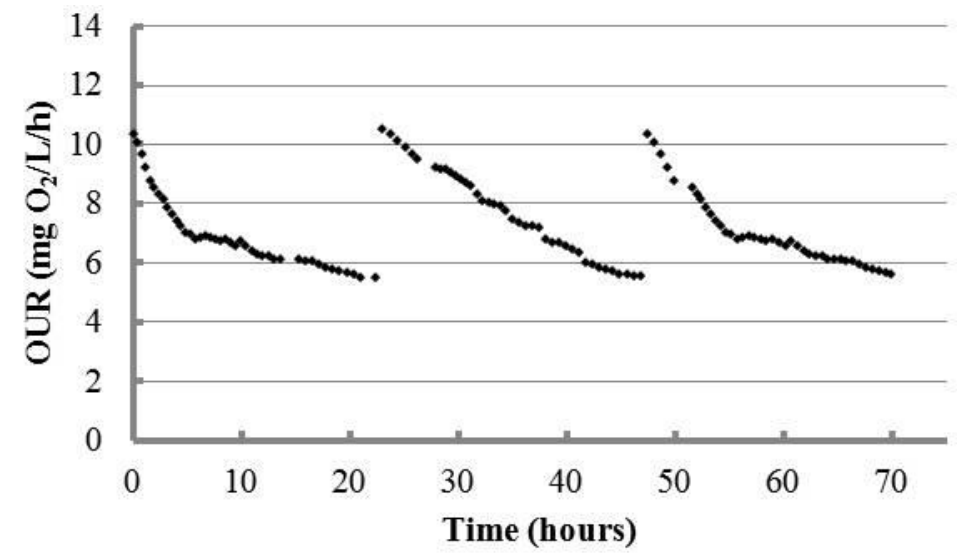

Figure B.9 Phase 2 Day 258 to 260 


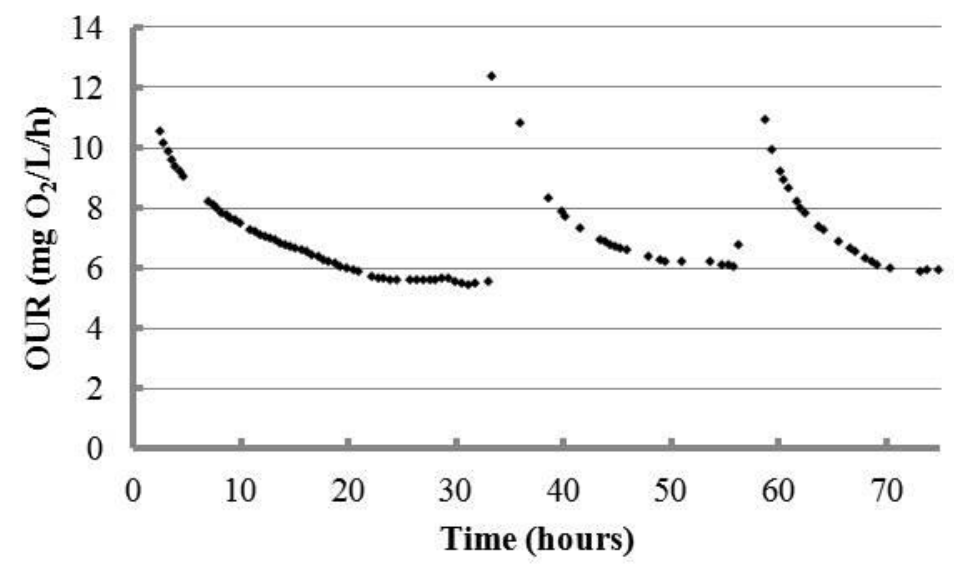

Figure B.10 Phase 2 Day 264 to 266

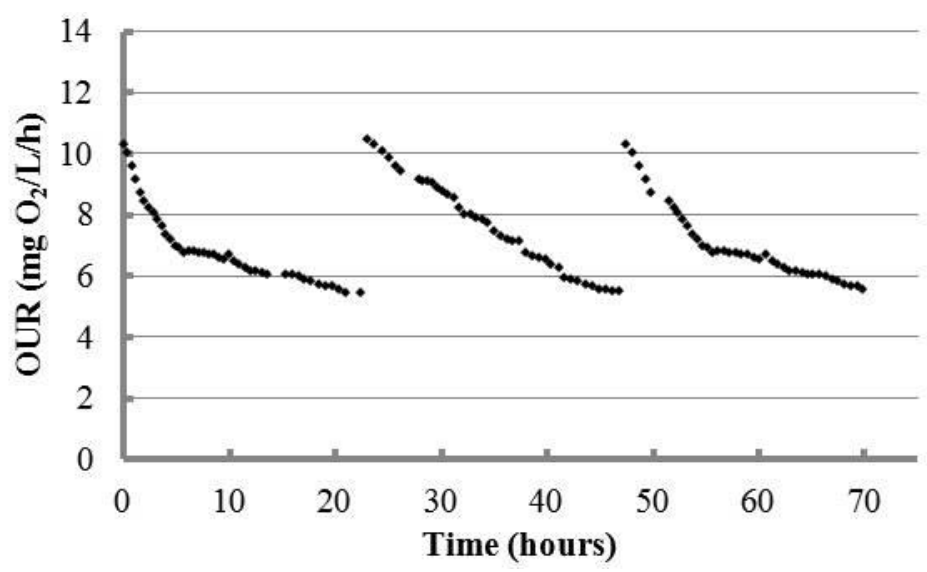

Figure B.11 Phase 2 Day 270 to 272

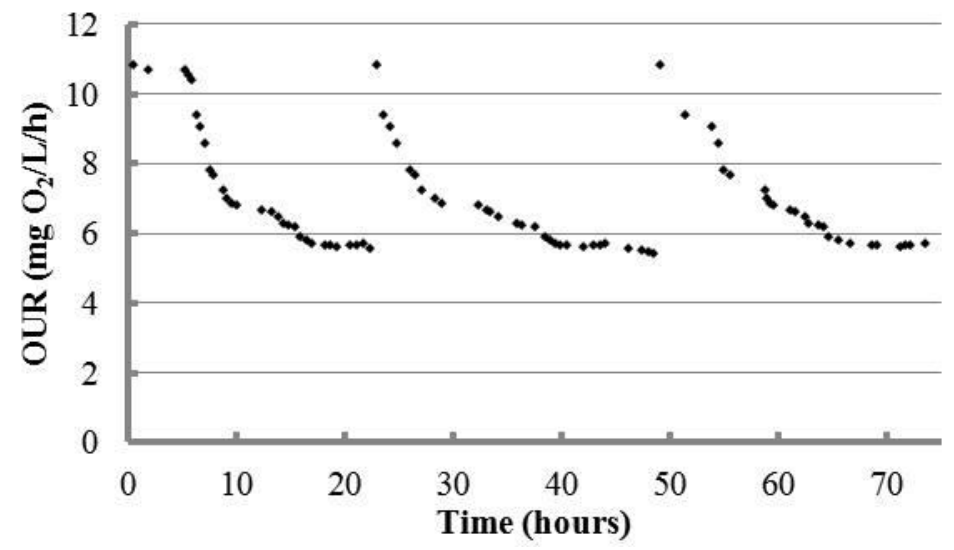

Figure B.12 Phase 2 Day 275 to 277 


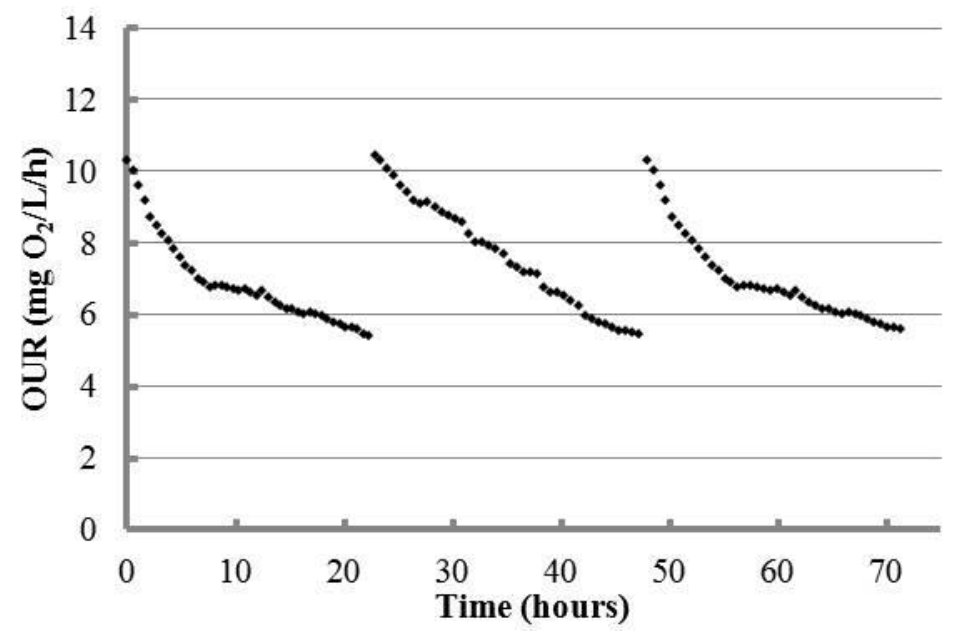

Figure B.13 Phase 2 Day 280 to 282

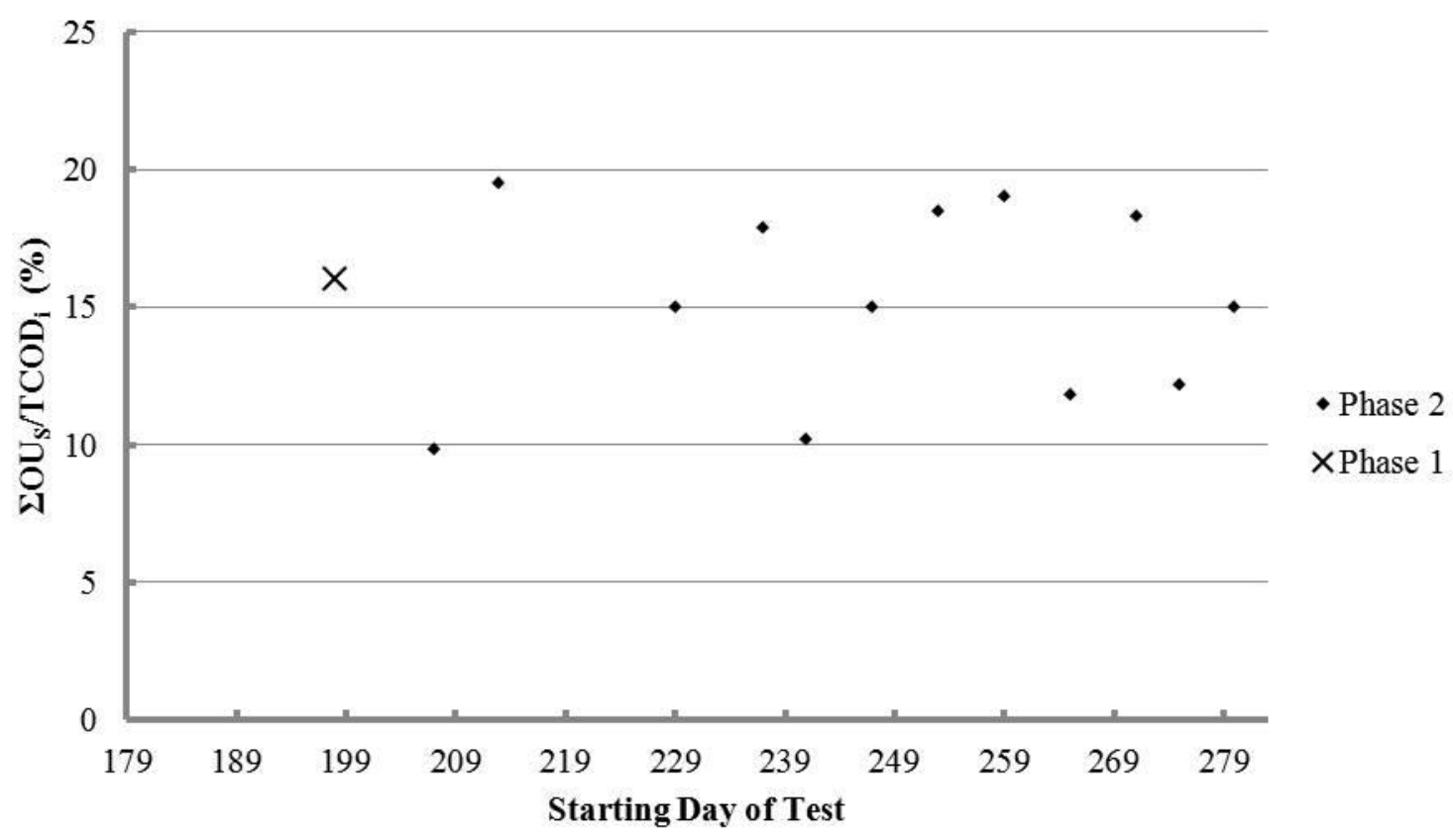

Figure B.14 Measured $\Sigma O U_{s} / T_{C O D}$ Ratios of BR WAS Fed to AD 
Appendix C Offline Respirometry Data 


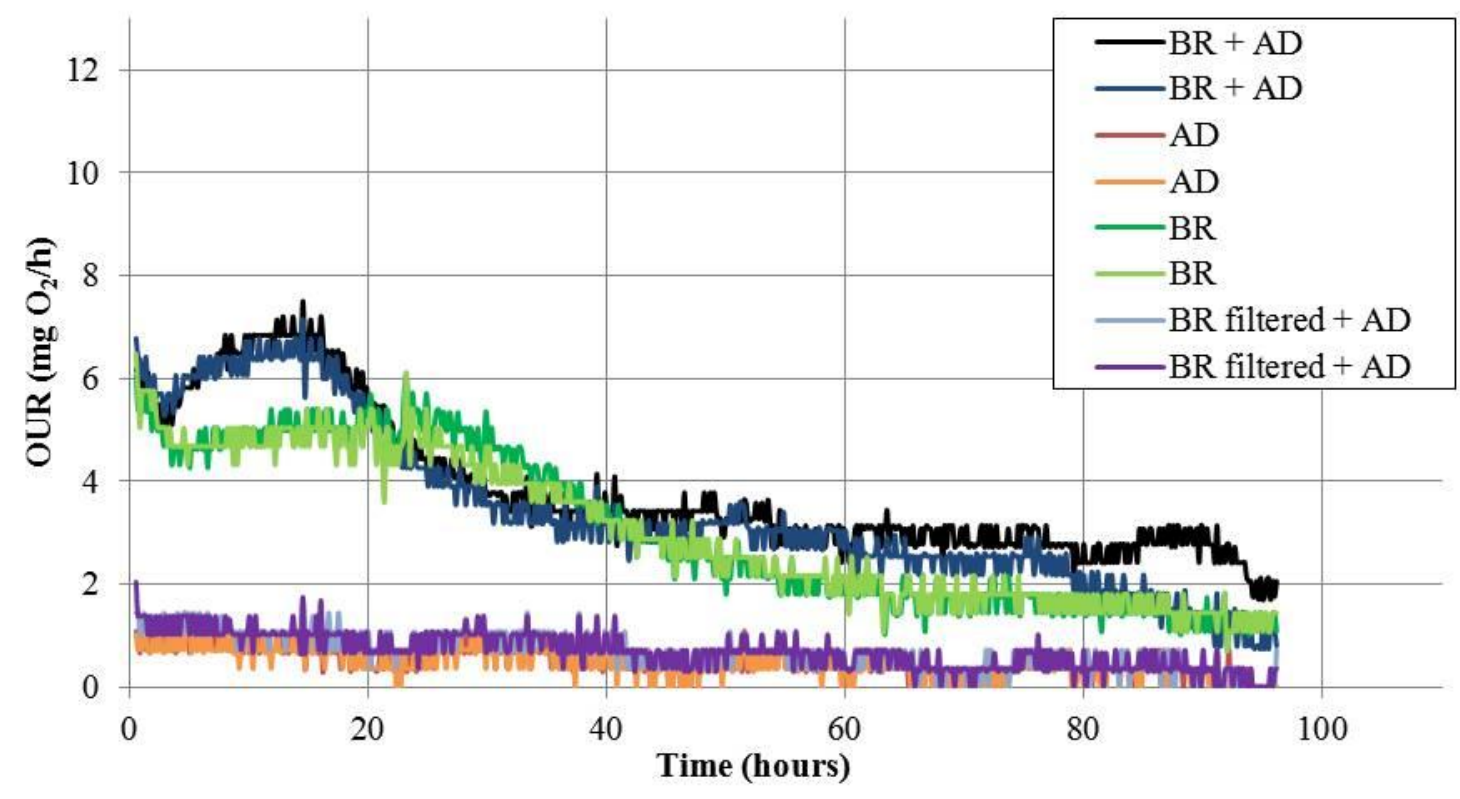

Figure C.1 BR WAS and AD WAS - Test Started on Day 193

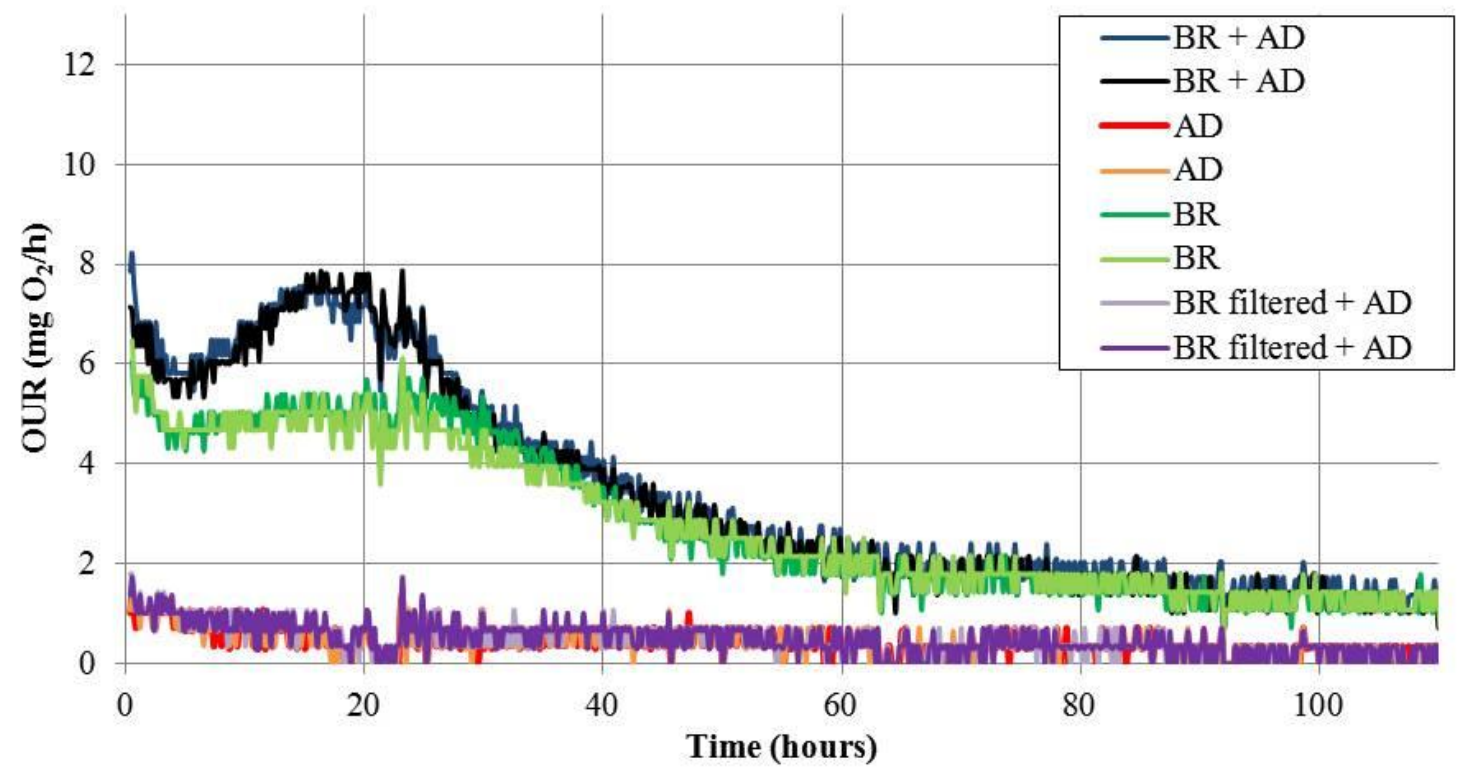

Figure C.2 BR WAS and AD WAS - Test Started on Day 203 


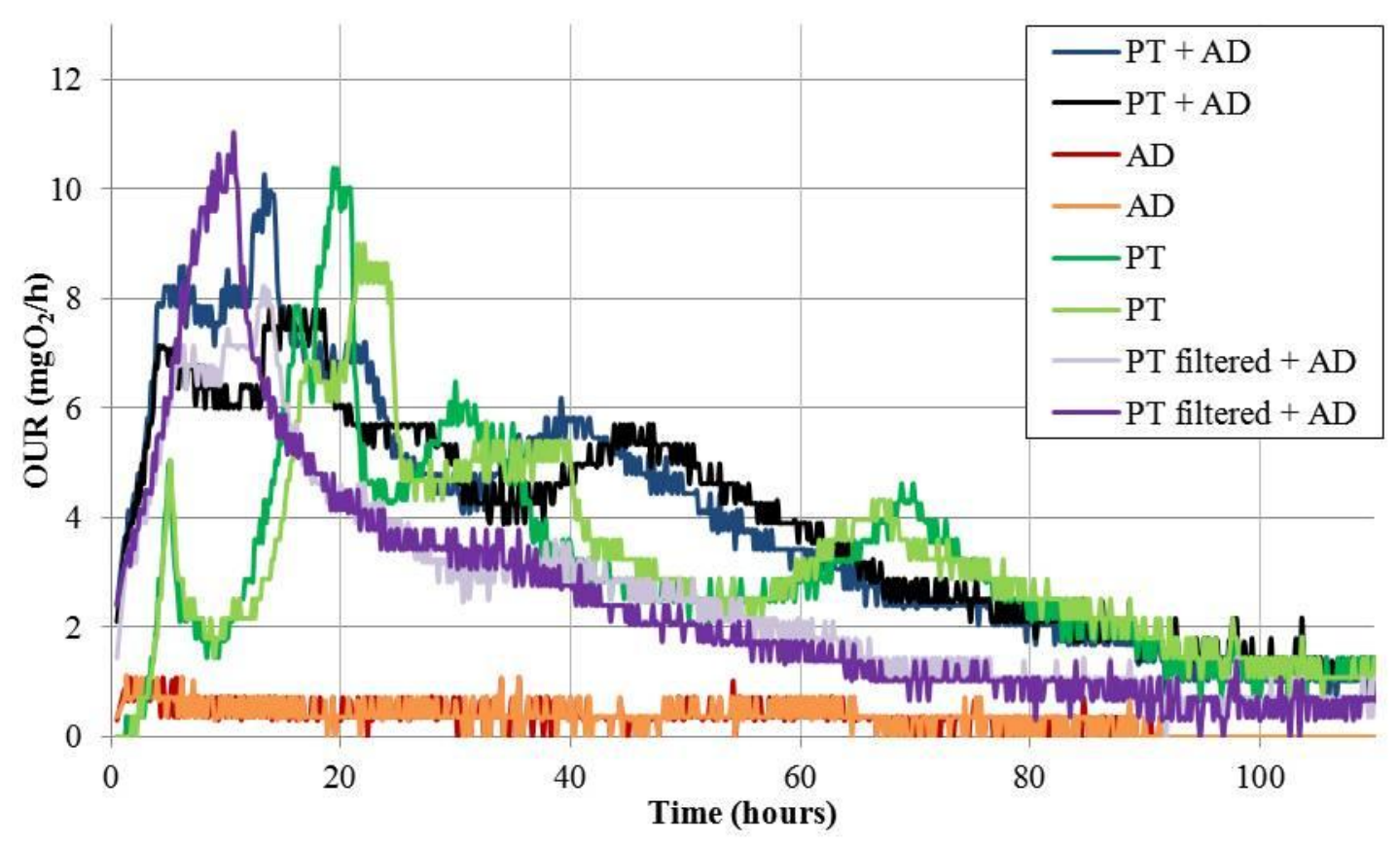

Figure C.3 Pretreated BR WAS and Non-acclimatized AD WAS - Test Started on Day 197

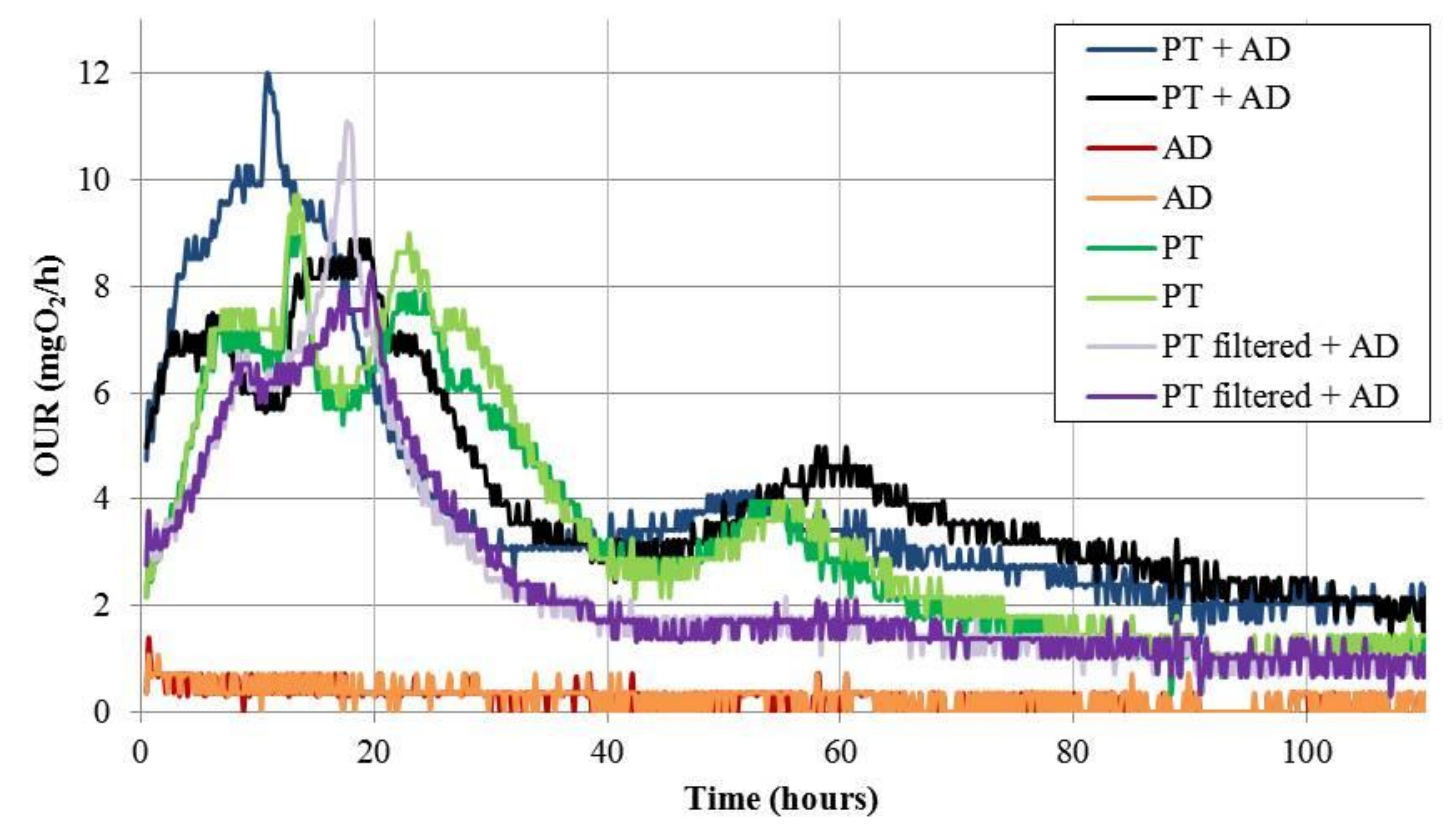

Figure C.4 Pretreated BR WAS and Acclimatized AD WAS - Test Started on Day 215 


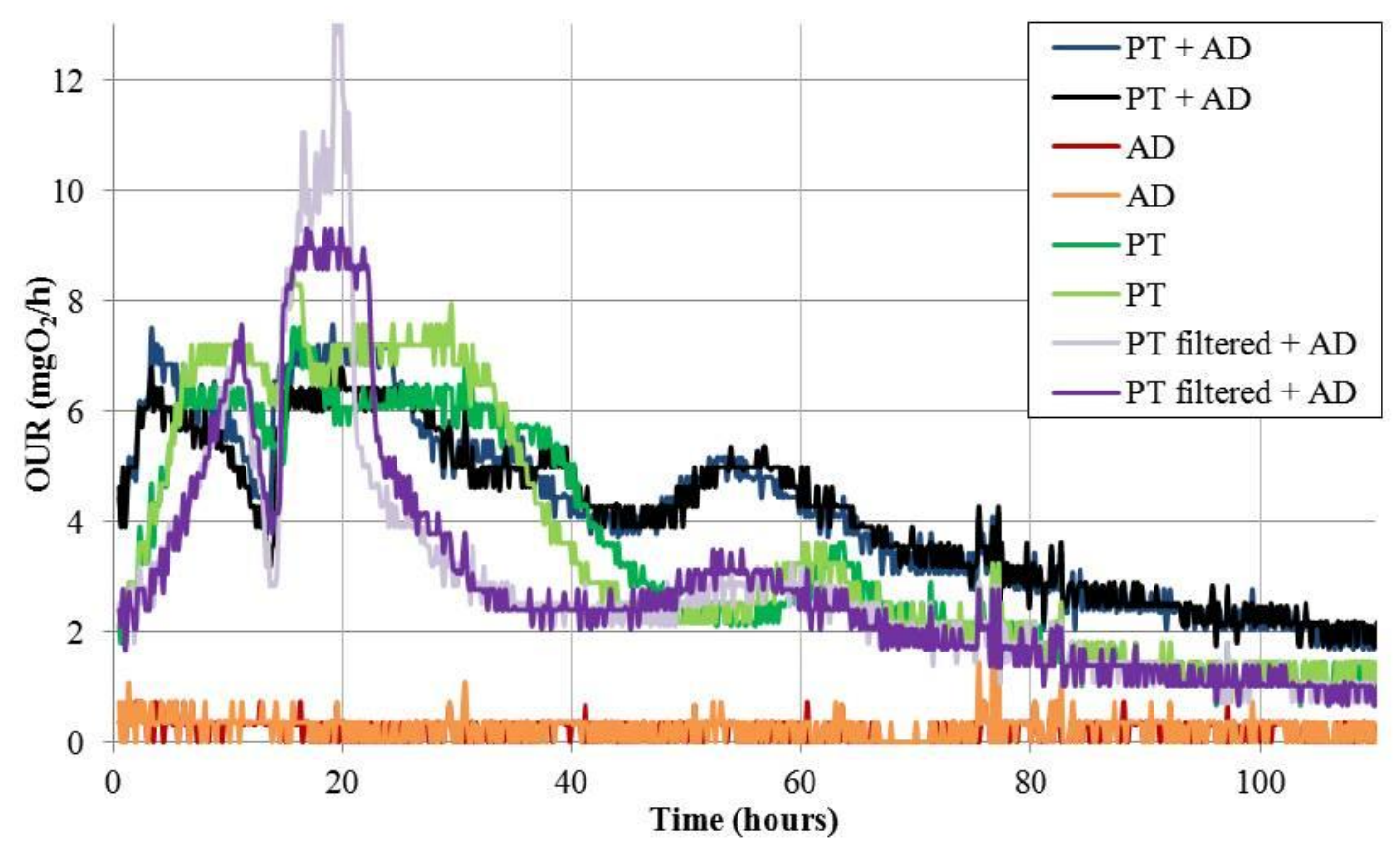

Figure C.5 Pretreated BR WAS and Acclimatized AD WAS - Test Started on Day 222

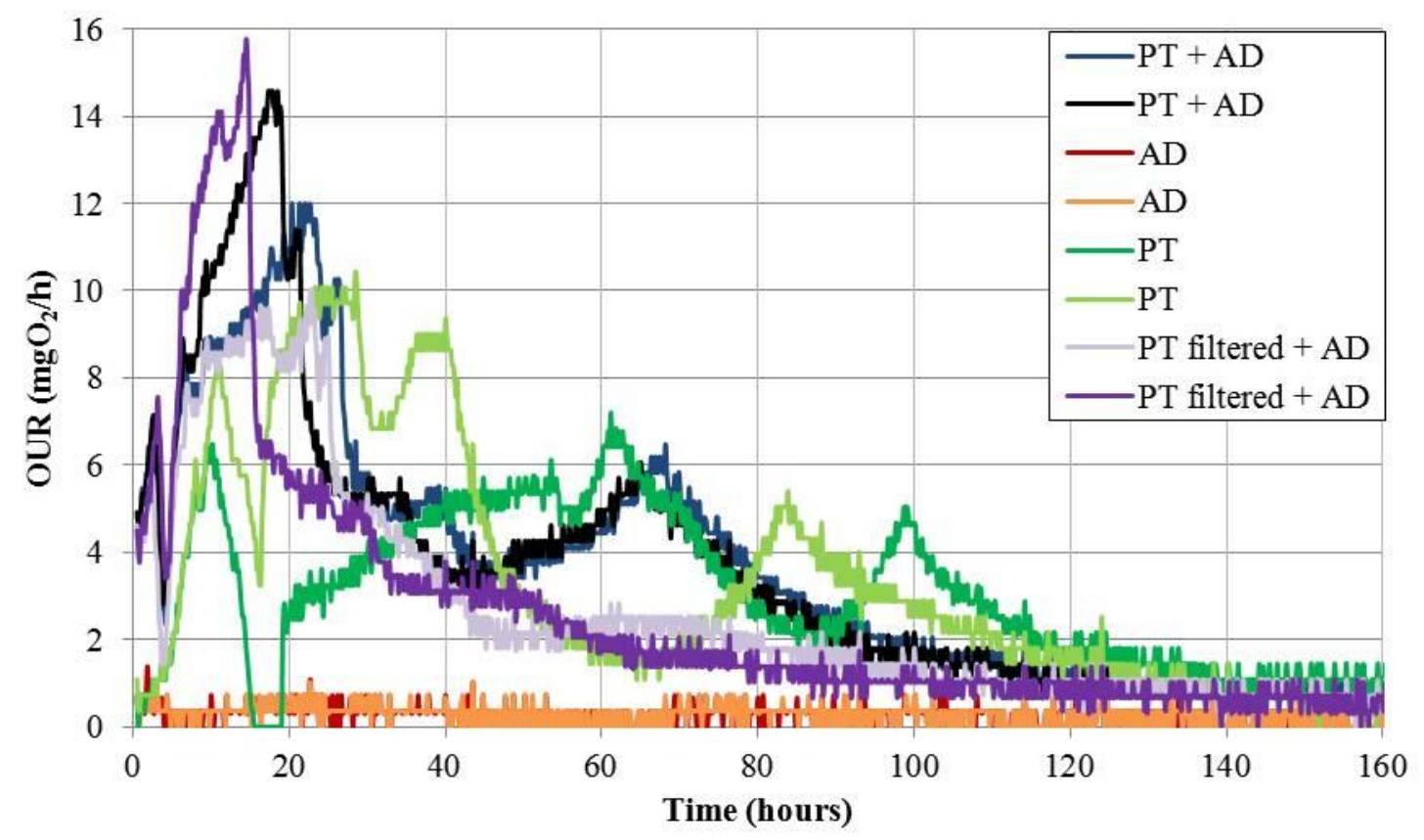

Figure C.6 Pretreated BR WAS and Acclimatized AD WAS - Test Started on Day 228 


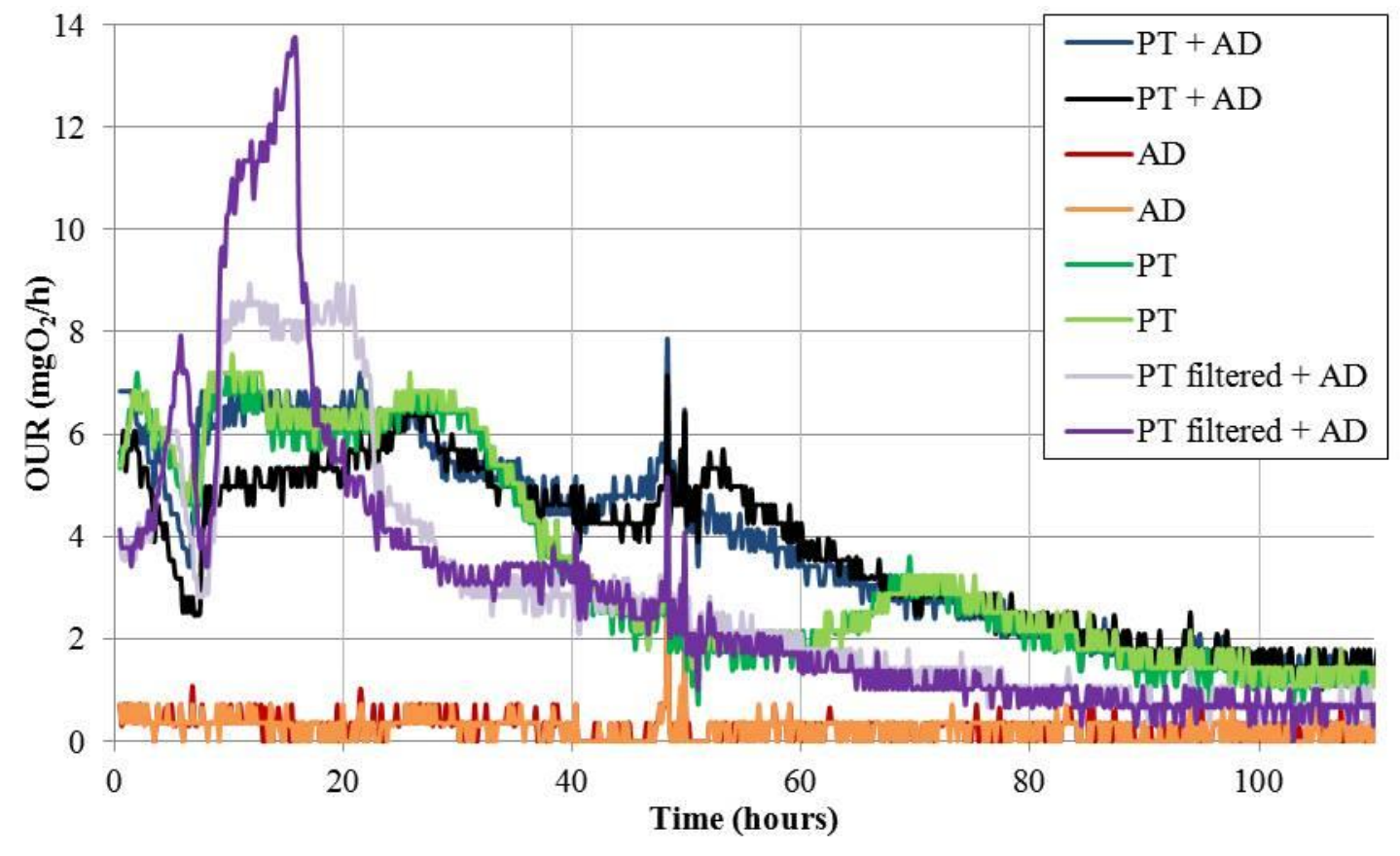

Figure C.7 Pretreated BR WAS and Acclimatized AD WAS - Test Started on Day 236

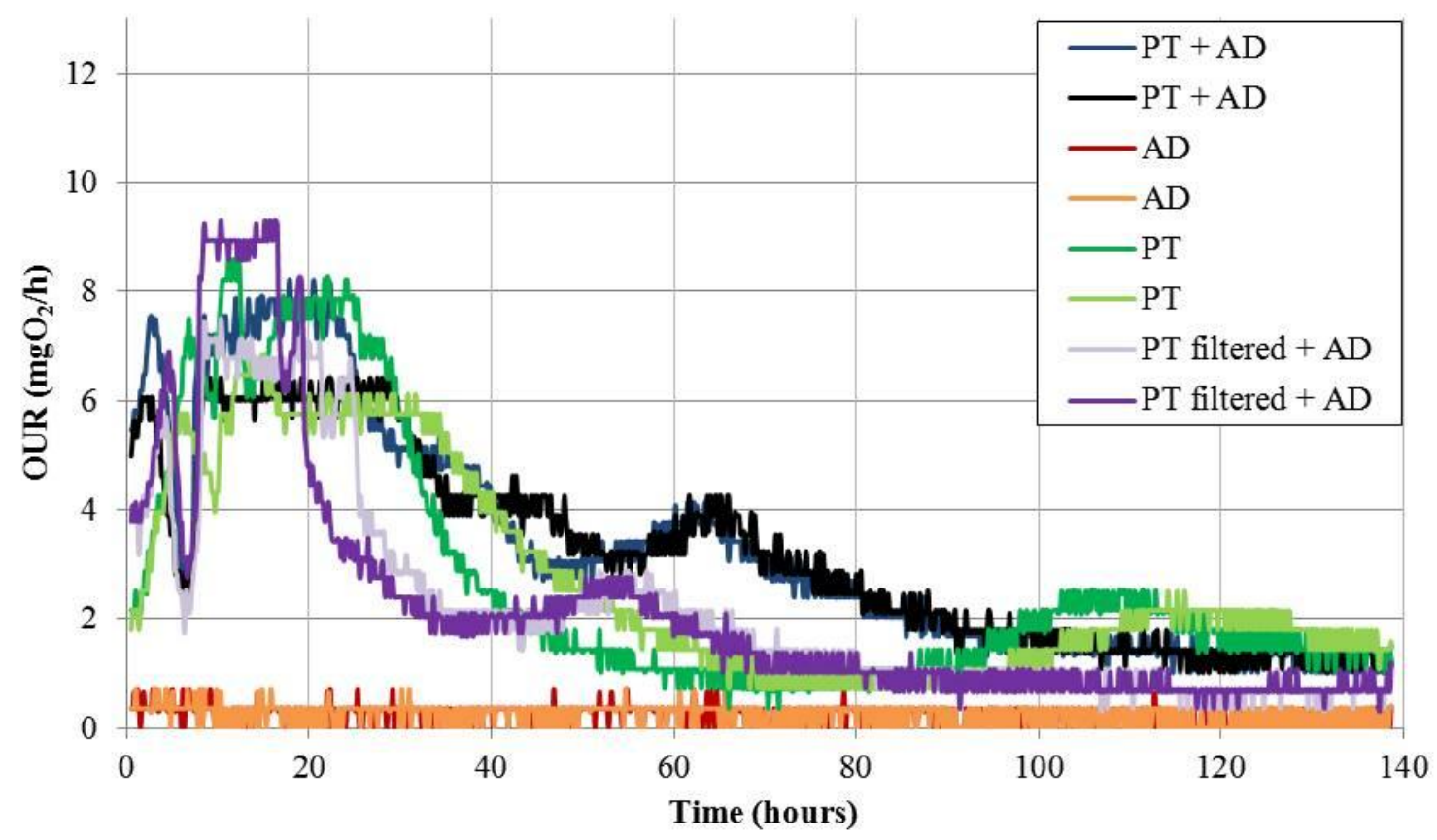

Figure C.8 Pretreated BR WAS and Acclimatized AD WAS - Test Started on Day 243 


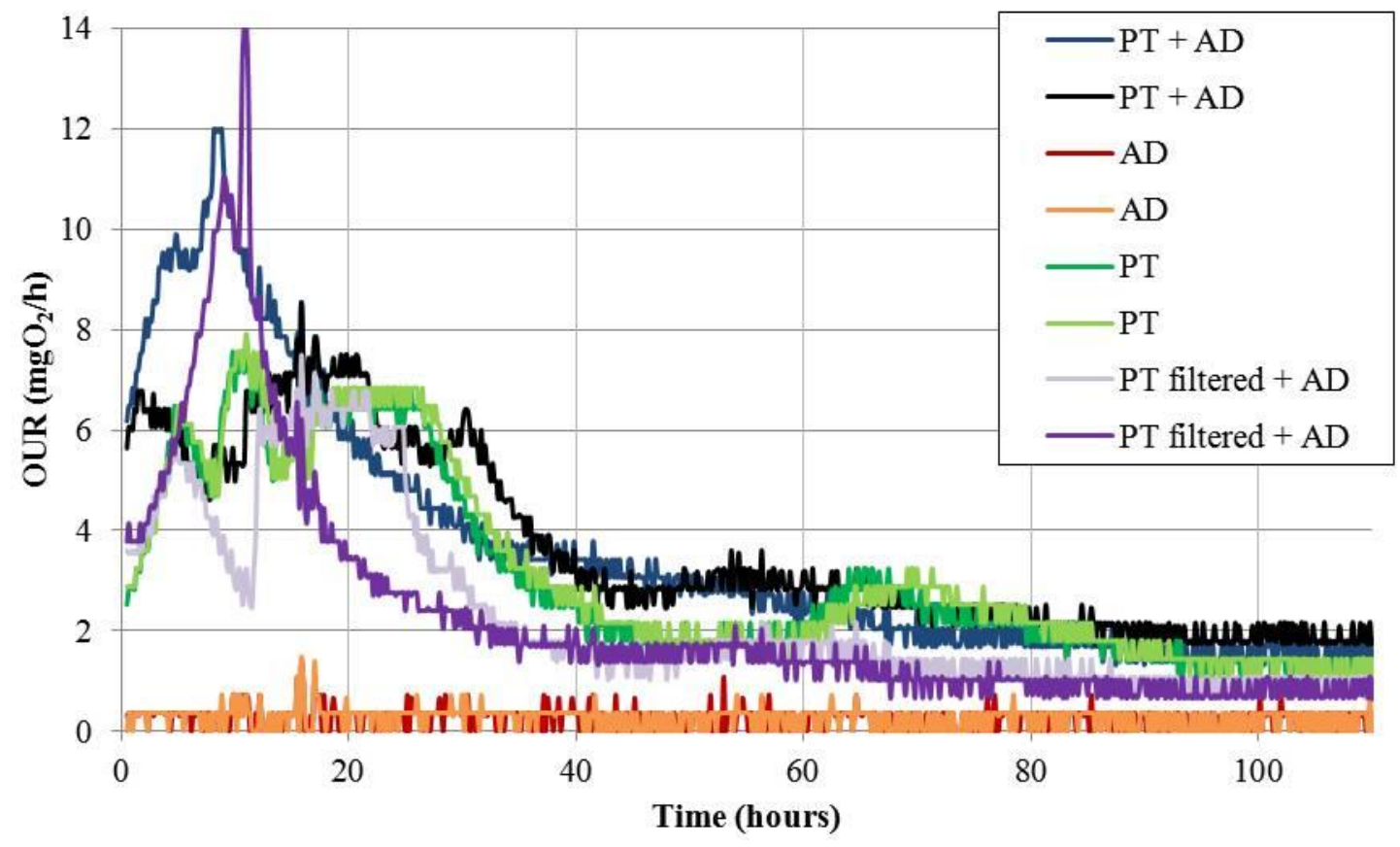

Figure C.9 Pretreated BR WAS and Acclimatized AD WAS - Test Started on Day 250

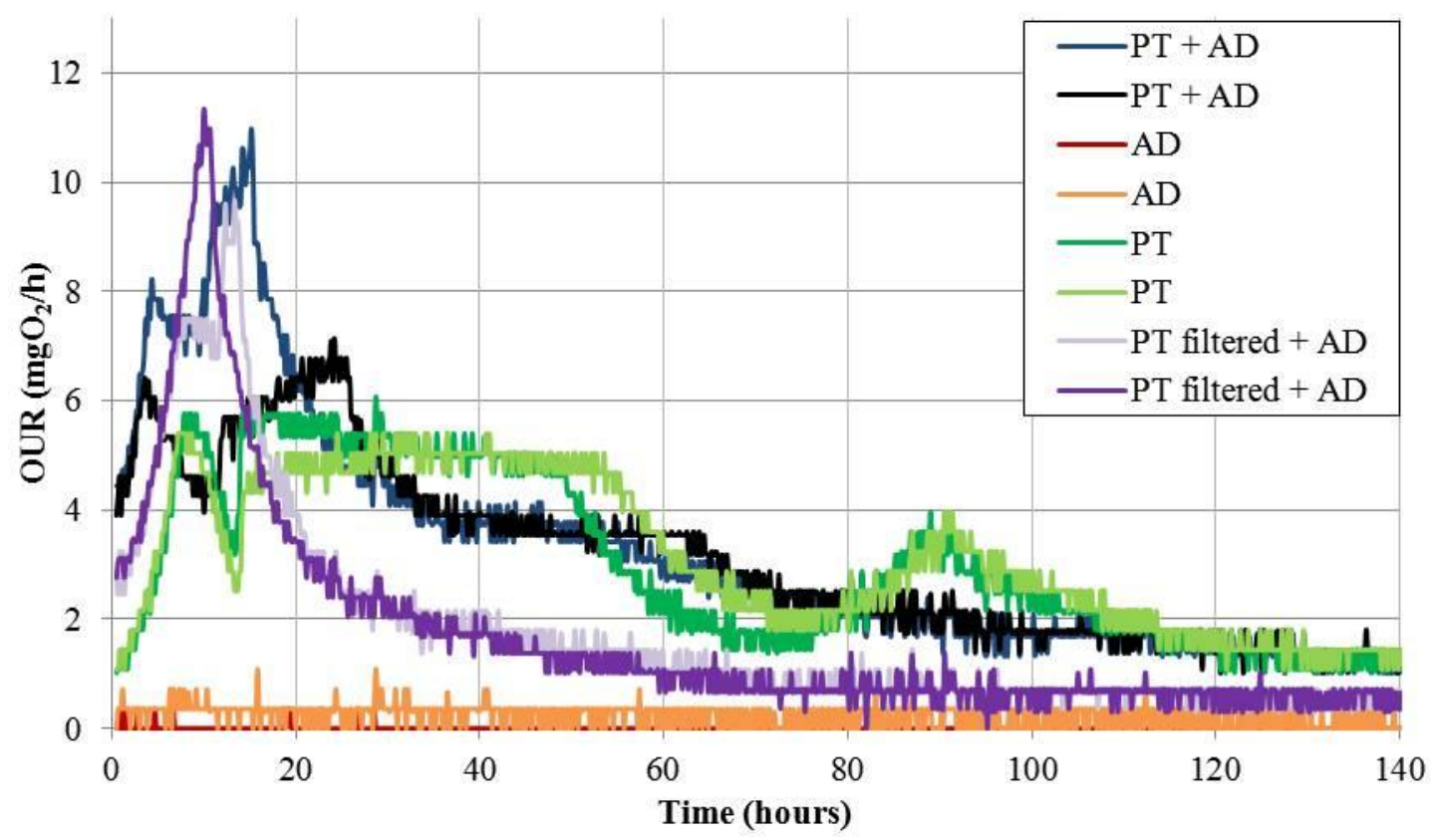

Figure C.10 Pretreated BR WAS and Acclimatized AD WAS - Test Started on Day 271 